ENGINEERING CHANGE NOTICE
1. EcN 658319

Proj.

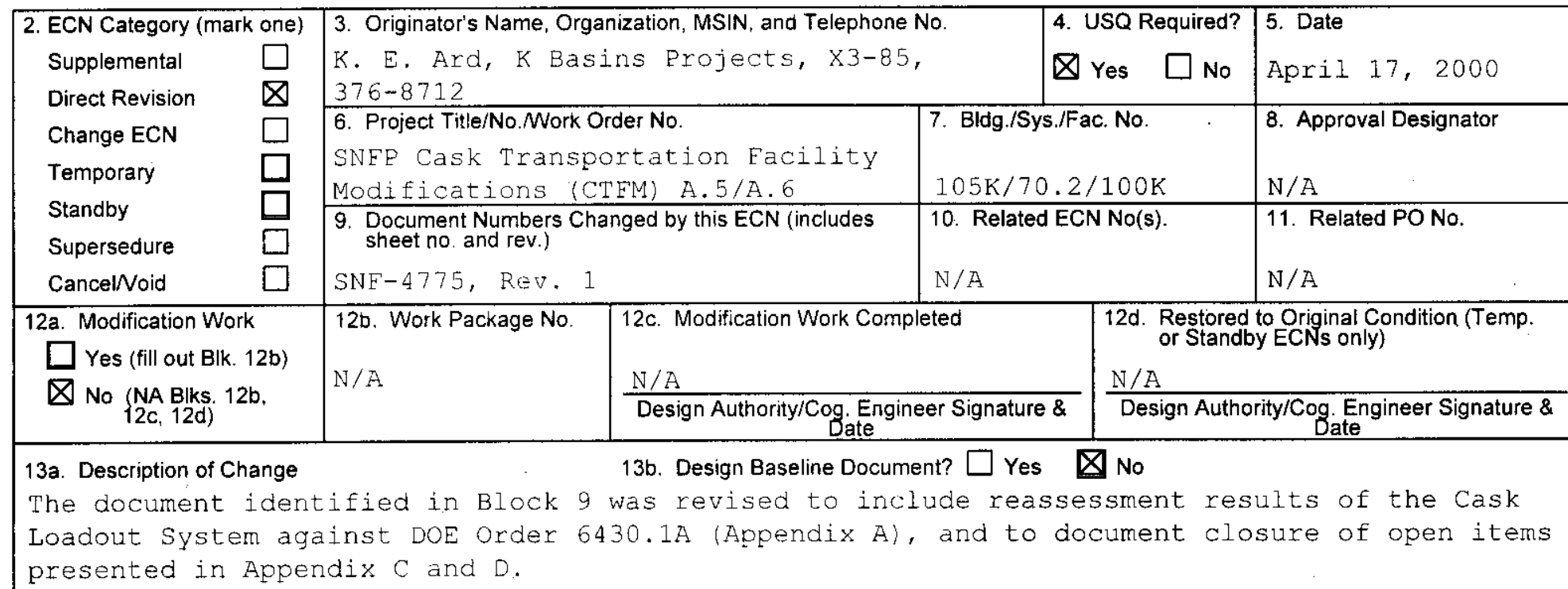

use: $K-00-0540$

\begin{tabular}{ll}
\hline 14a. Justification (mark one) \\
Criteria Change \\
Design Improvement \\
Environmental \\
Facility Deactivation \\
As-Found \\
Facilitate Const. \\
Const. Error/Omission \\
Design Error/Omission \\
$\square$
\end{tabular}

15. Distribution (include name, MSIN, and no. of copies)

K. E. Ard X3-85

T. B. Bergman R3-II

P. L. Lingle $\$ 2-51$

J. L. Weamer $\times 3-85$

K Basins Project Eiles $\times 3-85$

14b. Justification Details

Updated to document compliance with DOE Order 6430.1A.

Page 1 of 2 ECN 


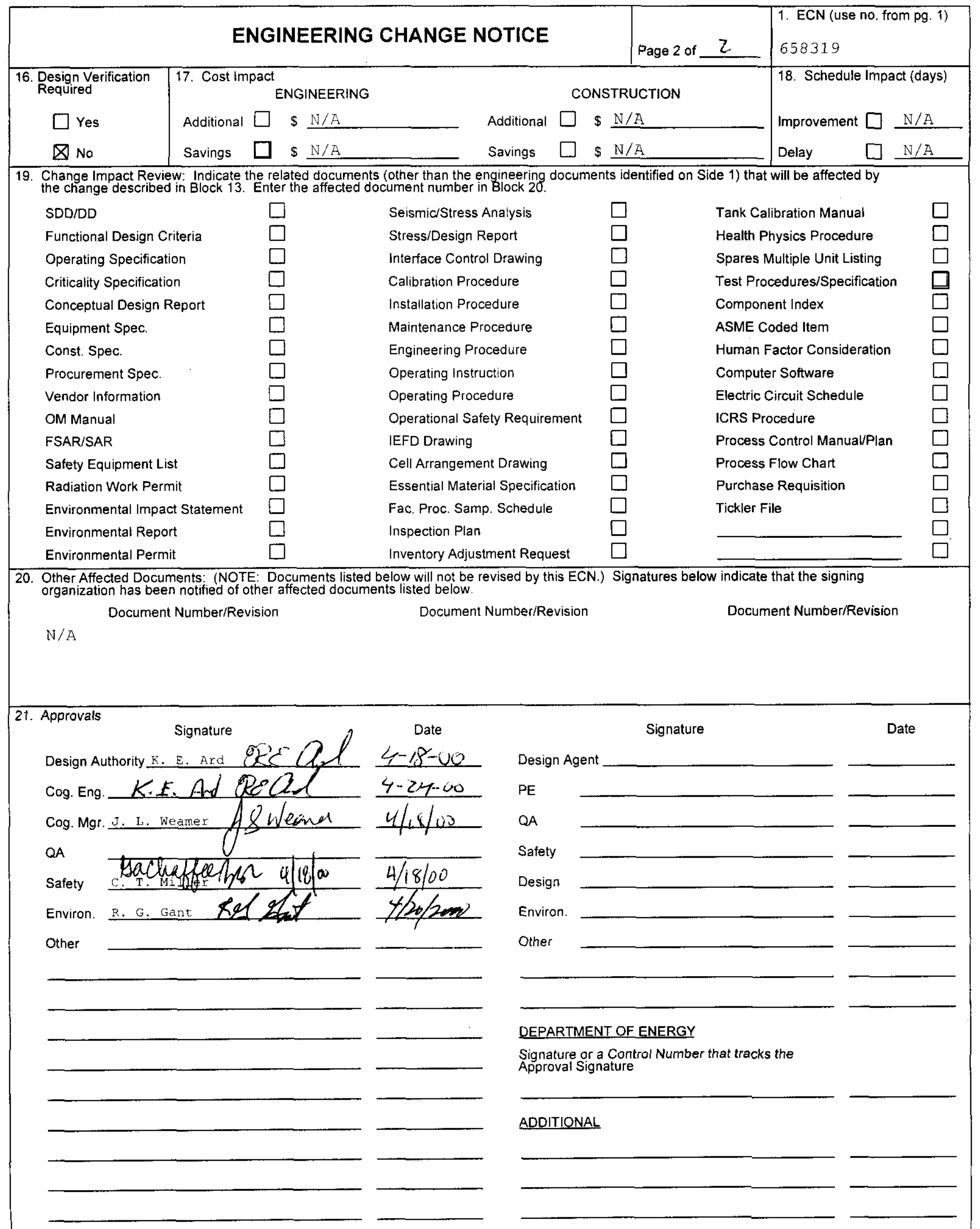


SNF-4775

Revision 2

\section{Evaluation of the Cask Transportation Facility Modifications (CTFM) Compliance to DOE Order 6430.1A - Project A.5/A.6}

Prepared for the U.S. Department of Energy

Assistant Secretary for Environmental Management

Project Hanford Management Contractor for the

U.S. Department of Energy under Contract DE-AC06-96RL13200

\section{Fluor Hanford}

P.0. Box 1000

Richland, Washington 
SNF-4775

Revision 2

ECN 658319

\title{
Evaluation of the Cask Transportation Facility Modifications (CTFM) Compliance to DOE Order 6430.1A - Project A.5/A.6
}

\author{
K. E. Ard
}

Fluor Hanford

Date Published

April 2000

Prepared for the U.S. Department of Energy

Assistant Secretary for Environmental Management

Project Hanford Management Contractor for the

U.S. Department of Energy under Contract DE-AC06-96RL13200

\section{Fluor Hanford}

P.O. Box 1000

Richland, Washington
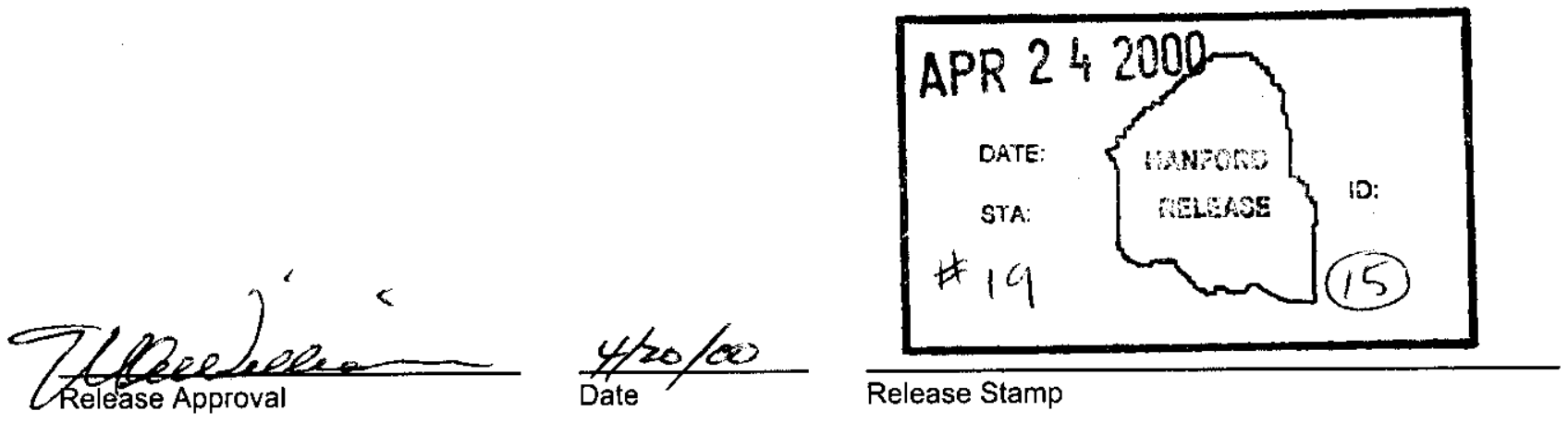
TRADEMARK DISCLAIMER

Reference herein to any specific commercial product, process, or service by trade name, trademark, manufacturer, or otherwise, does not necessarily constitute or imply its endorsement, recommendation, or favoring by the United States Government or any agency thereof or its contractors or subcontractors.

This report has been reproduced from the best available copy. Available in paper copy and microfiche.

Available electronically at http://www.doe.gov/bridge. Available for a processing fee to the U.S. Department of Energy and its contractors, in paper, from:

U.S. Department of Energy

Office of Scientific and Technical Information

P.O. Box 62

Oak Ridge, TN 37831-0062

Phone: 865-576-8401

Fax: $865-576-5728$

Email: reports@adonis.Osti.gov(423) 576-8401

Available for sail to the public, in paper, from:

U.S. Department of Commerce

National Technical Information Service

5285 Port Royal Road

Springfield, VA 22161

Phone: $800-553-6847$

Fax: $703-605-6900$

Email: orders@ntis.fedworld.gov

Online ordering:

http://www.ntis.gov/ordering.htm

Printed in the United States of America

Total Pages:

172 


\section{RECORD OF REVISION}

(2) Title

Evaluation of the Cask Transportation Facility Modification (CTFM) Compliance to DOE Order 6430.1A Project A.5/A.6

Change Control Record

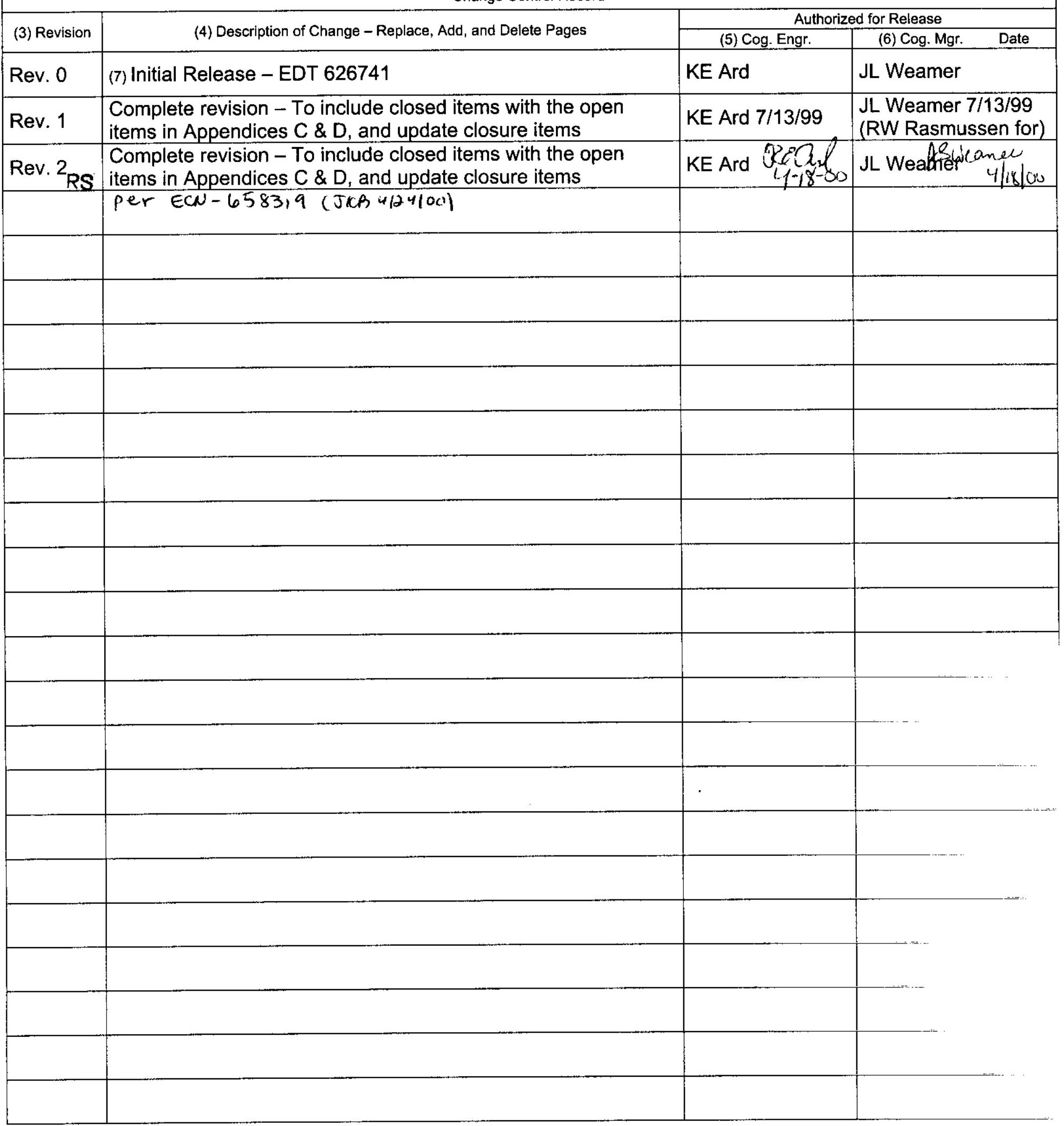




\section{TABLE OF CONTENTS}

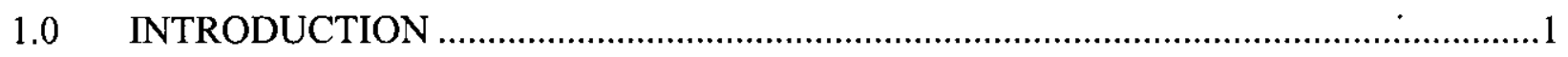

2.0 DOE ORDER 6430.1A - COMPLIANCE ASSESSMENT .............................................

3.0 DOE ORDER 6430.1A - OPEN ITEMS .................................................................2

INDEX FOR REFERENCED TRADEMARKS ...........................................................

APPENDIX A Cask Loadout System - General Design Criteria ....................................... A-1

APPENDIX B Cranes/Other Modifications - General Design Criteria.................................

APPENDIX C Cask Loadout System (CLS) Table - General Design Criteria, Open Items

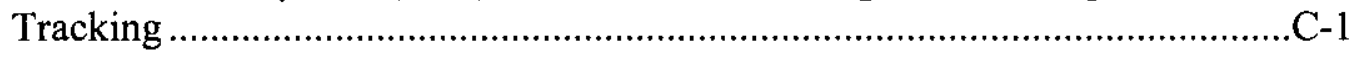

APPENDIX D Cranes - General Design Criteria, Open Items Tracking

Other Mods - General Design Criteria, Open Items Tracking ...................... D-1

Charge Code: $105463 / \mathrm{B} 000$

B\&R Code: $\quad$ EW04J1110

Key Words: K Basins, Spent Nuclear Fuel (SNF), Cask Transportation Facility Modifications (CTFM), Project A.5/A.6, Compliance, 6430.1A, 100K, Cask Loadout Systems (CLS).

Abstract: This report was prepared to evaluate the compliance of CTFM to DOE Order 6430.1A. 


\section{Evaluation of the Cask Transportation Facility Modifications (CTFM) Compliance to DOE Order 6430.1A}

\section{$1.0 \quad$ INTRODUCTION}

This document presents the results of an evaluation that was performed to assess compliance of the $\mathrm{K}$ West (KW) Cask Transportation Facility Modifications (CTFM) designs against applicable requirements of Department of Energy (DOE) Order 6430.1A, General Design Criteria. This evaluation was grouped under two categories described as Cask Loadout System (CLS) and Cranes/Other Modifications. The systems, structures, and components under each of these two categories are grouped in Table 1 below.

Table 1. Evaluation Categories and Related Work Scope.

\begin{tabular}{|l|l|}
\hline \multicolumn{1}{|c|}{ Cask Loadout System (CLS) } & \multicolumn{1}{c|}{ Crane/Other Modifications } \\
\hline \hline Multi-Canister Overpack Loading System (MLS) & Transfer Bay Crane \\
\hline Immersion Pail Support Structure & Windbreak Structure \\
\hline Immersion Pail & Compressed Air System \\
\hline Alignment Hardware & $\begin{array}{l}\text { Equipment Lay Down and Decontamination } \\
\text { Area }\end{array}$ \\
\hline & $\begin{array}{l}\text { General Area Preparations (e.g., demolition, } \\
\text { conduit and piping reroutes) }\end{array}$ \\
\hline & Corridor 7 Door and Window \\
\hline & Operator Interface Platform \\
\hline
\end{tabular}

With exception to the CLS, the original compliance assessment was not updated to preserve the results of the original assessment. Safety Evaluation Report (SER) conditions of approval in the U.S. Department of Energy, Richland Operations Office (RL) letter 99-SFD-082, dated 2/23/99, included reassessment of the CLS against 6430.1A to address the CLS alternative approach described in Fluor Hanford, Inc. (FH) letter FDH-9955223, dated July 29, 1999 and approved in RL letter 99-SFD-160, dated 8/2/99. The reassessment was transmitted to RL in FH letter FDH9956616, dated 10/25/99. At the time that the $6430.1 \mathrm{~A}$ reassessment was performed, some open items were closed. The new CLS assessment is provided in this report, however; open items from the original CLS assessment have been tracked, closed, and documented in this report.

\subsection{DOE ORDER 6430.1A - COMPLIANCE ASSESSMENT}

Appendices A and B provide the results of the compliance assessment performed for the Cask Loadout System and Cranes/Other Modifications, respectively. Assessment results for Cranes and Other Modifications are on pages B-2 through B-21 and B-21 through B-99, respectively. The results are presented in a tabular format, which includes appropriate DOE Order 6430.1A section number, criteria description, and evaluation results. 
SNF-4775, Rev. 2

Project A.5/A.6

\subsection{DOE ORDER 6430.1A - OPEN ITEMS}

Appendices $\mathrm{C}$ and $\mathrm{D}$ provide the final closure action and closure date of items that were identified as open issues during the original assessment. All open items have been closed. Final status for Cranes and Other Modifications are on pages D-1 through D-5 and D-6 through D-12, respectively. The status is provided in a tabular format, which includes appropriate

DOE Order 6430.1A section number, criteria description, evaluation results, assignee, due date, closure action and date. 


\section{INDEX FOR REFERENCED TRADEMARKS}

\begin{tabular}{|c|c|}
\hline Word Mark: & $A N S I$ \\
\hline Owner Name: & (Registrant) American National Standards Institute, Incorporated \\
\hline Type of Mark: & Trademark \\
\hline Word Mark: & FM \\
\hline Owner Name: & (Registrant) Factory Mutual Engineering Corporation \\
\hline Type of Mark: & Certification Mark \\
\hline Word Mark: & IEEE \\
\hline Owner Name: & (Registrant) Institute of Electrical and Electronics Engineers, Inc., The \\
\hline Type of Mark: & Service Mark \\
\hline Work Mark: & $N F P A$ \\
\hline Owner Name: & (Registrant) National Fire Protection Association, Inc. \\
\hline Type of Mark: & Trademark \\
\hline Word Mark: & $U L$ \\
\hline Owner Name: & (Registrant) Underwriters Laboratories, Inc. \\
\hline Type of Mark: & Certification Mark \\
\hline
\end{tabular}


SNF-4775, Rev. 2

Project A.5/A.6

\section{APPENDIX A}

\section{CASK LOADOUT SYSTEM (CLS)}

\section{DOE ORDER 6430.1A - GENERAL DESIGN CRITERIA}

\section{CONSISTING OF 37 PAGES} INCLUDING COVERSHEET

Page A-1 
SNF-4775, Rev. 2

Project A.5/A.6

APPENDIX A

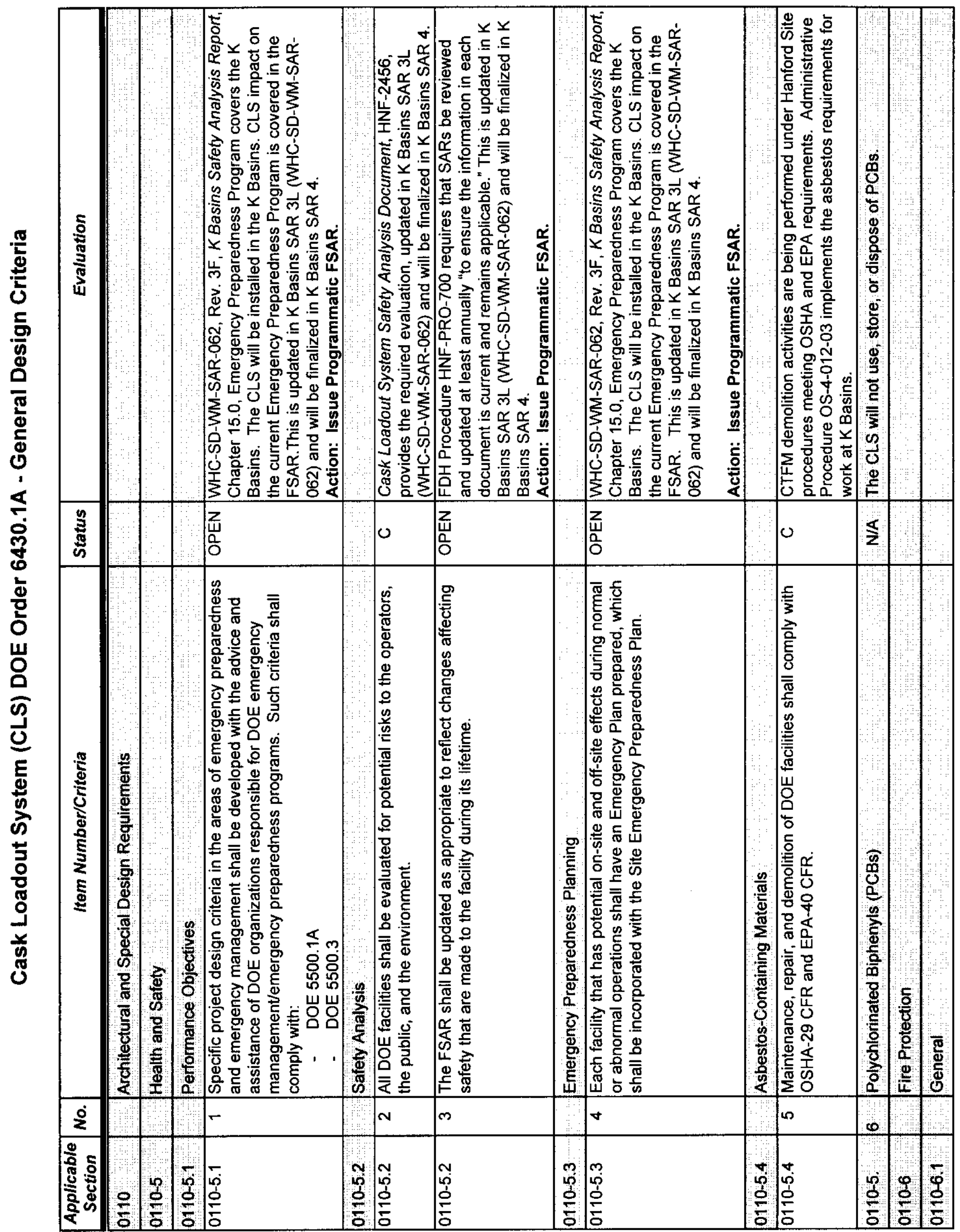

Page A-2 
SNF-4775, Rev. 2

Project A.5/A.6

APPENDIX A

\begin{tabular}{|c|c|c|c|c|c|c|c|c|c|}
\hline 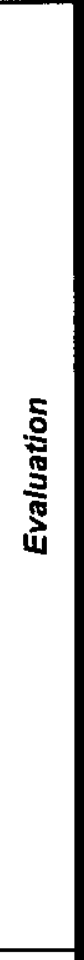 & 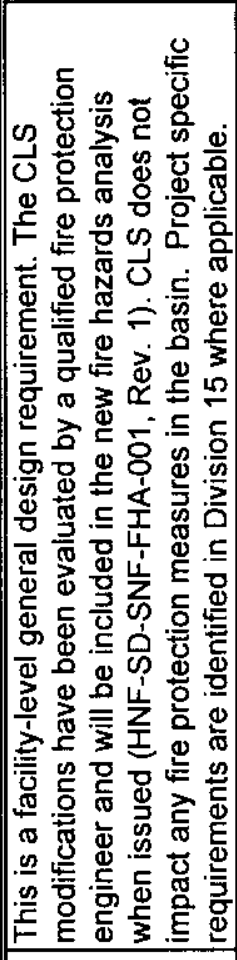 & 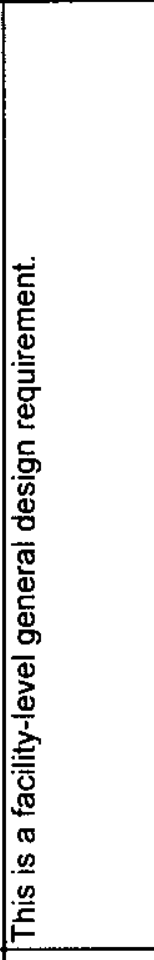 & 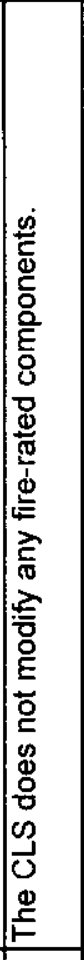 & 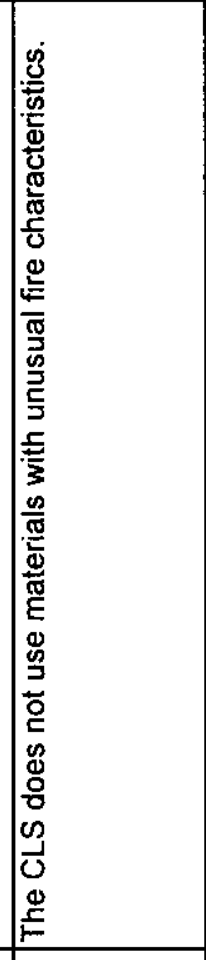 & 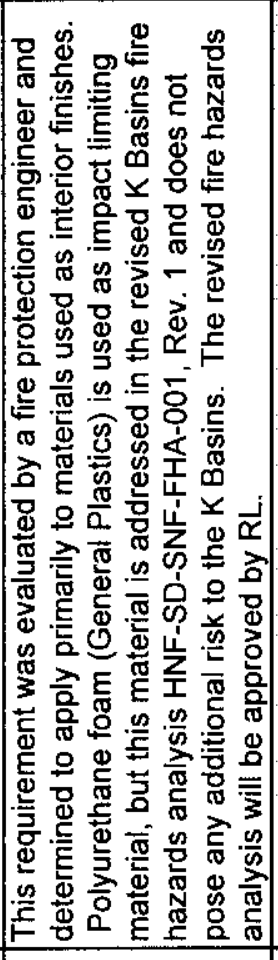 & & 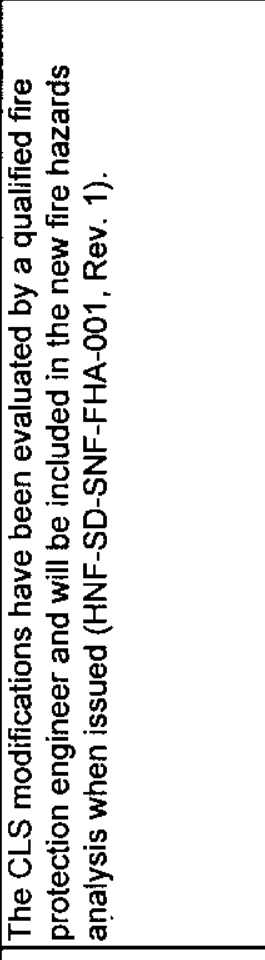 & 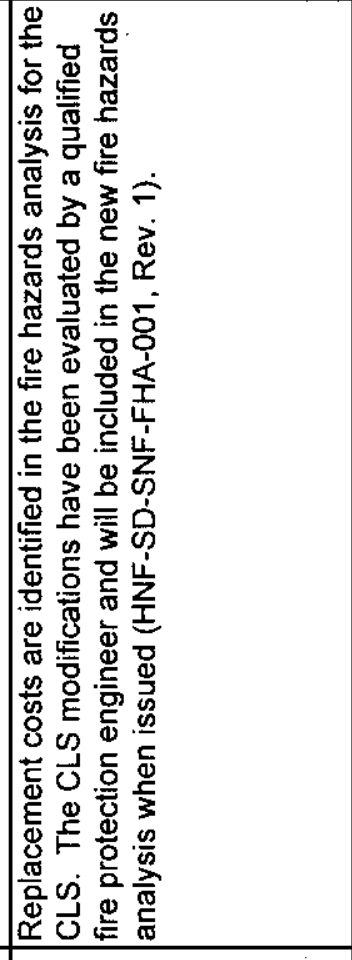 & \\
\hline 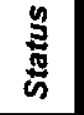 & 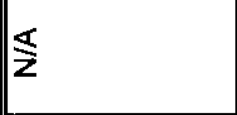 & $\frac{\leqslant}{z}$ & $\mid \widehat{\underline{z}}$ & $\frac{\$}{\mathbf{z}}$ & $\mid \S$ & & 0 & 0 & \\
\hline 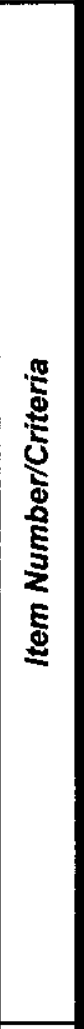 & 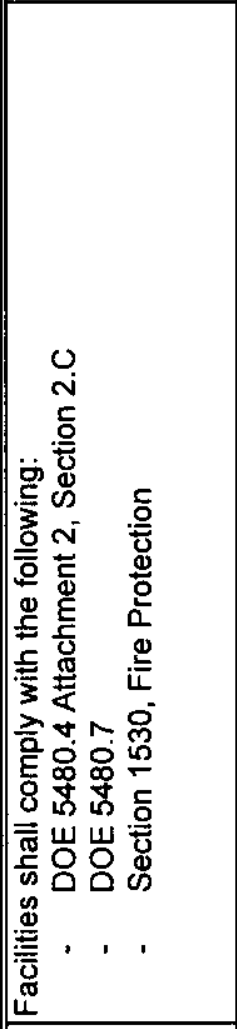 & 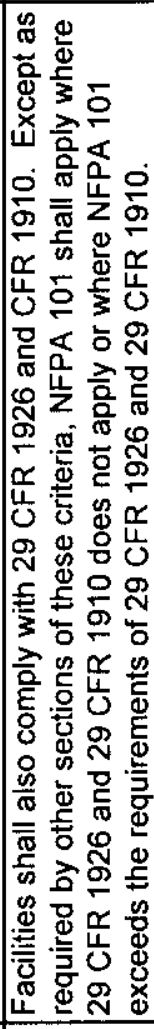 & 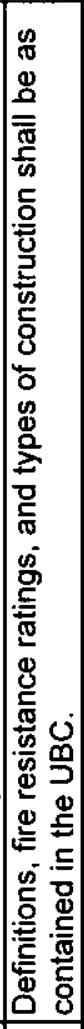 & 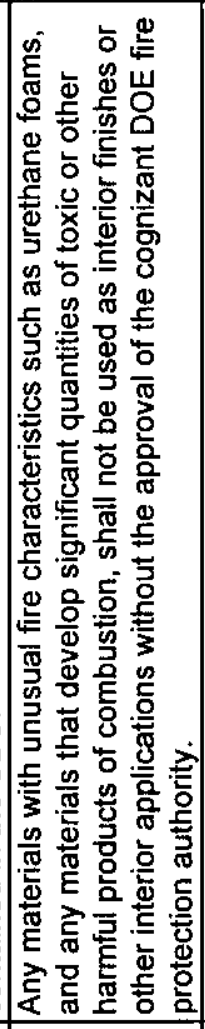 & 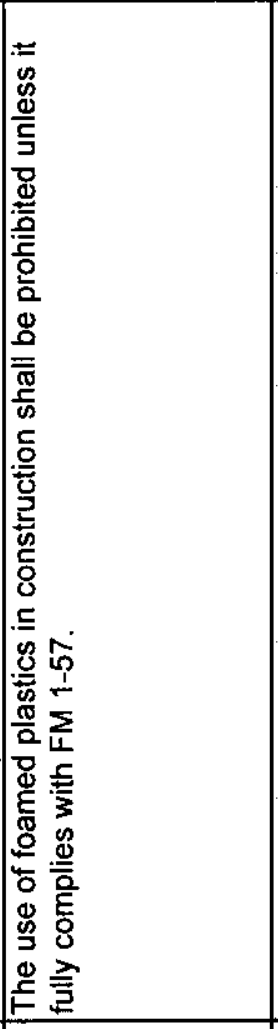 & 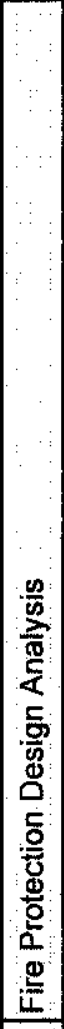 & 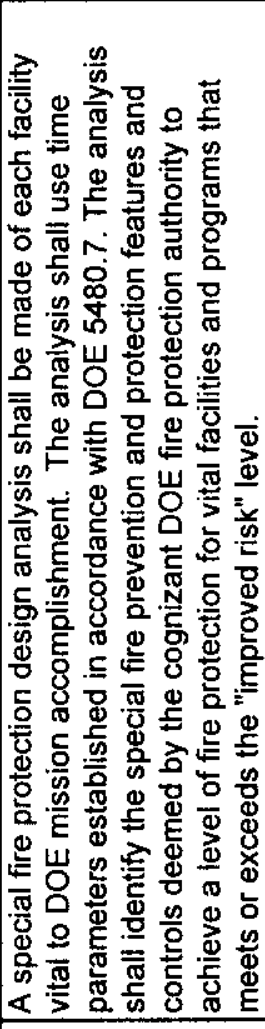 & 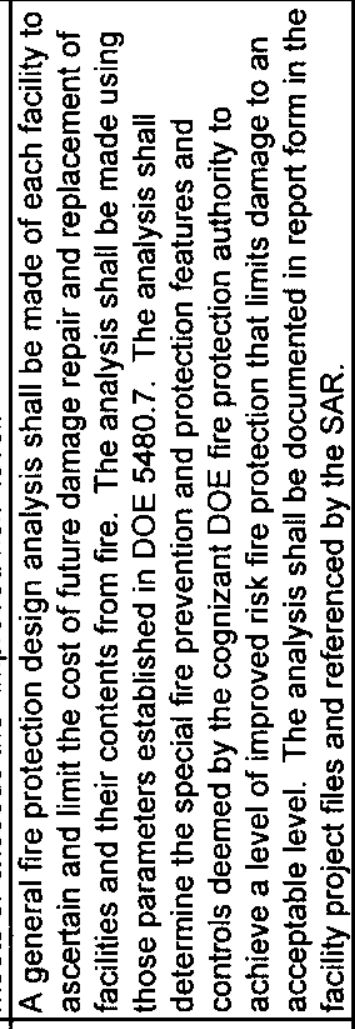 & o \\
\hline$\stackrel{0}{2}$ & ${ }^{\wedge}$ & $\infty$ & $\sigma$ & 은 & $\mp$ & & $\cong$ & $m$ & 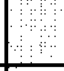 \\
\hline 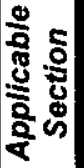 & $\mid \begin{array}{c}\bar{c} \\
0 \\
0 \\
0\end{array}$ & 产 & & $\mid \begin{array}{l}\varphi \\
0 \\
0 \\
0 \\
0\end{array}$ & $\frac{0}{0}$ & & $\begin{array}{l}\underline{\varphi} \\
\vdots \\
\vdots \\
\\
\end{array}$ & $\frac{0}{0}$ & $\frac{9}{6}$ \\
\hline
\end{tabular}


SNF-4775, Rev. 2

Project A.5/A.6

APPENDIX A

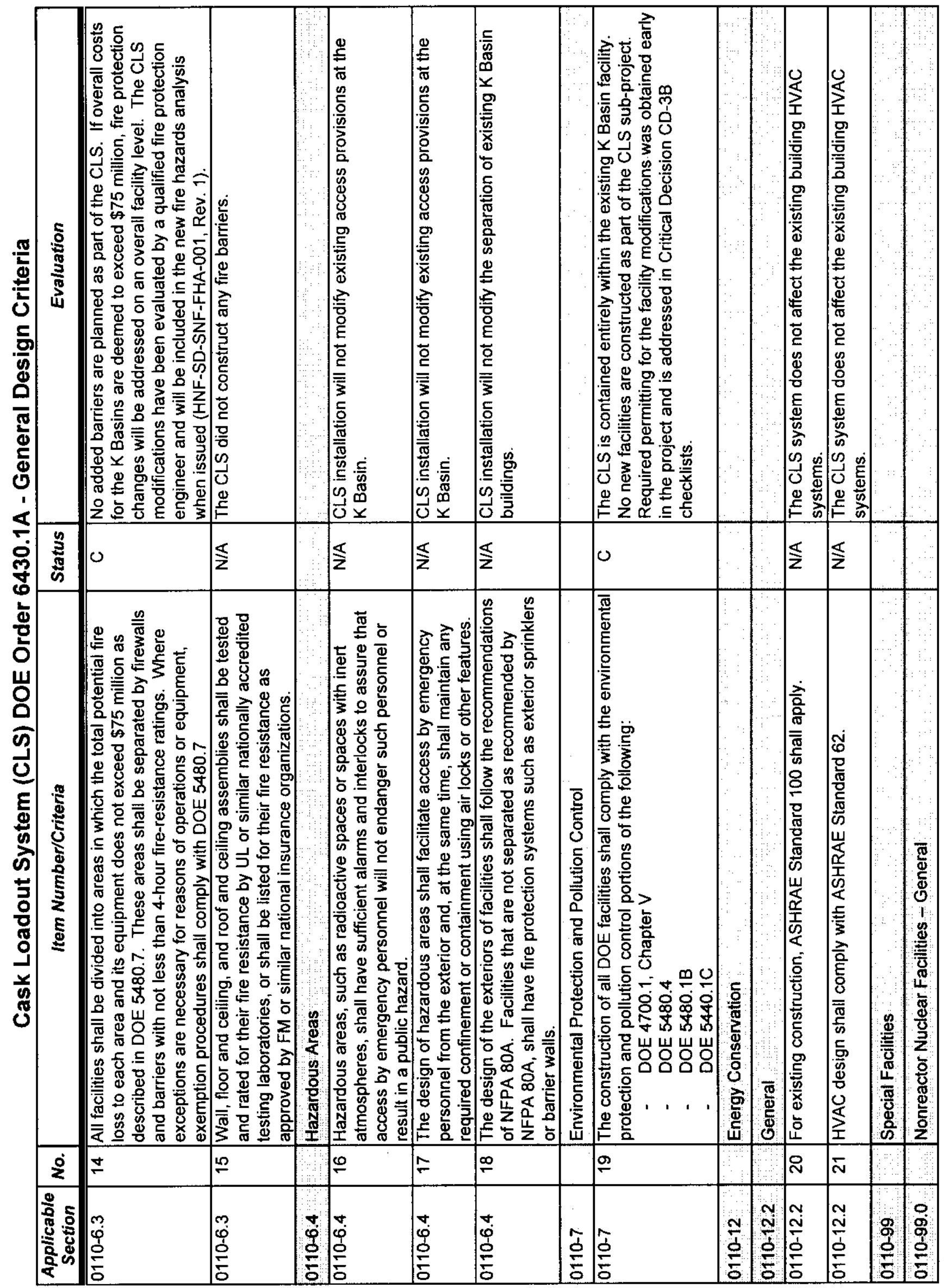


SNF-4775, Rev. 2

Project A.5/A.6

APPENDIX A

\begin{tabular}{|c|c|c|c|c|c|c|c|c|c|c|c|}
\hline 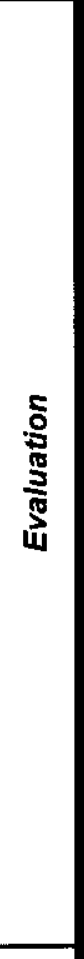 & 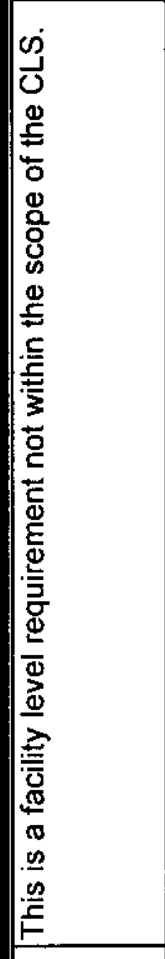 & 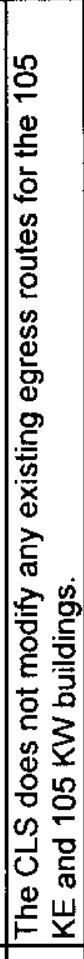 & 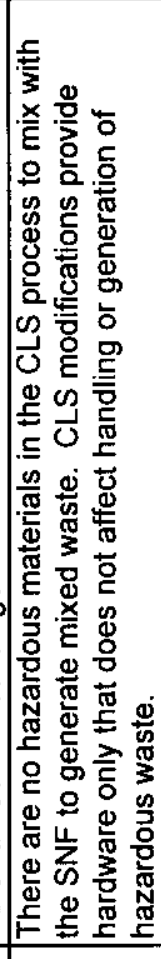 & 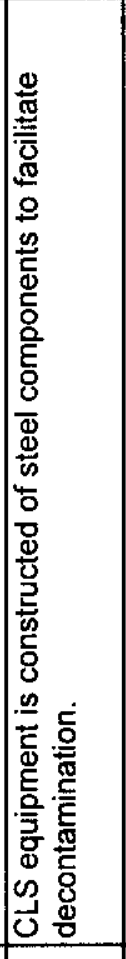 & & 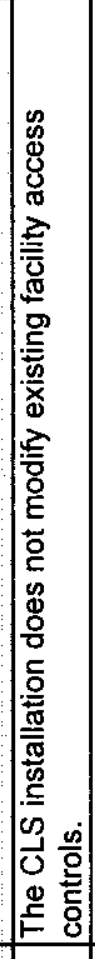 & 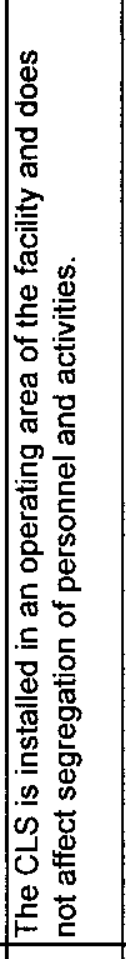 & 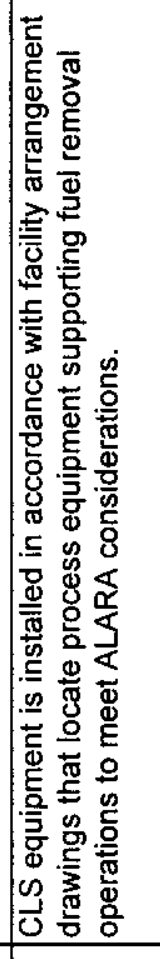 & 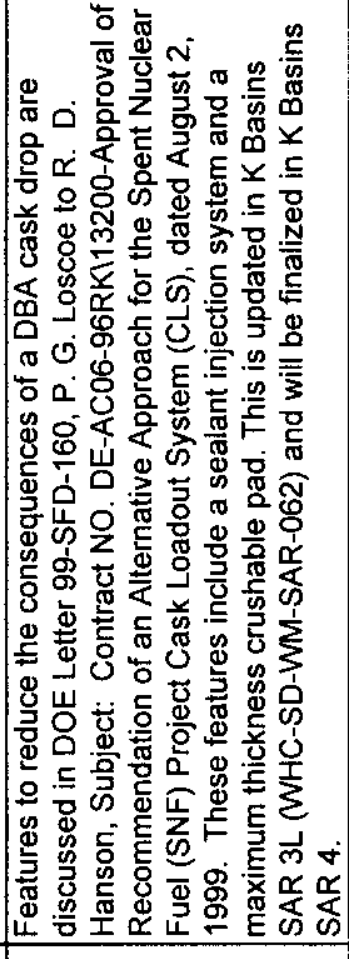 & 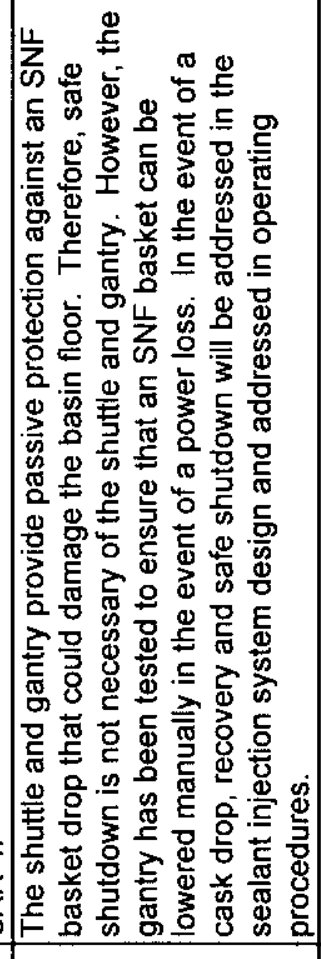 & 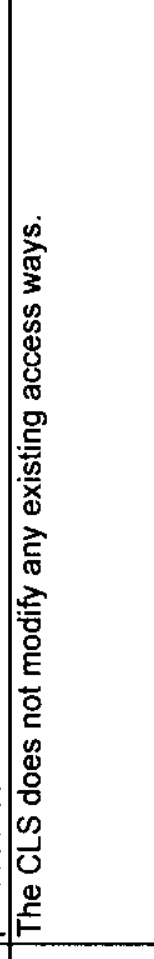 \\
\hline 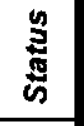 & $\stackrel{\S}{\mathbf{z}}$ & 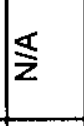 & 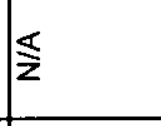 & 0 & & 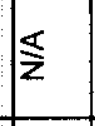 & $\stackrel{\leqslant}{\mathbf{z}}$ & 0 & 0 & 0 & 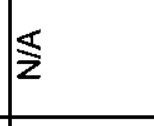 \\
\hline 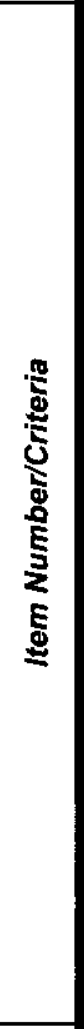 & 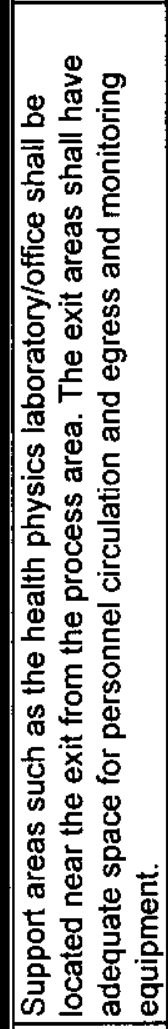 & 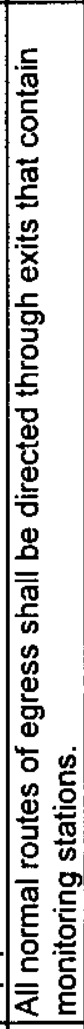 & 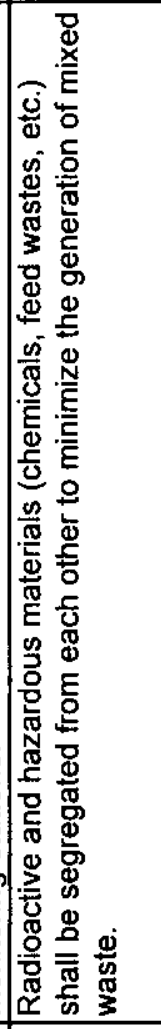 & 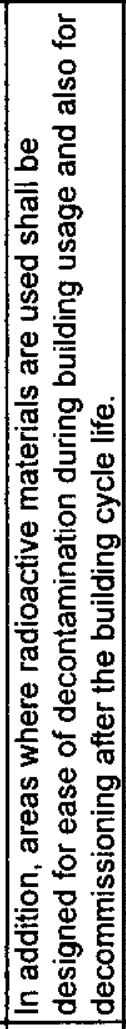 & 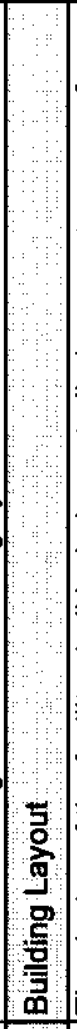 & 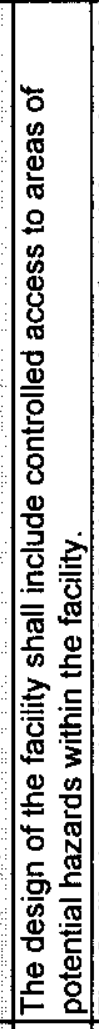 & 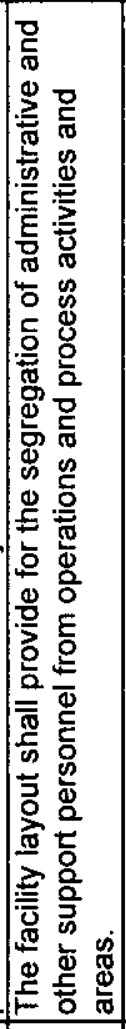 & 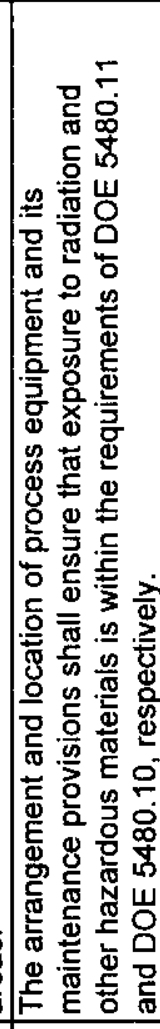 & 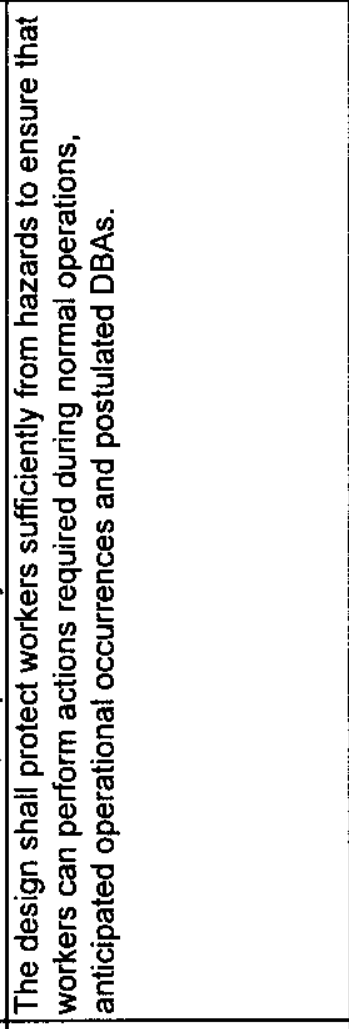 & 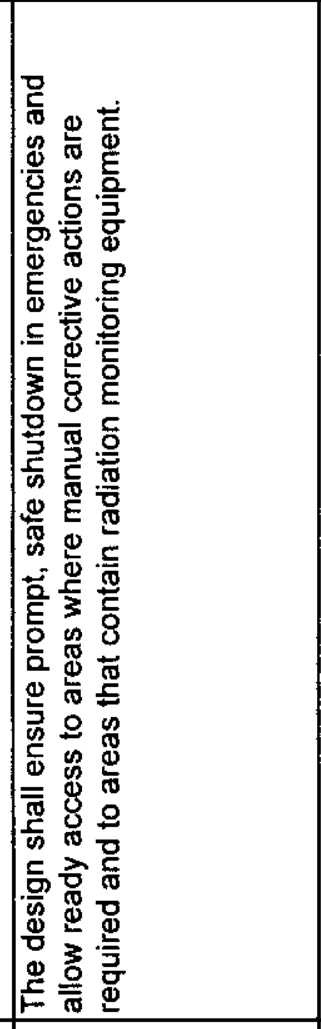 & 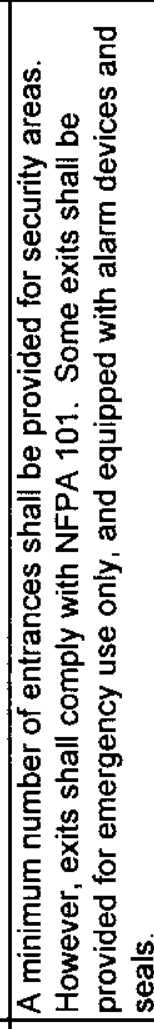 \\
\hline z & $\mathbb{N}$ & $\stackrel{\sim}{N}$ & ম & జ & & $\mathscr{~}$ & $\sqrt{N}$ & ని & ন্ & 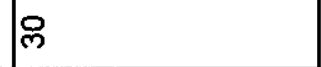 & $\bar{m}$ \\
\hline 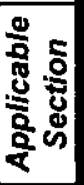 & $\frac{0}{0}$ & 离 & 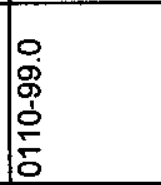 & o & $\begin{array}{c} \\
0 \\
0 \\
0 \\
\\
\end{array}$ & 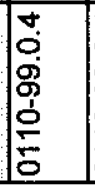 & 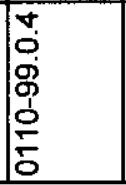 & 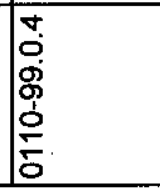 & 10 & 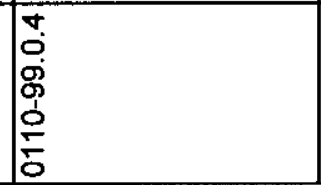 & 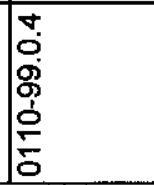 \\
\hline
\end{tabular}


SNF-4775, Rev. 2

Project A.5/A.6

APPENDIX A

\begin{tabular}{|c|c|c|c|c|c|c|c|c|c|c|c|c|c|}
\hline 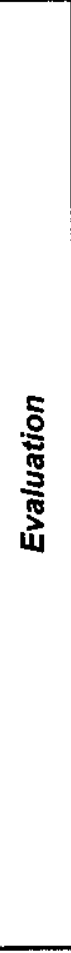 & 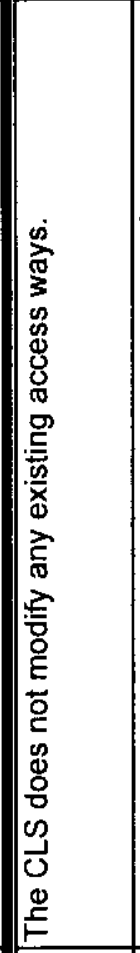 & 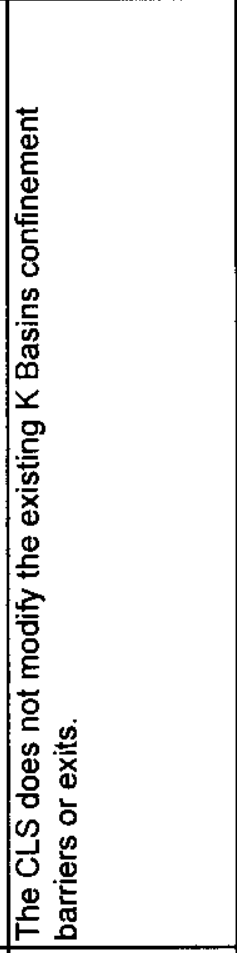 & 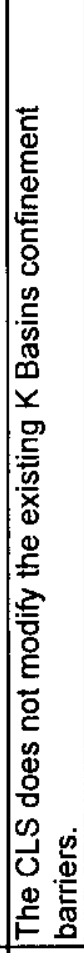 & 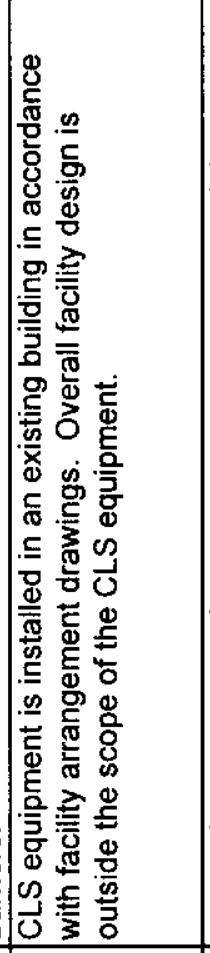 & 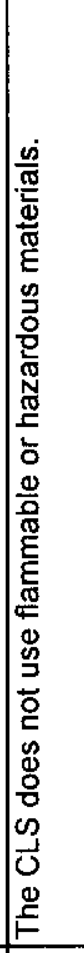 & 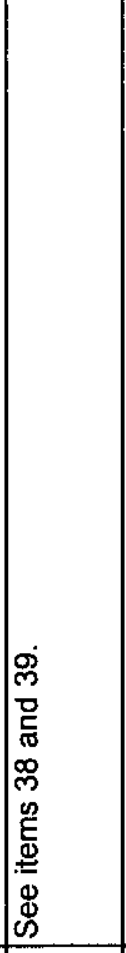 & 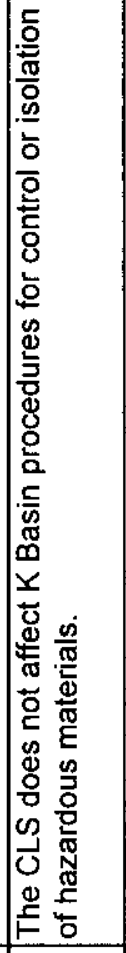 & 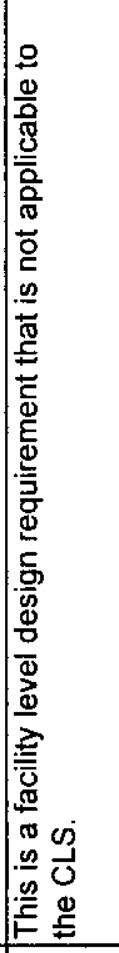 & 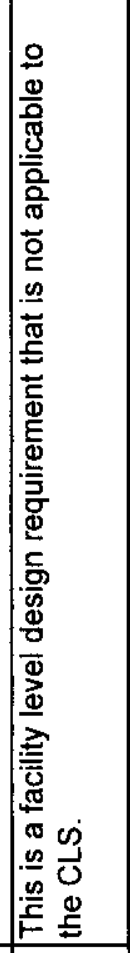 & 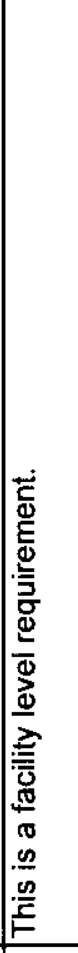 & 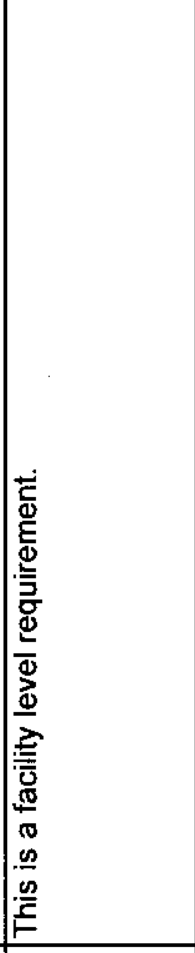 & 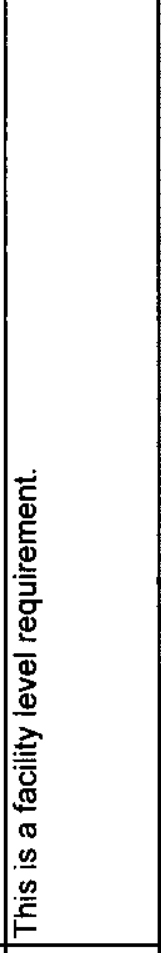 & \\
\hline 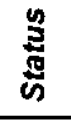 & $\| \lesssim$ & $\$$ & $\$ \frac{\xi}{\mathbf{z}}$ & $\stackrel{\S}{\mathbf{z}}$ & 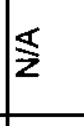 & 1 & $\mathbb{\mathbf { z }}$ & 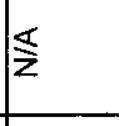 & 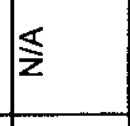 & $\stackrel{\leqslant}{\mathbf{z}}$ & $\frac{\varsigma}{z}$ & $\$$ & \\
\hline 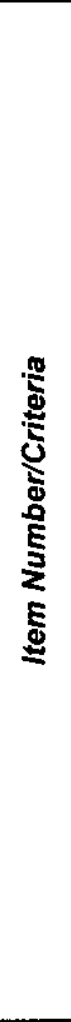 & 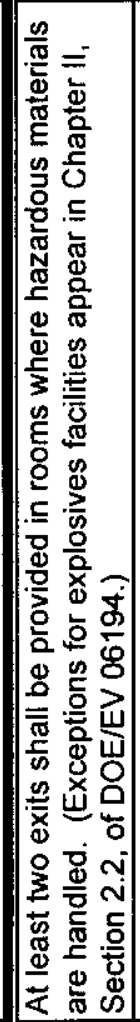 & 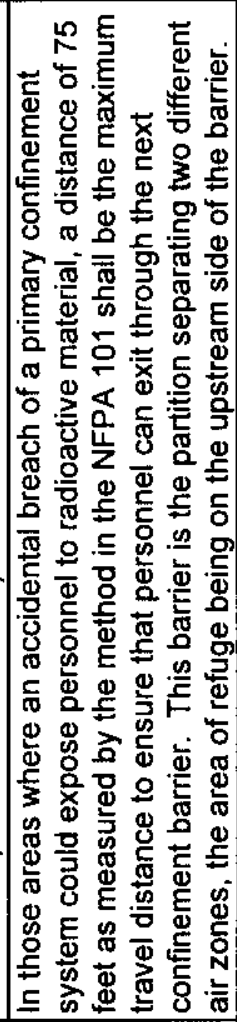 & 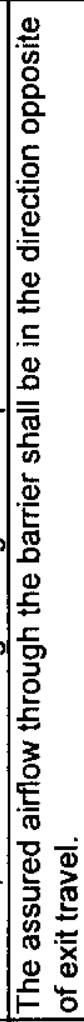 & 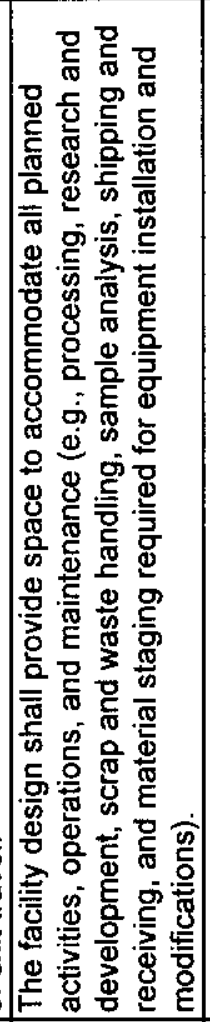 & 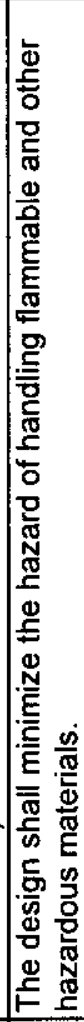 & 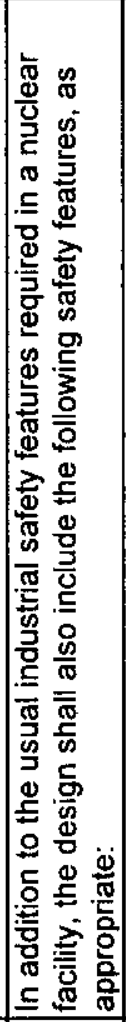 & 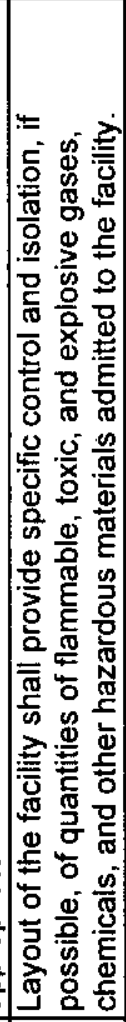 & 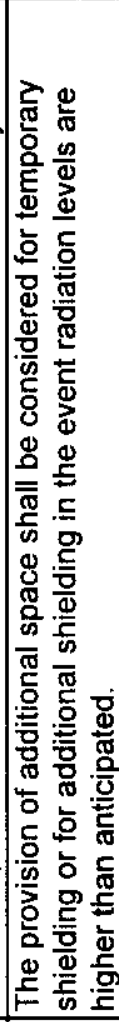 & 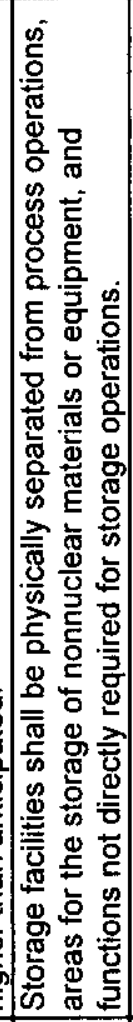 & 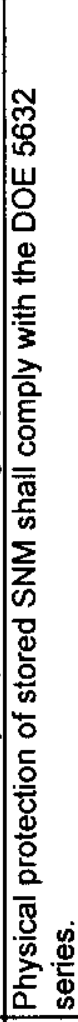 & 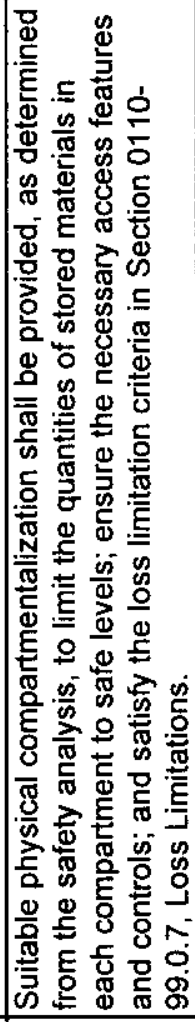 & 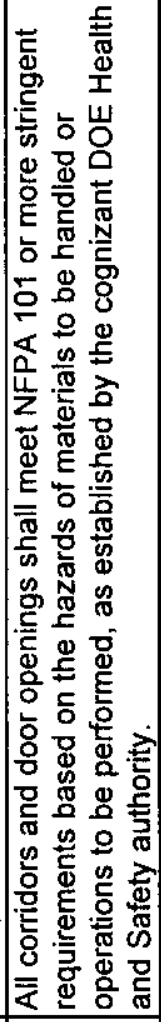 & 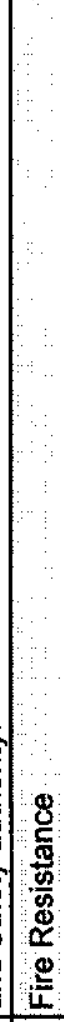 \\
\hline${ }_{2}^{\circ}$ & लू & m్ల & মু & $\stackrel{m}{ }$ & 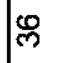 & $\hat{m}$ & ळ్ల & প্ল & of & $\bar{v}$ & พ & $\stackrel{?}{*}$ & \\
\hline 品 & 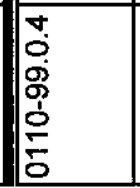 & 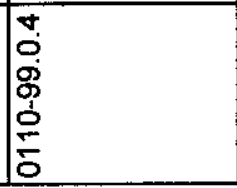 & 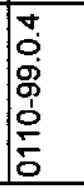 & 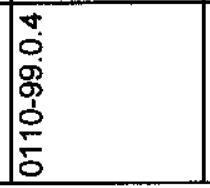 & 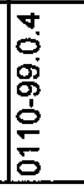 & 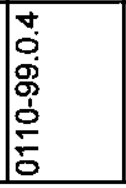 & 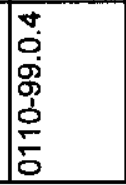 & 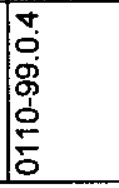 & 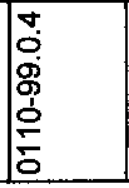 & 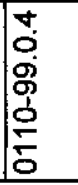 & 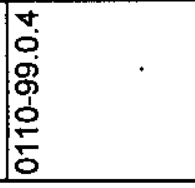 & 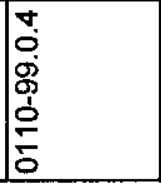 & $\begin{array}{l}0 \\
0 \\
8 \\
\delta \\
0 \\
\\
\end{array}$ \\
\hline
\end{tabular}


SNF-4775, Rev. 2

Project A.5/A.6

APPENDIX A

\begin{tabular}{|c|c|c|c|c|c|c|c|c|}
\hline 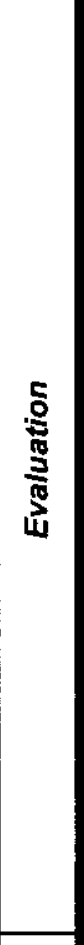 & 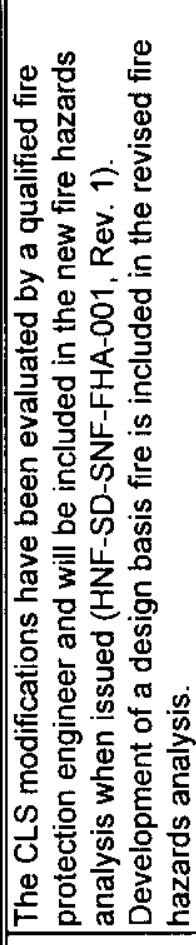 & 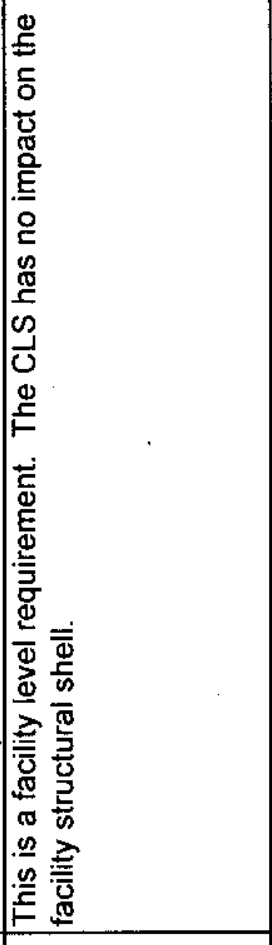 & 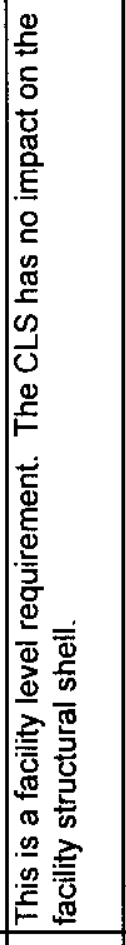 & 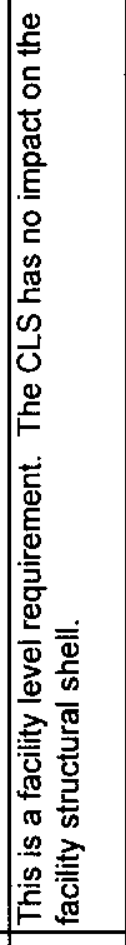 & & 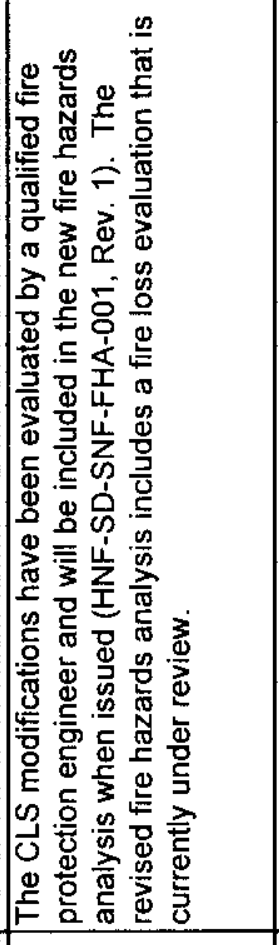 & & 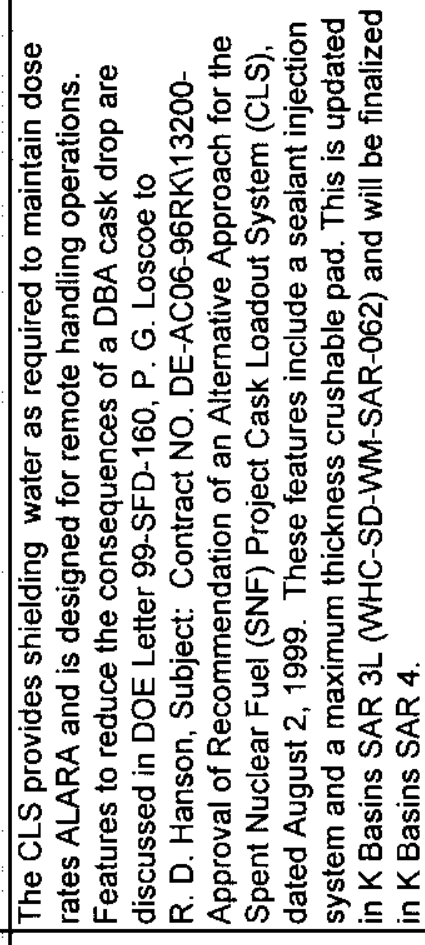 \\
\hline $\begin{array}{l}3 \\
\text { 劳 } \\
\text { s }\end{array}$ & 0 & $\mid \S$ & $\stackrel{\S}{\mathbf{z}}$ & $\mid \frac{\leqslant}{z}$ & & 0 & & 0 \\
\hline 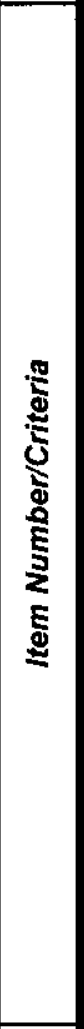 & 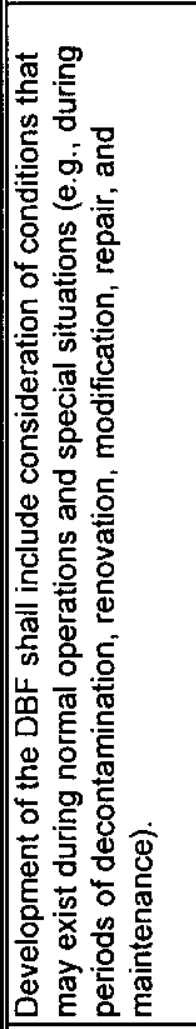 & 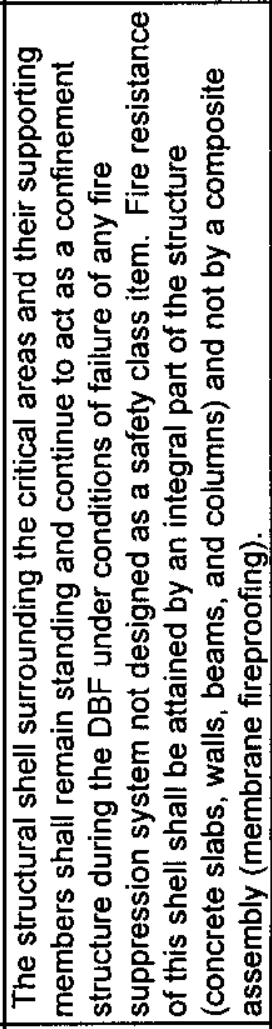 & 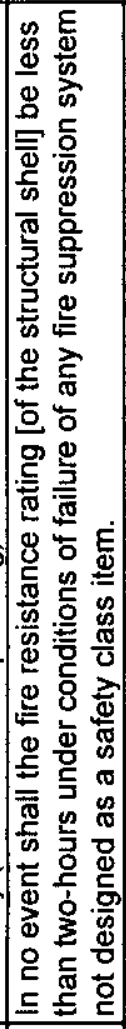 & 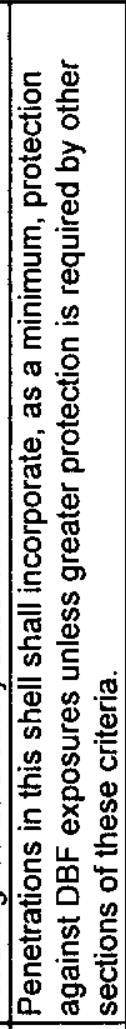 & 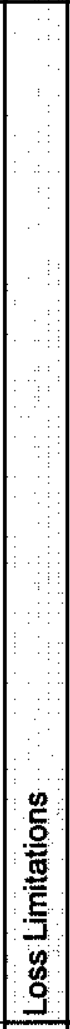 & 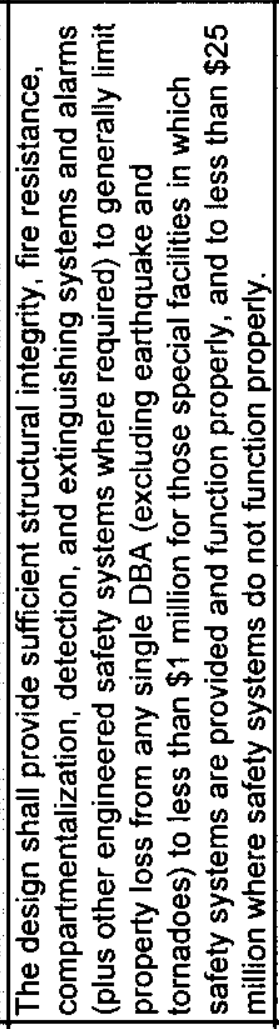 & 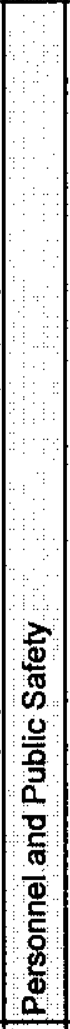 & 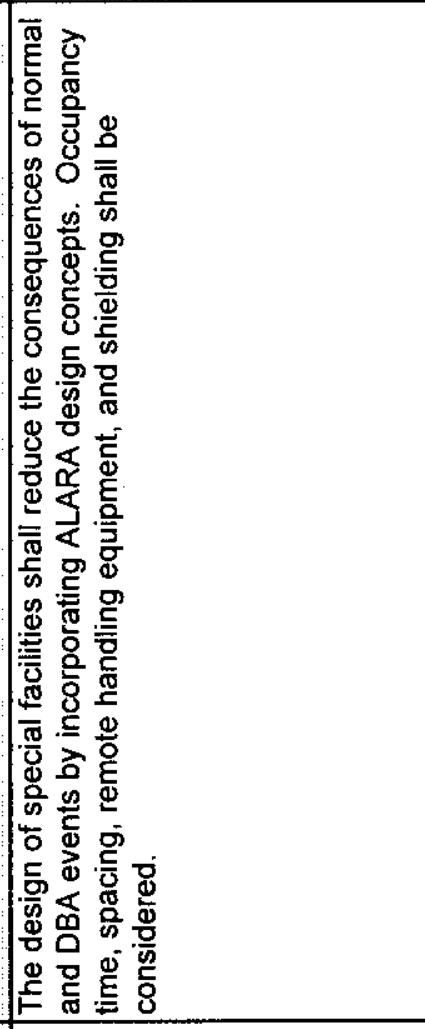 \\
\hline 之 & 寸 & 舟 & 付 & F & & $\mathscr{\infty}$ & & 守 \\
\hline 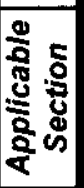 & 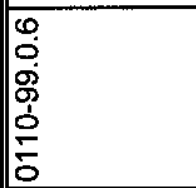 & 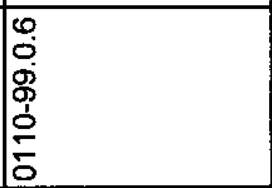 & 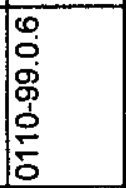 & 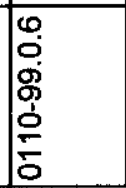 & $\begin{array}{l}0 \\
8 \\
8 \\
0 \\
0\end{array}$ & 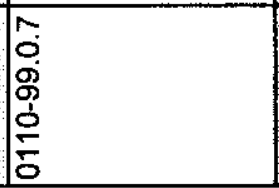 & g. & $\frac{\text { }}{0}$ \\
\hline
\end{tabular}




\begin{tabular}{|c|c|c|c|c|c|c|c|c|c|}
\hline 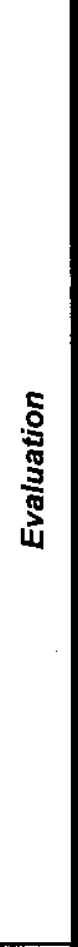 & 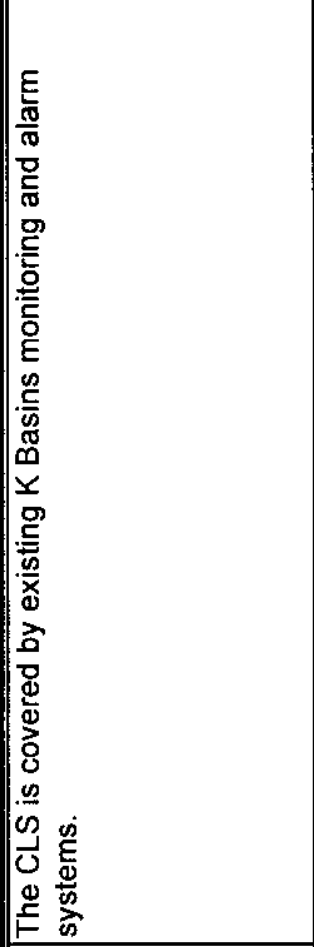 & 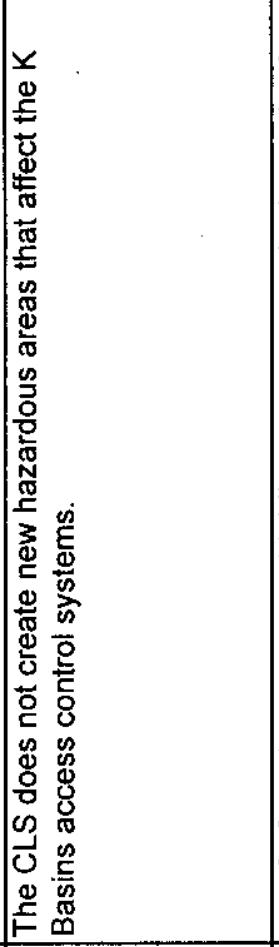 & 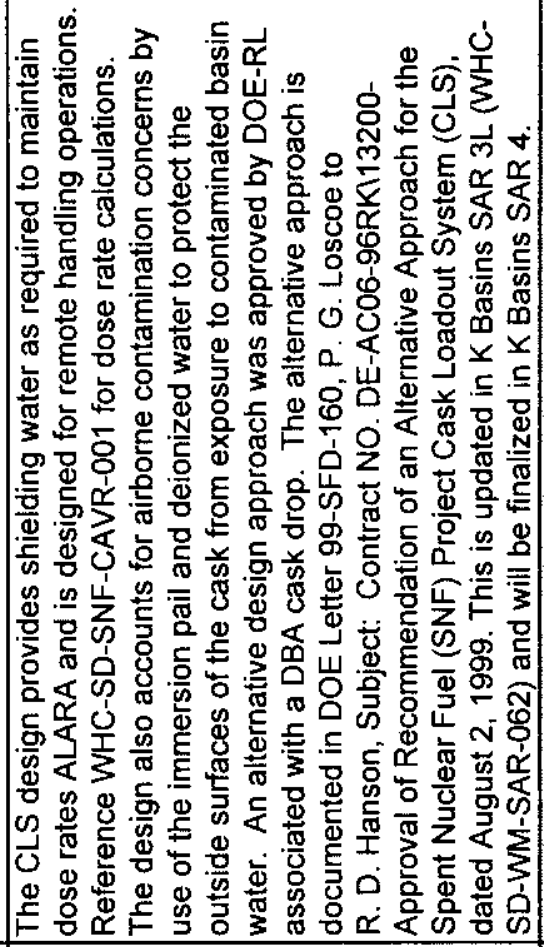 & & & 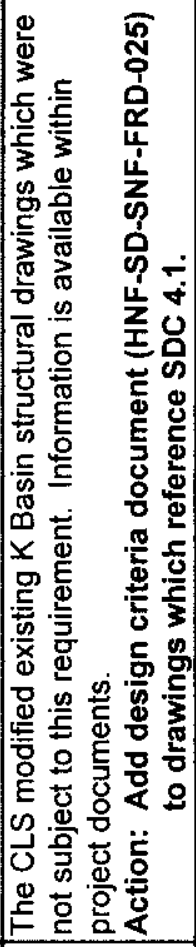 & 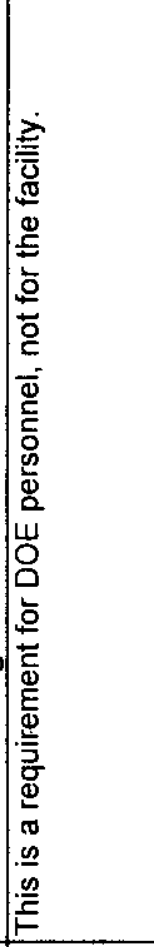 & & \\
\hline 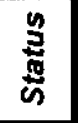 & 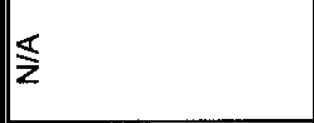 & 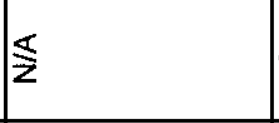 & 0 & & & $\begin{array}{l}z \\
\text { za } \\
0\end{array}$ & $\leqslant$ & & \\
\hline 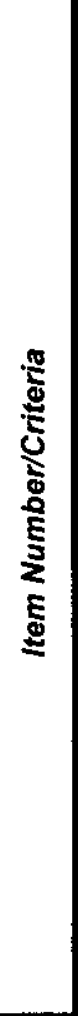 & 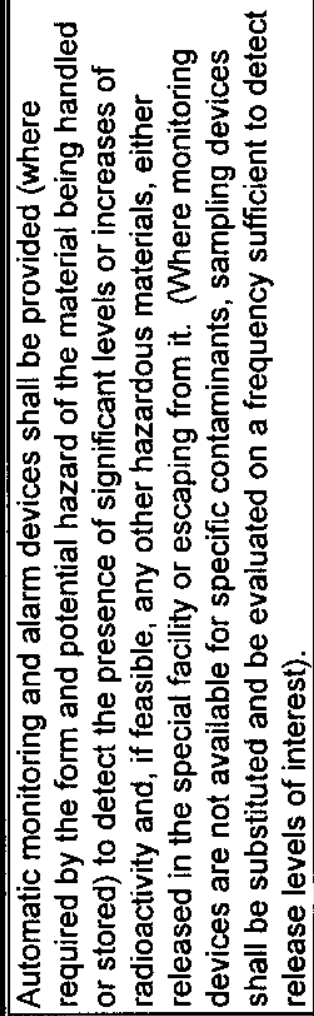 & 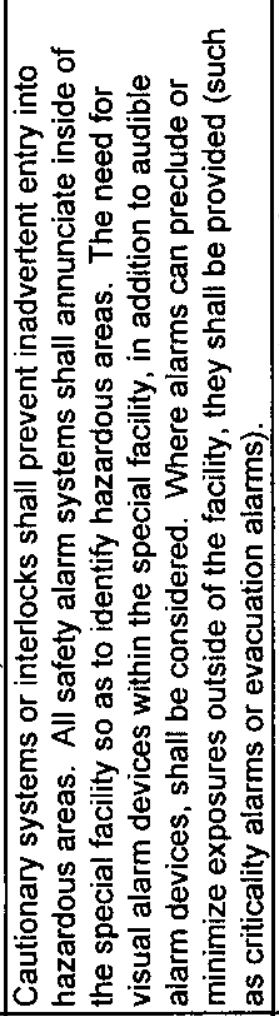 & 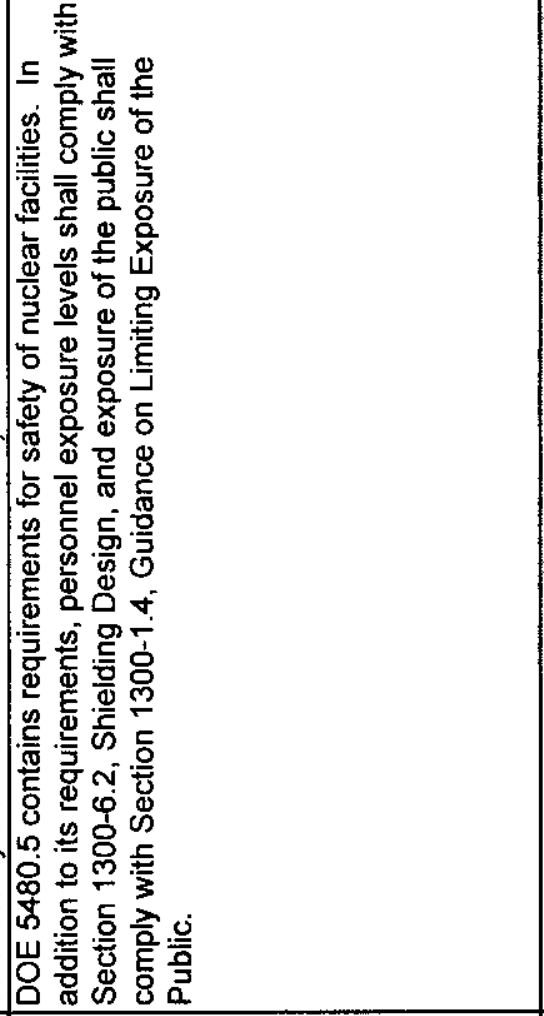 & 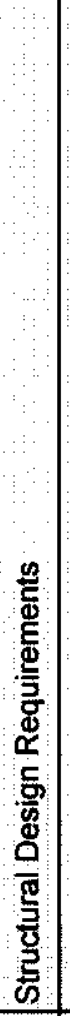 & $\begin{array}{l} \\
\\
\\
\\
5 \\
\frac{\pi}{9} \\
\frac{5}{5}\end{array}$ & 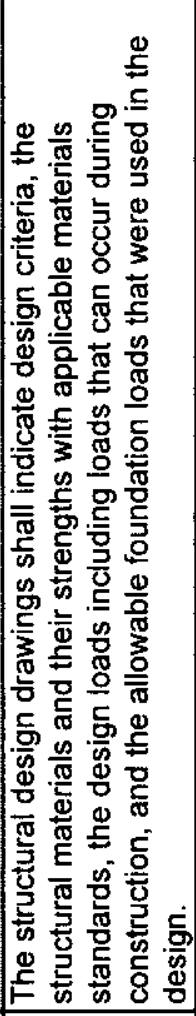 & 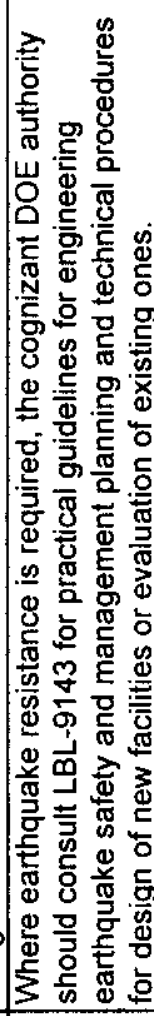 & 8 & 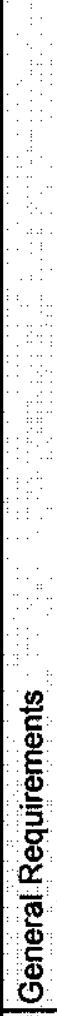 \\
\hline ¿ & 80 & in & กิ & & & గ్ర & 苗 & & \\
\hline 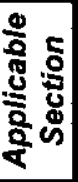 & $\begin{array}{l}\infty \\
0 \\
0 \\
0 \\
0 \\
0 \\
0 \\
\\
0\end{array}$ & 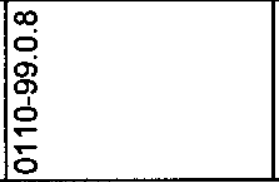 & 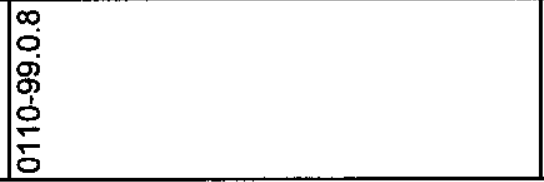 & 0 & 5 & $\frac{I}{5}$ & $\frac{I}{\check{E}}$ & $\frac{7}{6}$ & Еे \\
\hline
\end{tabular}


SNF-4775, Rev. 2

Project A.5/A.6

APPENDIX A

\begin{tabular}{|c|c|c|c|c|c|c|c|c|c|c|c|c|c|c|c|c|c|}
\hline 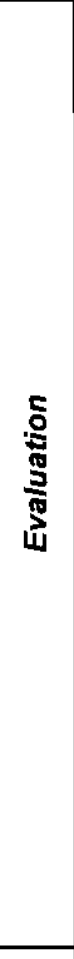 & 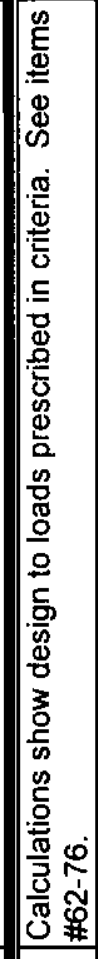 & & & 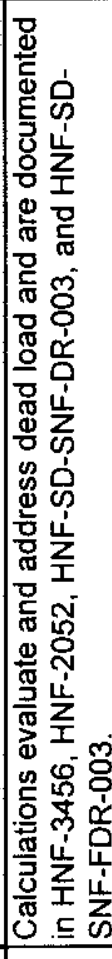 & 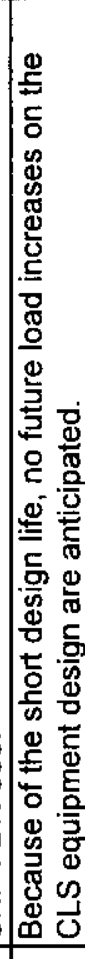 & & 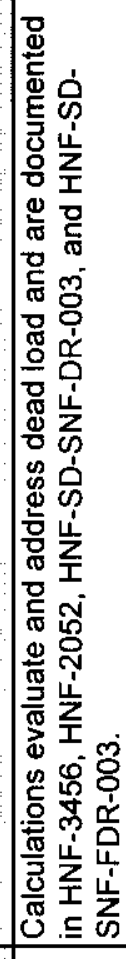 & & & 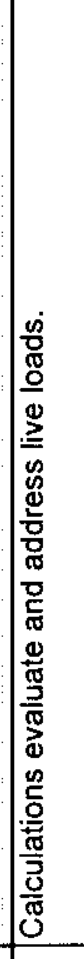 & 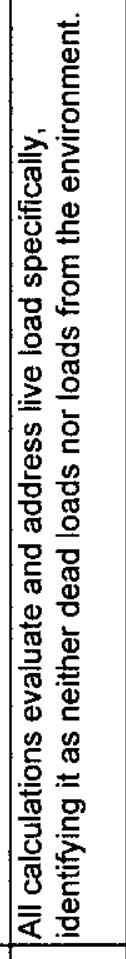 & & 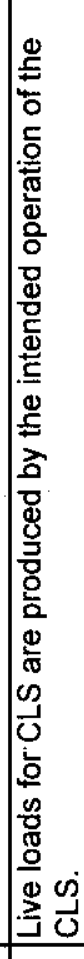 & 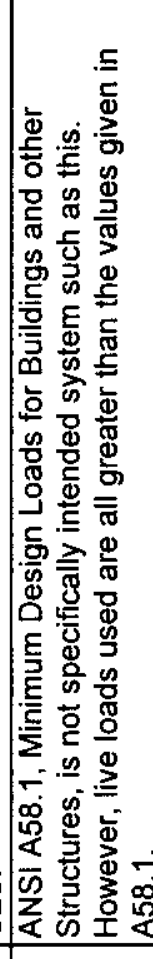 & 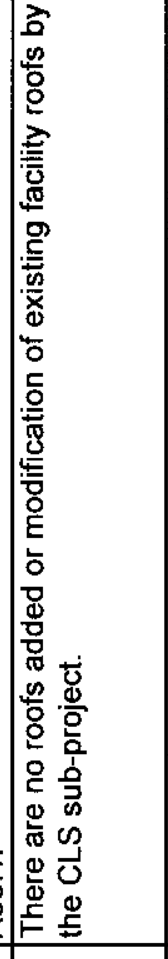 & 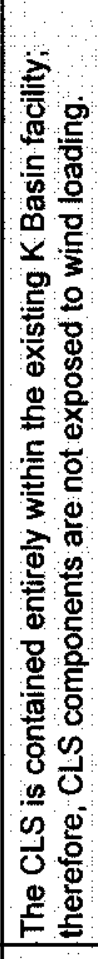 & 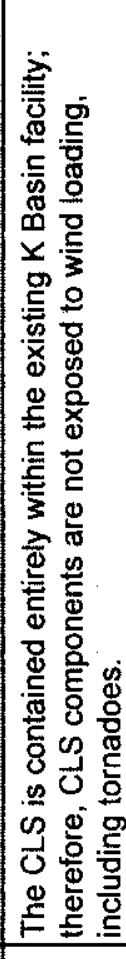 \\
\hline $\begin{array}{l}\text { 胥 } \\
\text { 品 }\end{array}$ & 0 & & & 0 & $\frac{\$}{\mathbf{z}}$ & & 0 & & & 0 & 0 & & 0 & $\stackrel{\$}{\mathbf{z}}$ & $\frac{\$}{\mathbf{z}}$ & $\frac{s}{z}$ & 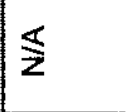 \\
\hline 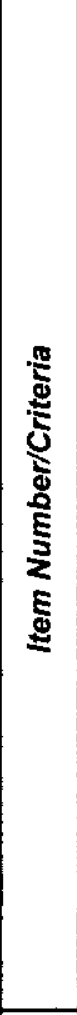 & 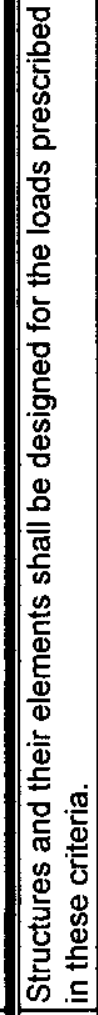 & $\begin{array}{l}0 \\
8 \\
3 \\
8 \\
8 \\
0\end{array}$ & $\begin{array}{l}\bar{\Xi} \\
\widetilde{0} \\
\end{array}$ & 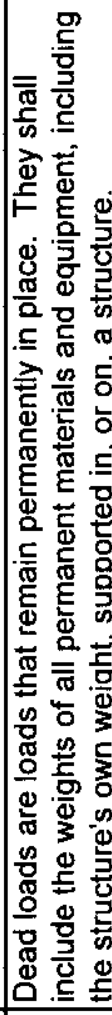 & 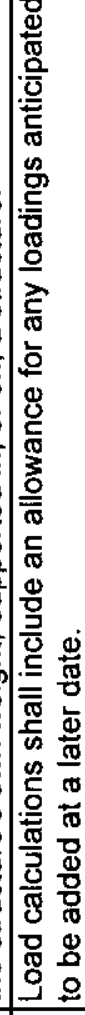 & 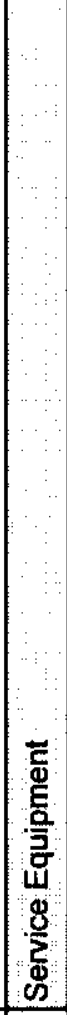 & 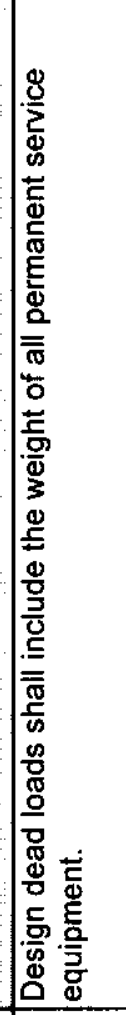 & $\begin{array}{l}0 \\
8 \\
0 \\
3 \\
0 \\
0\end{array}$ & $\frac{\pi}{8}$ & 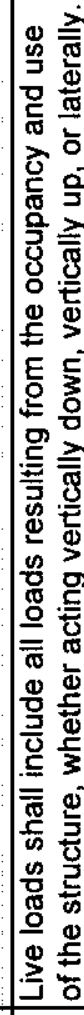 & 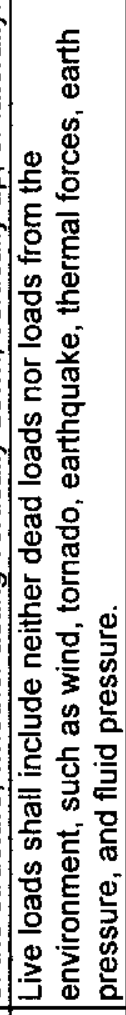 & 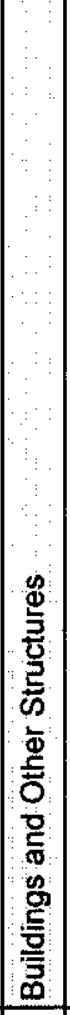 & 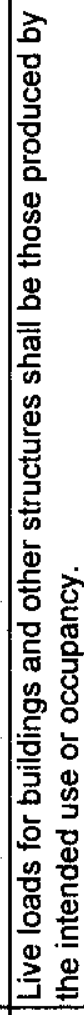 & 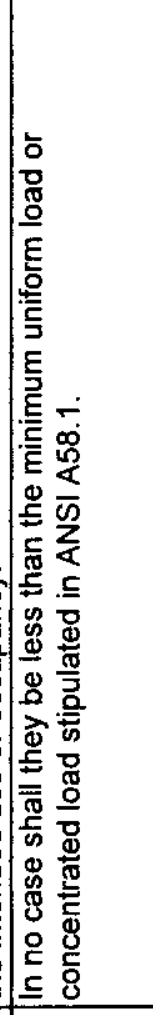 & 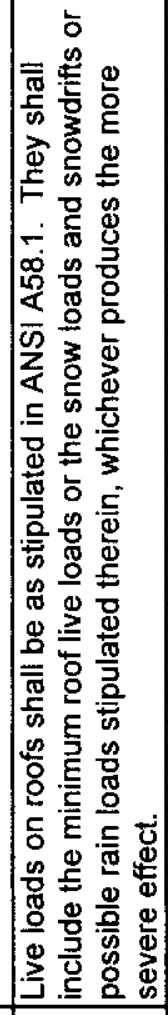 & $\begin{array}{l}0 \\
0 \\
0 \\
\\
\end{array}$ & \begin{tabular}{|l}
0 \\
0 \\
0 \\
0 \\
0 \\
0 \\
0 \\
0 \\
0 \\
0 \\
0 \\
0 \\
0
\end{tabular} \\
\hline$\dot{2}$ & 造 & & & 10 & $\sqrt{n}$ & & 曲 & & & ) & 80 & & $\overline{0}$ & ชิ & 8 & 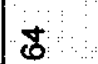 & 18 \\
\hline 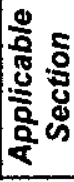 & 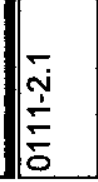 & $\frac{N}{2}$ & & $\underset{\substack{N\\
}}{\stackrel{N}{\sigma}}$ & 岕 & ल & $\underset{\substack{\tilde{N}\\
}}{\frac{N}{\sigma}}$ & $\frac{\pi}{2}$ & $\frac{\tilde{m}}{\tilde{N}}$ & 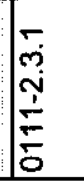 & $\underset{\substack{\tilde{N} \\
\stackrel{\sim}{\sigma}}}{\frac{\sigma}{0}}$ & {$\left[\begin{array}{c}N \\
n \\
N \\
\\
\\
0\end{array}\right.$} & 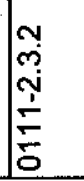 & 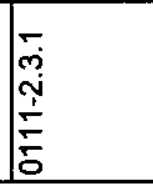 & 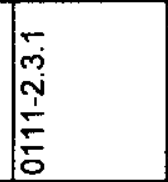 & $\frac{i}{\frac{1}{5}}$ & 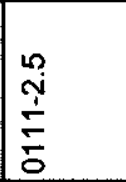 \\
\hline
\end{tabular}

Page A-9 
SNF-4775, Rev. 2

Project A.5/A.6

APPENDIX A

\begin{tabular}{|c|c|c|c|c|c|c|c|c|c|c|c|c|}
\hline | & 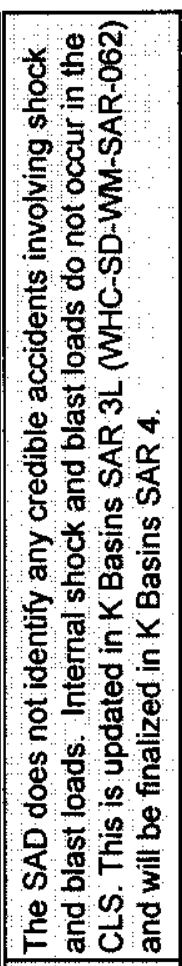 & & & 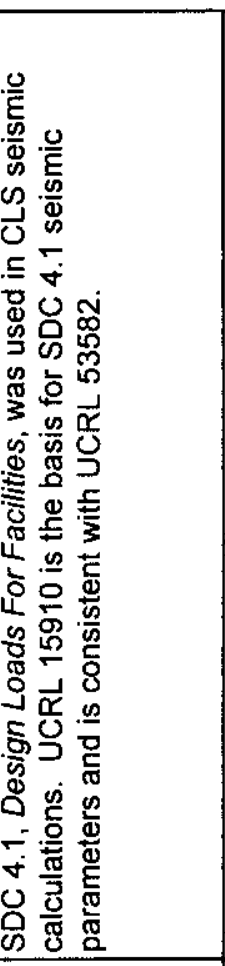 & 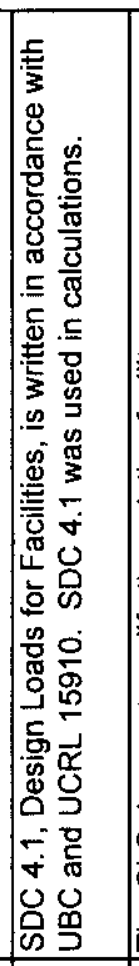 & 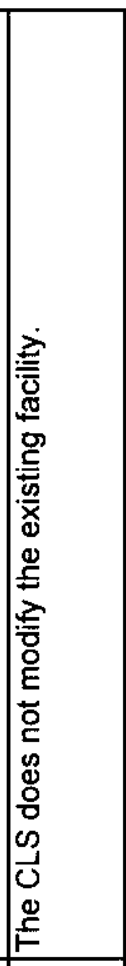 & 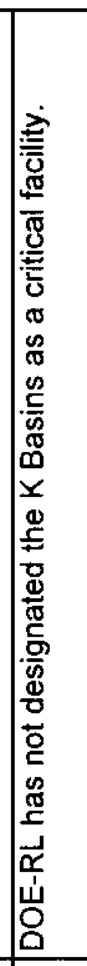 & 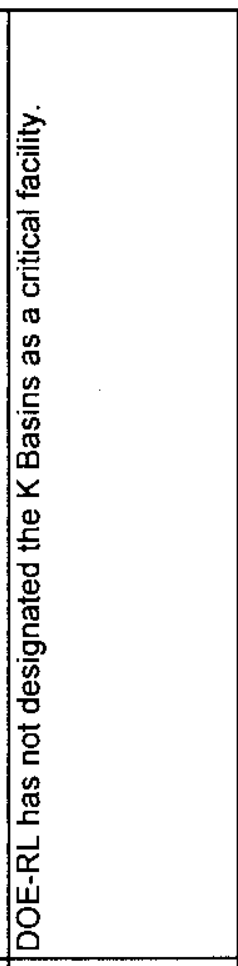 & 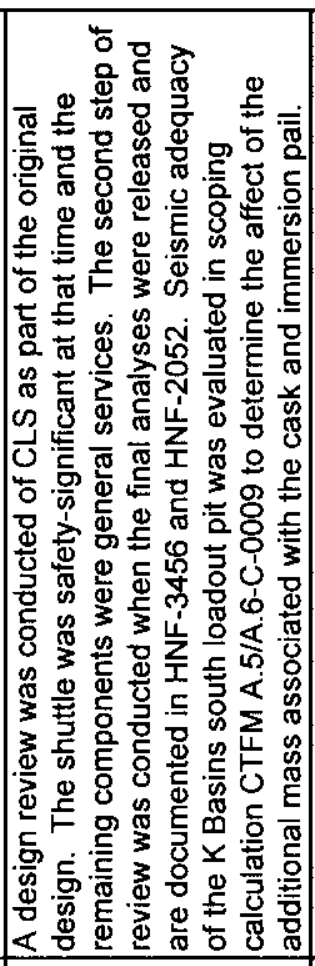 & & 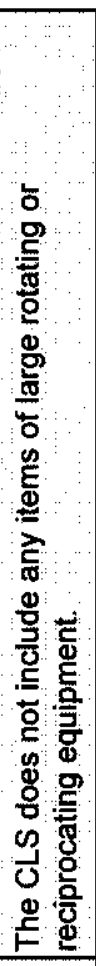 & 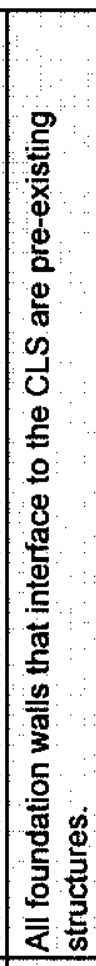 \\
\hline 胥 & $\frac{\varsigma}{z}$ & & & 0 & 0 & $\S$ & $\mid \frac{\widehat{\Sigma}}{\mathbf{z}}$ & $\underline{\mathbf{z}}$ & 0 & & $\frac{\varsigma}{\mathbf{z}}$ & 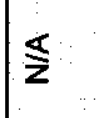 \\
\hline 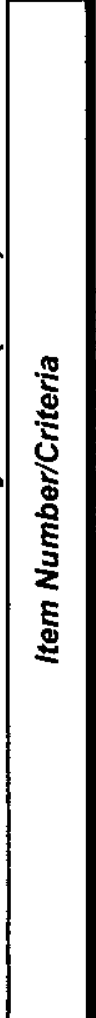 & 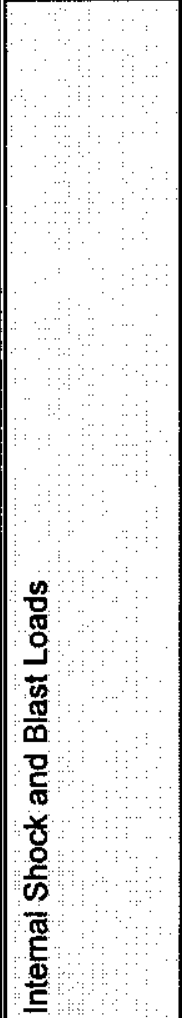 & 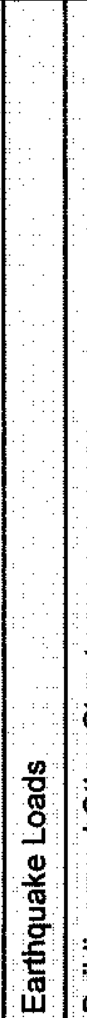 & 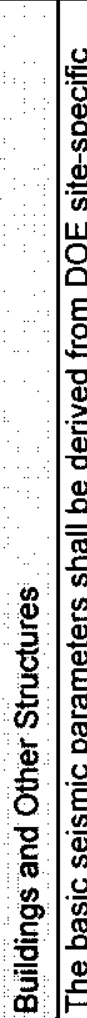 & 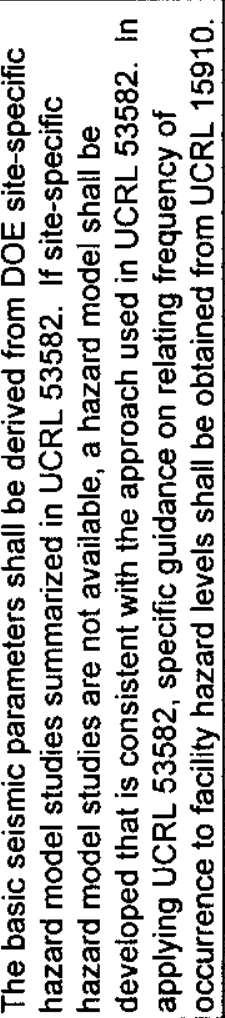 & 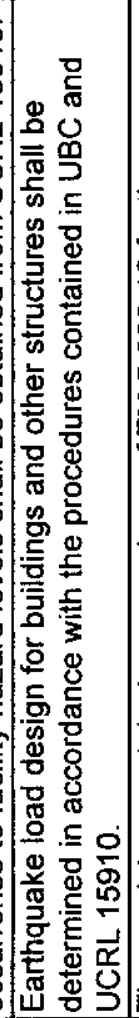 & 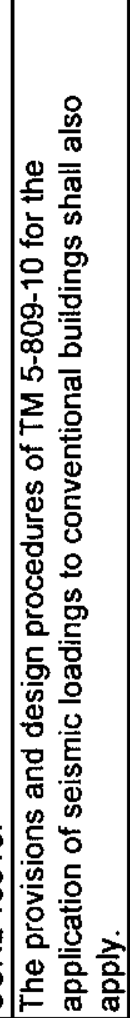 & 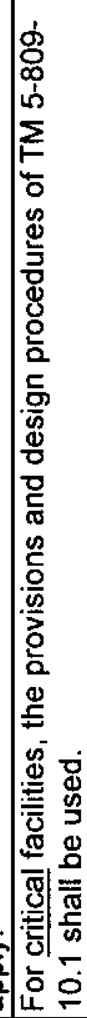 & 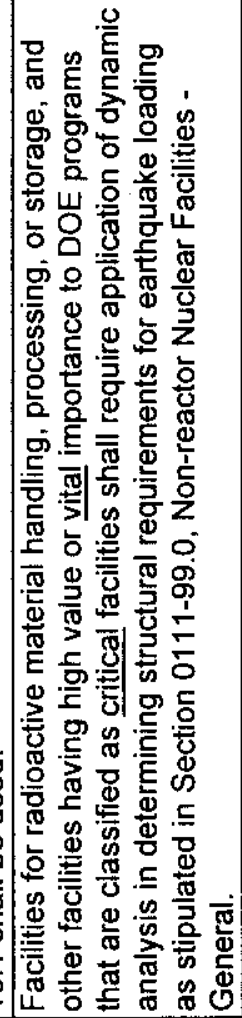 & 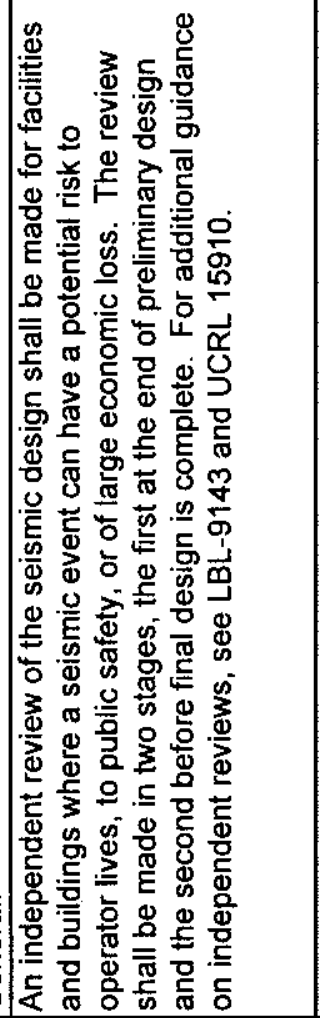 & 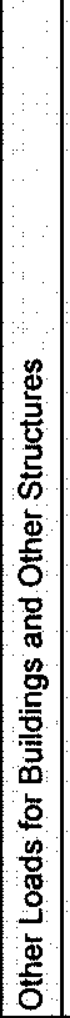 & $\begin{array}{l}\frac{0}{5} \\
\frac{5}{0} \\
0 \\
\frac{0}{5} \\
\frac{0}{5} \\
\frac{5}{5}\end{array}$ & 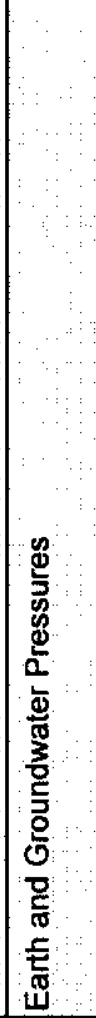 \\
\hline i & 8 & & & $\widehat{\epsilon}$ & 10 & 8 & i & $\pi$ & 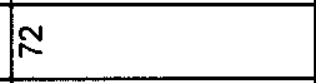 & & s & 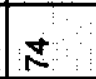 \\
\hline 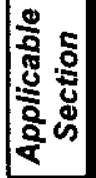 & $\stackrel{\leftrightarrow}{\stackrel{\circ}{ }}$ & $\frac{\hat{N}}{\bar{\delta}}$ & $\frac{\pi}{\sigma}=$ & & 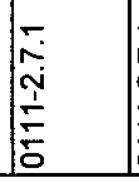 & 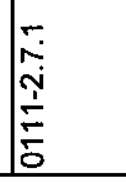 & 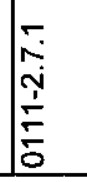 & 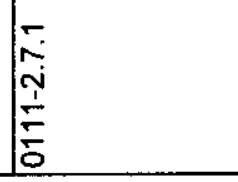 & 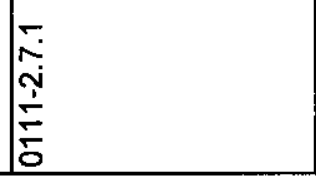 & $\begin{array}{c}\infty \\
\stackrel{2}{2} \\
\stackrel{5}{b}\end{array}$ & $\frac{\infty}{2}$ & 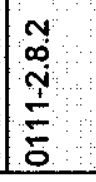 \\
\hline
\end{tabular}


SNF-4775, Rev. 2

Project A.5/A.6

APPENDIX A

\begin{tabular}{|c|c|c|c|c|c|c|c|c|c|c|c|}
\hline 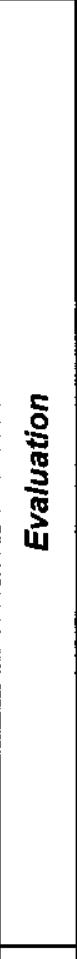 & 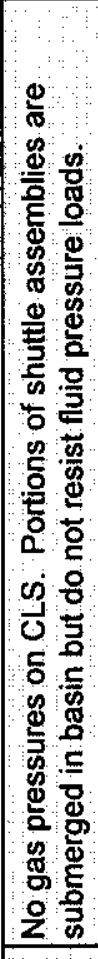 & 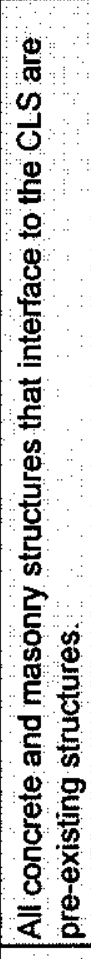 & & 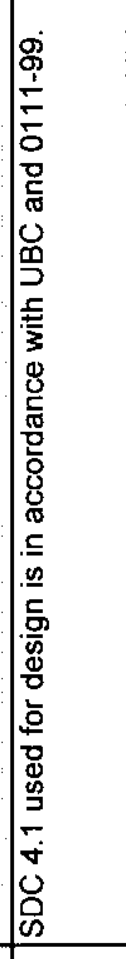 & 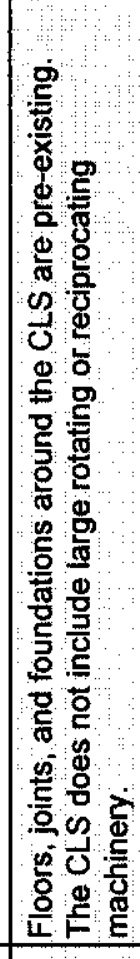 & & & & & 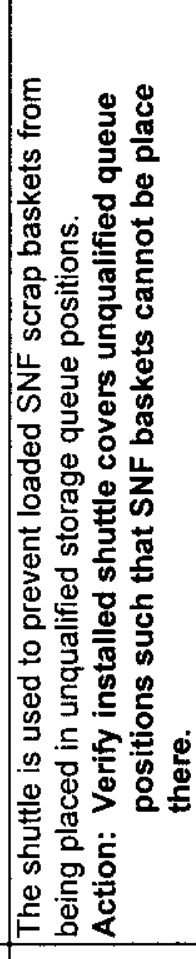 & 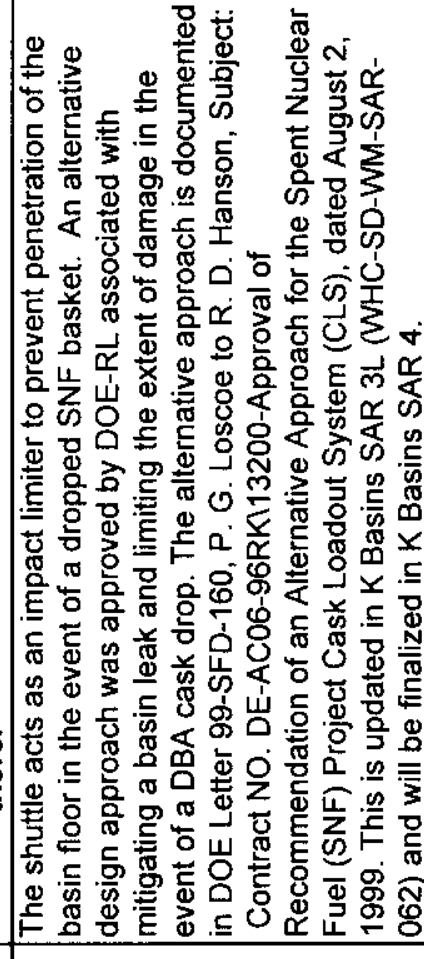 \\
\hline 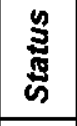 & $\underset{z}{\mathbf{z}}$ & $\frac{\widehat{z}}{\mathbf{z}}$ & & 0 & $\underset{z}{\mathbf{z}}$ & & & & & zu & 0 \\
\hline 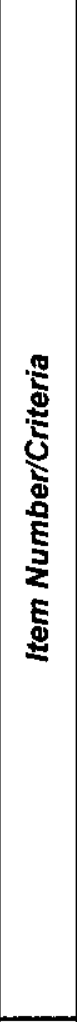 & 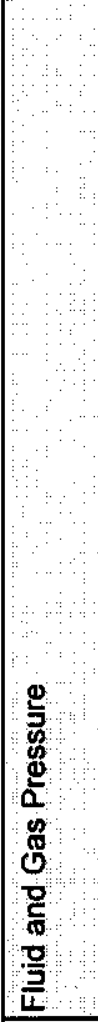 & $\begin{array}{l}0 \\
8 \\
0 \\
0 \\
0 \\
0 \\
\frac{0}{5} \\
0 \\
0 \\
0 \\
0 \\
0\end{array}$ & 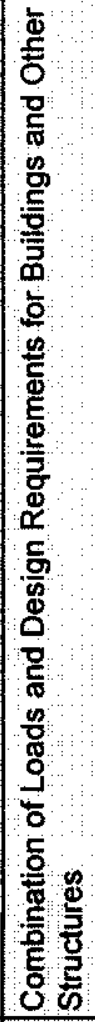 & 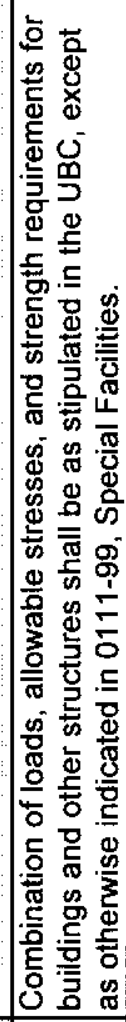 & 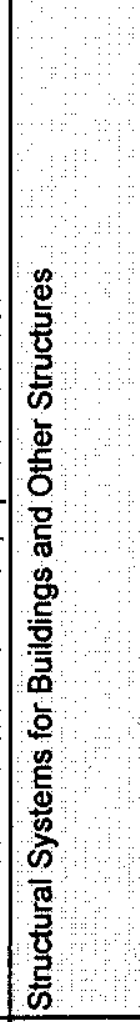 & 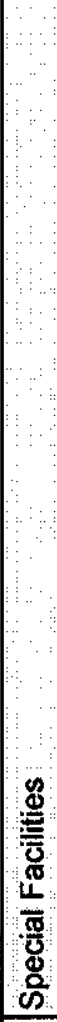 & 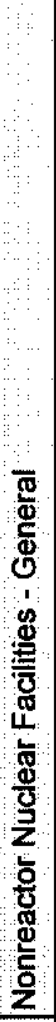 & 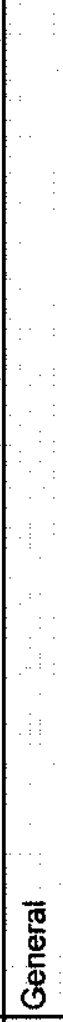 & 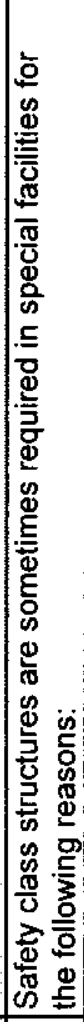 & 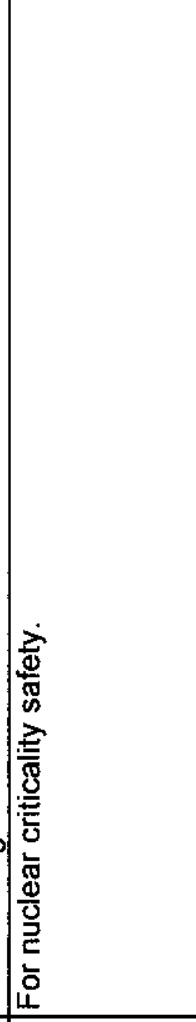 & 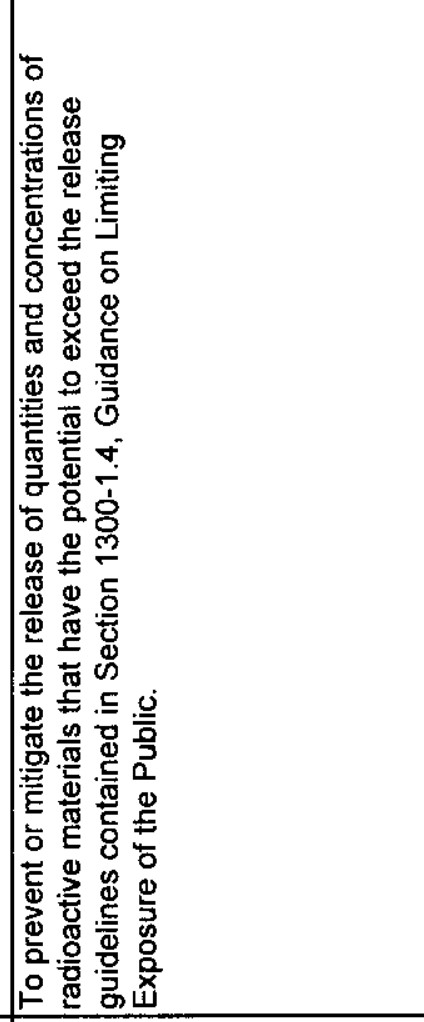 \\
\hline$i^{2}$ & $\stackrel{2}{2}$ & 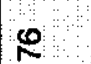 & & $\mathbb{N}$ & $\propto$ & & & & $R$ & ळం & $\bar{\infty}_{\infty}$ \\
\hline 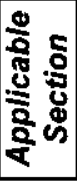 & 足 & {$\left[\begin{array}{l}\infty \\
\tilde{\delta}\end{array}\right.$} & $\frac{0}{\tilde{2}}$ & 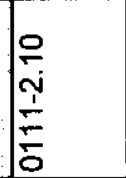 & $\frac{2}{5}$ & $\frac{1}{6}$ & $\stackrel{8}{\circ}$ & $\begin{array}{l}\overline{0} \\
\overline{8} \\
\overline{8} \\
\overline{1}\end{array}$ & 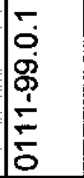 & 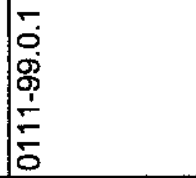 & 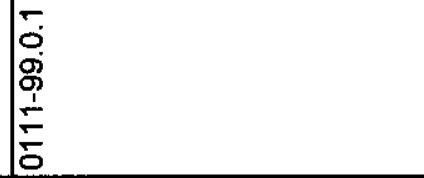 \\
\hline
\end{tabular}


SNF-4775, Rev. 2

Project A.5/A.6

APPENDIX A

\begin{tabular}{|c|c|c|c|c|c|c|c|c|c|c|c|}
\hline 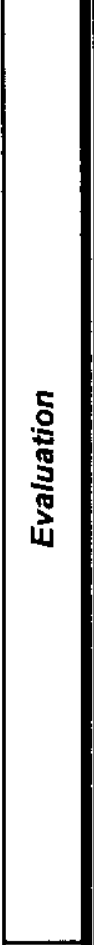 & 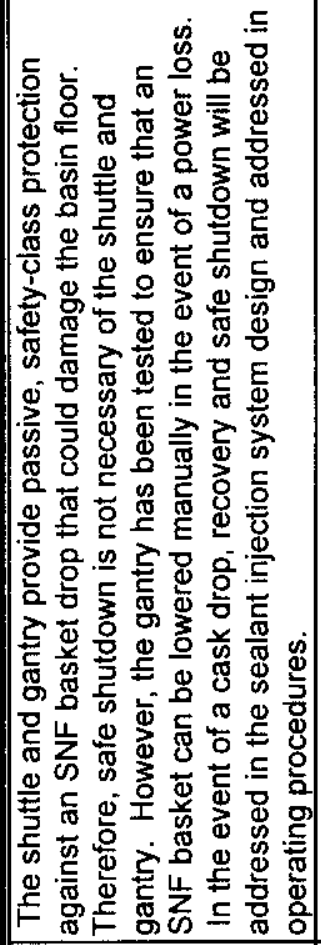 & 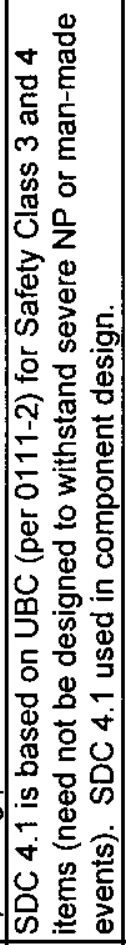 & 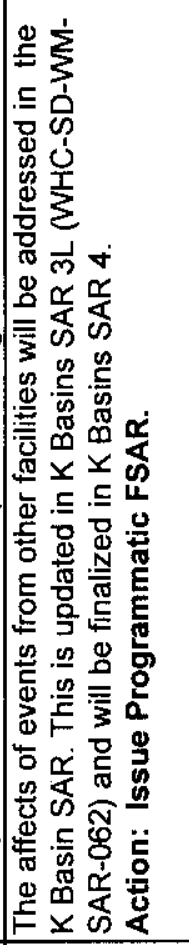 & 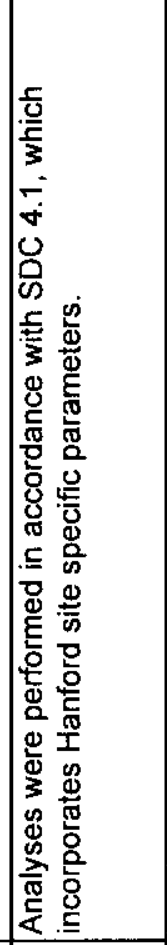 & 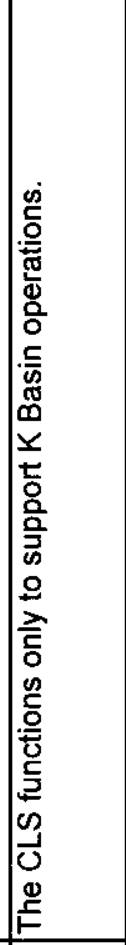 & 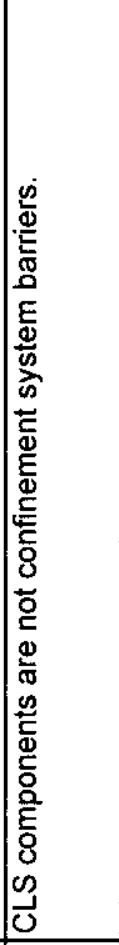 & 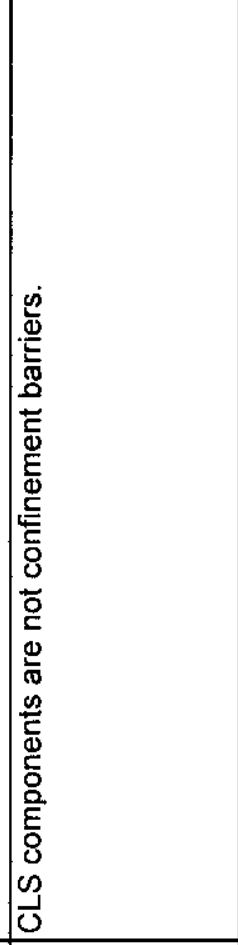 & 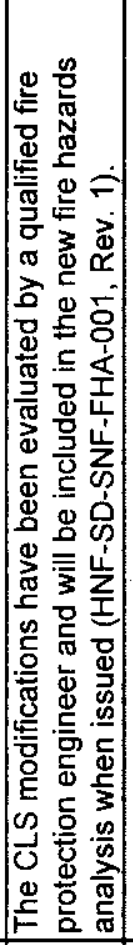 & 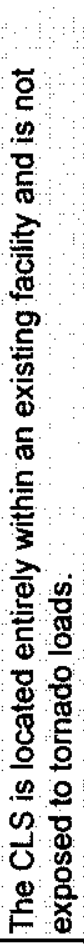 & 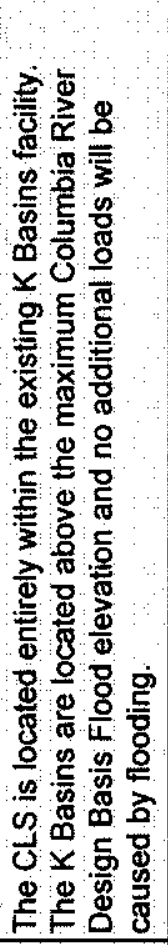 & \\
\hline 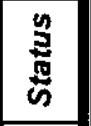 & 0 & 0 & 岕 & 0 & $\frac{1}{z}$ & $\$$ & $\frac{\aleph}{z}$ & 0 & $\frac{\mathbb{Z}}{\mathbf{z}}$ & 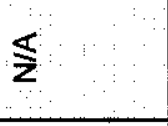 & \\
\hline 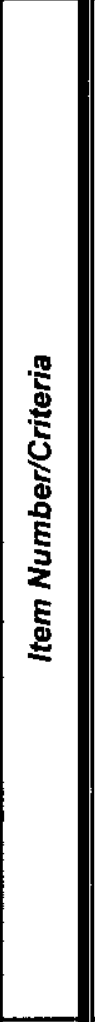 & 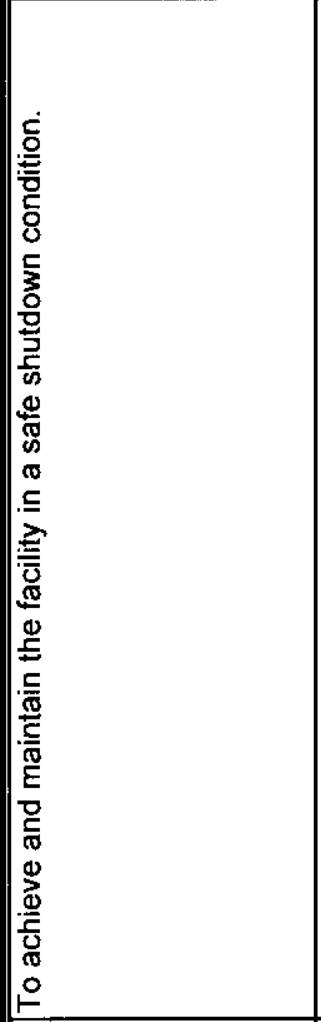 & 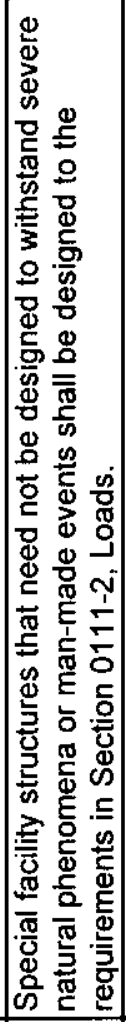 & 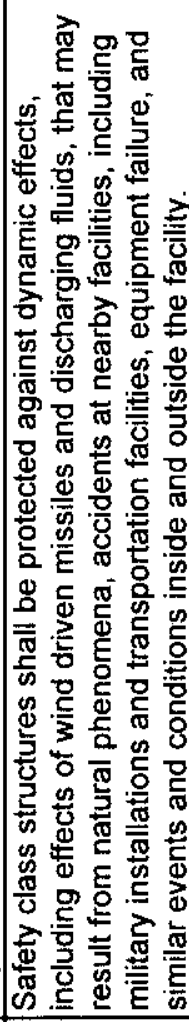 & 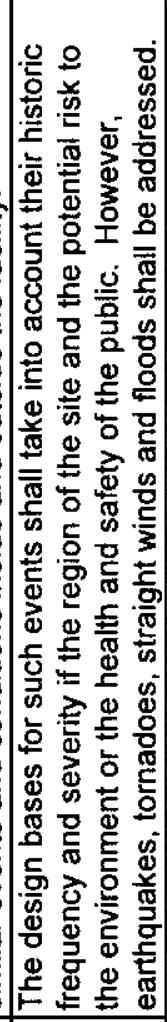 & 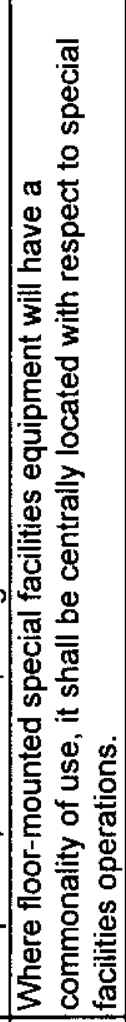 & 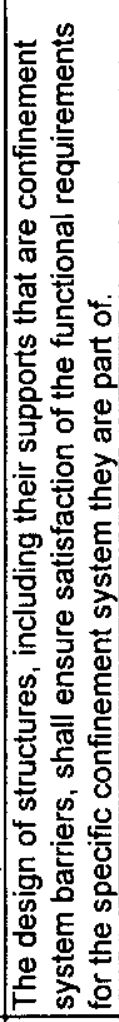 & 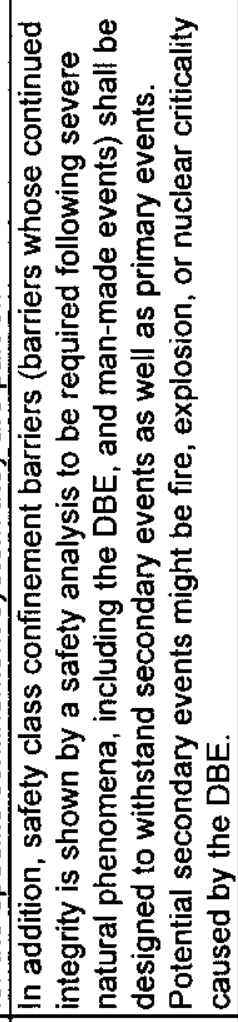 & 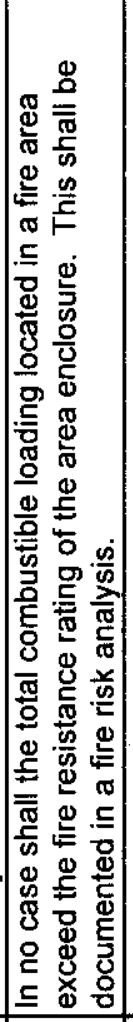 & 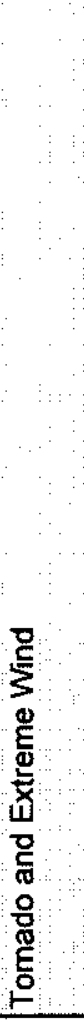 & $\frac{\infty}{8}$ & 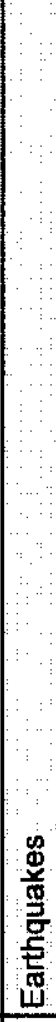 \\
\hline z & $\infty$ & $\infty$ & 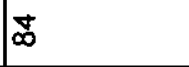 & 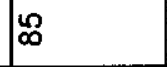 & 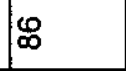 & $1 \infty$ & $\infty$ & $\infty$ & \& & $\delta$ & \\
\hline 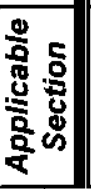 & 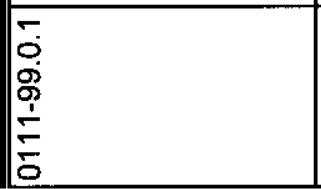 & 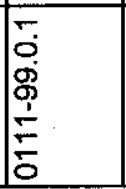 & 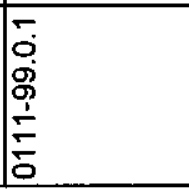 & 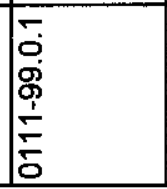 & 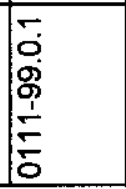 & 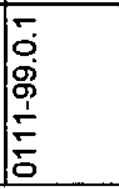 & 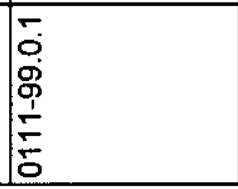 & 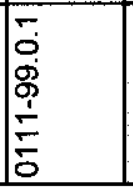 & $\begin{array}{l} \\
0 \\
8 \\
\\
\\
\\
\end{array}$ & ह & $\begin{array}{l}\overline{1} \\
0 \\
0 \\
1 \\
\\
\end{array}$ \\
\hline
\end{tabular}


SNF-4775, Rev. 2

Project A.5/A.6

APPENDIX A

\begin{tabular}{|c|c|c|c|c|c|c|c|c|c|c|c|c|c|c|c|}
\hline 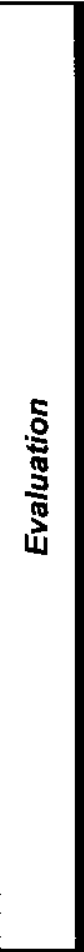 & & 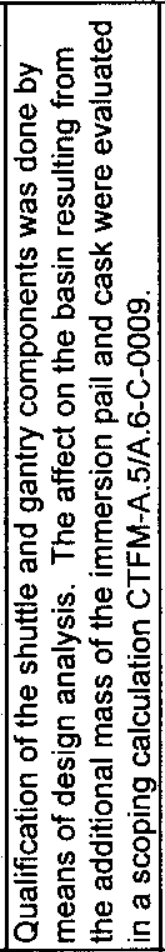 & 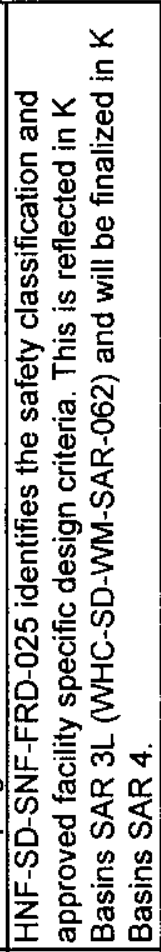 & 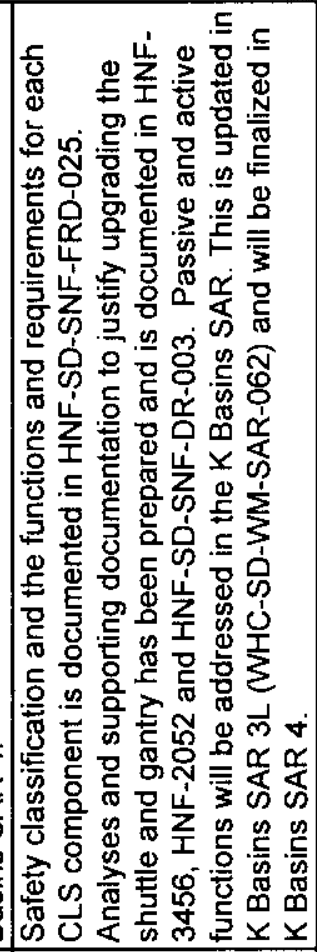 & 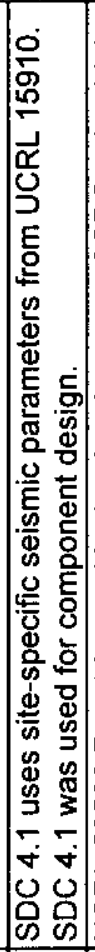 & 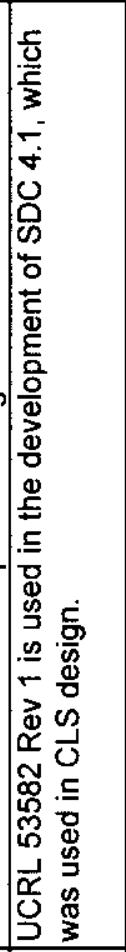 & 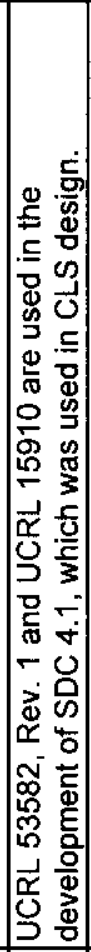 & 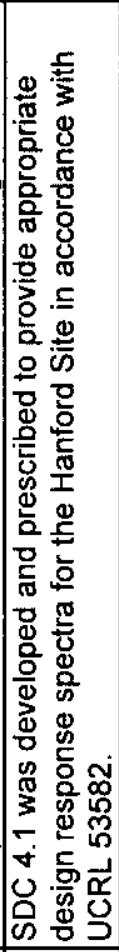 & 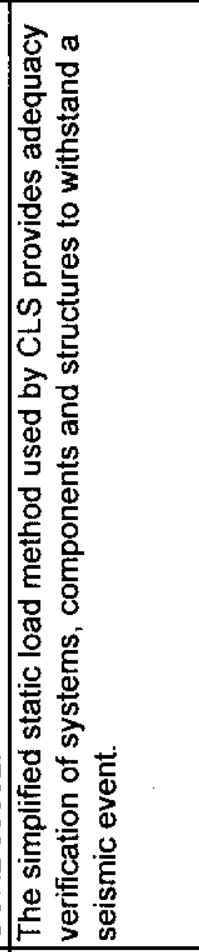 & 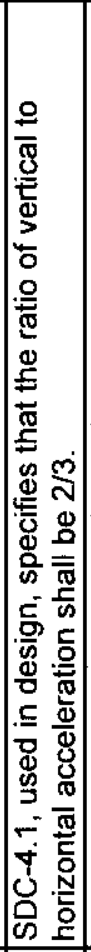 & 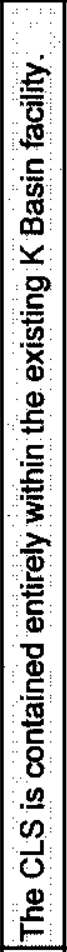 & 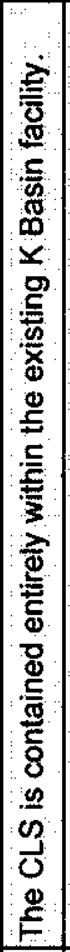 & 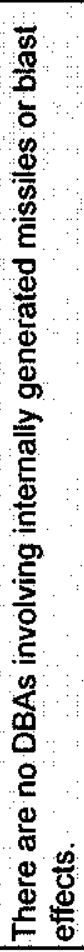 & & 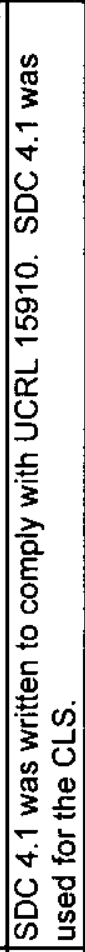 \\
\hline 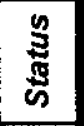 & & $\$$ & 0 & 0 & 0 & 0 & 0 & 0 & 0 & 0 & $\stackrel{\mathbf{z}}{\mathbf{z}}$ & $\$$ & $\$$ & & 0 \\
\hline 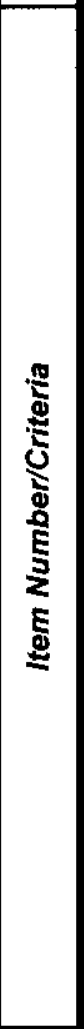 & 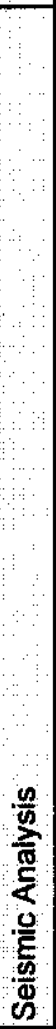 & 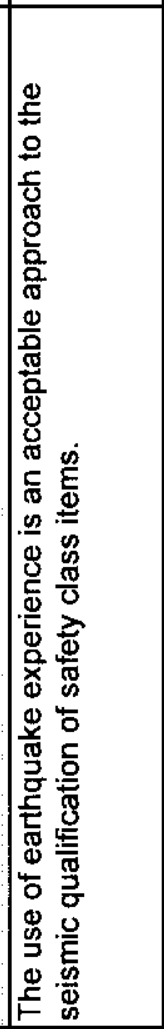 & 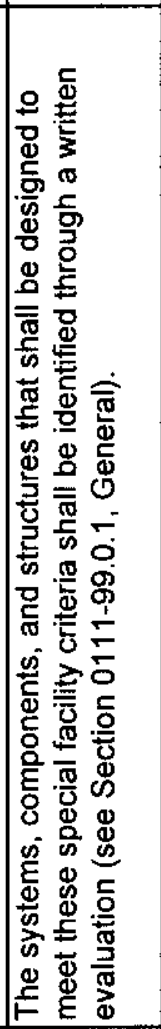 & 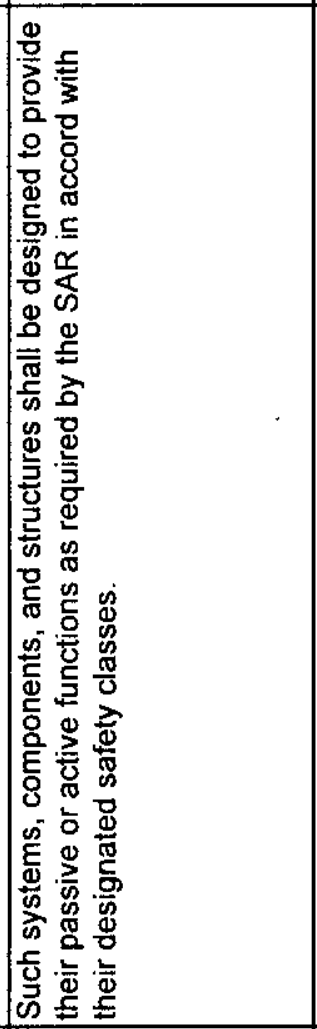 & 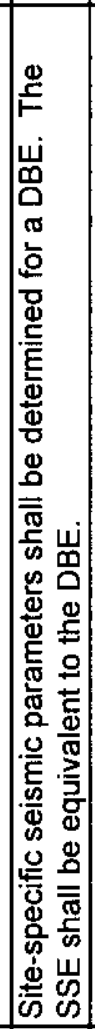 & 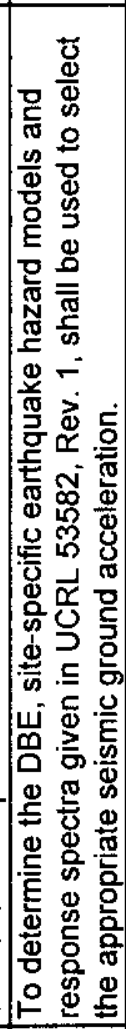 & 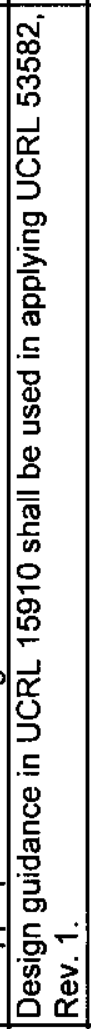 & 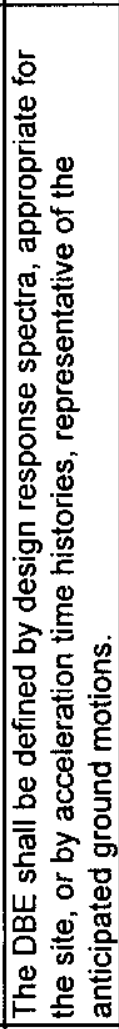 & 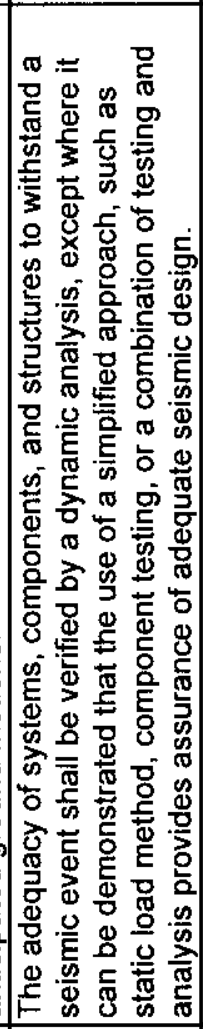 & 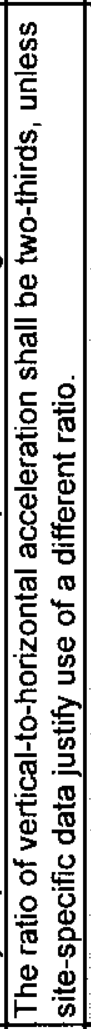 & $\frac{8}{8}$ & 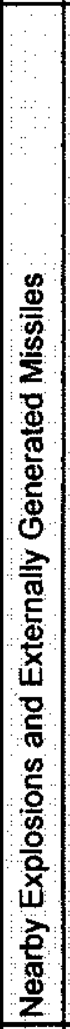 & 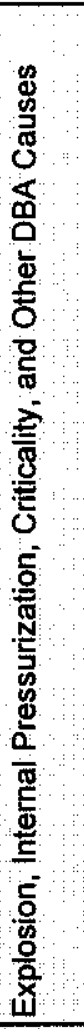 & 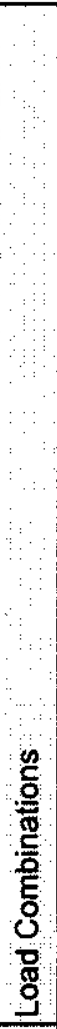 & 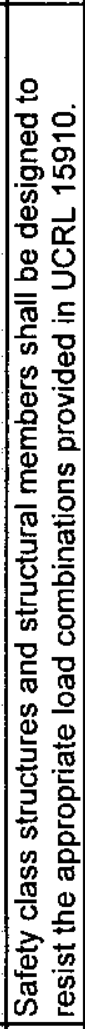 \\
\hline$\sum^{\circ}$ & & శ్ & హె & ঊ & \% & $\mathscr{S}$ & ô & 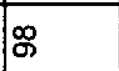 & |৪্ঞ & 8 & 으 & O & 8 & & 음 \\
\hline . & & & 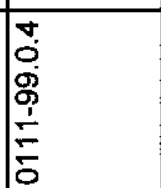 & 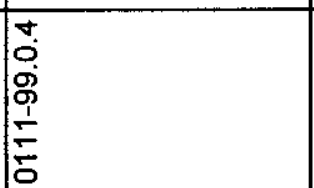 & & 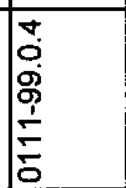 & 蒿 & O্் & |க் & \% & $\begin{array}{l}8 \\
\text { g } \\
\frac{3}{6}\end{array}$ & $\begin{array}{l}0 \\
0 \\
\\
\\
\end{array}$ & $\begin{array}{l}8 \\
\stackrel{8}{\circ} \\
\frac{8}{6}\end{array}$ & $\frac{0}{8}$ & 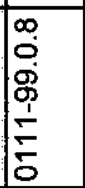 \\
\hline
\end{tabular}


SNF-4775, Rev. 2

Project A.5/A.6

APPENDIX A

\begin{tabular}{|c|c|c|c|c|c|c|c|c|c|c|c|c|c|c|c|c|c|}
\hline 宽 & 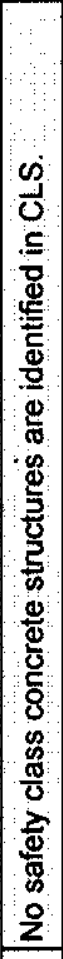 & & 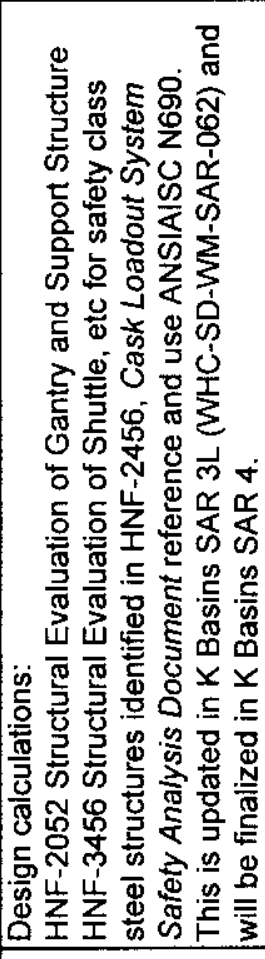 & & 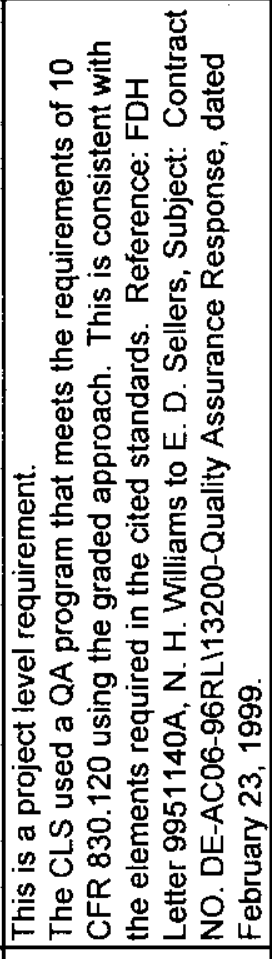 & 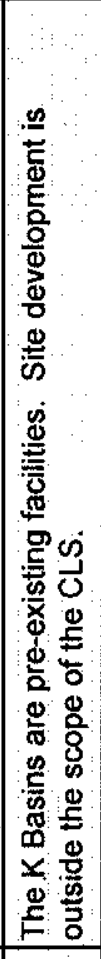 & 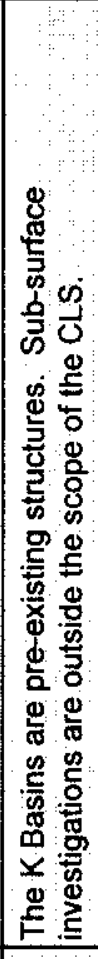 & 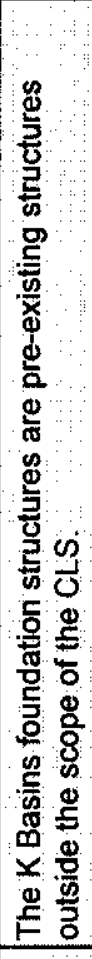 & 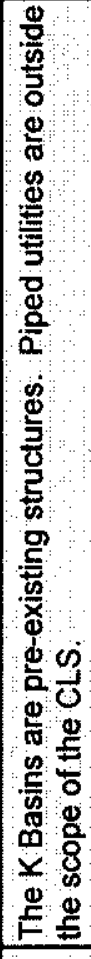 & & 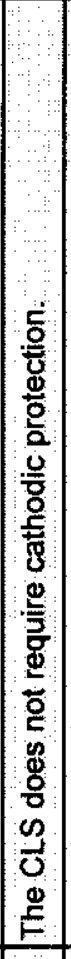 & 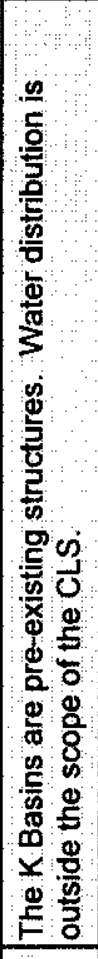 & 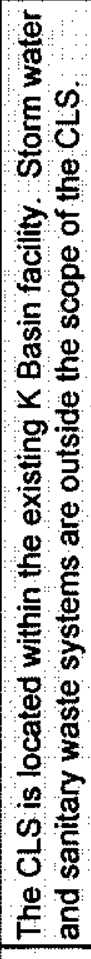 & 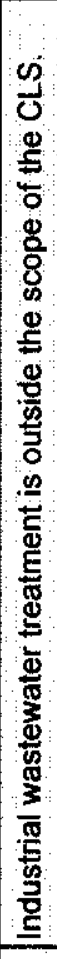 & 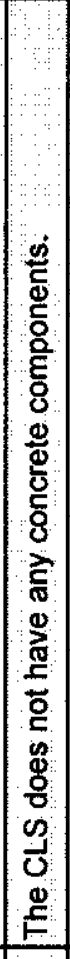 & 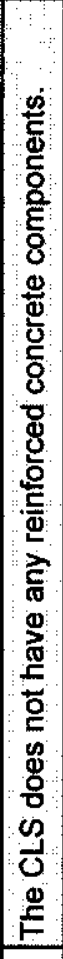 & 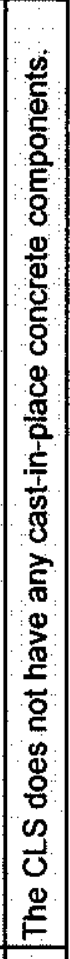 \\
\hline $\begin{array}{l}\text { 荧 } \\
\text { 心 } \\
\text { 心 }\end{array}$ & 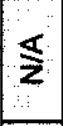 & & 0 & & $\S$ & $\underset{\mathbf{z}}{\mathbf{z}}$ & $\frac{\$}{z}$ & $\underset{z}{z}$ & 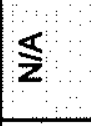 & & $\frac{5}{z}$ & $\widehat{\Sigma}$ & $\$$ & 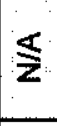 & $\$$ & 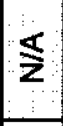 & $\$$ \\
\hline 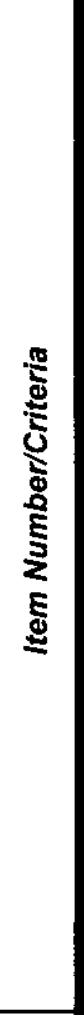 & 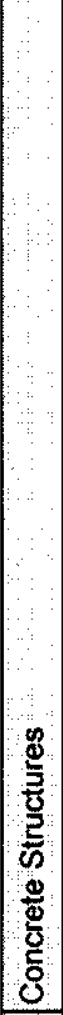 & 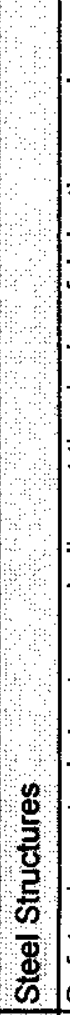 & 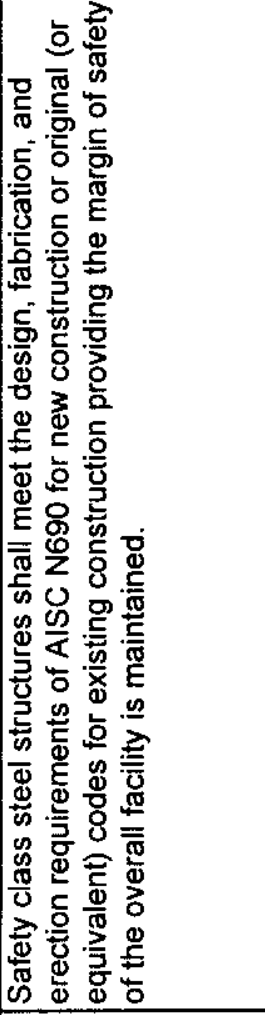 & 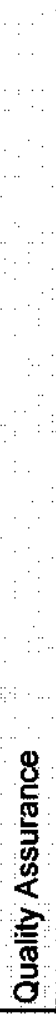 & 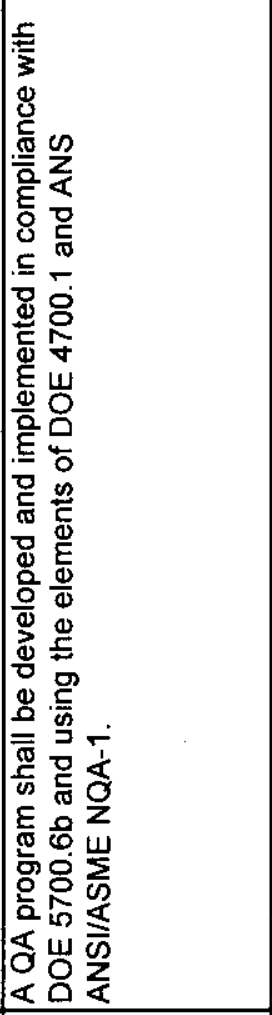 & 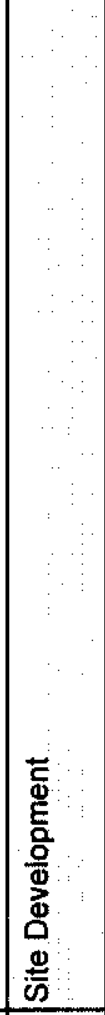 & 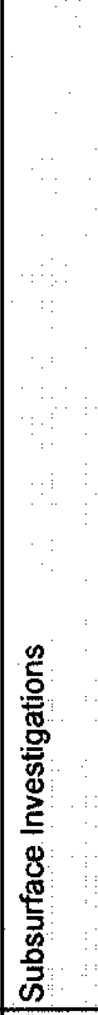 & 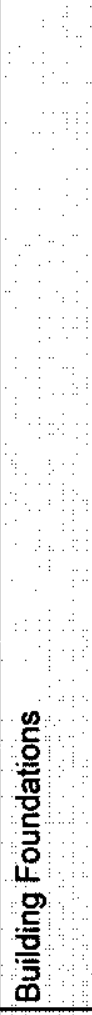 & 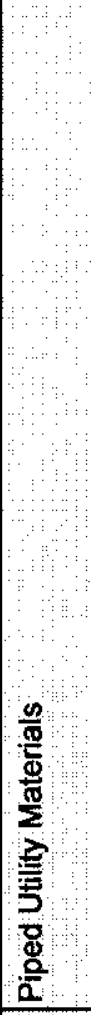 & $\begin{array}{c}\overline{0} \\
8 \\
0 \\
5 \\
0 \\
8 \\
\end{array}$ & 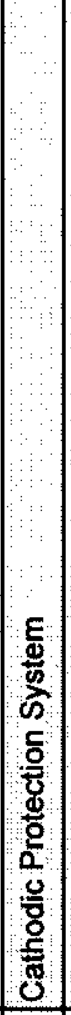 & 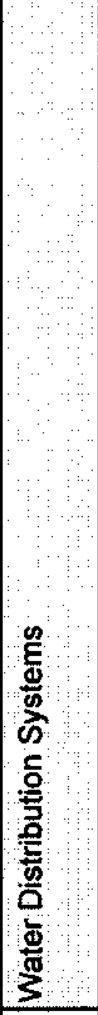 & 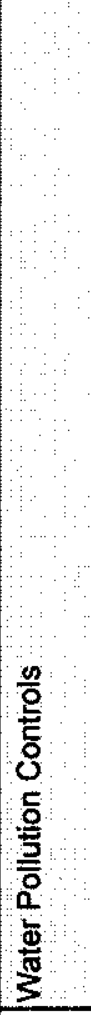 & 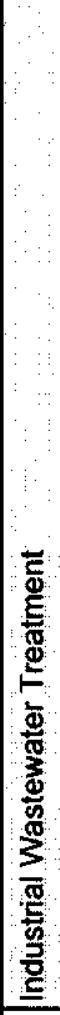 & 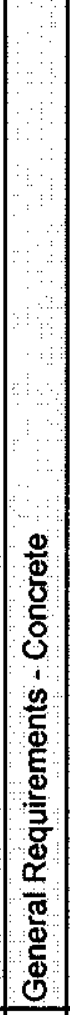 & 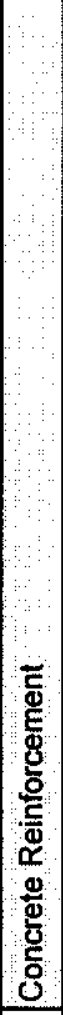 & 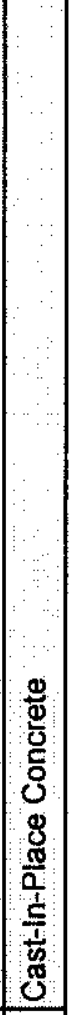 \\
\hline$\stackrel{0}{2}$ & 8 & & 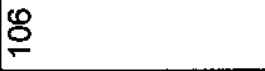 & & 응 & $\stackrel{8}{\circ}$ & 8 & 운 & $=$ & & $\stackrel{\sim}{*}$ & $\stackrel{m}{=}$ & \pm & 2 & $\stackrel{\circ}{=}$ & 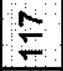 & $\stackrel{\infty}{=}$ \\
\hline 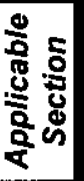 & क & $\begin{array}{c}\infty \\
8 \\
0 \\
0\end{array}$ & 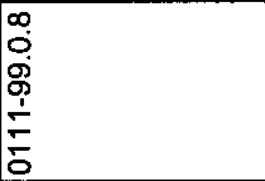 & 8 & 5 & ్్ㅇ & \&్ & ชิ & 8 & 8 & : & ช్ & 8 & 8 & 8 & 징 & ల్లి \\
\hline
\end{tabular}


SNF-4775, Rev. 2

Project A.5/A.6

APPENDIX A

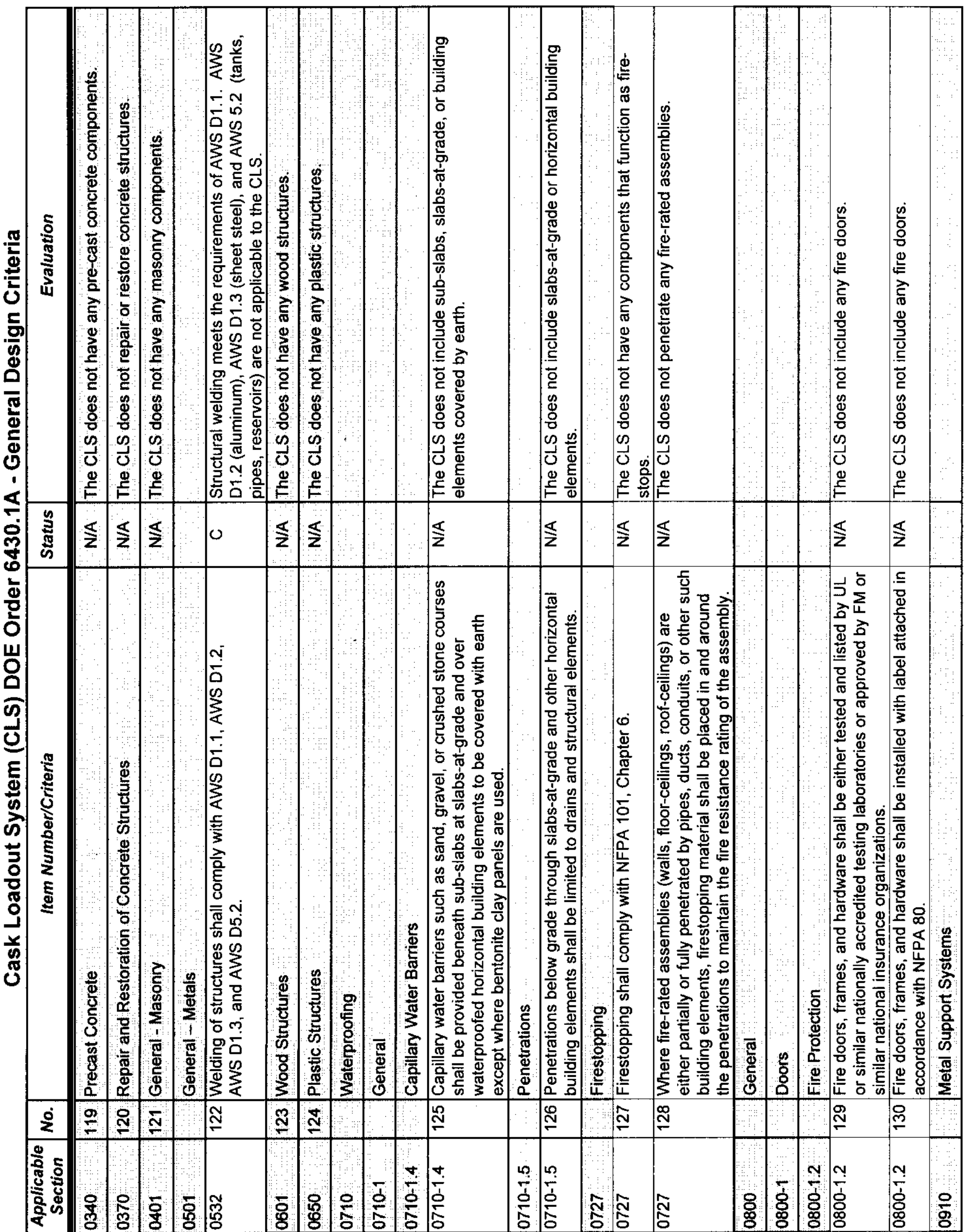


SNF-4775, Rev. 2

Project A.5/A.6

\section{APPENDIX A}

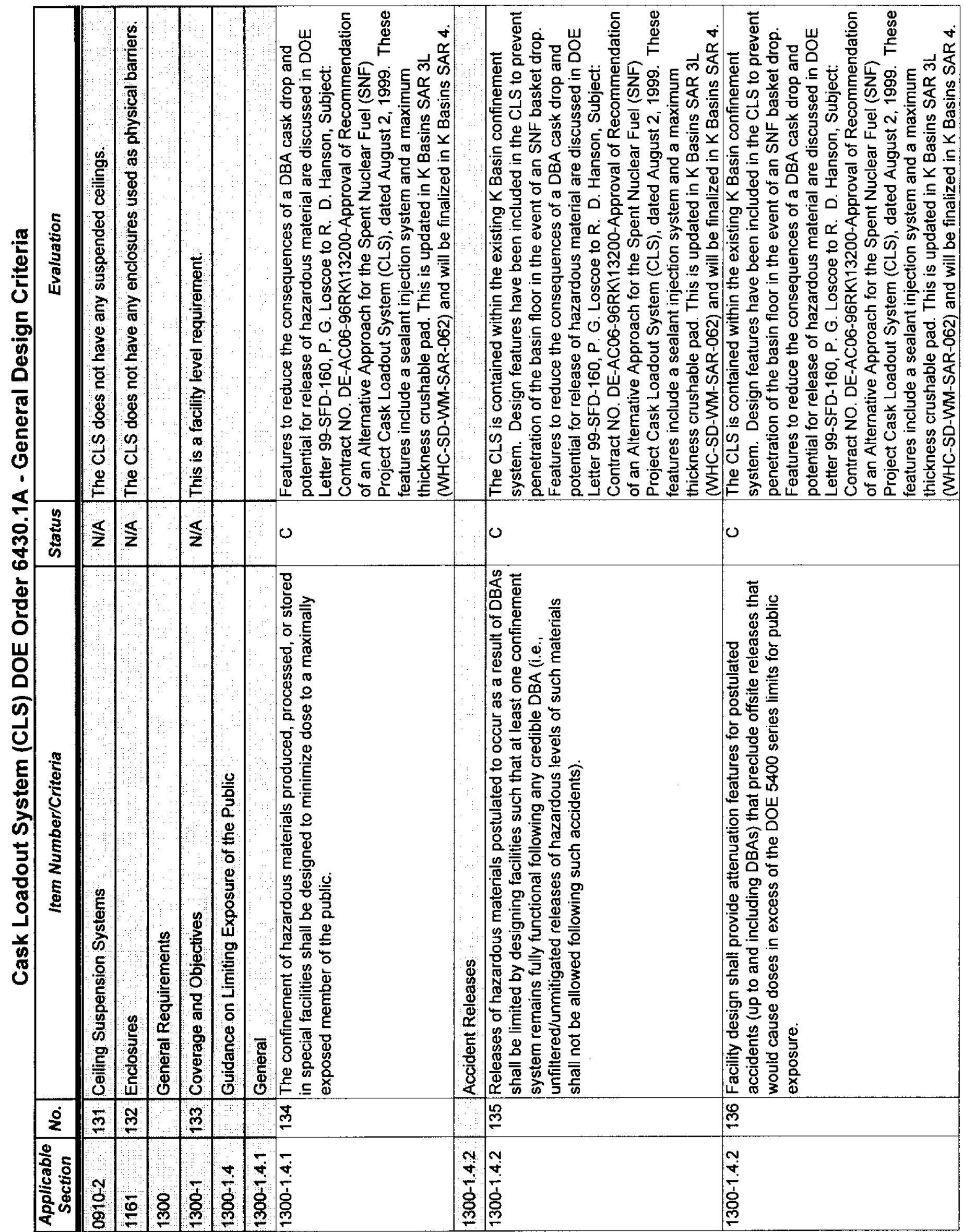


SNF-4775, Rev. 2

Project A.5/A.6

APPENDIX A

\begin{tabular}{|c|c|c|c|c|c|c|c|c|c|c|c|c|}
\hline 总 & & 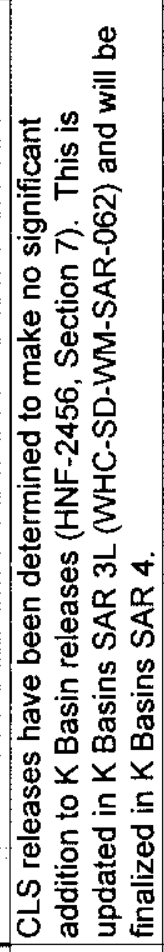 & 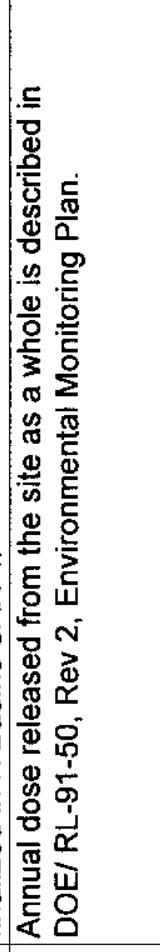 & & 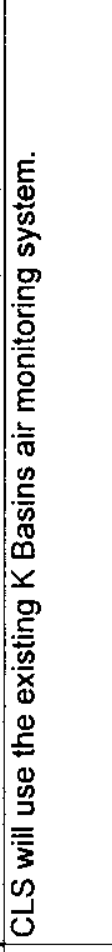 & & 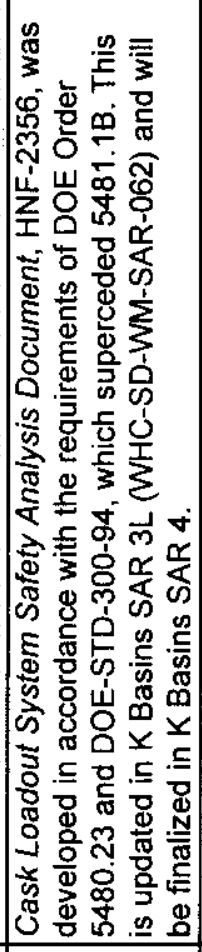 & & & & 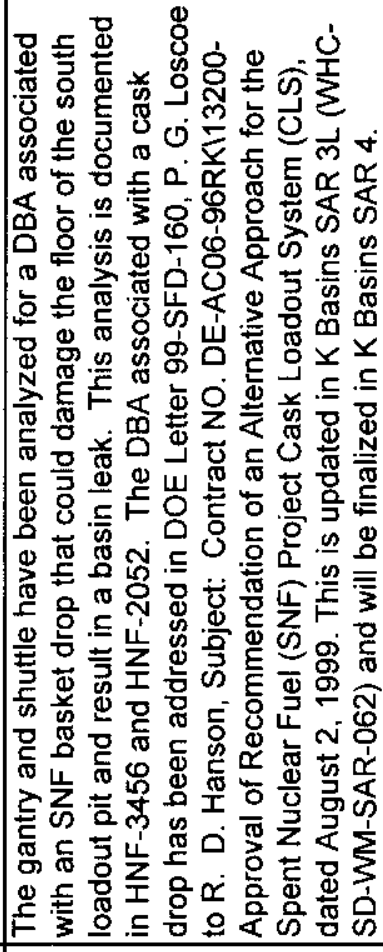 & 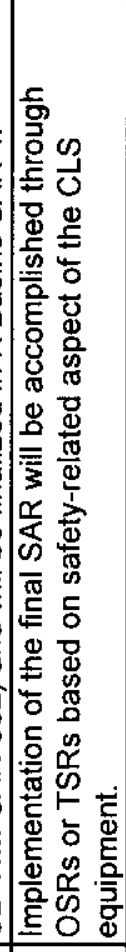 \\
\hline 苞 & & 0 & $\stackrel{\frac{1}{z}}{z}$ & & $\frac{\$}{z}$ & & 0 & & & & 0 & 0 \\
\hline 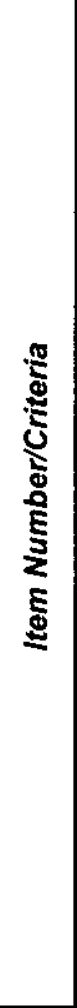 & 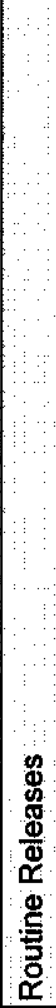 & 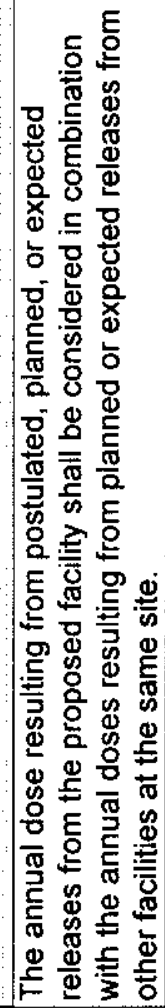 & 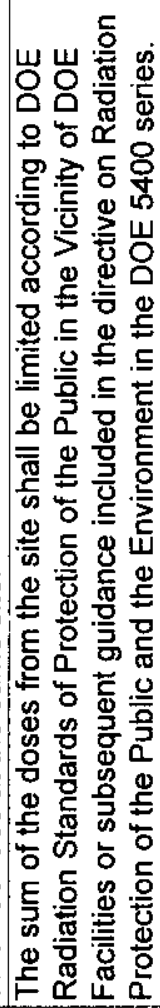 & 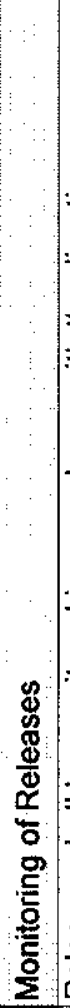 & 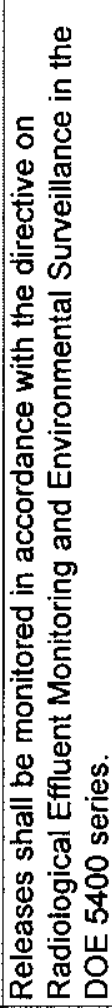 & 变 & 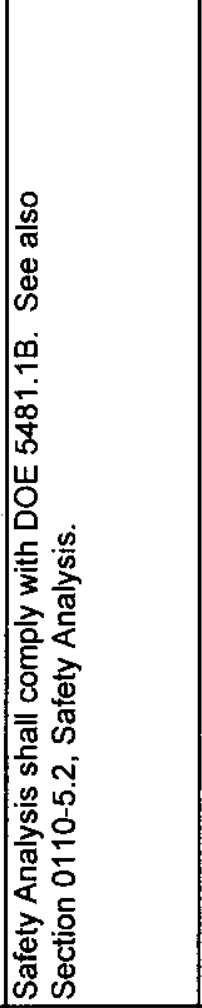 & 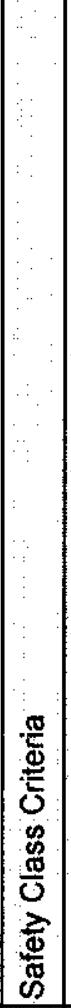 & 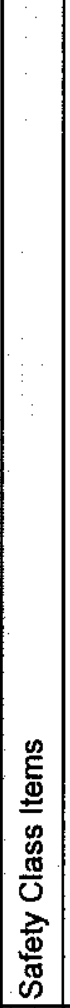 & 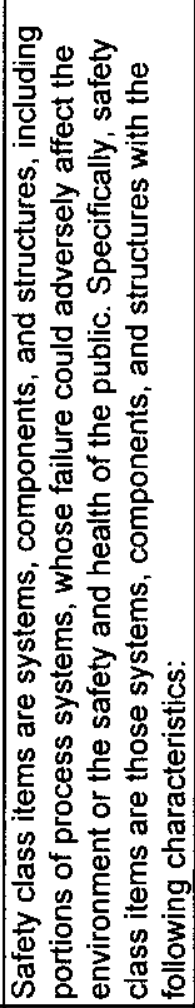 & 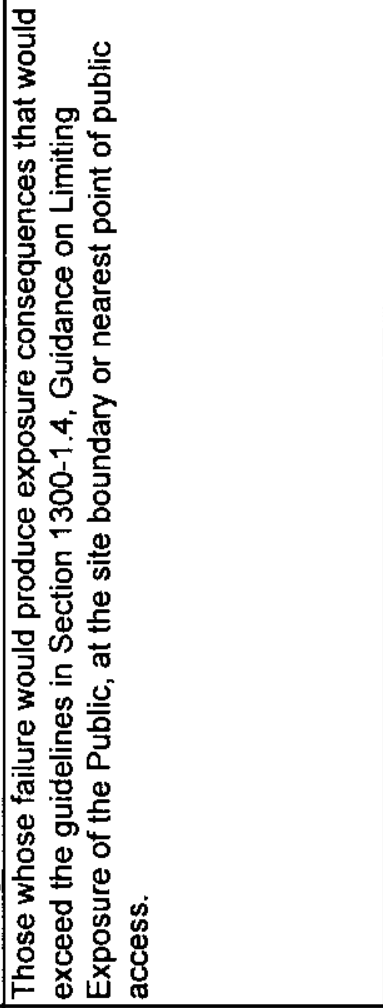 & 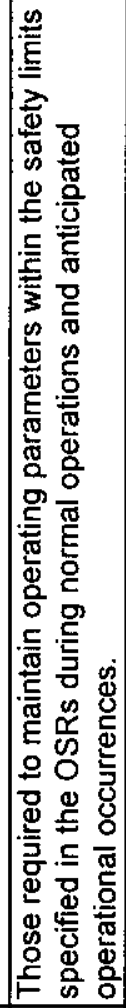 \\
\hline i & & $\stackrel{9}{9}$ & $\stackrel{\infty}{\sim}$ & & gi & & 守 & & & $\bar{z}$ & I & $\dot{*}$ \\
\hline 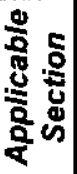 & 尺े & 孚 & $\begin{array}{l}m \\
\dot{y} \\
\dot{\leftrightarrow} \\
\grave{8}\end{array}$ & & & P & $\because-$ & 宫 & $\begin{array}{l}m \\
\dot{8} \\
\bar{p}\end{array}$ & $\stackrel{2}{2}$ & 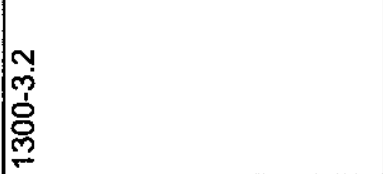 & 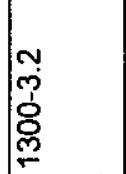 \\
\hline
\end{tabular}




\begin{tabular}{|c|c|c|c|c|c|c|c|}
\hline & 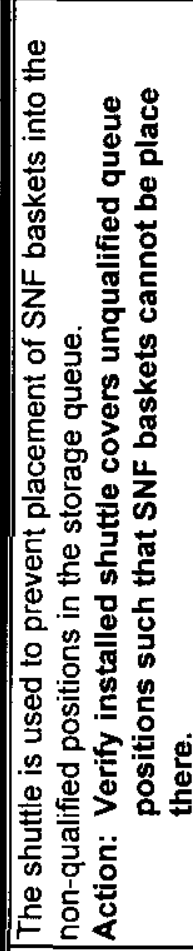 & 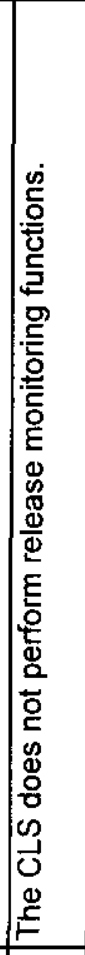 & 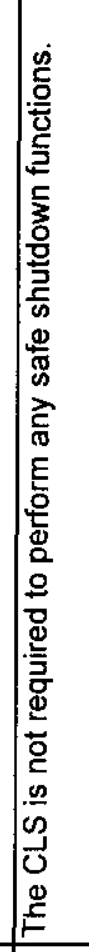 & 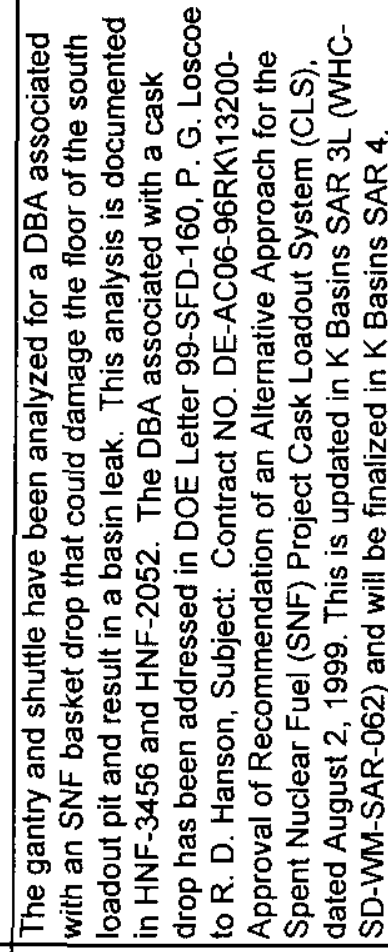 & 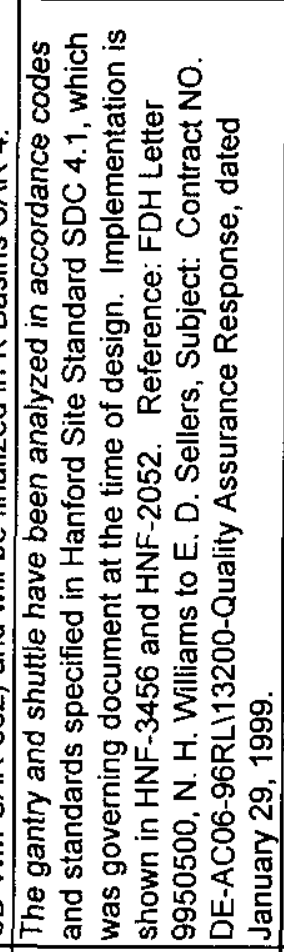 & 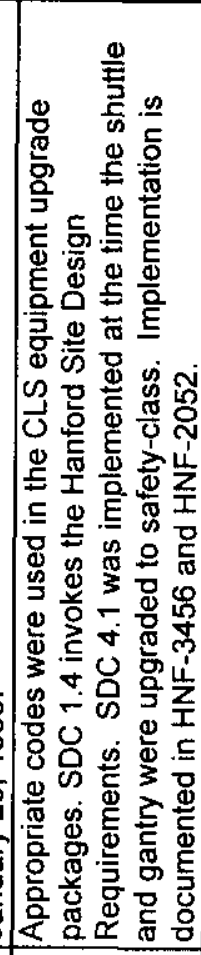 & 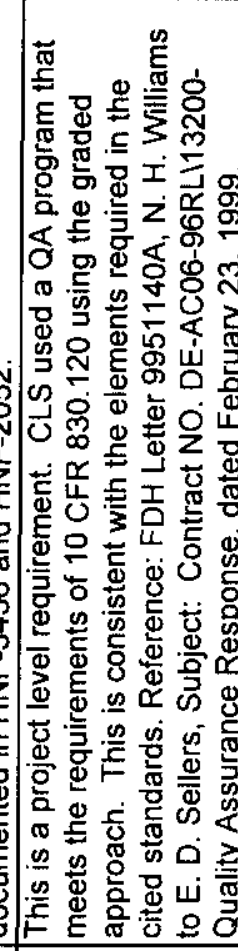 \\
\hline 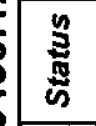 & \begin{tabular}{|l} 
\\
嵅 \\
\end{tabular} & 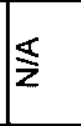 & $\mid \frac{\$}{\mathbf{z}}$ & 0 & 0 & 0 & 0 \\
\hline 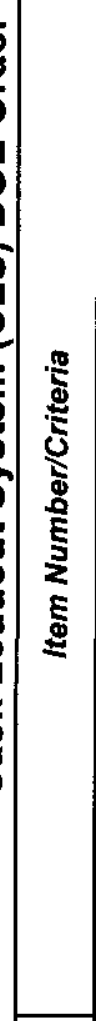 & 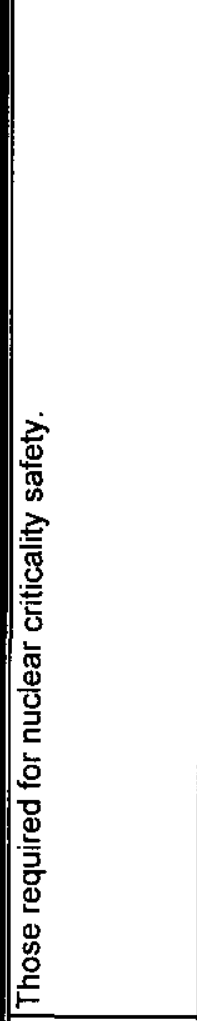 & 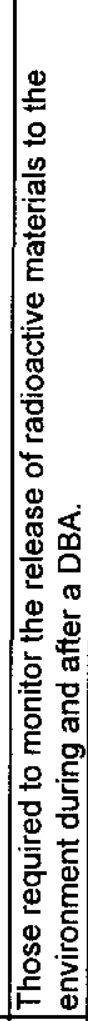 & 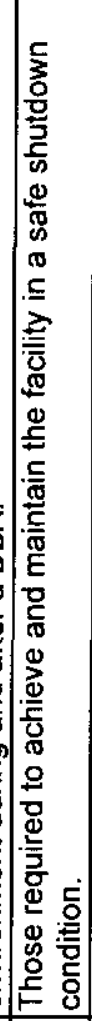 & 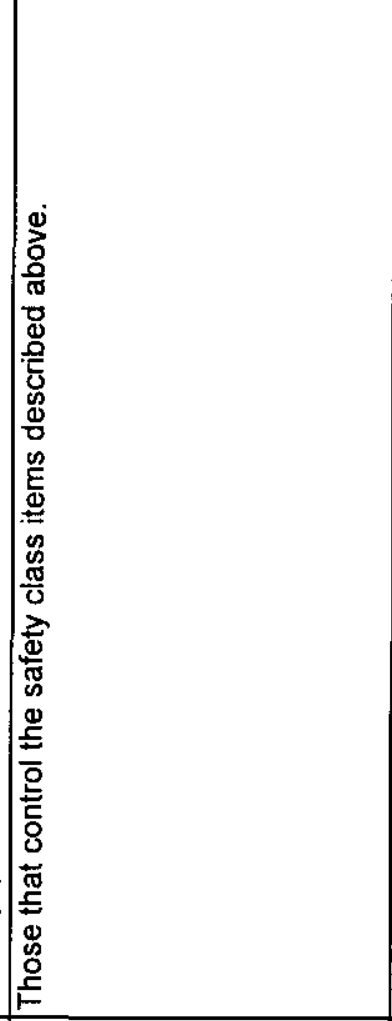 & 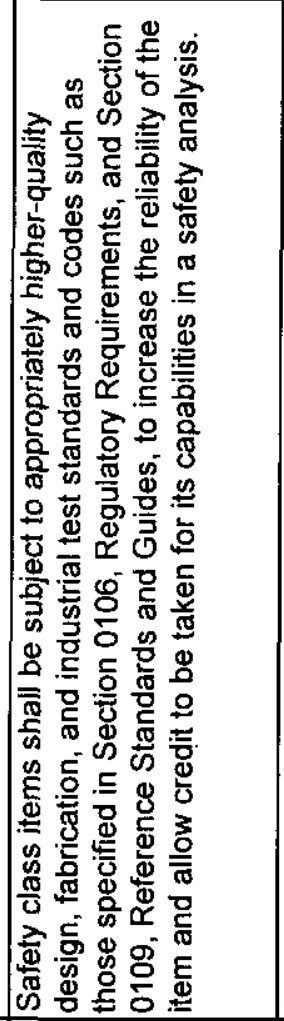 & 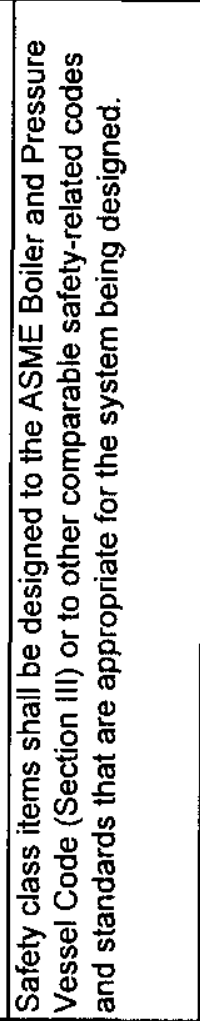 & 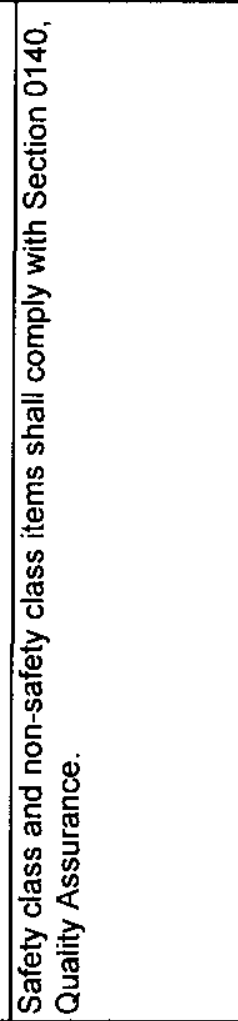 \\
\hline ¿́丶 & J & $\mathscr{q}$ & 果 & $\hat{\xi}$ & 杰 & 守 & 18 \\
\hline $\mid$ & 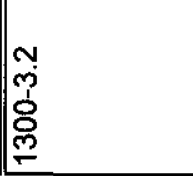 & 帒 & 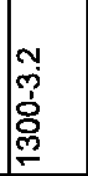 & 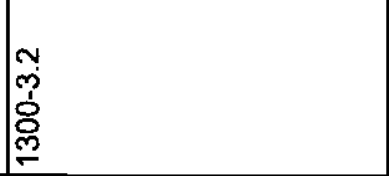 & 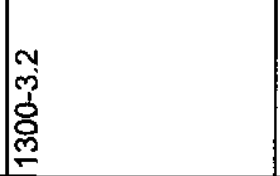 & m & 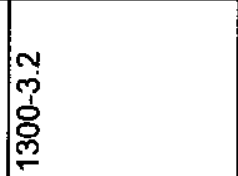 \\
\hline
\end{tabular}


SNF-4775, Rev. 2

Project A.5/A.6

APPENDIX A

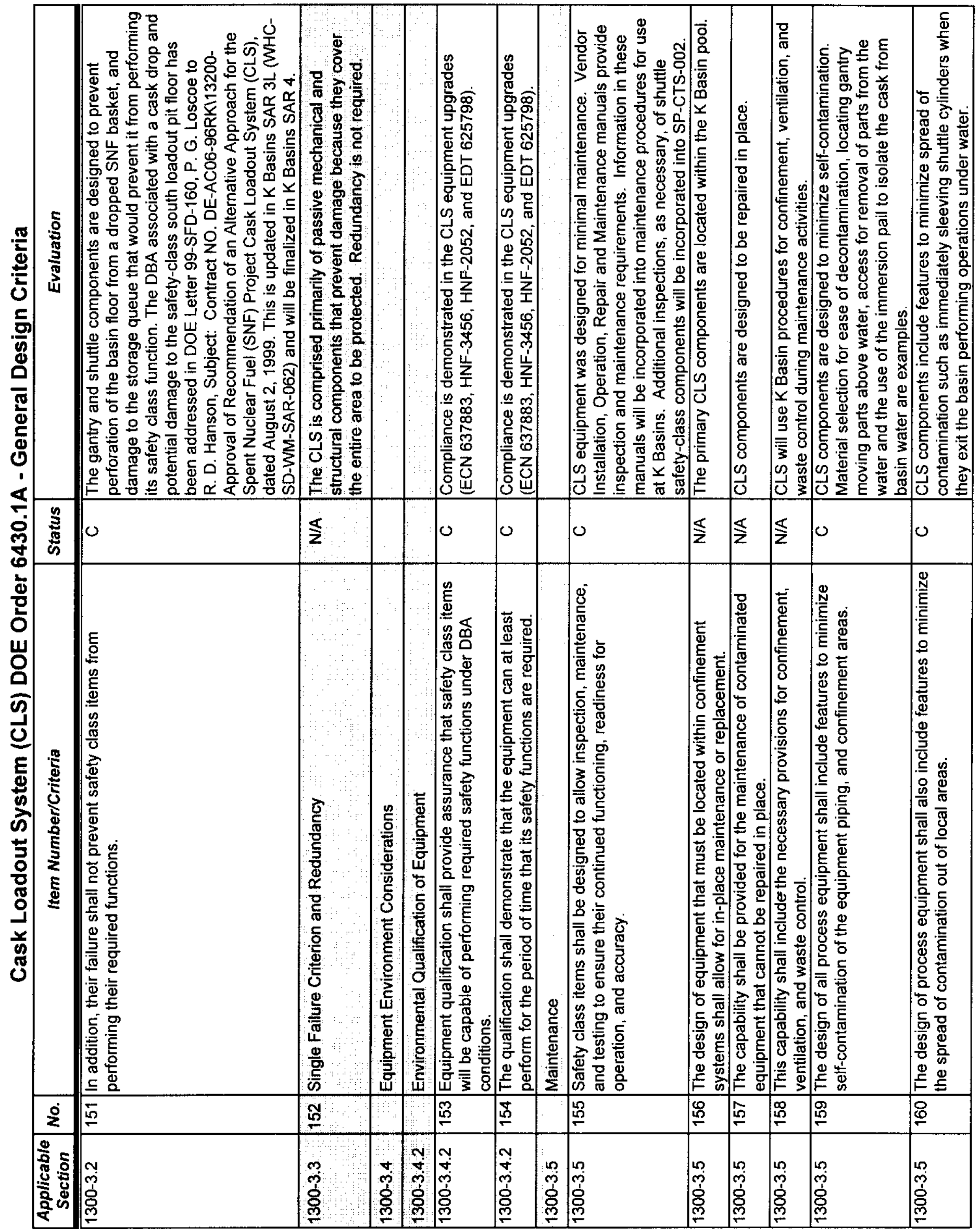




\begin{tabular}{|c|c|c|c|c|c|c|c|c|c|c|c|}
\hline 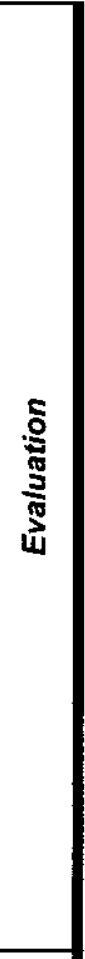 & & 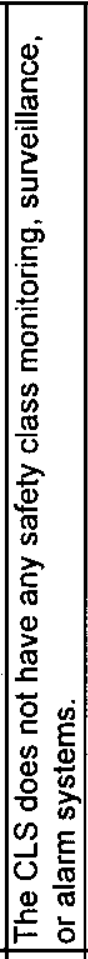 & 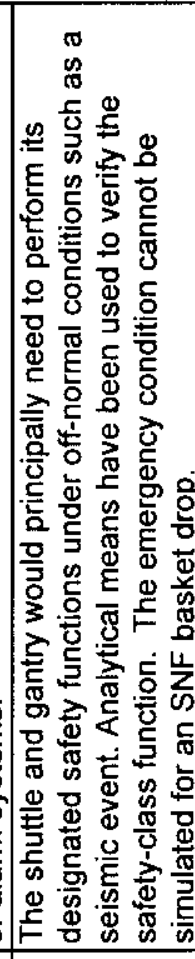 & 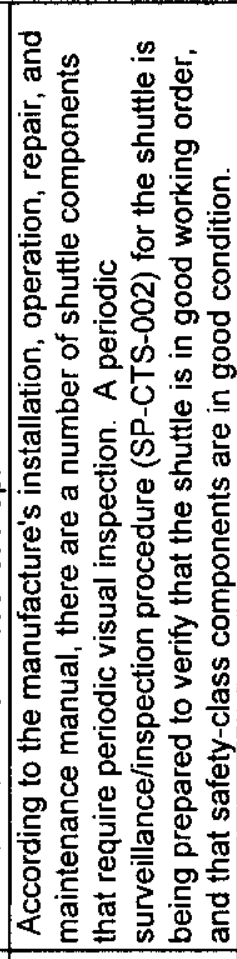 & 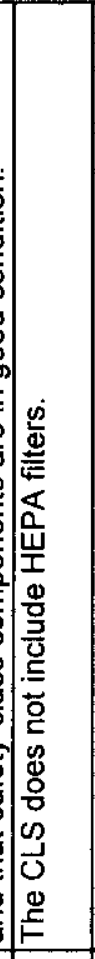 & & 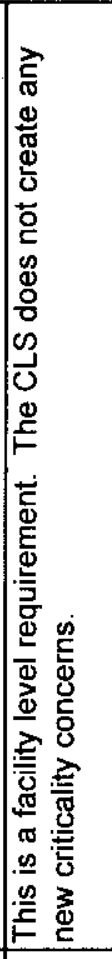 & 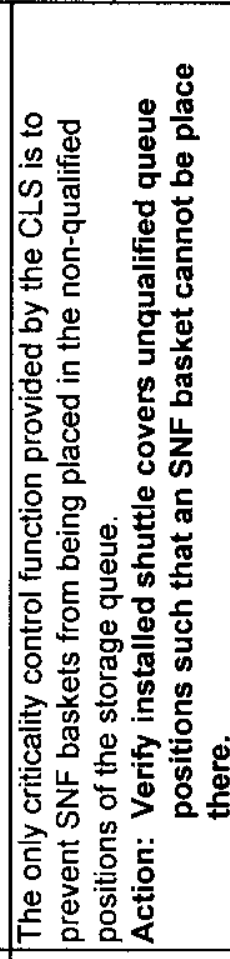 & 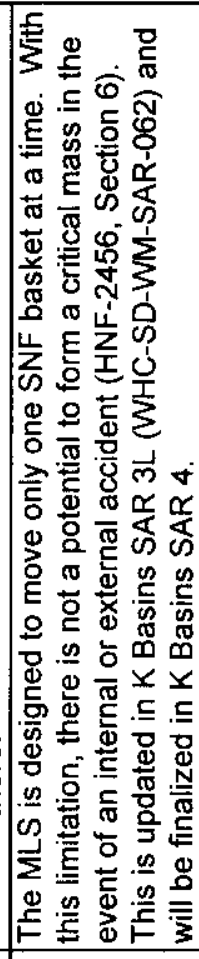 & 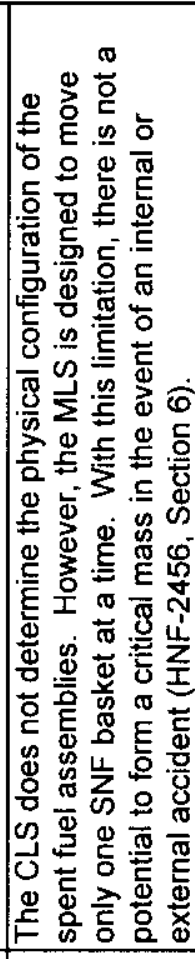 & 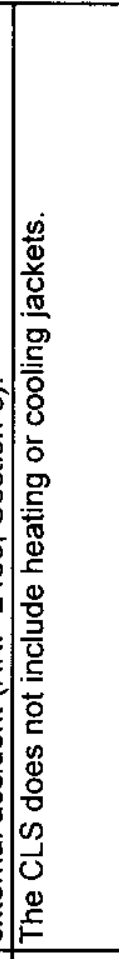 \\
\hline 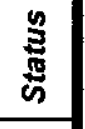 & & 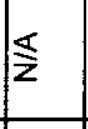 & 0 & 0 & 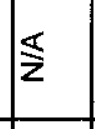 & & $\bar{z}$ & 늠 & 0 & $\bar{z}$ & $\overline{\mathbf{z}}$ \\
\hline 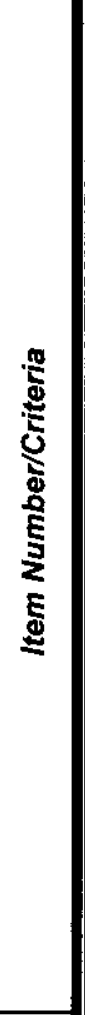 & $\stackrel{8}{5}$ & 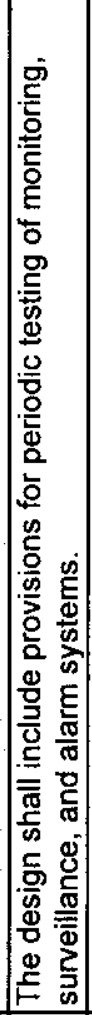 & 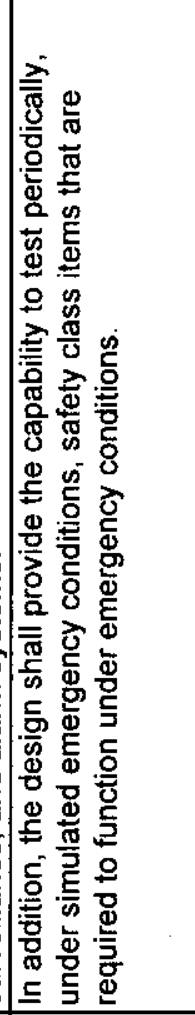 & 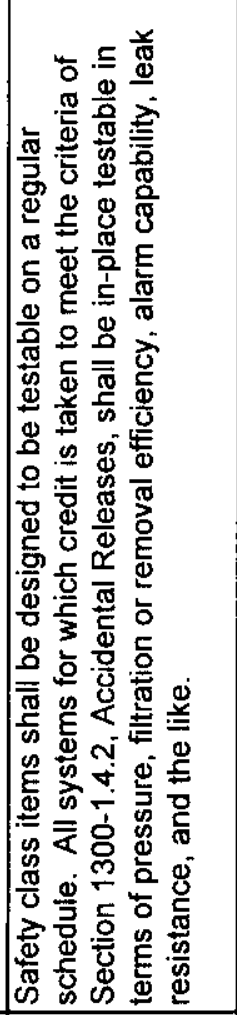 & 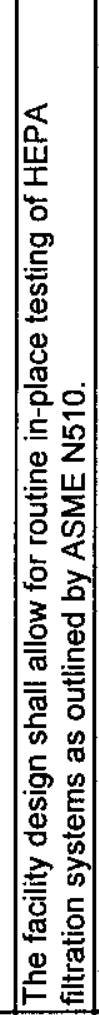 & 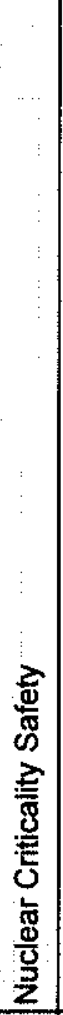 & 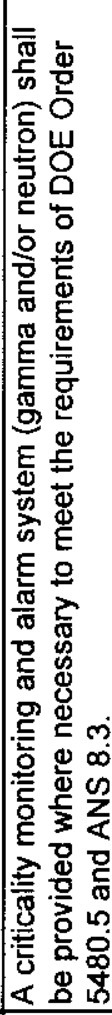 & 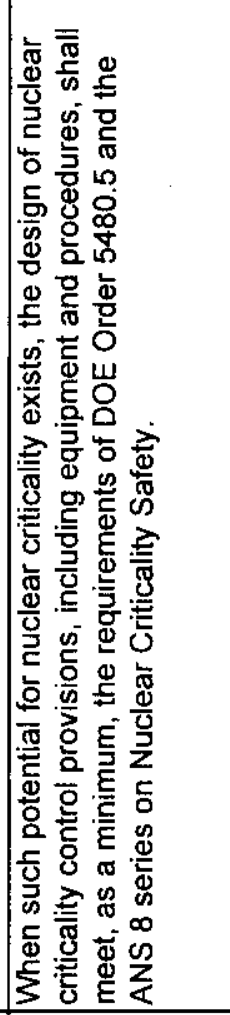 & 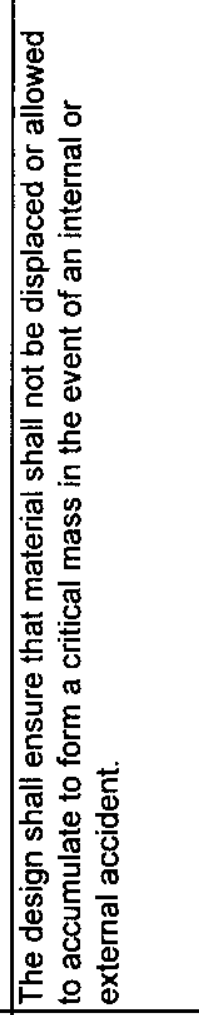 & 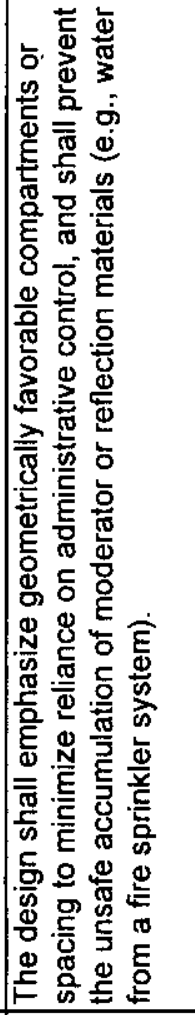 & 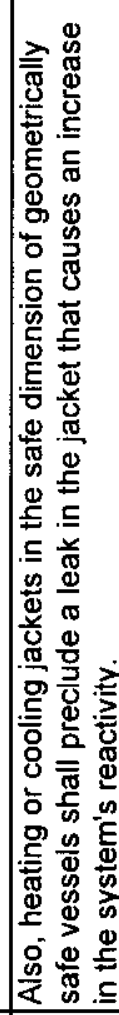 \\
\hline$i^{\circ}$ & & 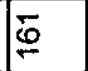 & Ð & $\mathscr{8}$ & $\Phi$ & & 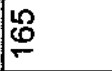 & $\dddot{\mathscr{O}}$ & $\mathscr{O}$ & $\stackrel{\circ}{\varrho}$ & $\underset{6}{\dddot{8}}$ \\
\hline ڤั & ஜ̊. & স্ষ & 占 & $\mathrm{m}$ & 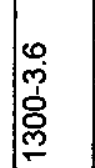 & & $1-$ & & চ্লি & $\stackrel{m}{2}$ & প্ণি \\
\hline
\end{tabular}




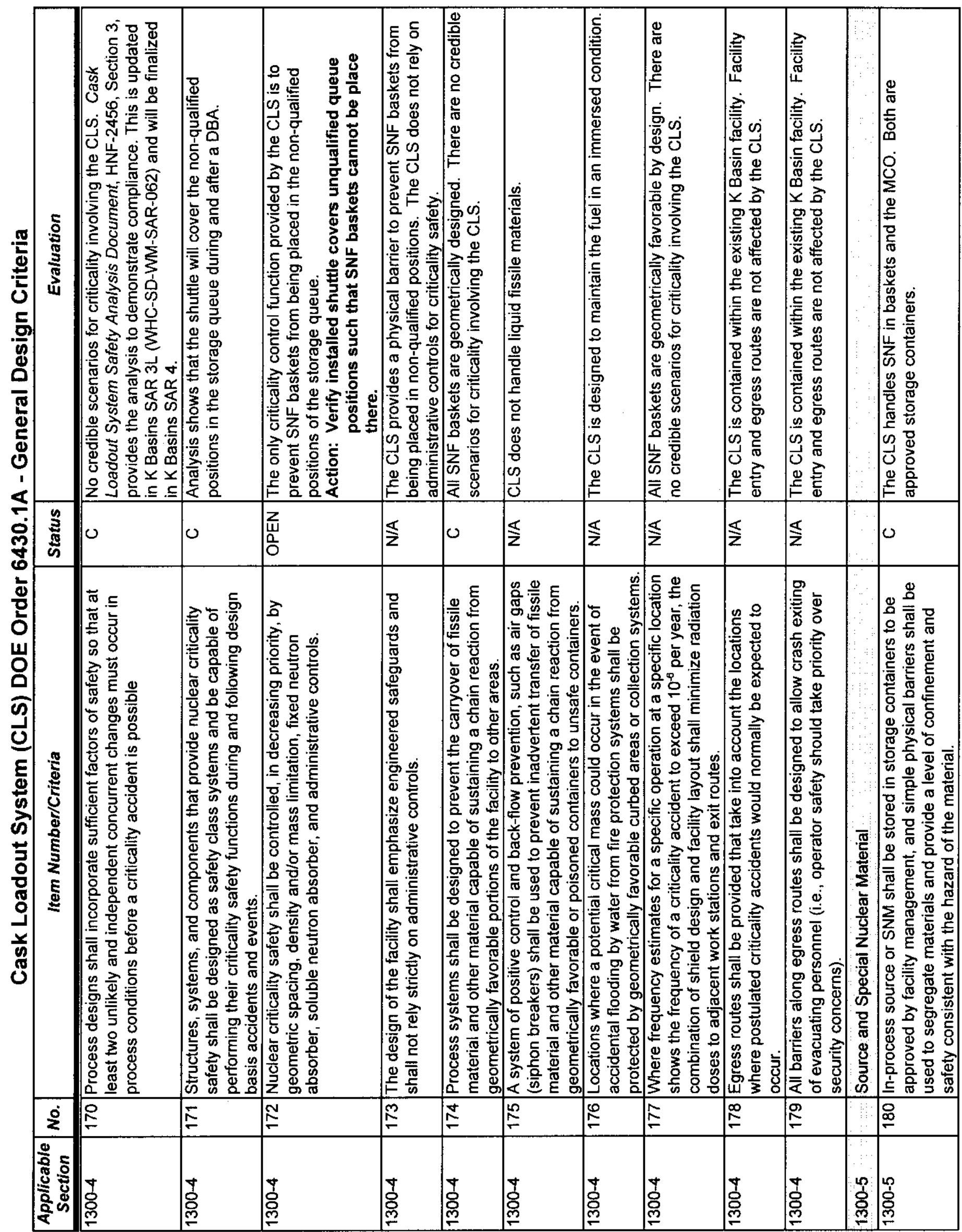


SNF-4775, Rev. 2

Project A.5/A.6

APPENDIX A

\begin{tabular}{|c|c|c|c|c|c|c|c|c|c|c|c|c|c|c|c|c|c|c|c|}
\hline $\mid$ & & & 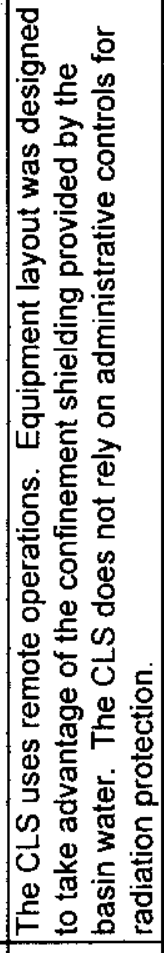 & 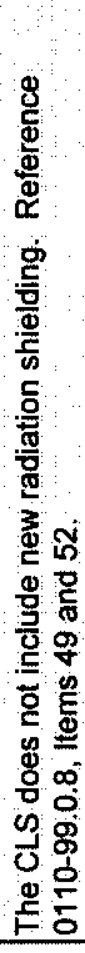 & 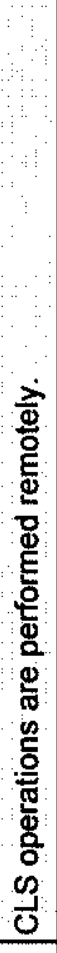 & & 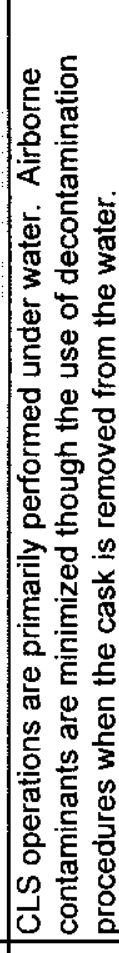 & 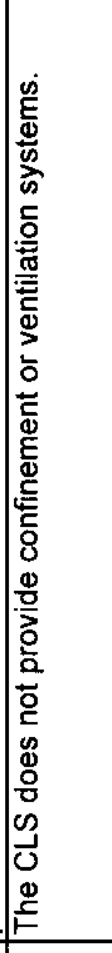 & 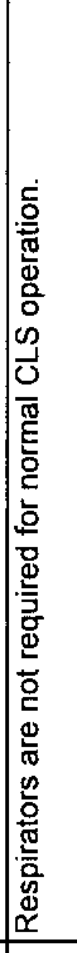 & 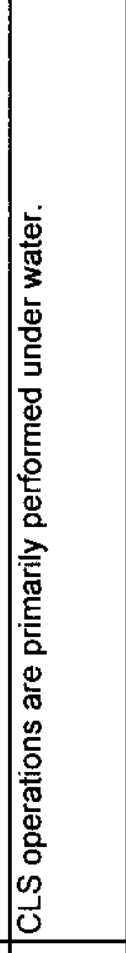 & 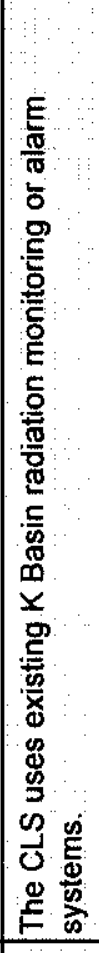 & 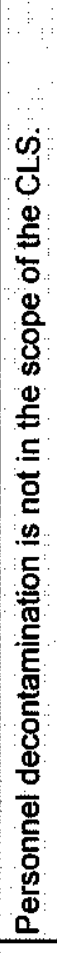 & 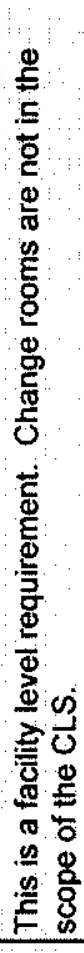 & 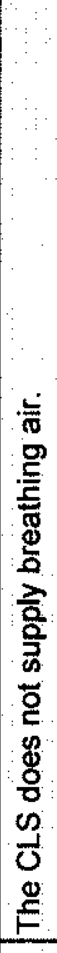 & 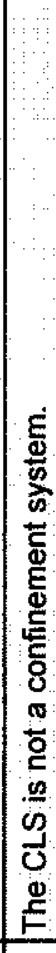 & 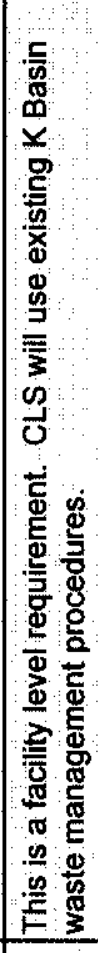 & 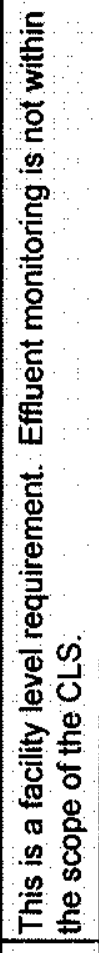 & 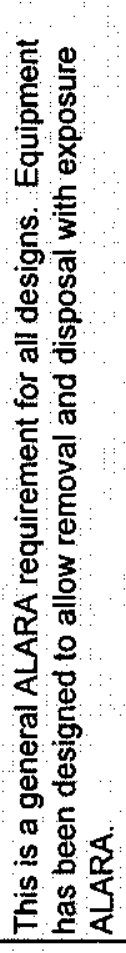 & \\
\hline 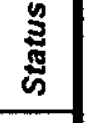 & & & 0 & $\S$ & 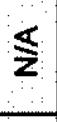 & & 0 & $\frac{\$}{\mathbf{z}}$ & 0 & 0 & $\$$ & 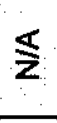 & 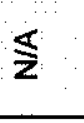 & $\stackrel{\Sigma}{\mathbf{z}}$ & $\frac{5}{z}$ & 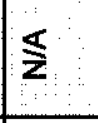 & $z$ & 0 & \\
\hline 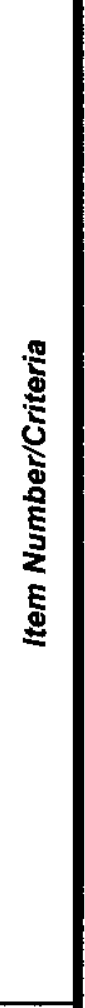 & 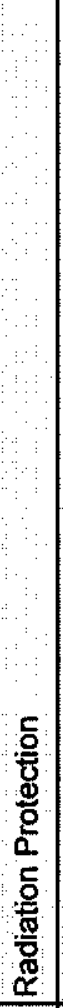 & 푱 & 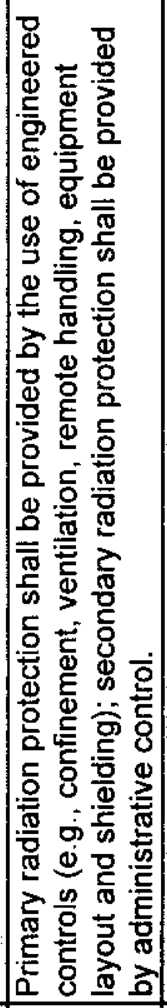 & $\begin{array}{l}5 \\
\frac{5}{5} \\
\mathbf{0} \\
\text { क } \\
\frac{0}{5} \\
\end{array}$ & 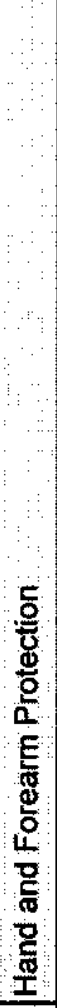 & 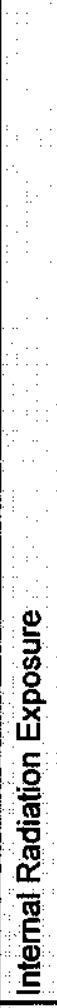 & 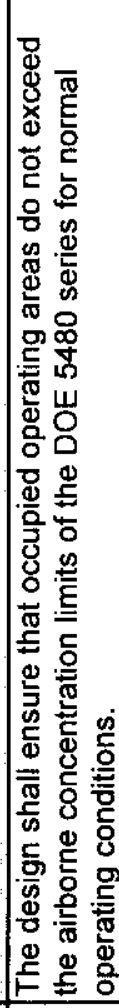 & 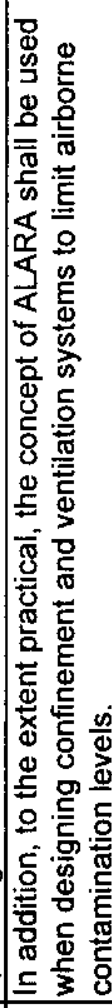 & 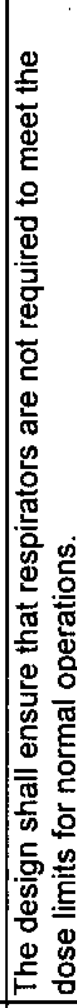 & 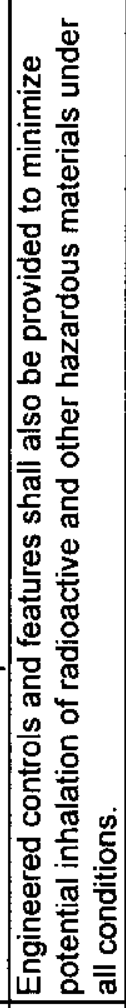 & 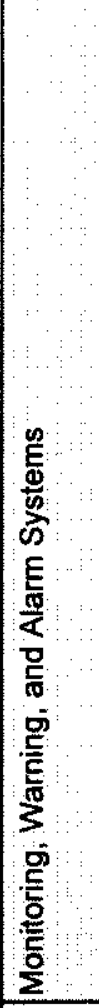 & 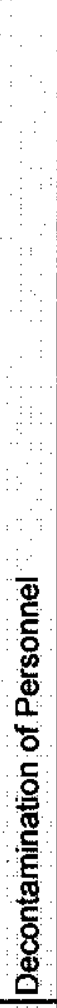 & 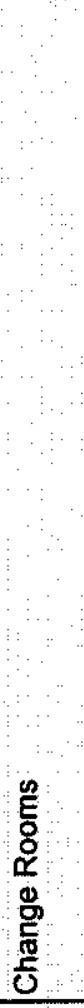 & 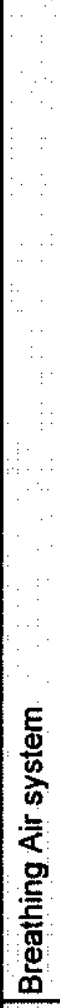 & 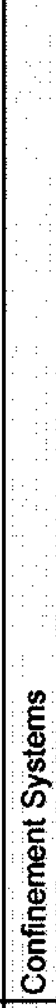 & 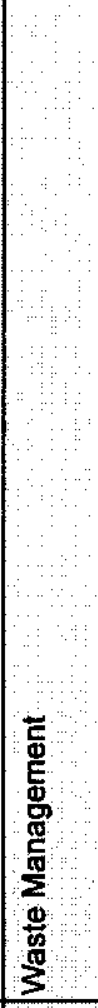 & 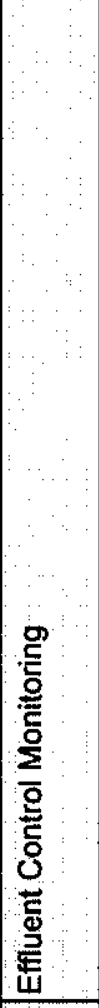 & 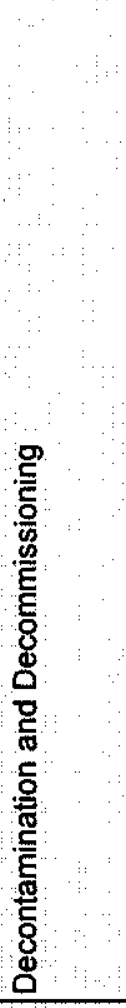 & $\begin{array}{l}8 \\
8 \\
\end{array}$ \\
\hline$\dot{0}$ & & & $\vec{\infty}_{-\infty}$ & న్ర & $\approx$ & & $\underset{\infty}{\infty}$ & $\stackrel{\infty}{\infty}$ & $\dddot{\square}$ & $\stackrel{\infty}{\infty}$ & $\infty$ & $\stackrel{\infty}{\infty}$ & 2 & 19 & ชั & क & $\stackrel{\circ}{\circ}$ & 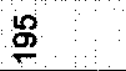 & \\
\hline 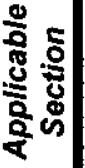 & \& & 영 & 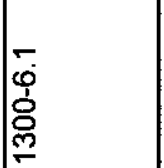 & @્ & ஜ̊ & 总 & 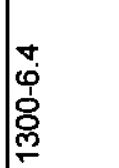 & - & 卤 & 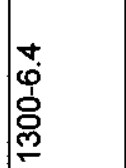 & \% & @̊ & 잉 & ô & $\begin{array}{l}\text { ô } \\
\text { ळ్ }\end{array}$ & ర్ల & 户् & 8 & $=$ \\
\hline
\end{tabular}


SNF-4775, Rev. 2

Project A.5/A.6

APPENDIX A

\begin{tabular}{|c|c|c|c|c|c|c|c|c|c|c|c|c|}
\hline 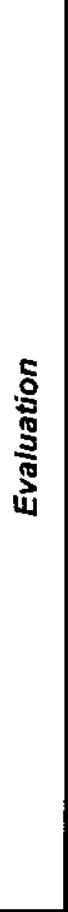 & 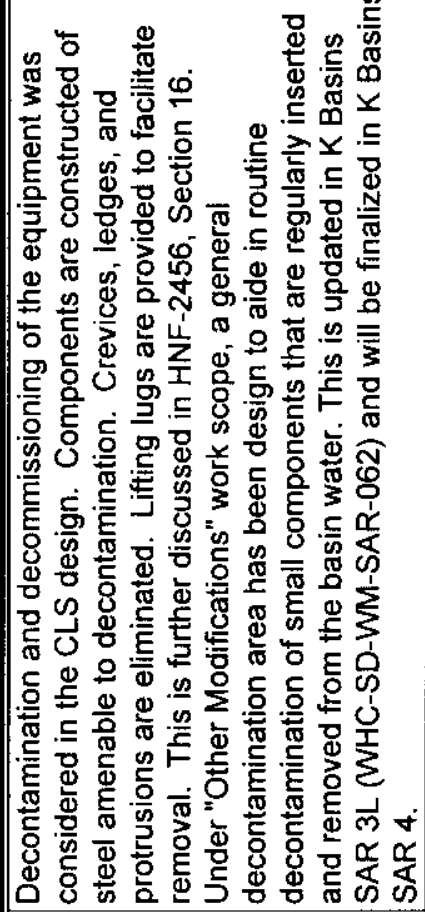 & 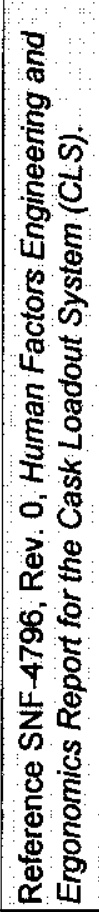 & & 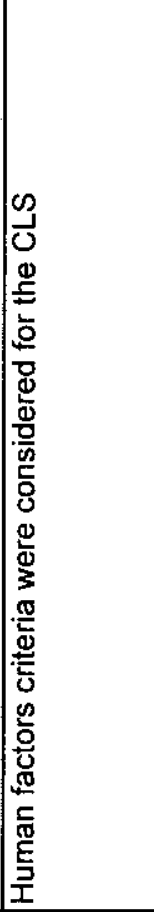 & & 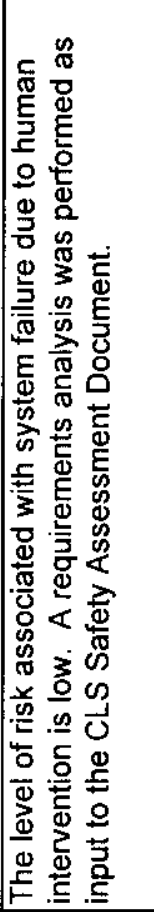 & 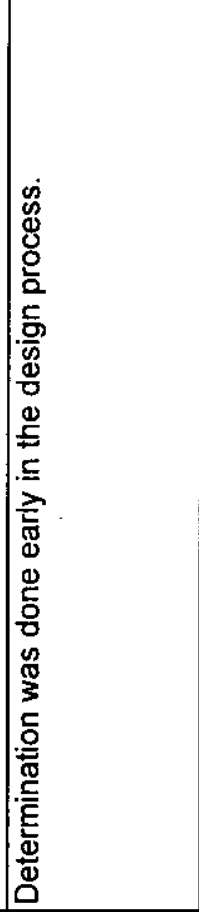 & 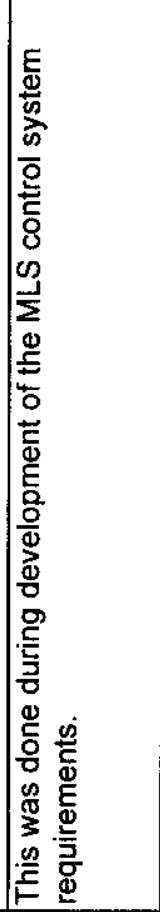 & & 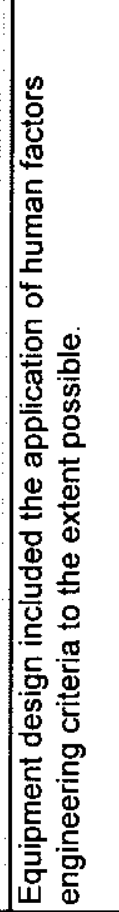 & & \\
\hline 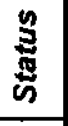 & 0 & & & 0 & & 0 & 0 & 0 & & 0 & & \\
\hline 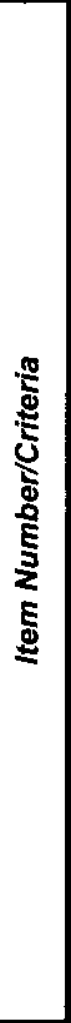 & 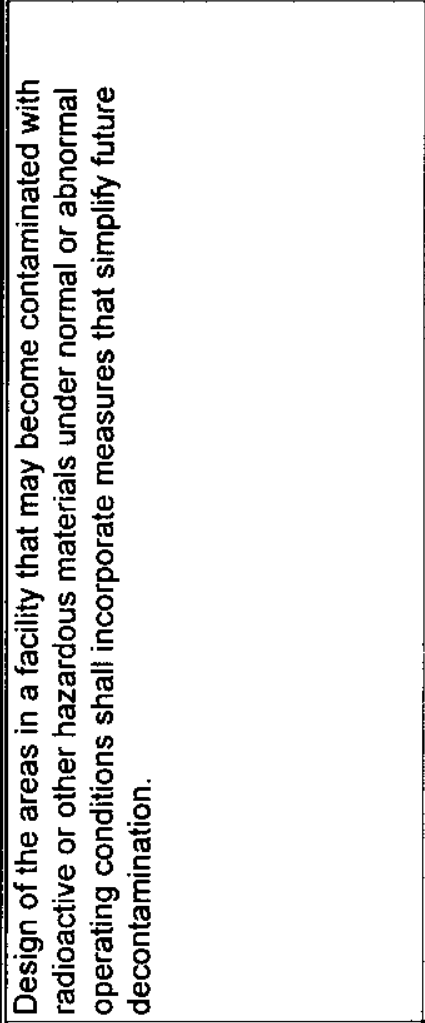 & 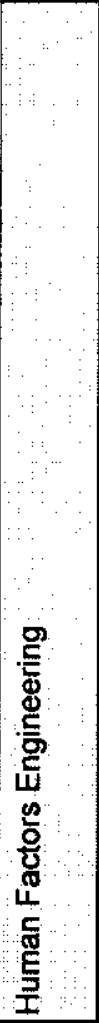 & 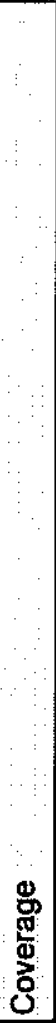 & 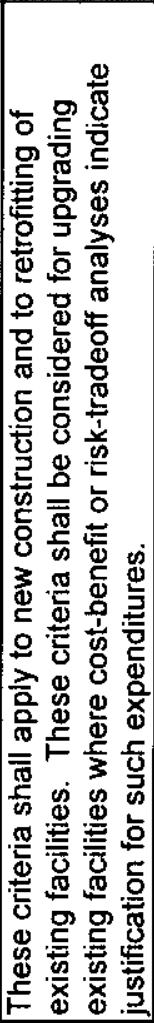 & 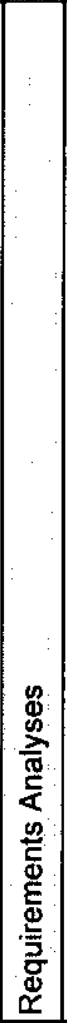 & 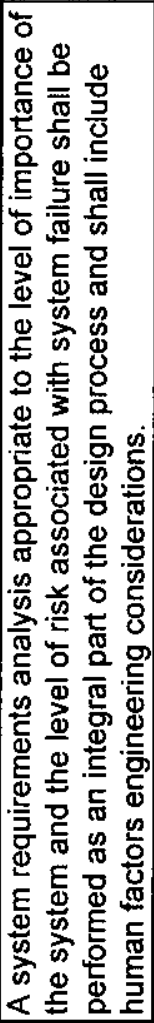 & 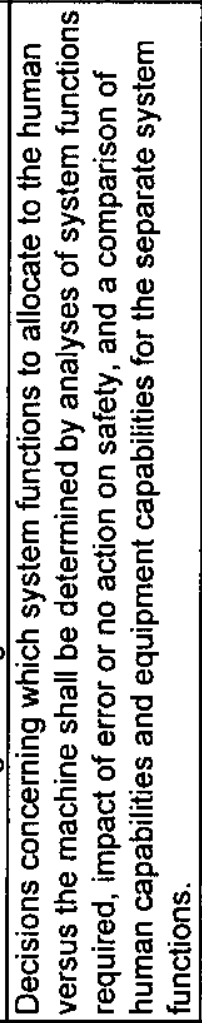 & 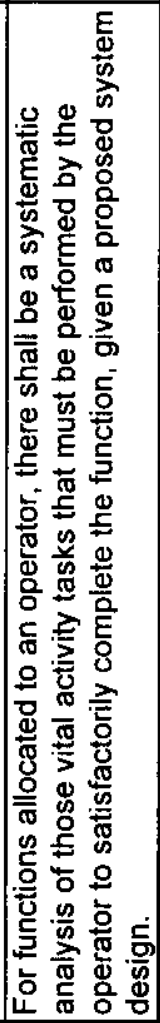 & 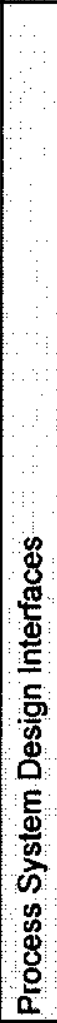 & 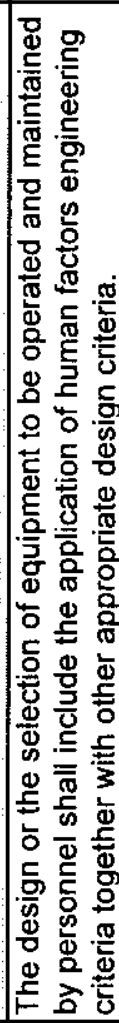 & 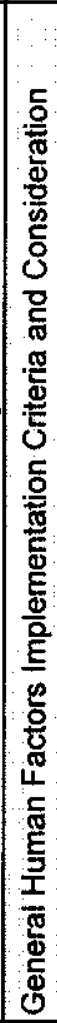 & $\begin{array}{l}\overline{0} \\
\overline{0} \\
\overline{0}\end{array}$ \\
\hline$i^{\circ}$ & $\mathscr{9}$ & & & $\stackrel{5}{2}$ & & $\stackrel{\$}{\stackrel{9}{\circ}}$ & \% & 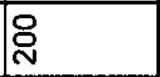 & & I్ & & \\
\hline 吕 & $\check{E}$ & $\underset{్}{\stackrel{N}{\sigma}}$ & సָ & 욤 & & 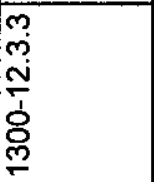 & 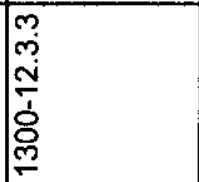 & 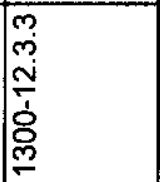 & 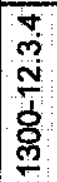 & 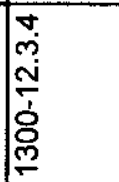 & 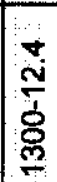 & 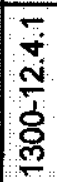 \\
\hline
\end{tabular}


SNF-4775, Rev. 2

Project A.5/A.6

APPENDIX A

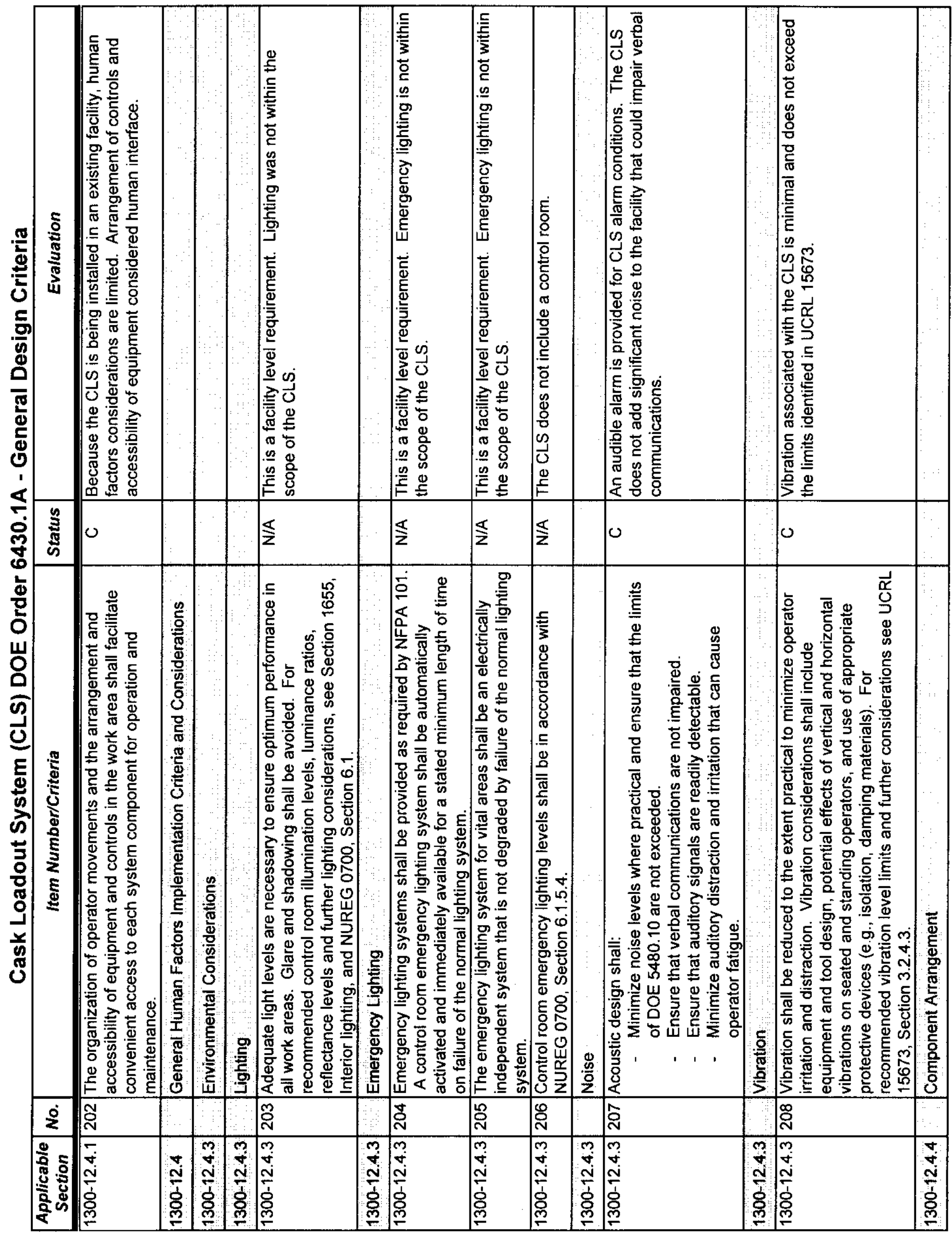


SNF-4775, Rev. 2

Project A.5/A.6

APPENDIX A

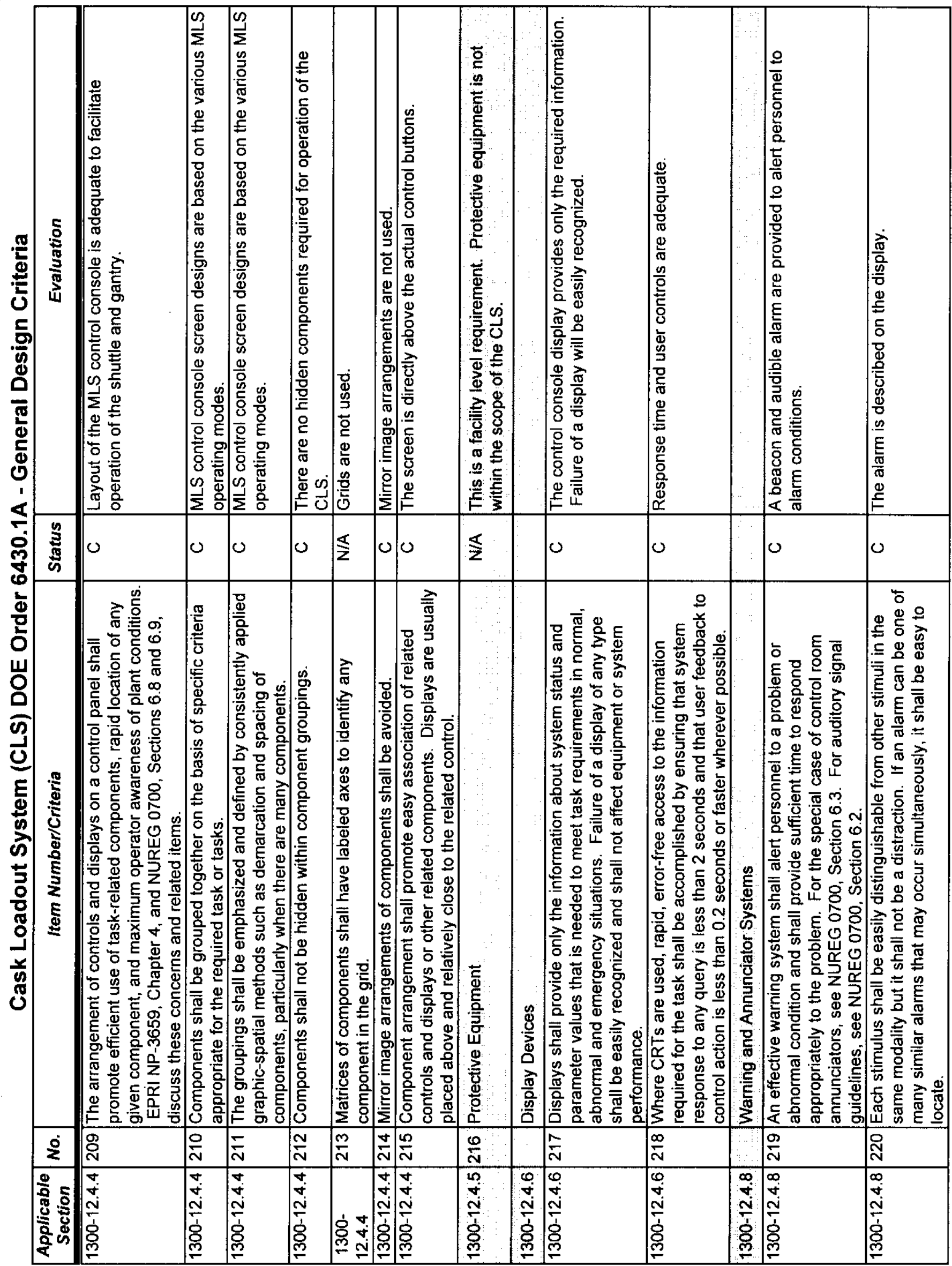


SNF-4775, Rev. 2

Project A.5/A.6

APPENDIX A

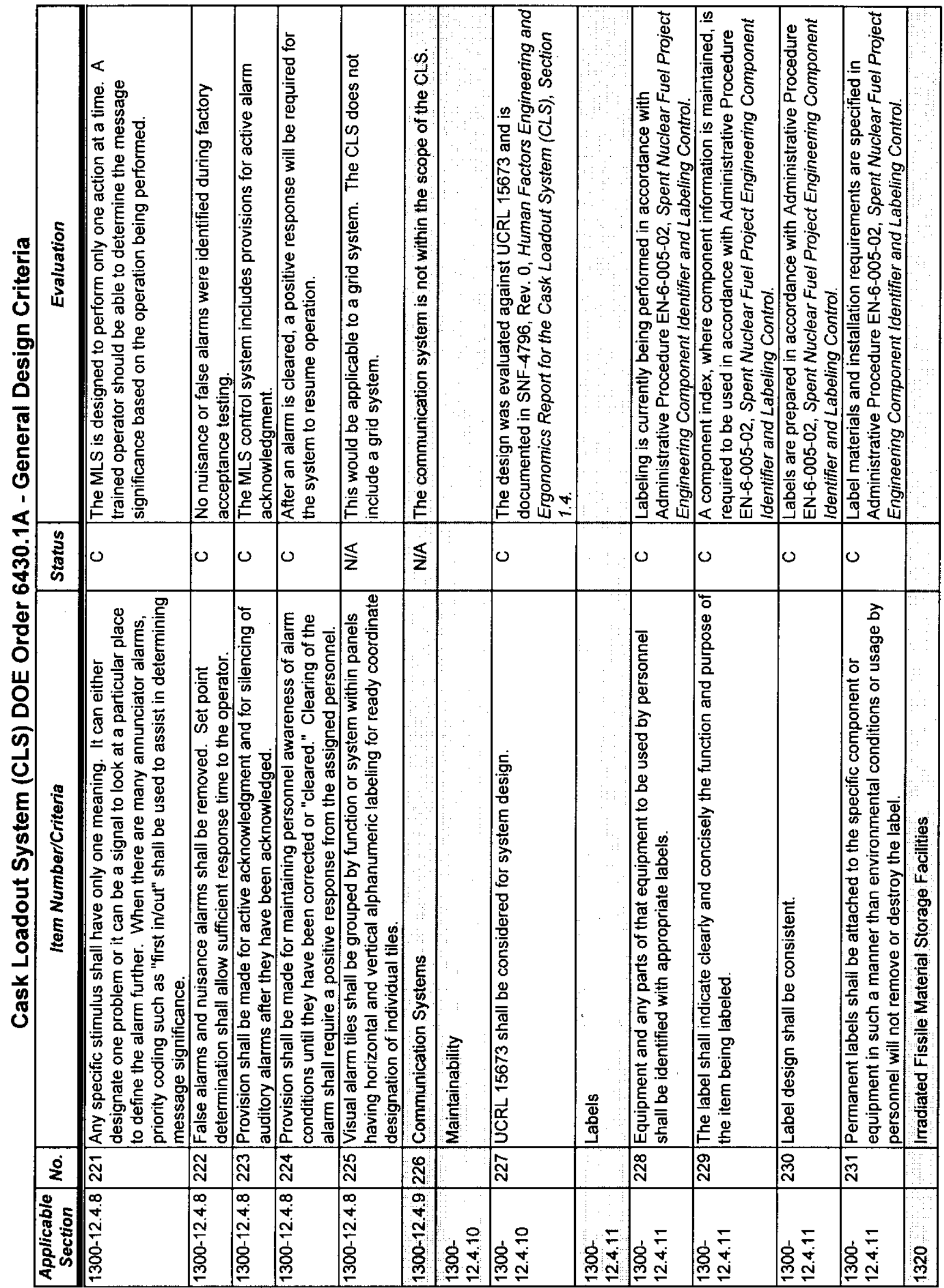


SNF-4775, Rev. 2

Project A.5/A.6

APPENDIX A

\begin{tabular}{|c|c|c|c|c|c|c|c|c|c|c|c|c|c|c|c|c|c|}
\hline 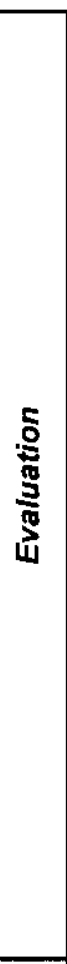 & 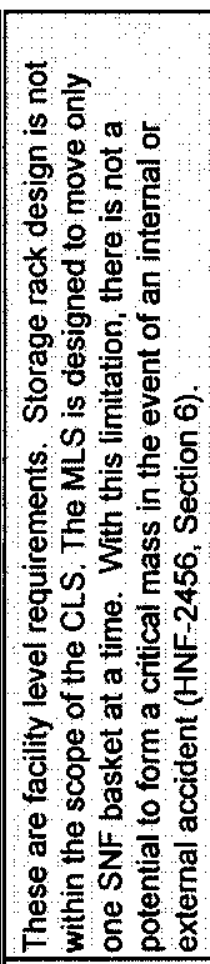 & 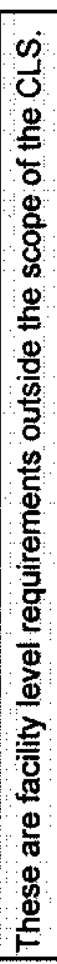 & 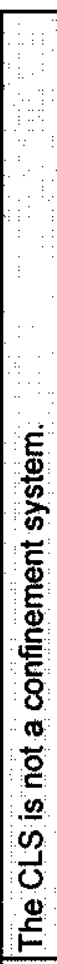 & 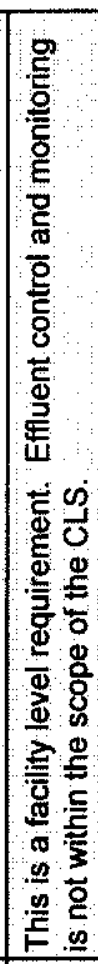 & 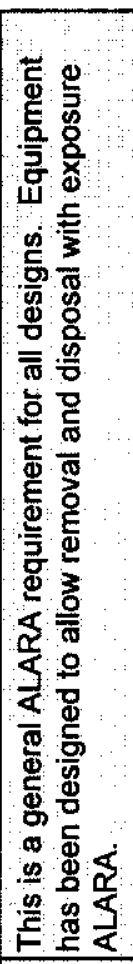 & 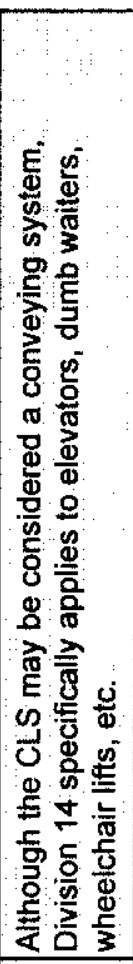 & 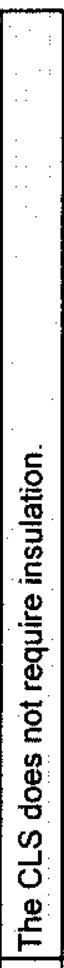 & 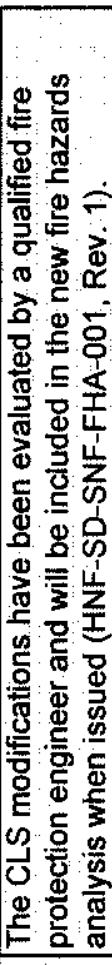 & & 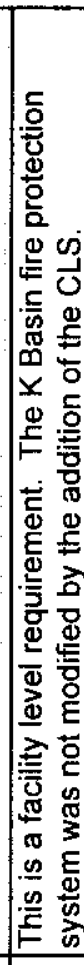 & & & 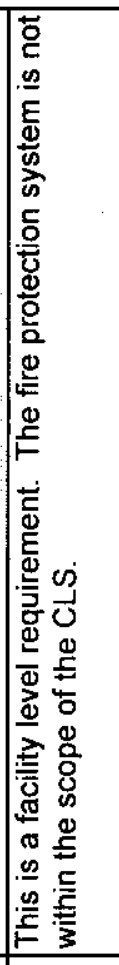 & & 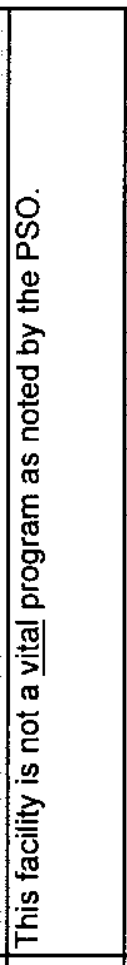 & 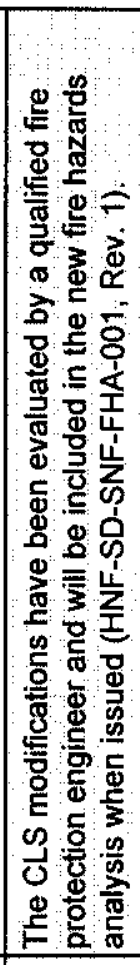 & \\
\hline 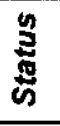 & 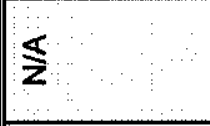 & $\underset{\mathbf{z}}{\mathbf{s}}$ & $\$$ & $\stackrel{\pi}{z}$ & 10 & 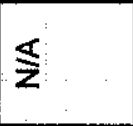 & $\frac{\aleph}{\mathbf{z}}$ & 0 & & $\mid \frac{\$}{z}$ & & & $\frac{\$}{z}$ & & $\mid \frac{\varsigma}{z}$ & & \\
\hline 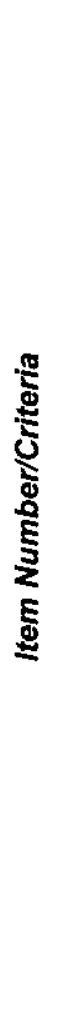 & 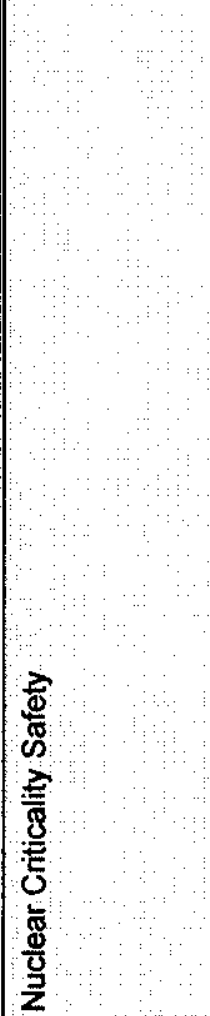 & 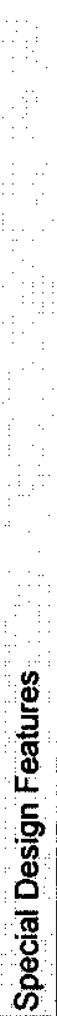 & 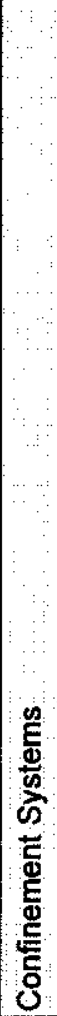 & 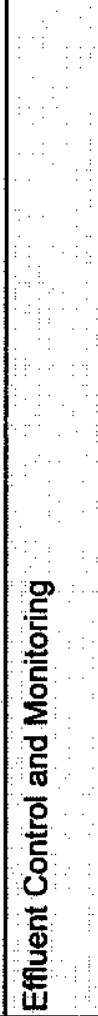 & 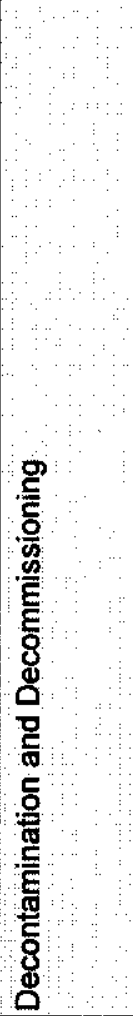 & 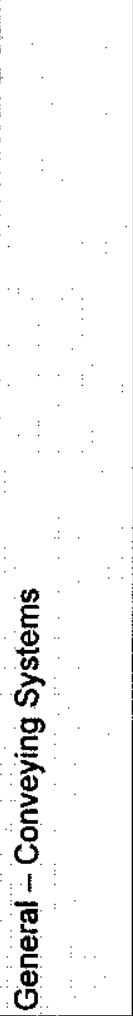 & 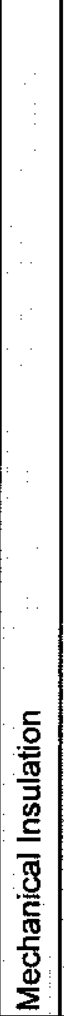 & 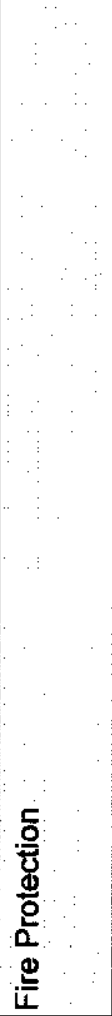 & $\frac{}{\frac{\pi}{2}}$ & 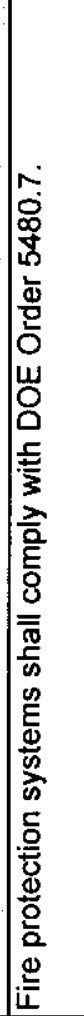 & 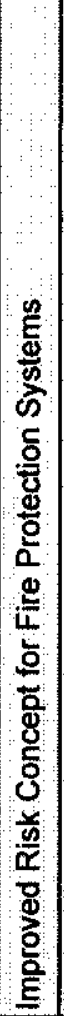 & $\frac{\pi}{\mathbb{\sigma}}$ & 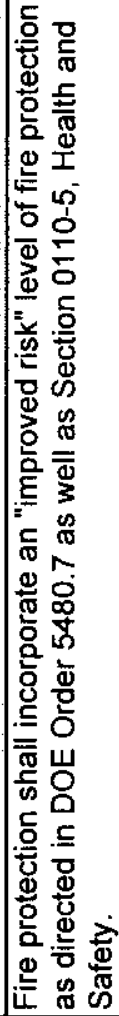 & 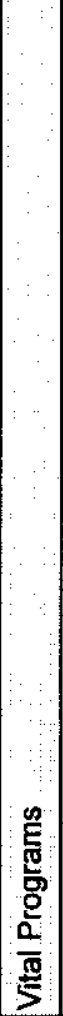 & 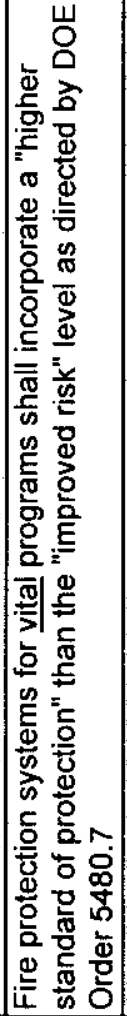 & 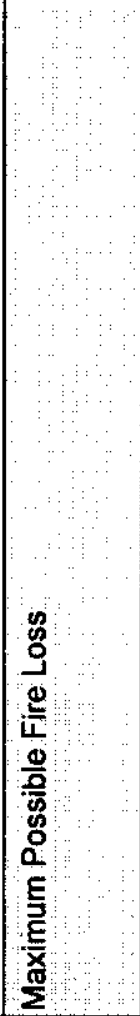 & $\begin{array}{l}\bar{\pi} \\
\overline{4} \\
0\end{array}$ \\
\hline i & బ్ల్ & ๙ & స్ & N & ల్ల & నิ & งి & & & 尺్ల్ల & & & I & & İ & & \\
\hline 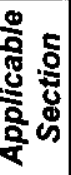 & ग्र & ণ్ర్ల & ్․ & कू్ & 요 & $\stackrel{9}{+}$ & $\stackrel{\mathbb{N}}{\stackrel{2}{2}}$ & గ్ల్ & 윰 & 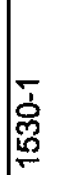 & గ్రి & तु & 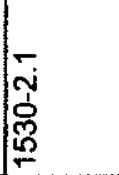 & तें & 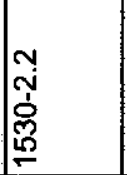 & ते & ஸे \\
\hline
\end{tabular}


SNF-4775, Rev. 2

Project A.5/A.6

APPENDIX A

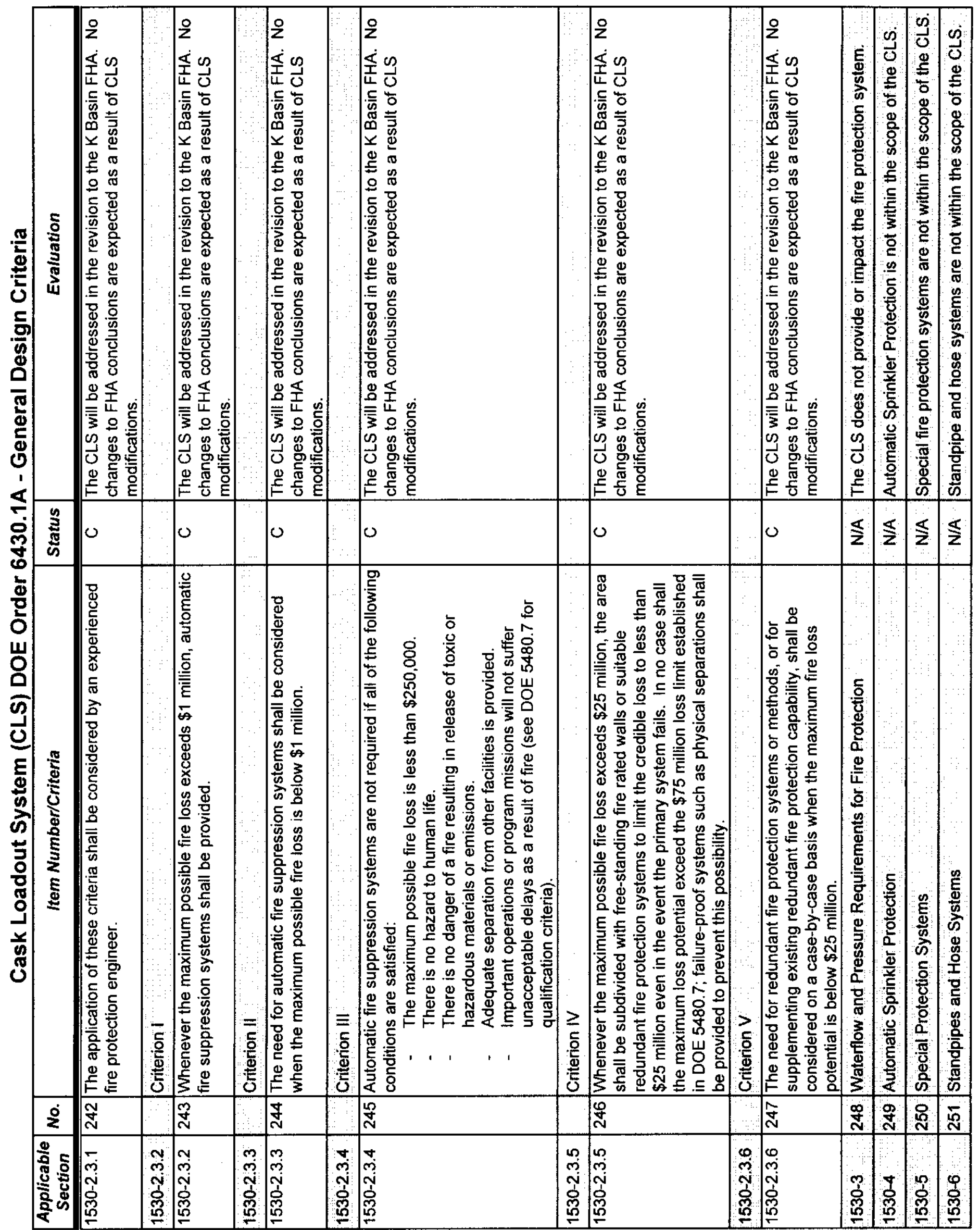




\begin{tabular}{|c|c|c|c|c|c|c|c|c|c|c|c|c|c|c|c|}
\hline 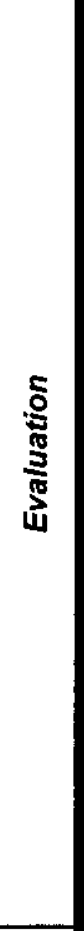 & 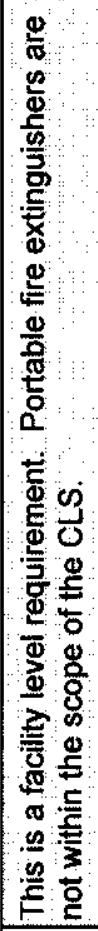 & 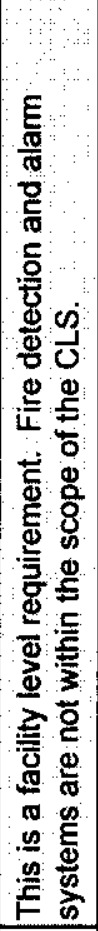 & 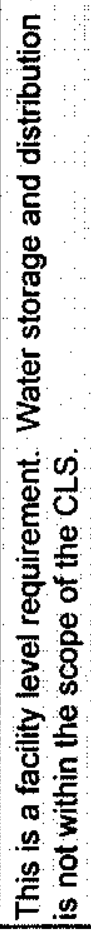 & & & 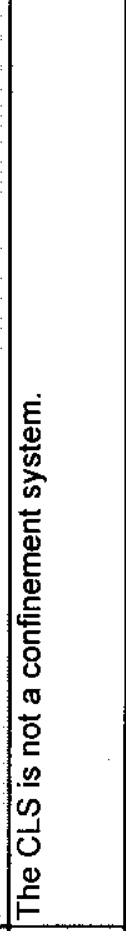 & 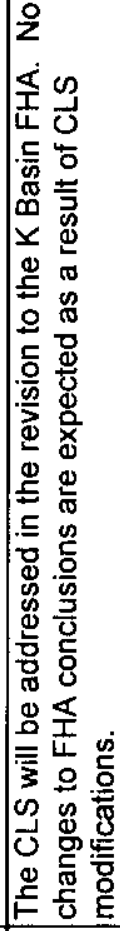 & 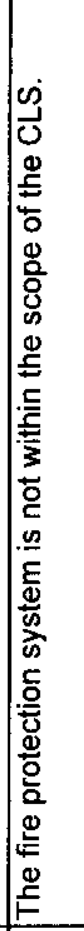 & 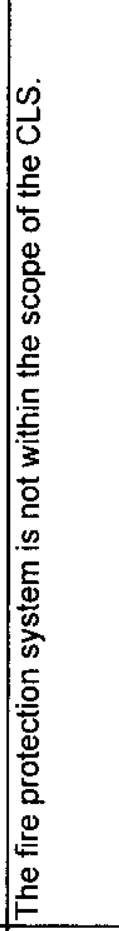 & 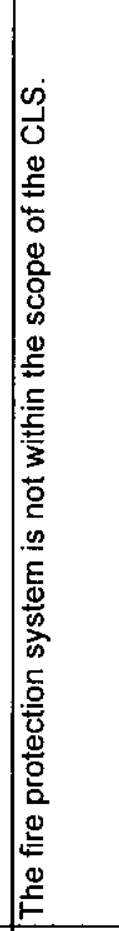 & 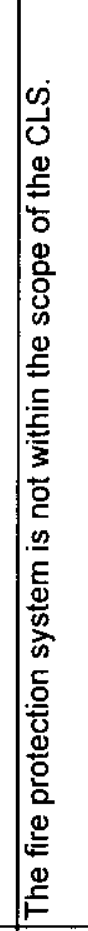 & 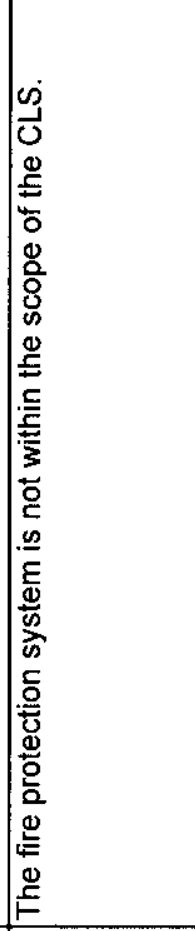 & 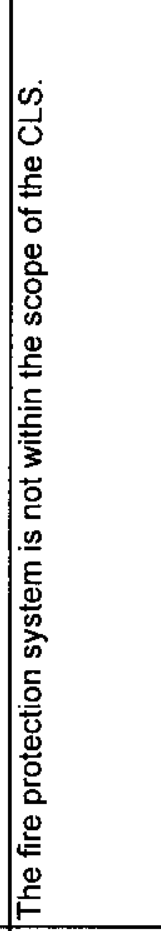 & 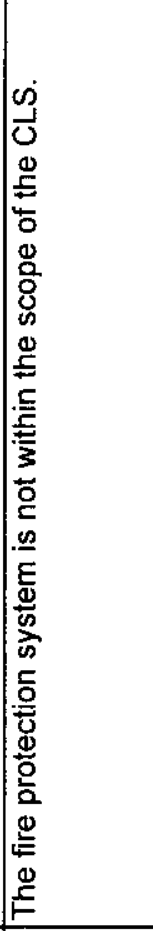 & 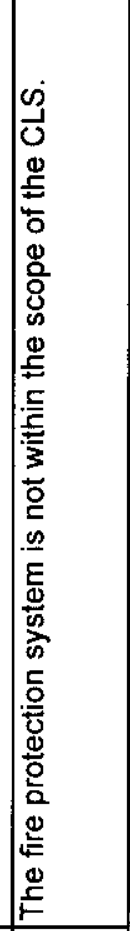 \\
\hline 苞 & $\mathbb{\mathbf { z }}$ & $\S$ & $\stackrel{\S}{\mathbf{z}}$ & & & $\mathbb{z}$ & 0 & $\frac{\$}{\mathbf{z}}$ & $\S$ & $\mathbb{\mathbf { z }}$ & $\frac{\aleph}{z}$ & 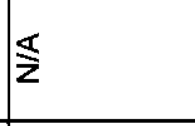 & 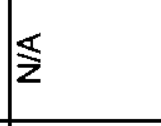 & 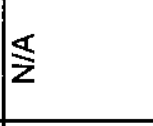 & $\mathbb{z}$ \\
\hline 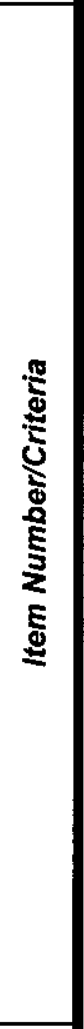 & 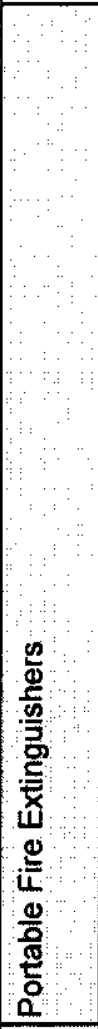 & 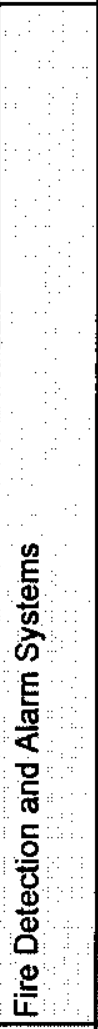 & 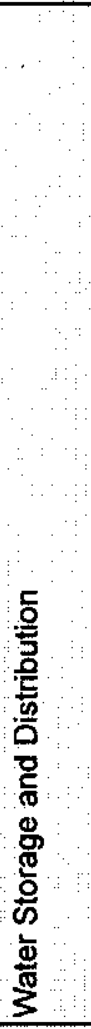 & 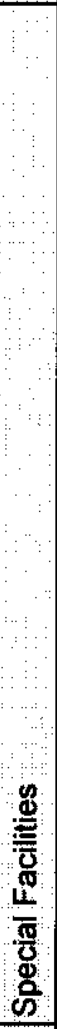 & $\begin{array}{c}\overline{\mathbf{w}} \\
\mathbf{\Phi} \\
\mathbf{g} \\
\end{array}$ & 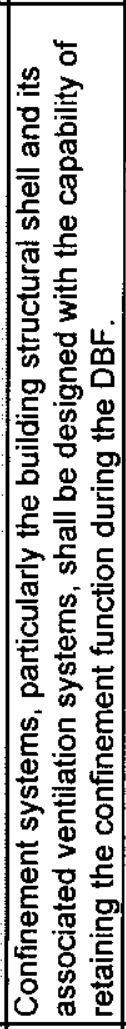 & 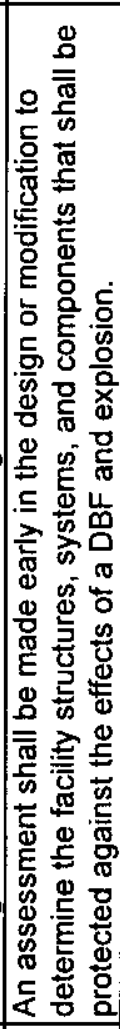 & 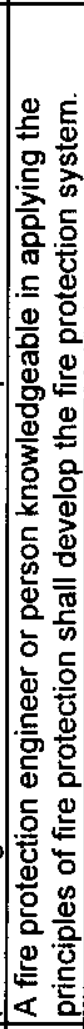 & 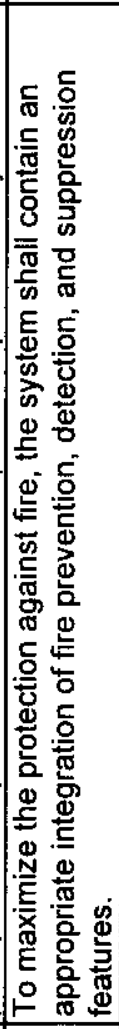 & 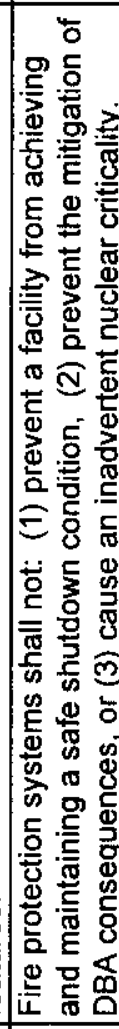 & 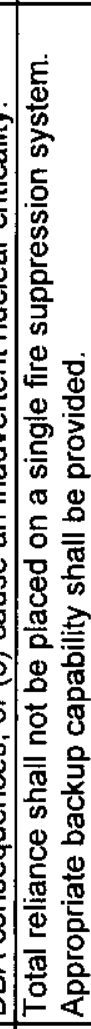 & 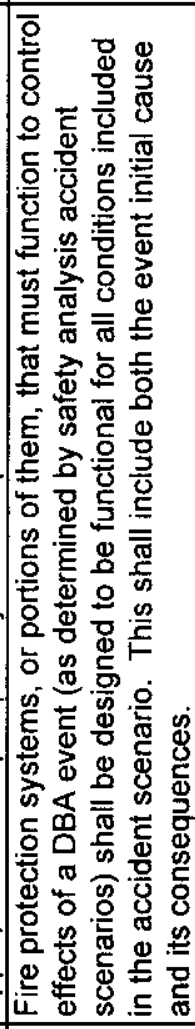 & 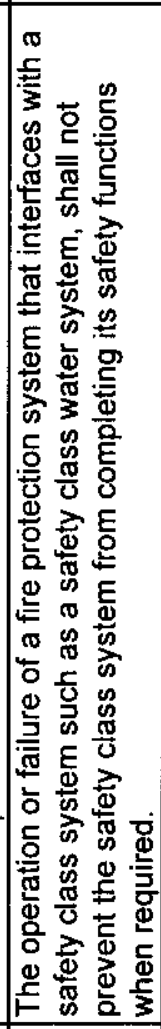 & 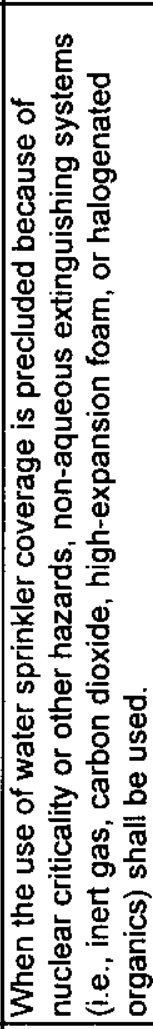 & 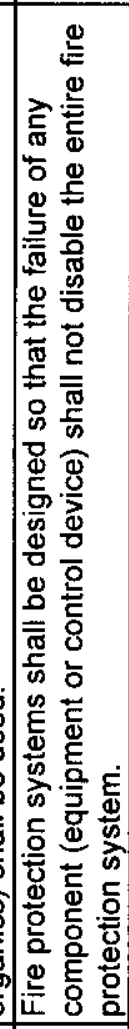 \\
\hline$\dot{0}$ & ్ํ & 요 & 志 & & & 㞻 & 怘 & $\sqrt{2}$ & 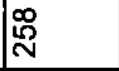 & 零 & @্ন & $\overline{\stackrel{N}{N}}$ & ల్ల & $\overparen{N}$ & 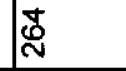 \\
\hline 丞 & है & ஓं & o̊ & \begin{tabular}{l}
8 \\
8 \\
8 \\
\hdashline \\
5
\end{tabular} & $\begin{array}{l}0 \\
8 \\
8 \\
8 \\
6 \\
6\end{array}$ & 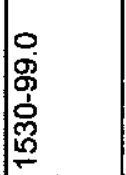 & $g$ & 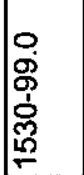 & (8) & 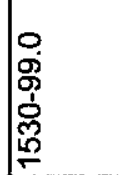 & 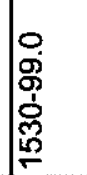 & 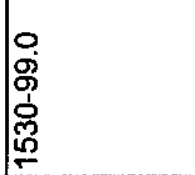 & क् & 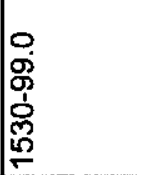 & 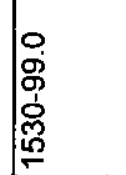 \\
\hline
\end{tabular}


SNF-4775, Rev. 2

Project A.5/A.6

APPENDIX A

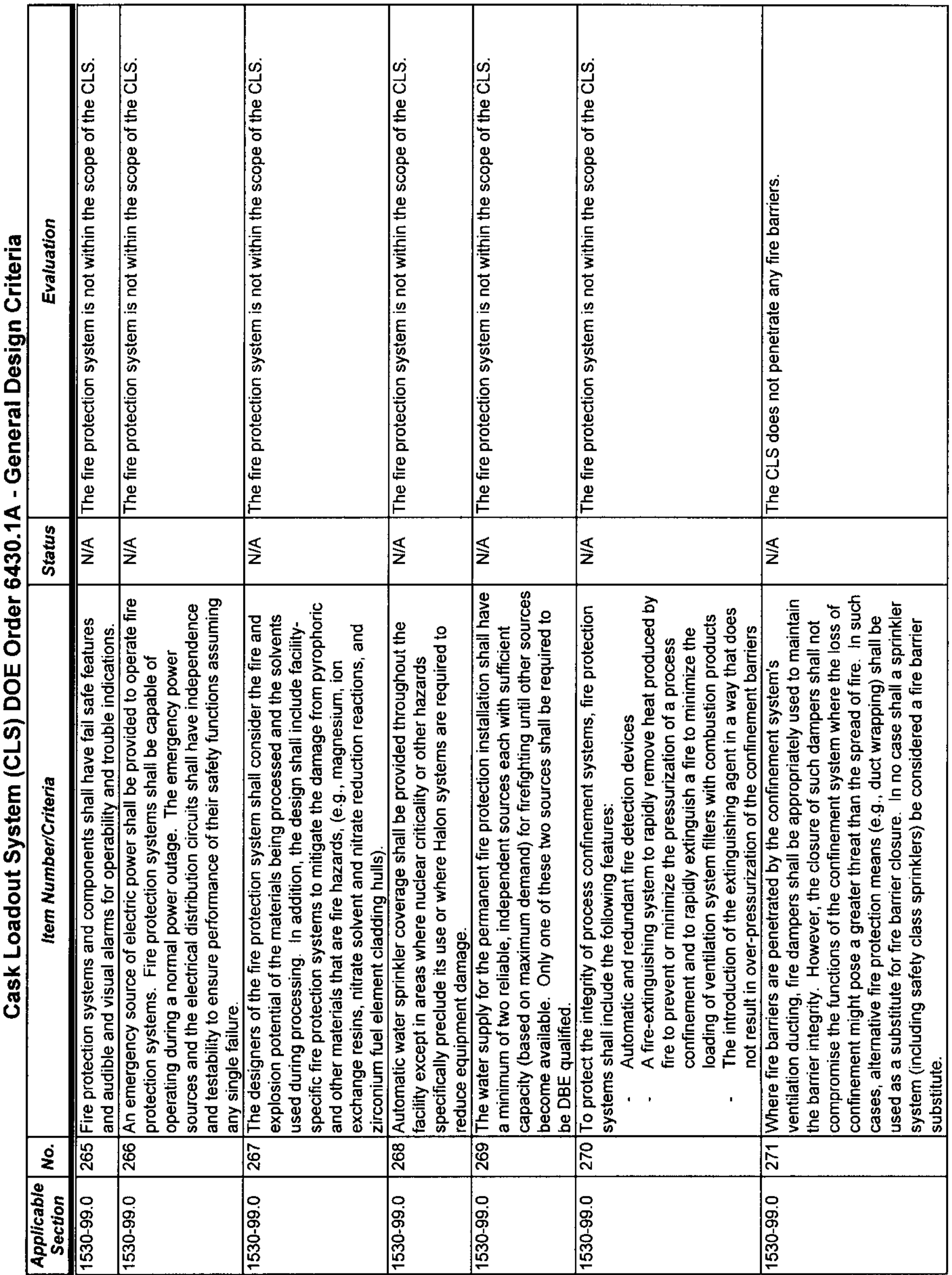


SNF-4775, Rev. 2

Project A.5/A.6

APPENDIX A

\begin{tabular}{|c|c|c|c|c|c|c|c|c|c|c|c|c|c|c|c|c|}
\hline 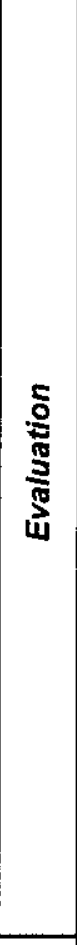 & 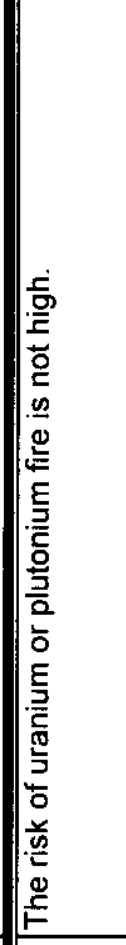 & 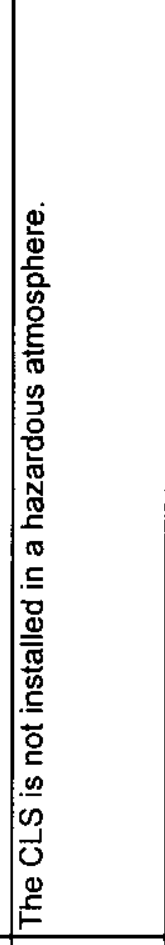 & 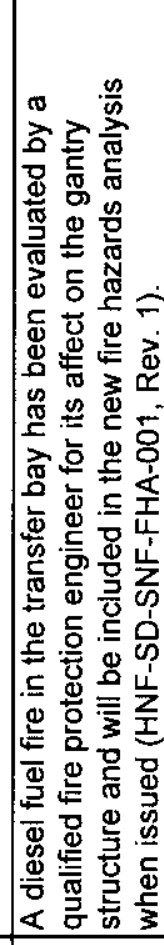 & 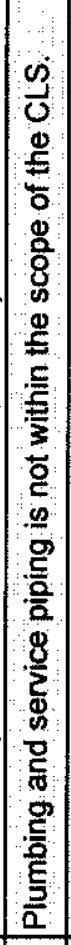 & 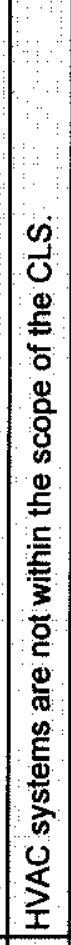 & 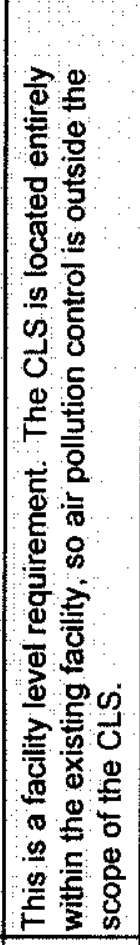 & 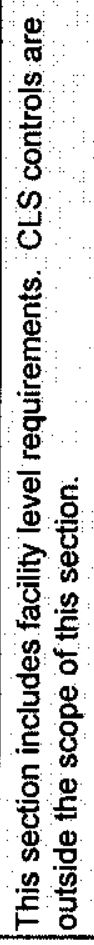 & & & 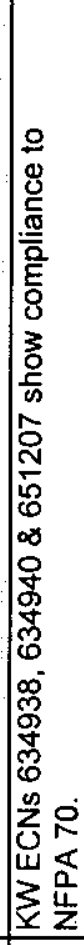 & 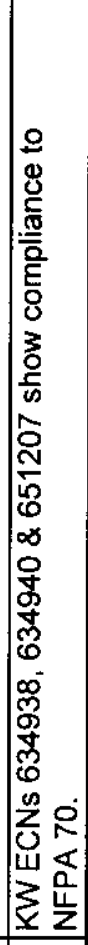 & 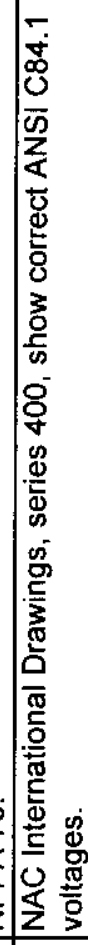 & 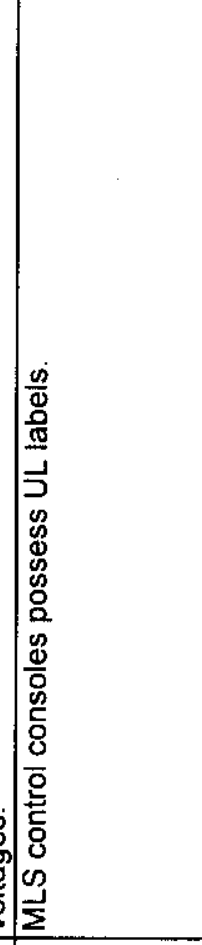 & 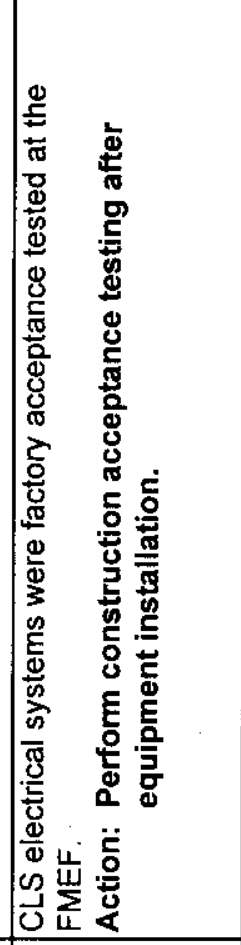 & & \\
\hline 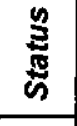 & $\mid \S$ & $\frac{\$}{z}$ & 0 & $\$$ & $\$$ & 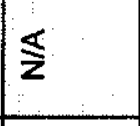 & 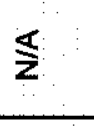 & & & 0 & 0 & 0 & 0 & $\frac{z}{u}$ & & \\
\hline 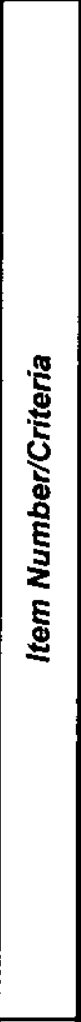 & 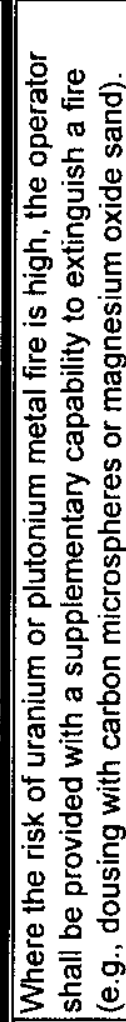 & 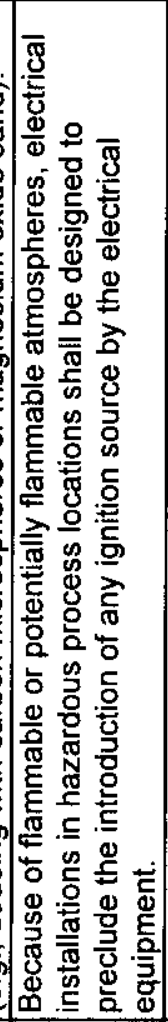 & 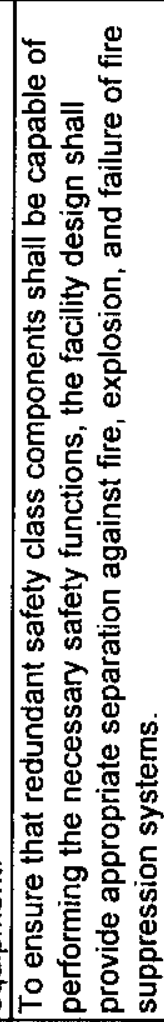 & $\begin{array}{l}\frac{D}{2} \\
\frac{2}{2} \\
\frac{8}{2} \\
\frac{D}{5} \\
\frac{5}{2} \\
\frac{5}{5}\end{array}$ & 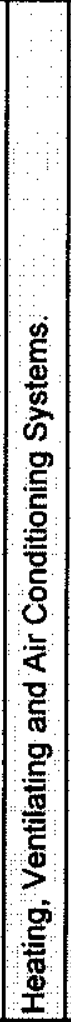 & 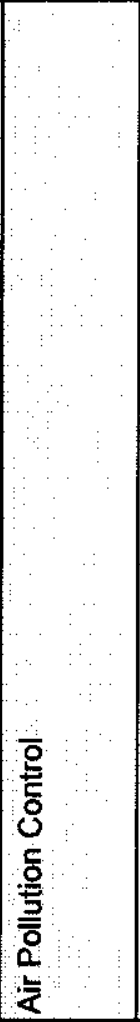 & $\begin{array}{l}\text { 0. } \\
\text { 늘 } \\
0 \\
0\end{array}$ & 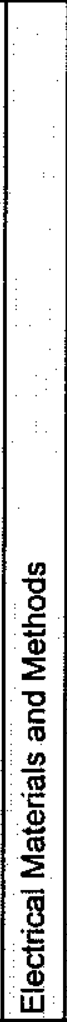 & 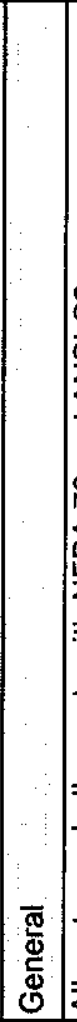 & 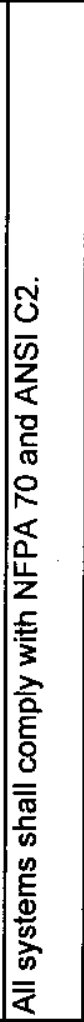 & 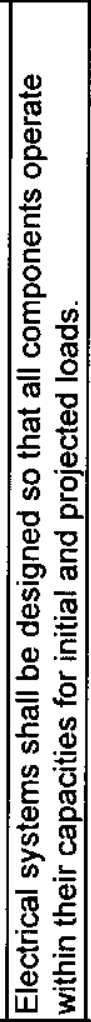 & 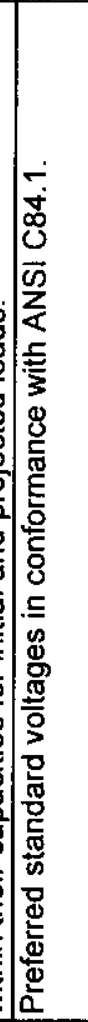 & 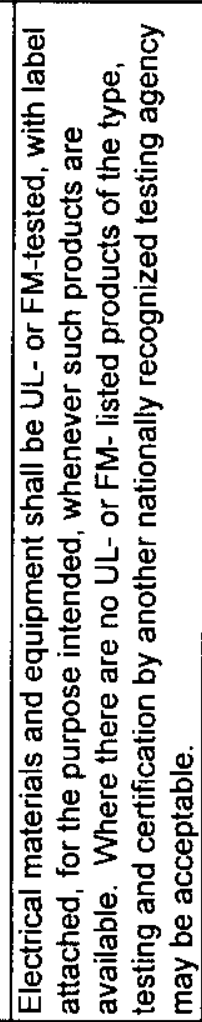 & 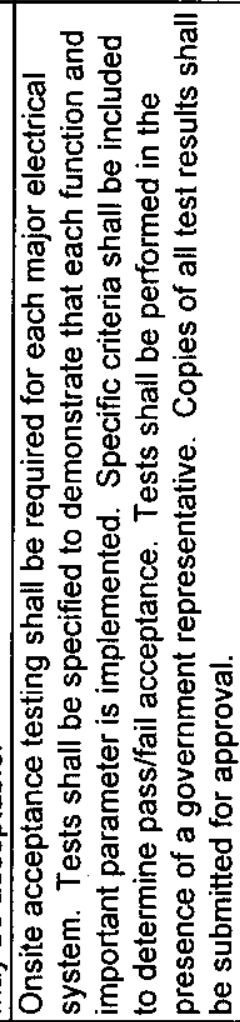 & 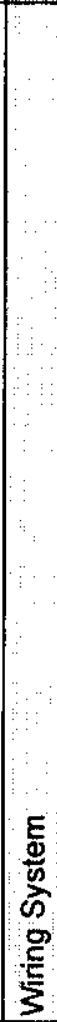 & 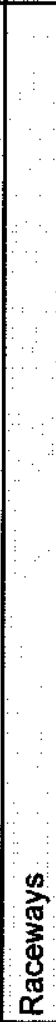 \\
\hline i & $\stackrel{N}{N}$ & $\stackrel{p}{N}$ & $\stackrel{N}{N}$ & ก & $\stackrel{0}{2}$ & $\hat{N}$ & $\stackrel{\infty}{\sim}$ & & & $\begin{array}{l}\text { D } \\
\text { N }\end{array}$ & : & $\overline{\mathbf{N}}$ & ్ָ & 怘 & & \\
\hline : & o & 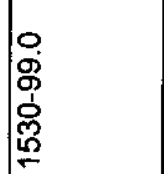 & 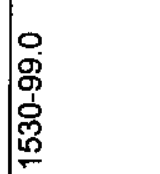 & 은 & ํㅜㅇ & 急 & 禺 & 8 & 융 & 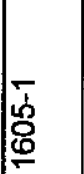 & | & 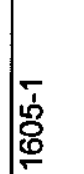 & 象 & 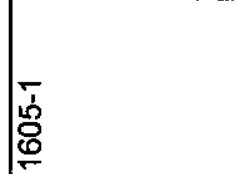 & 용 & \%̊ \\
\hline
\end{tabular}


SNF-4775, Rev. 2

Project A.5/A.6

APPENDIX A

\begin{tabular}{|c|c|c|c|c|c|c|c|c|c|c|c|c|c|c|c|c|c|c|c|}
\hline 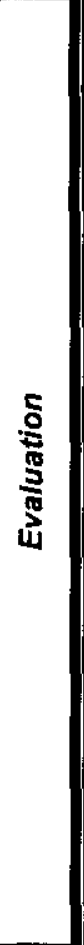 & & 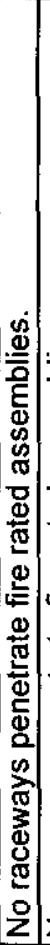 & 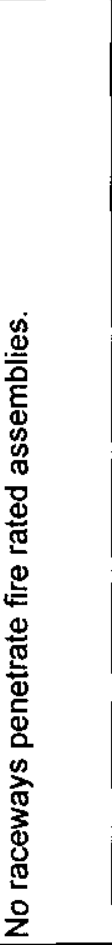 & 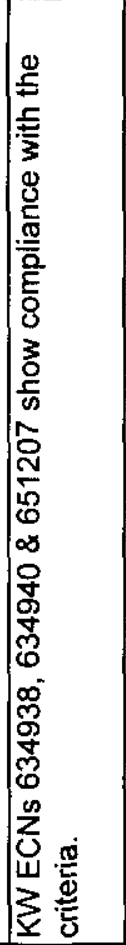 & & 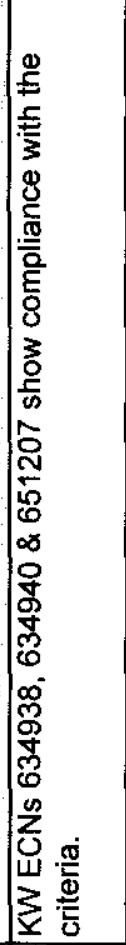 & 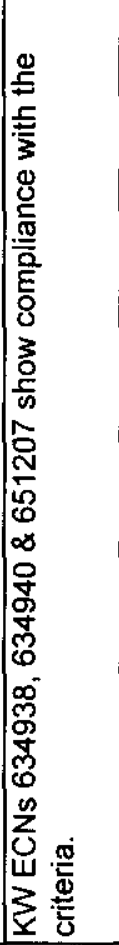 & 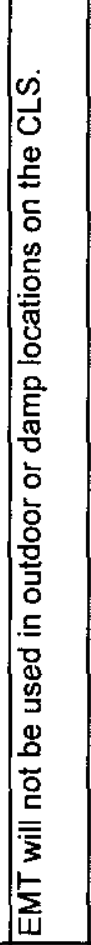 & & 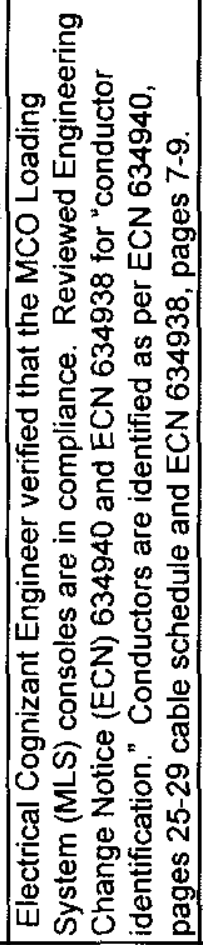 & & 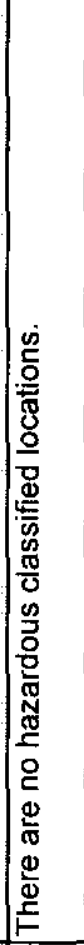 & & 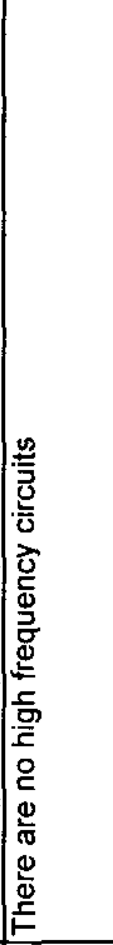 & 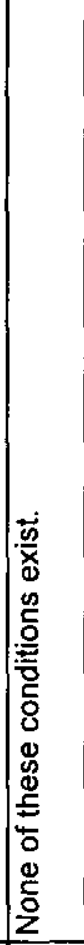 & & 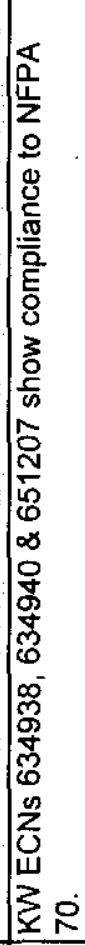 & & 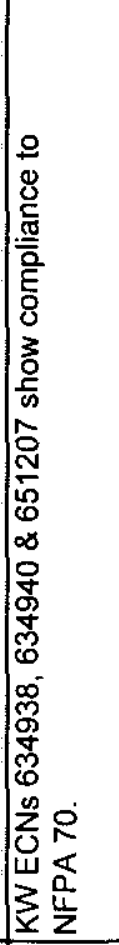 \\
\hline 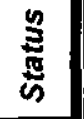 & & $\$$ & 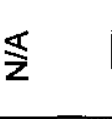 & 0 & & 10 & 0 & $\mathbb{\mathbf { z }}$ & & 0 & & $\frac{\$}{z}$ & & $\$ \S$ & $\$$ & & 10 & & 0 \\
\hline 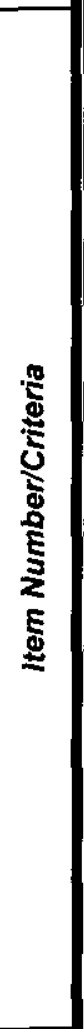 & ब्ञ & 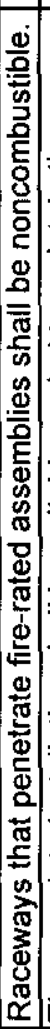 & 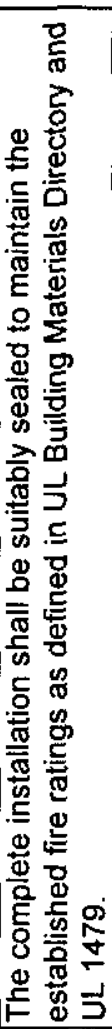 & 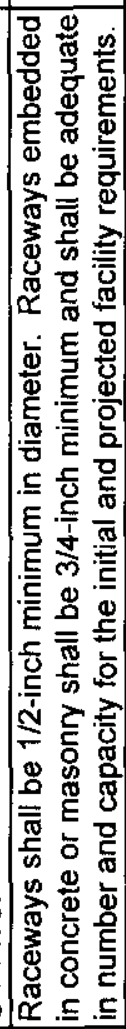 & 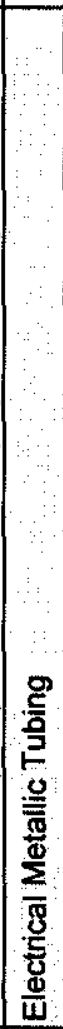 & 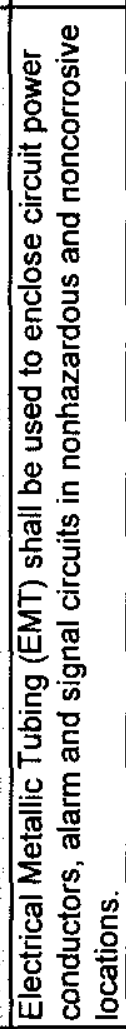 & 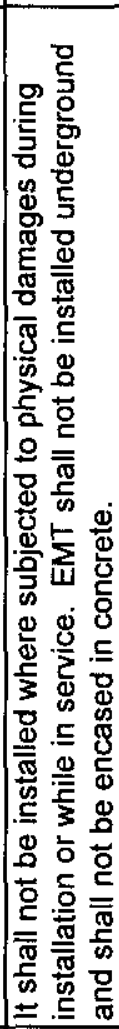 & 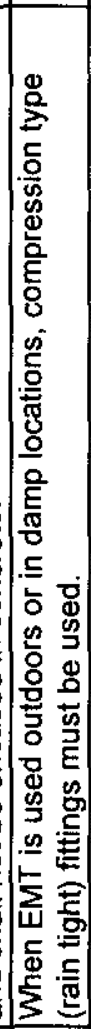 & 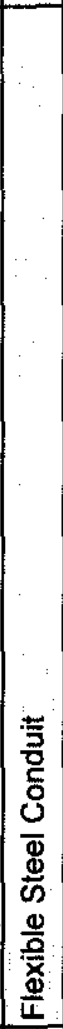 & 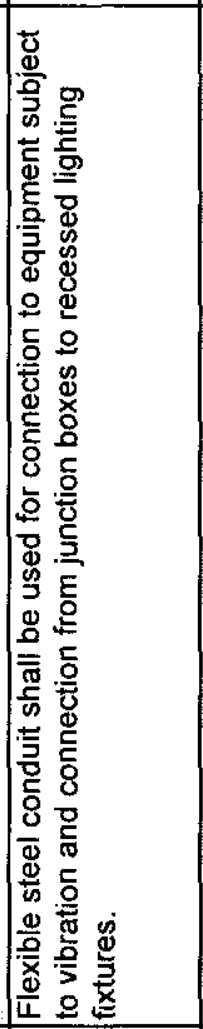 & 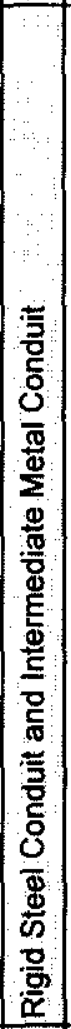 & 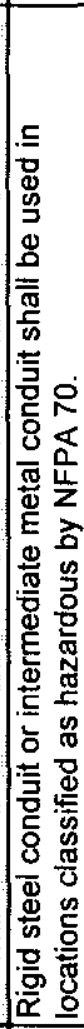 & हE & 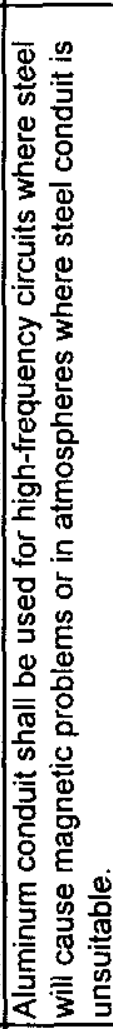 & 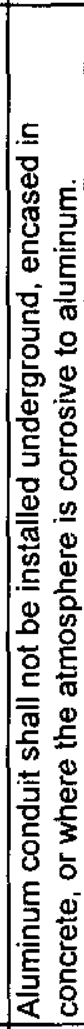 & 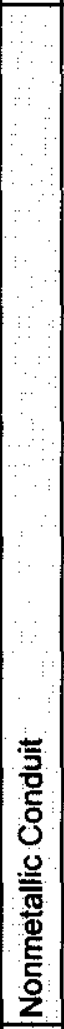 & 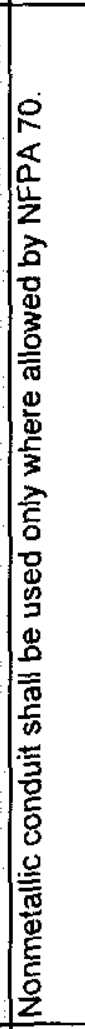 & 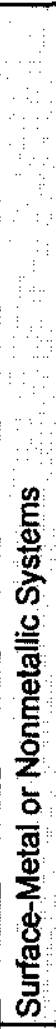 & 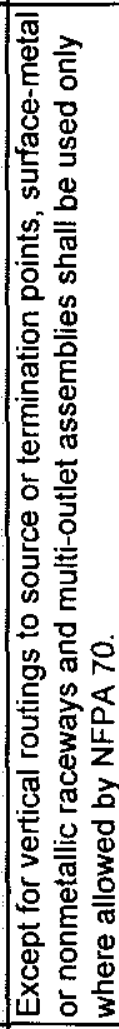 \\
\hline$\stackrel{0}{2}$ & & $N$ & $\mathscr{W}_{\substack{\infty \\
N}}$ & 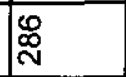 & & 隹 & $\begin{array}{l}\infty \\
\stackrel{\infty}{N} \\
\end{array}$ & : & & 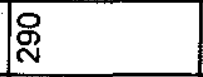 & & 汤 & & $\widetilde{\widetilde{N}}$ & న్న & & 蛋 & & $\stackrel{2}{2}$ \\
\hline 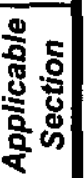 & 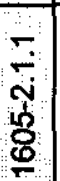 & 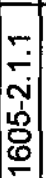 & & 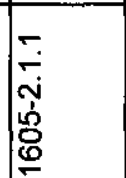 & 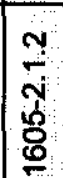 & 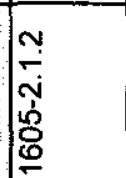 & 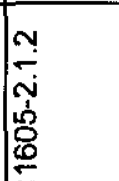 & 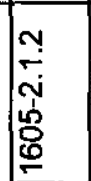 & 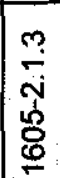 & 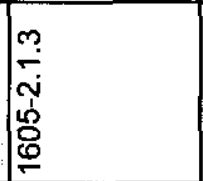 & 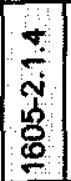 & 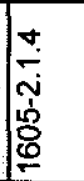 & \% & 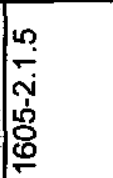 & & 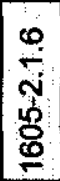 & 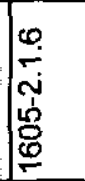 & ते & 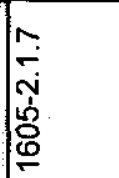 \\
\hline
\end{tabular}


SNF-4775, Rev. 2

Project A.5/A.6

APPENDIX A

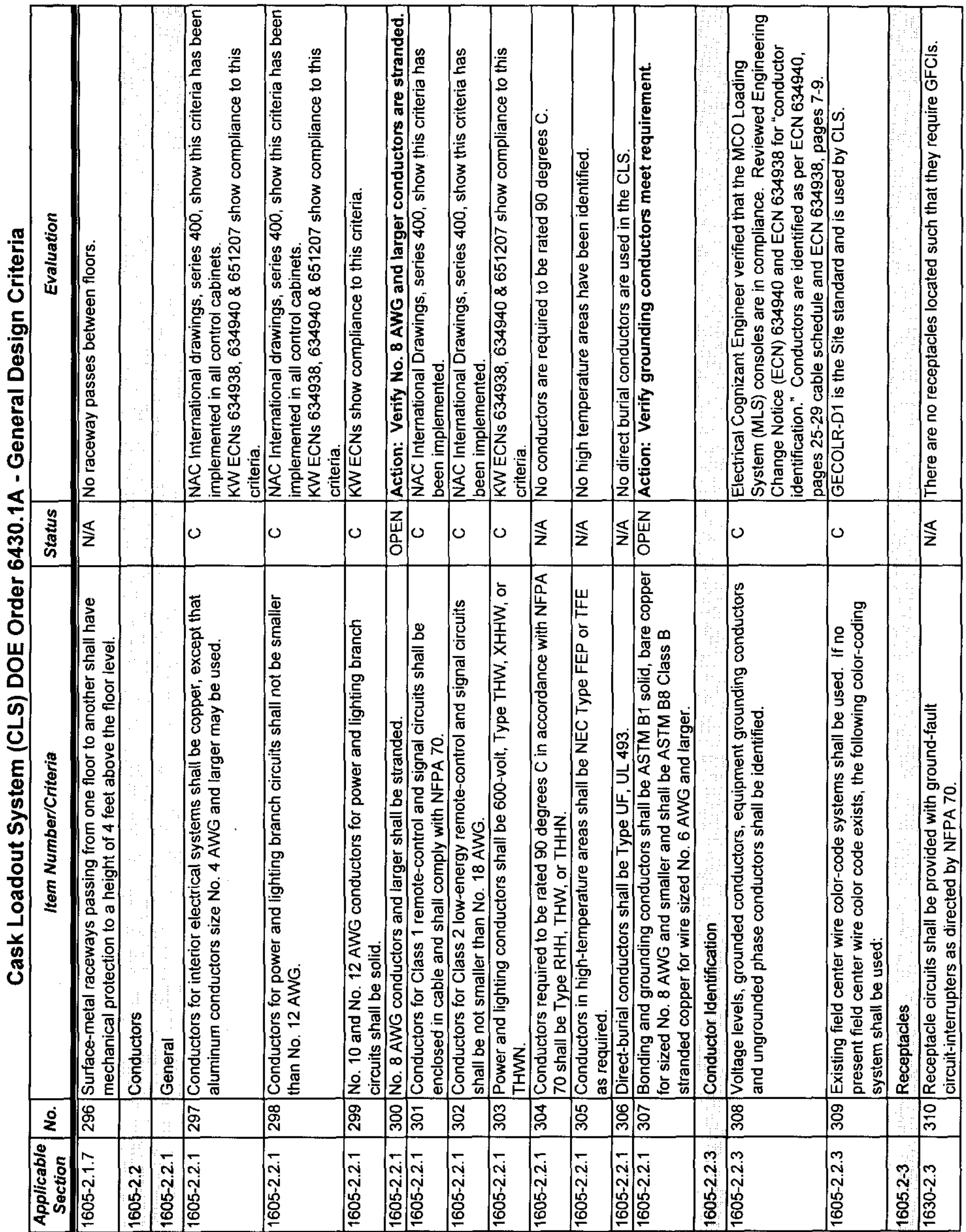


SNF-4775, Rev. 2

Project A.5/A.6

APPENDIX A

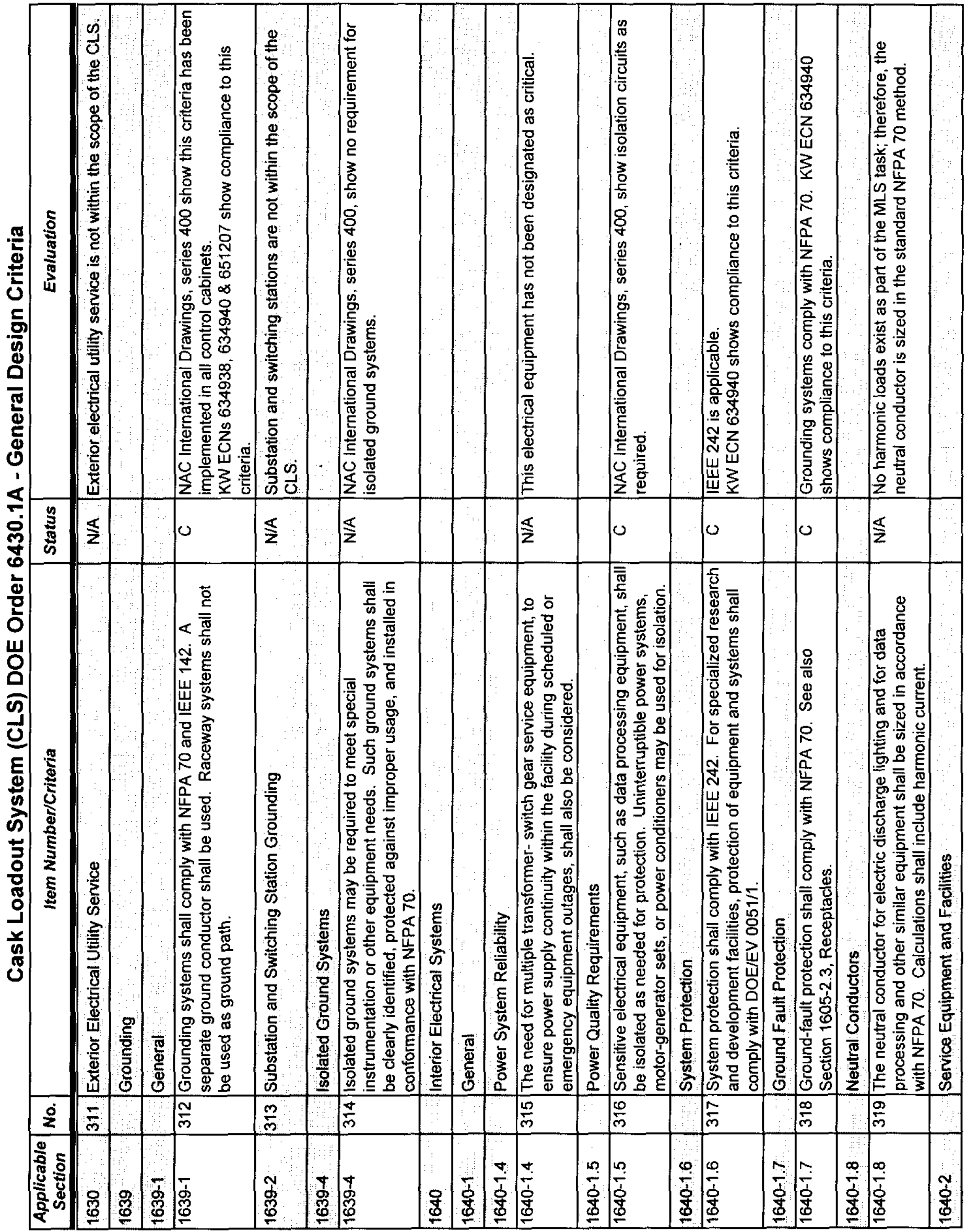


SNF-4775, Rev. 2

Project A.5/A.6

APPENDIX $A$

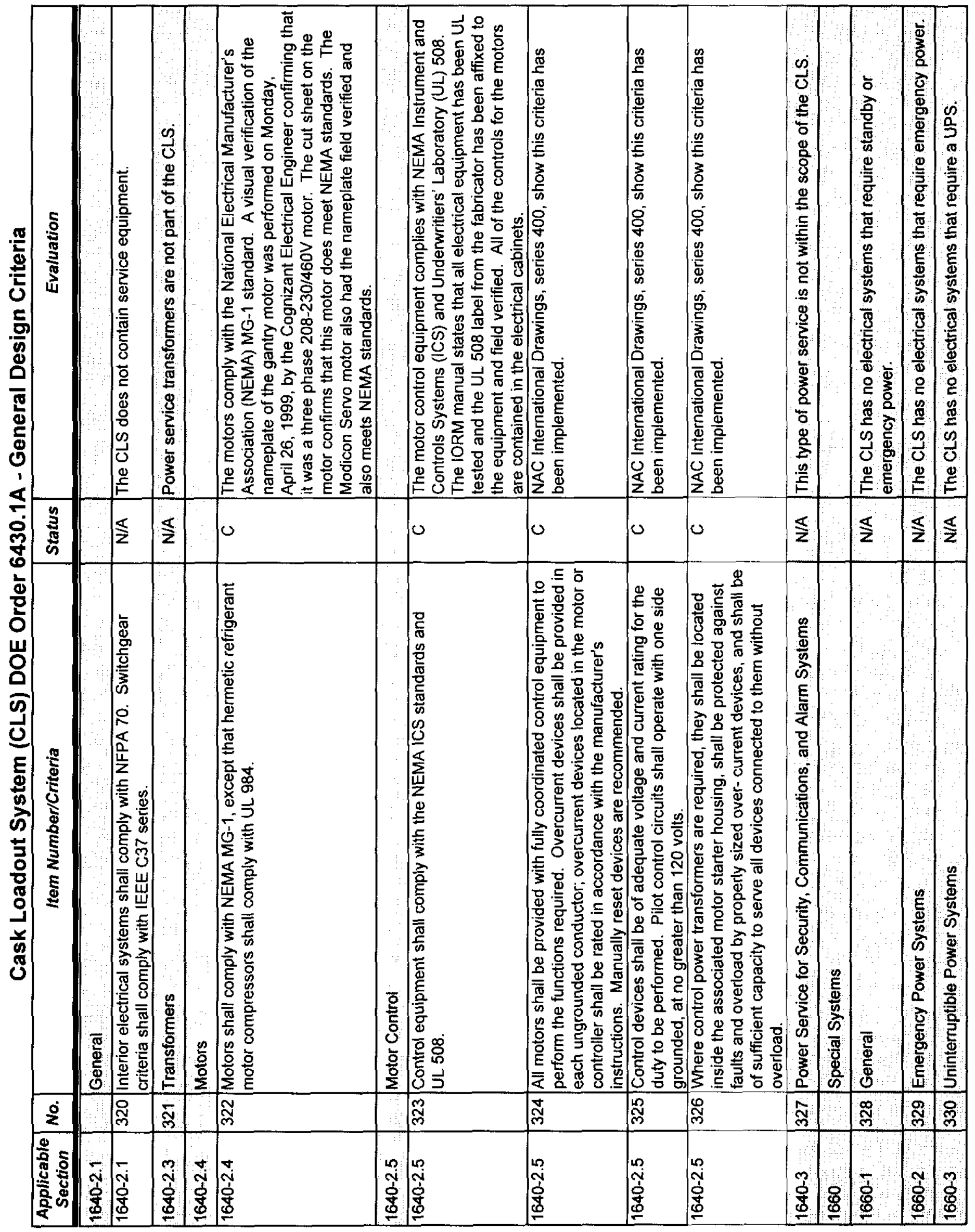


SNF-4775, Rev. 2

Project A.5/A.6

APPENDIX $A$

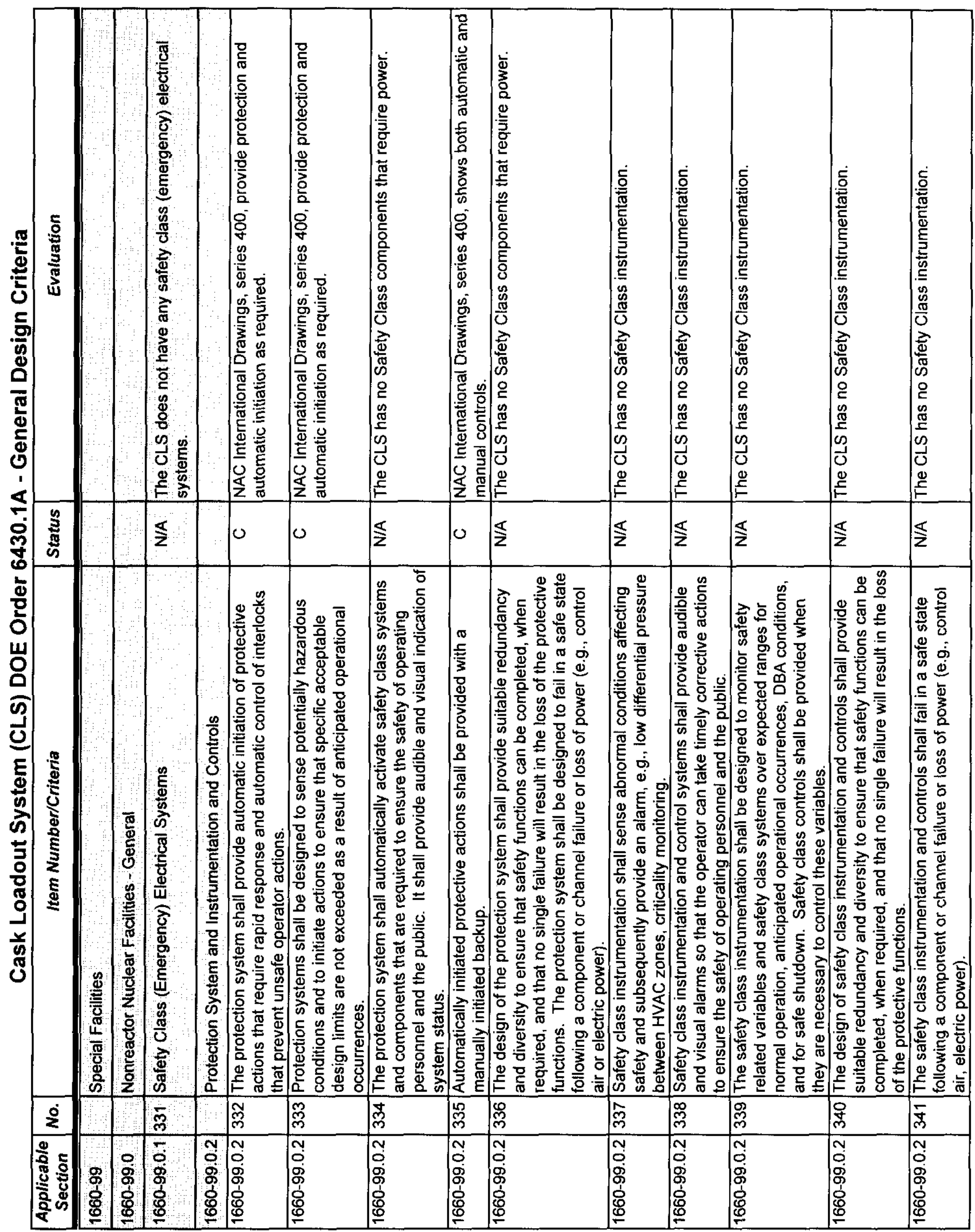


SNF-4775, Rev. 2

Project A.5/A.6

APPENDIX A

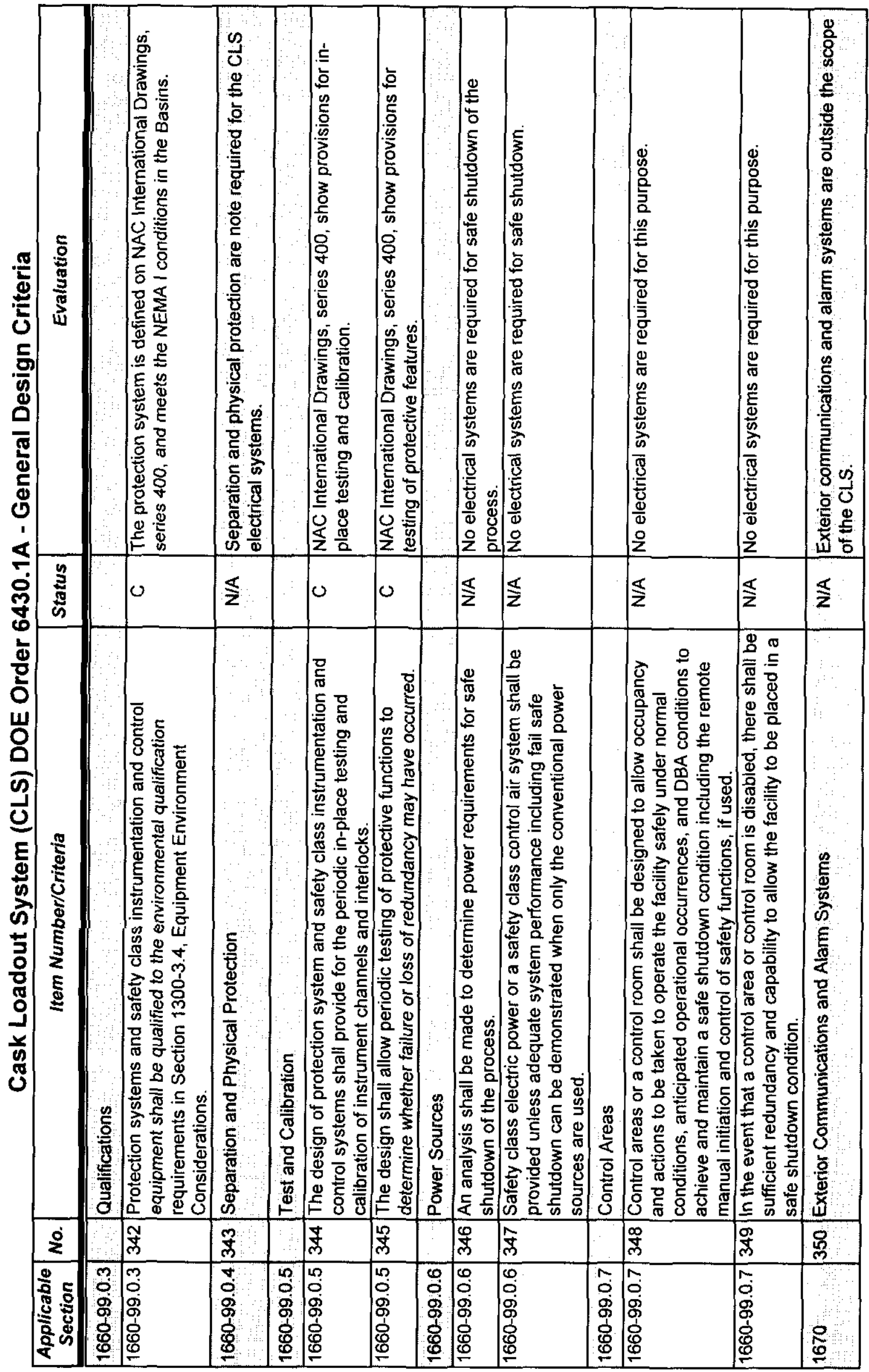


SNF-4775, Rev. 2

Project A.5/A.6

APPENDIX B

CRANES /OTHER MODIFICATIONS

DOE ORDER 6430.1A -- GENERAL DESIGN CRITERIA

CONSISTING OF 99 PAGES

INCLUDING COVERSHEET

Page B-1 


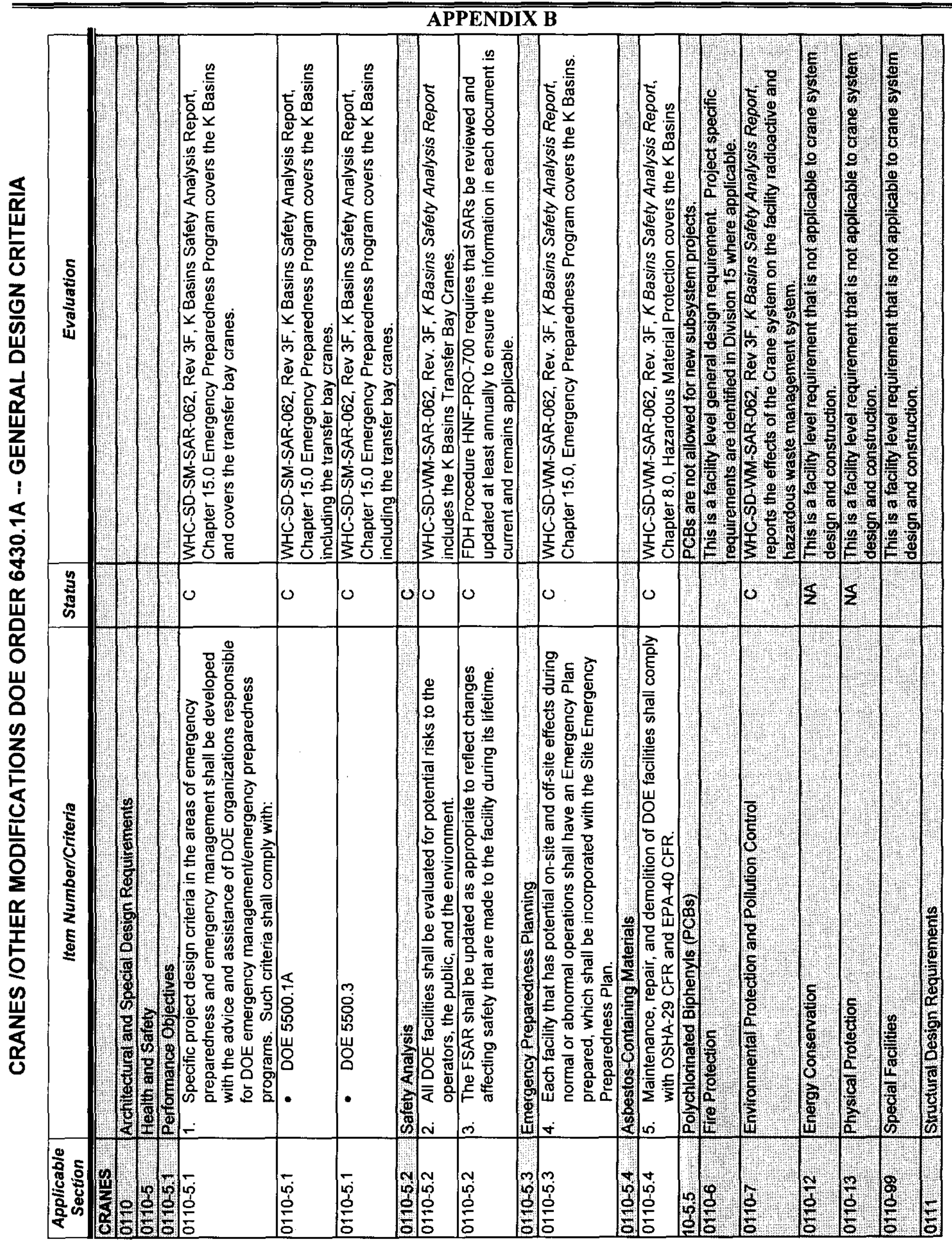




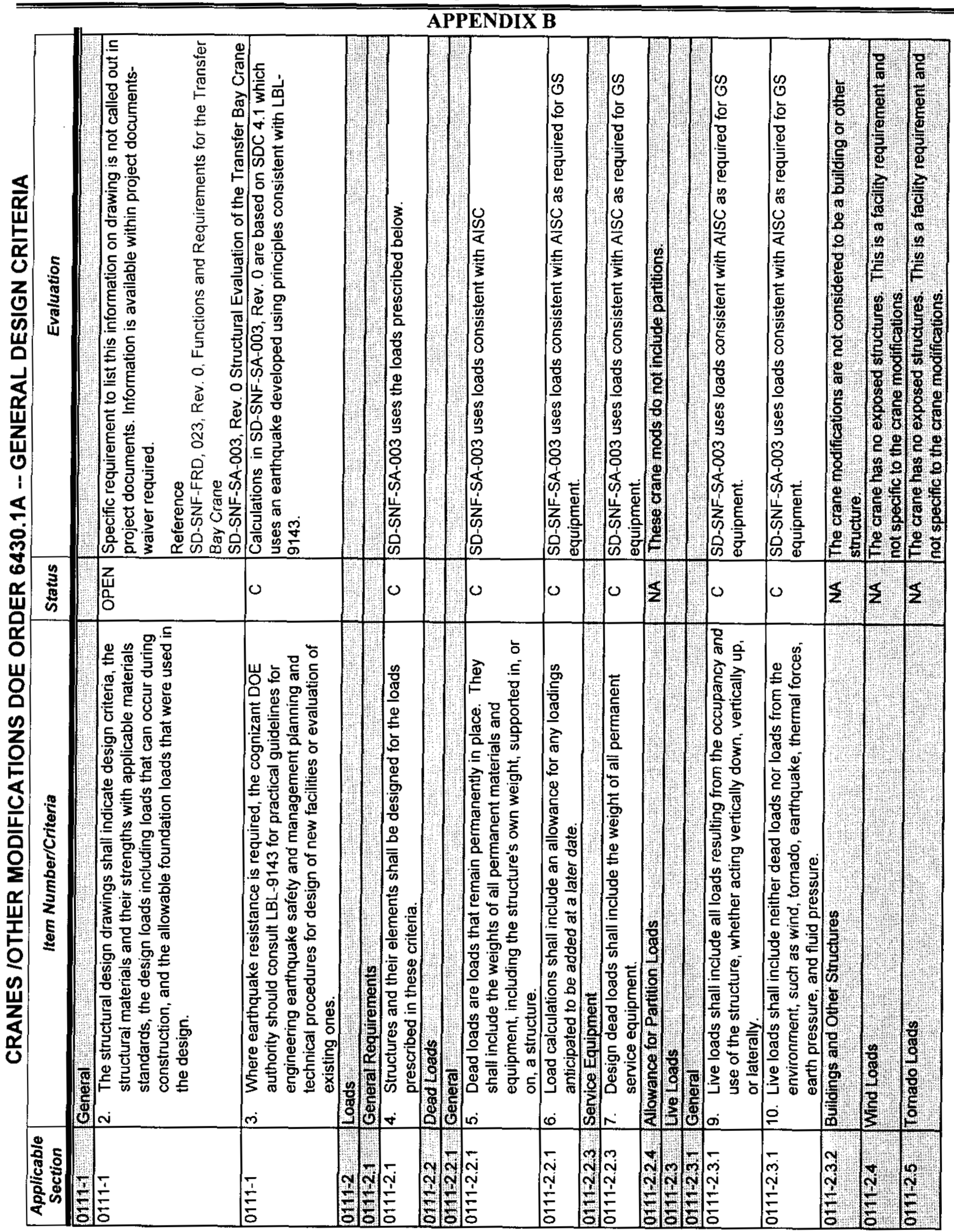

Page B-3 
SNF-4775, Rev. 2

Project A.5/A.6

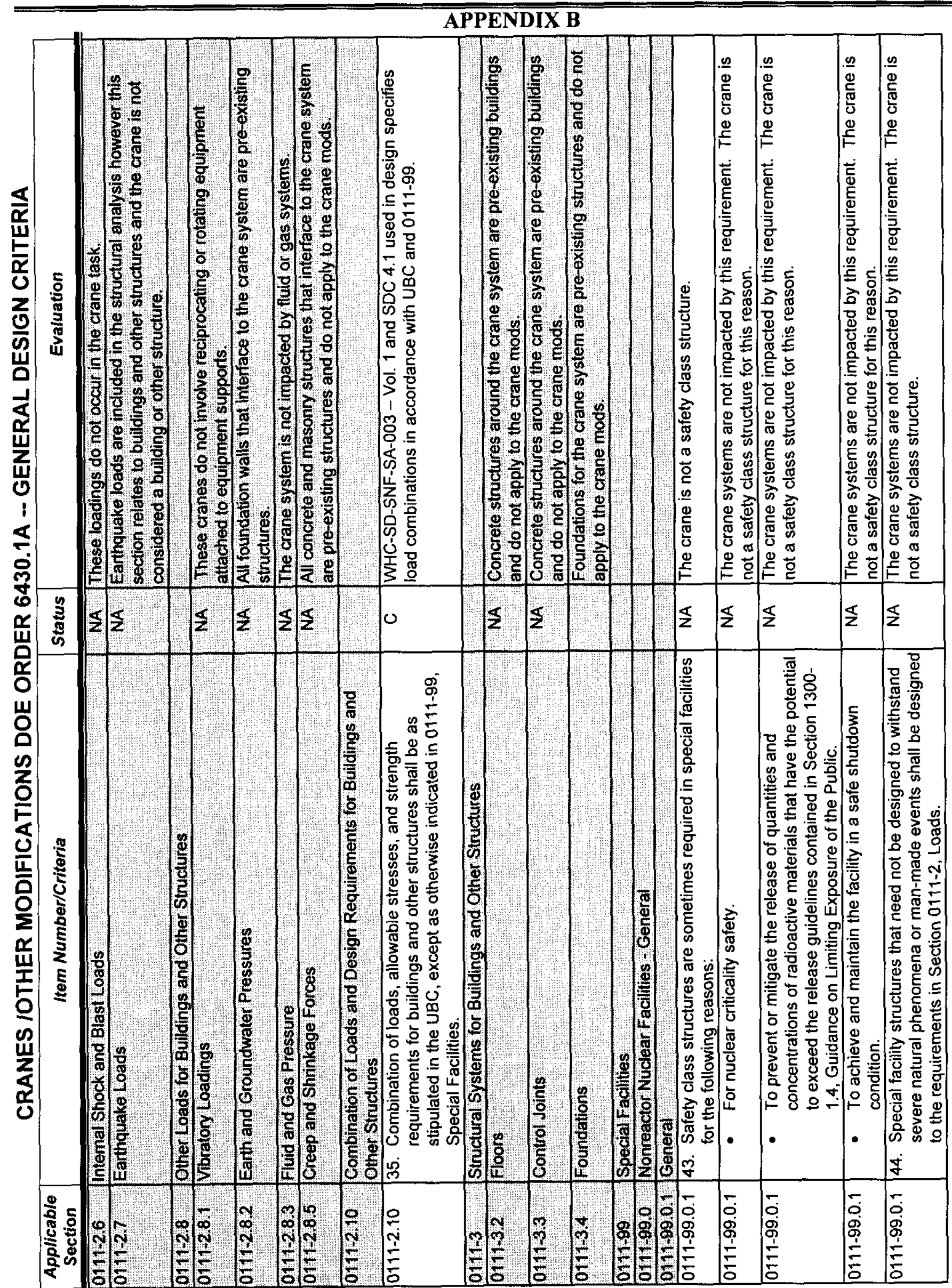

Page B-4 


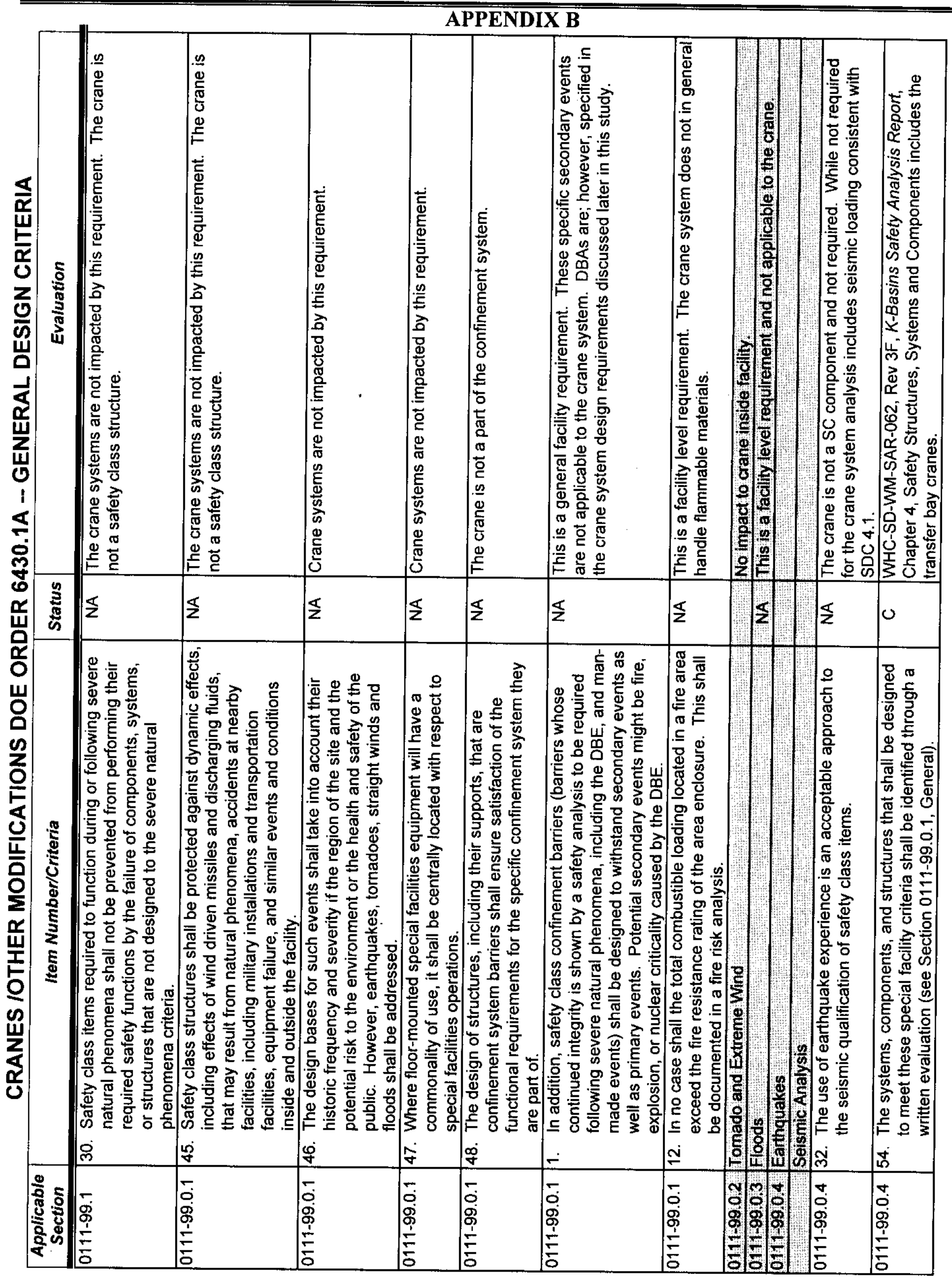


SNF-4775, Rev. 2

Project A.5/A.6

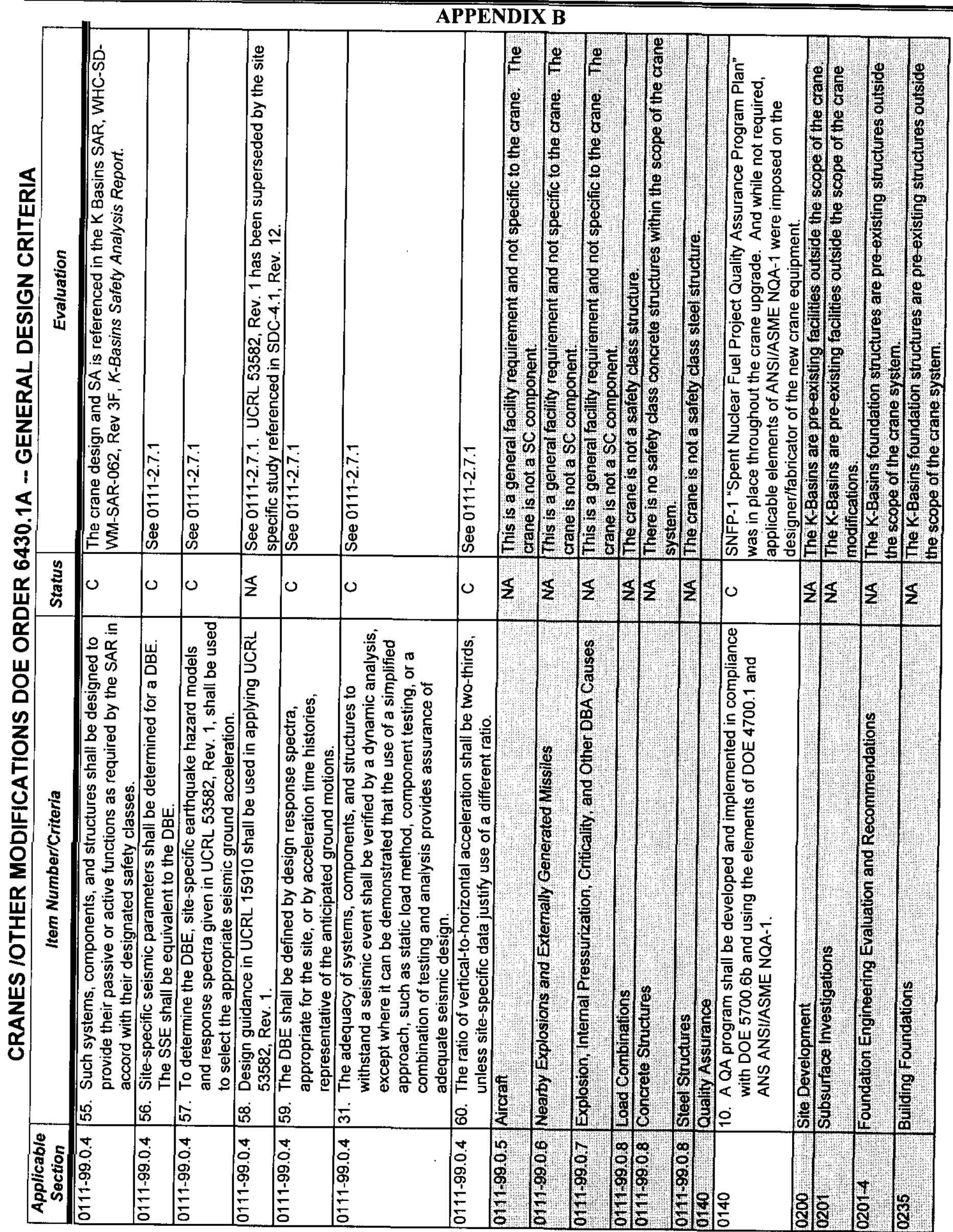

Page B-6 


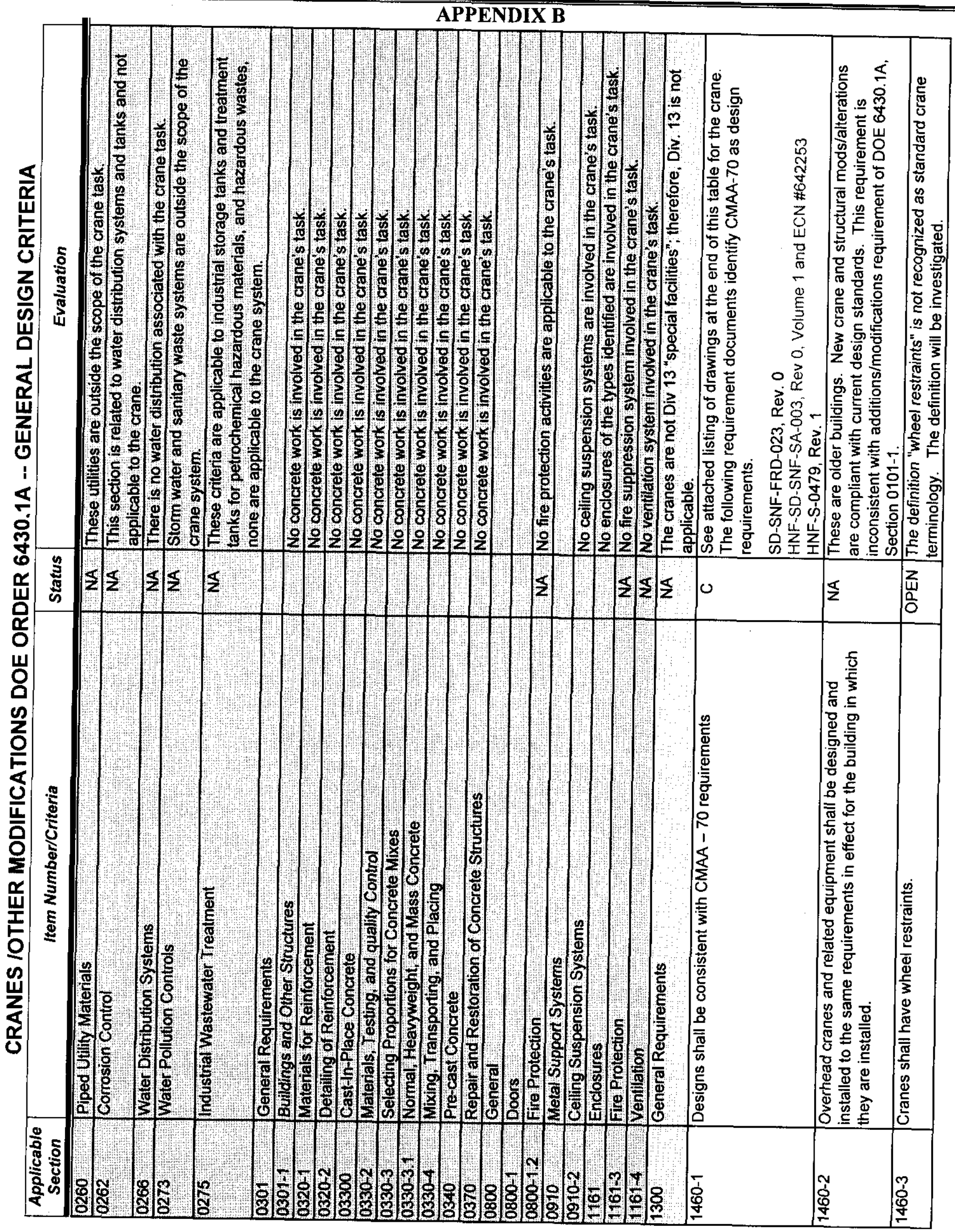


SNF-4775, Rev. 2

Project A.5/A.6

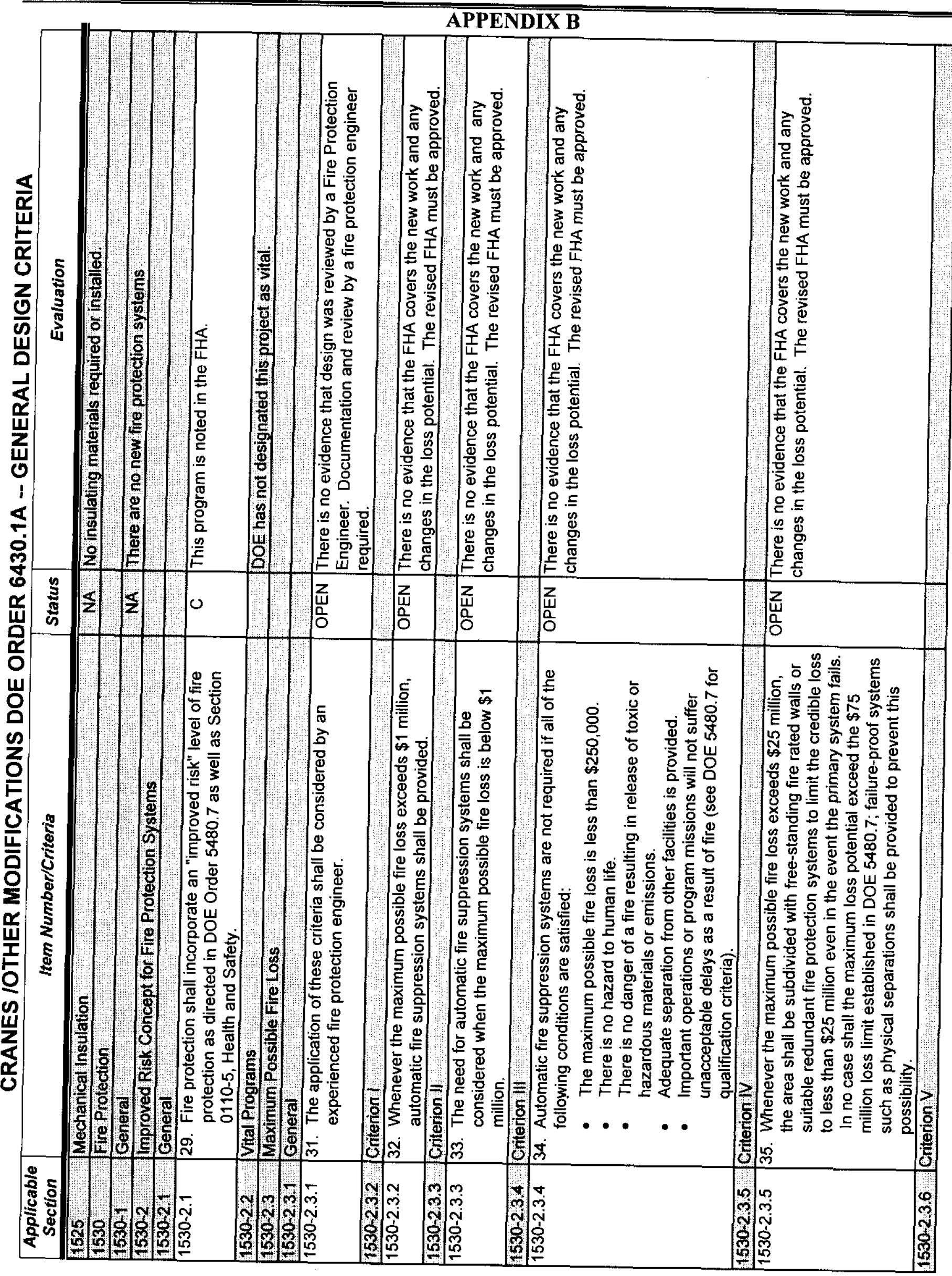

Page B-8 
SNF-4775, Rev. 2

Project A.5/A.6

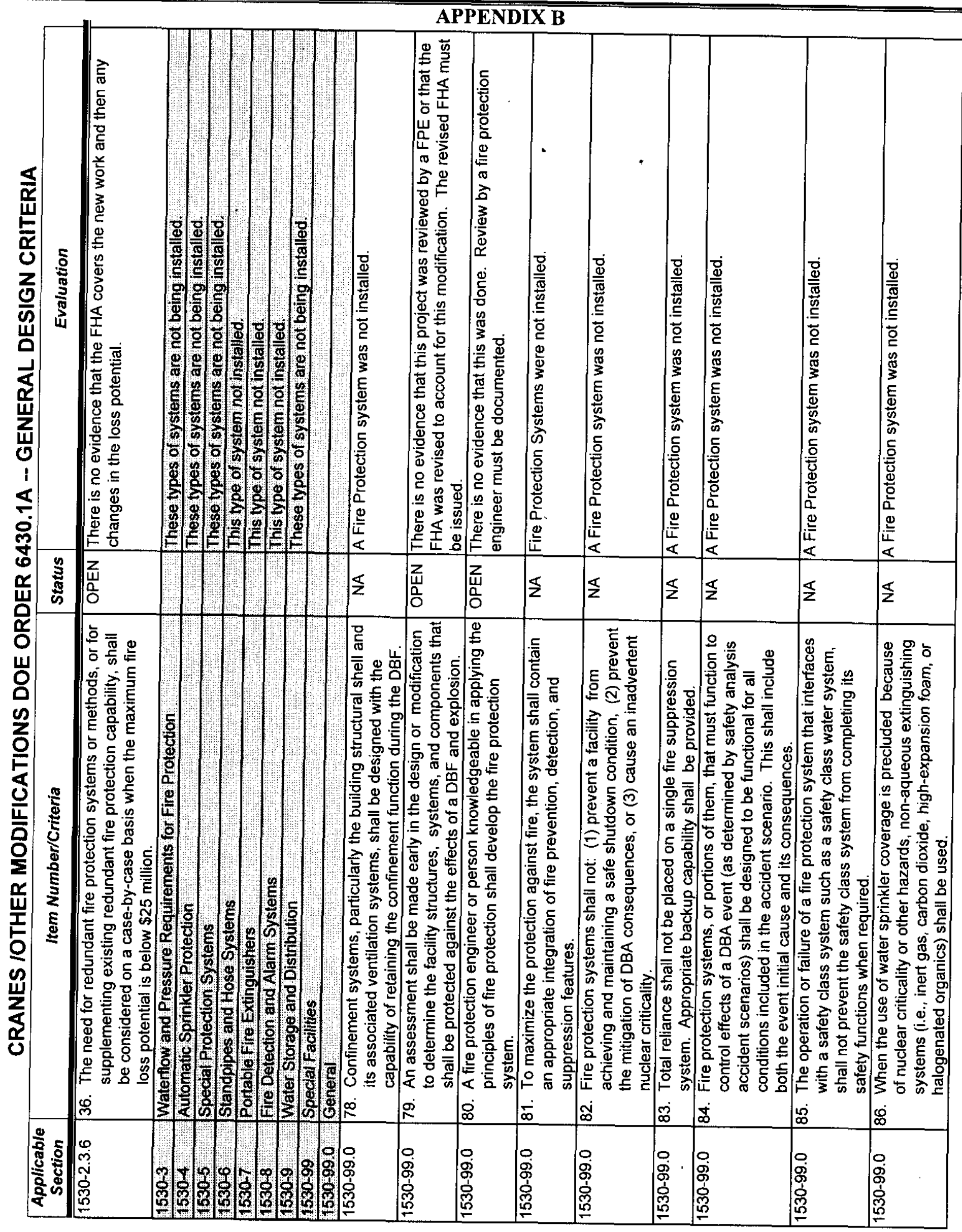

Page B-9 


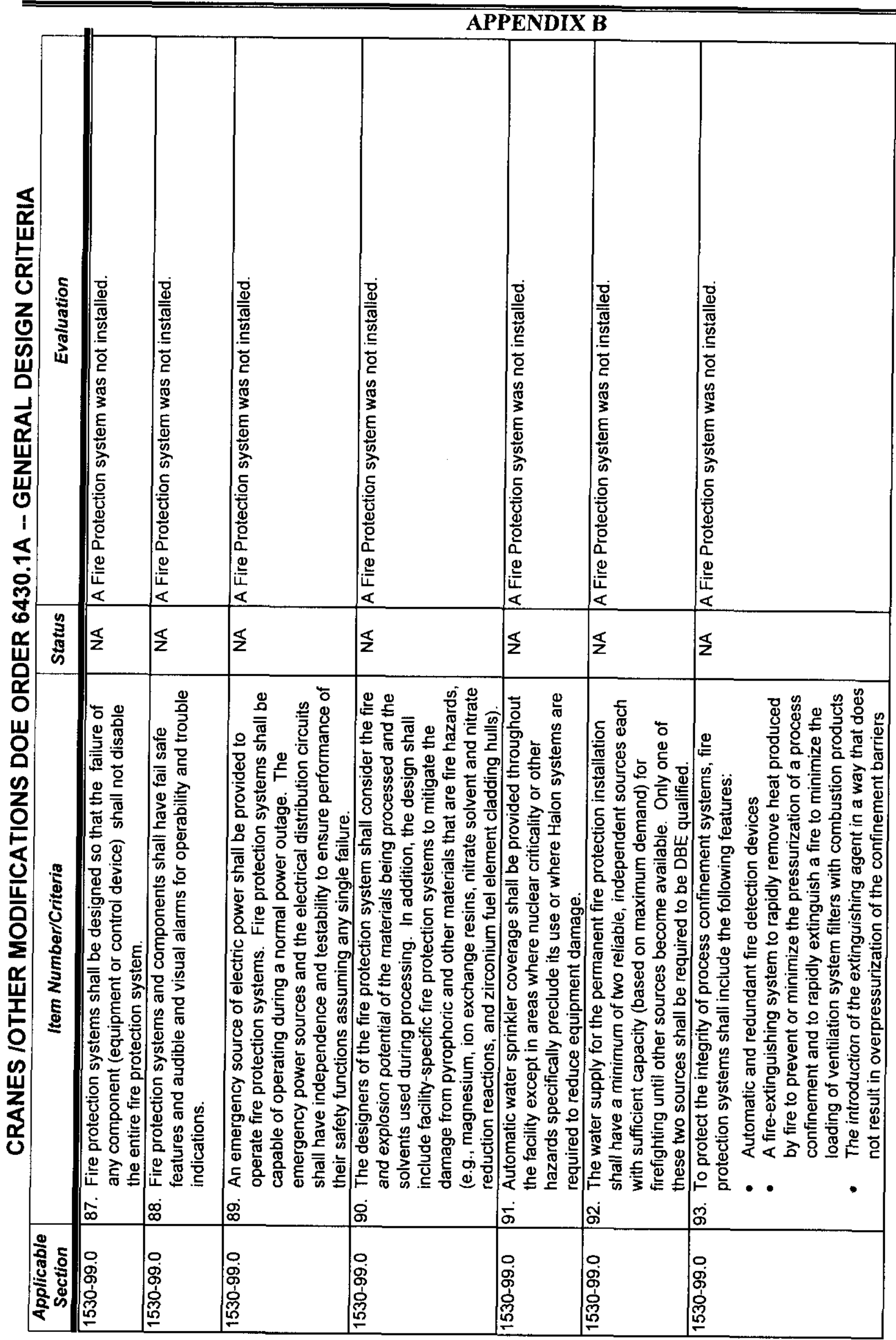


SNF-4775, Rev. 2

Project A.5/A.6

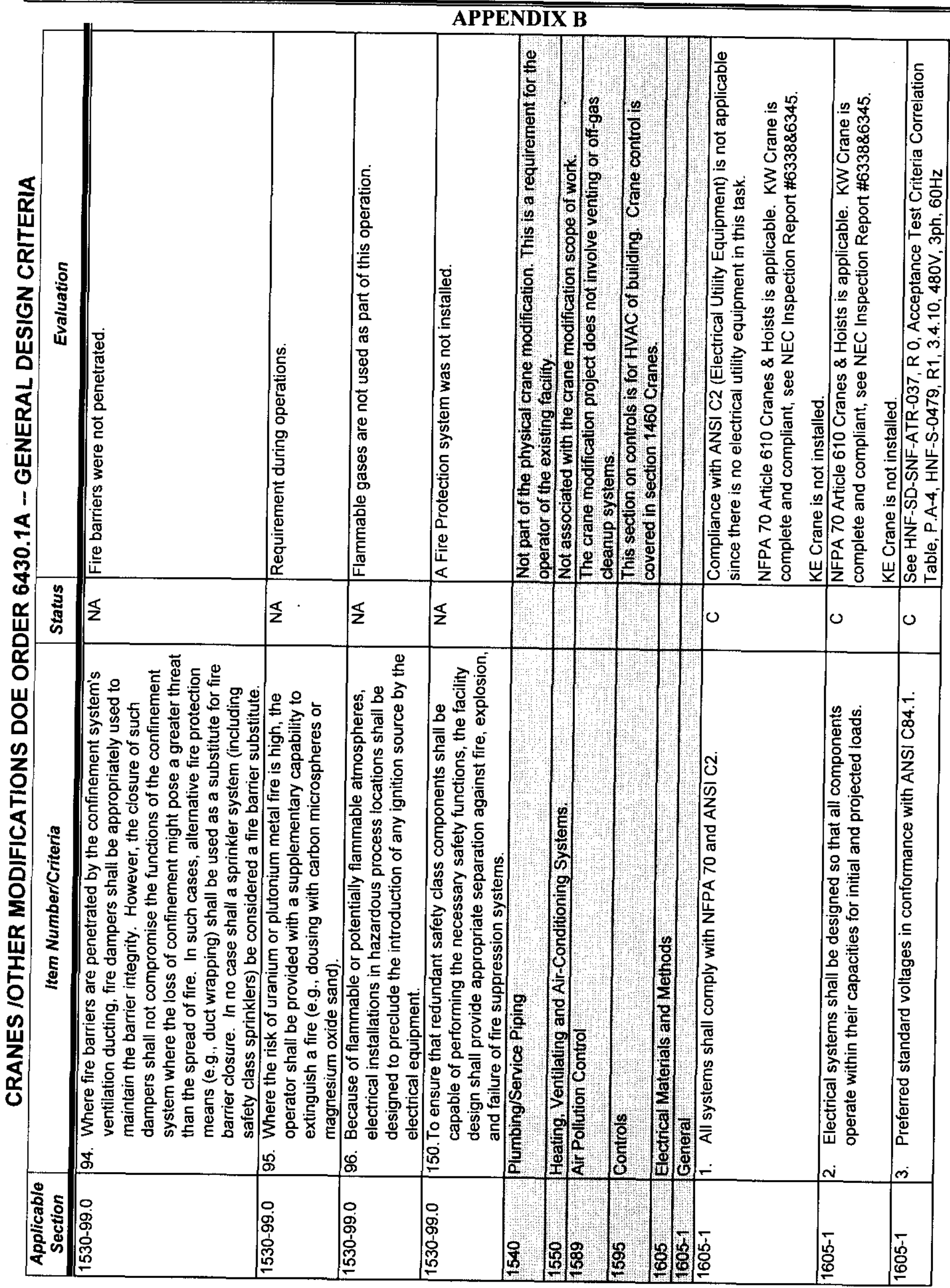

Page B-11 
SNF-4775, Rev. 2

Project A.5/A.6

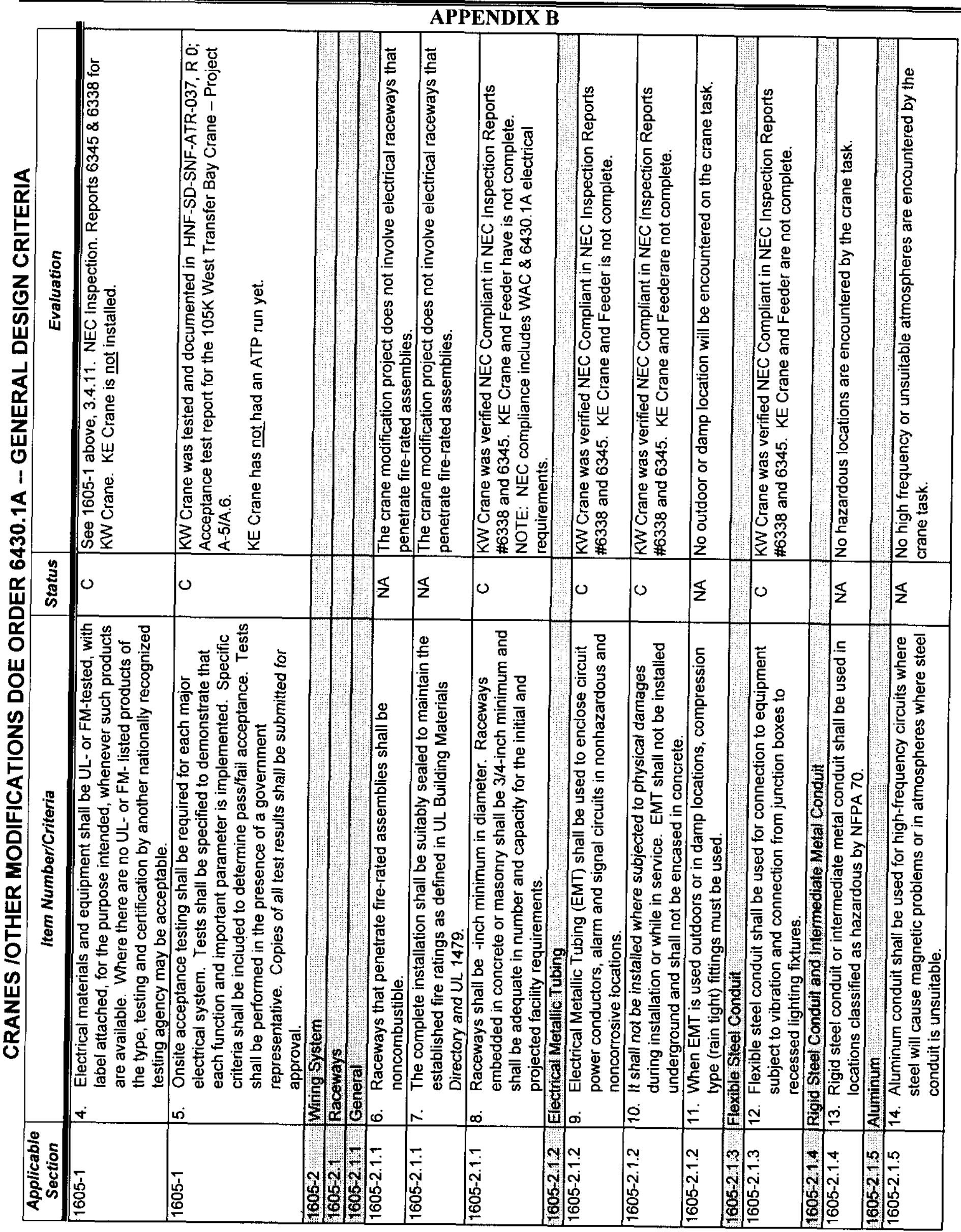

Page B-12 
SNF-4775, Rev. 2

Project A.5/A.6

\begin{tabular}{|c|c|c|c|c|c|c|c|c|c|c|c|c|c|c|c|c|c|c|c|c|}
\hline \multicolumn{21}{|c|}{ APPENDIX B } \\
\hline 売 & 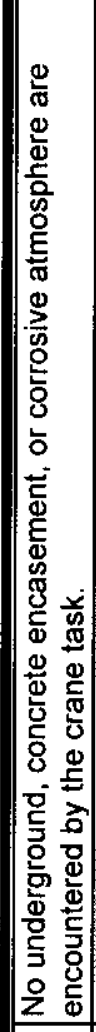 & 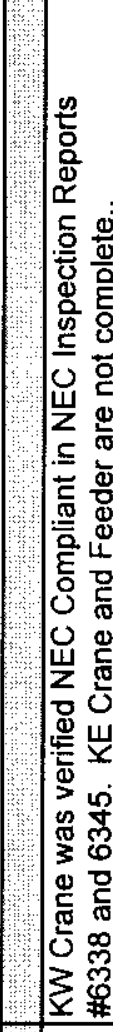 & & 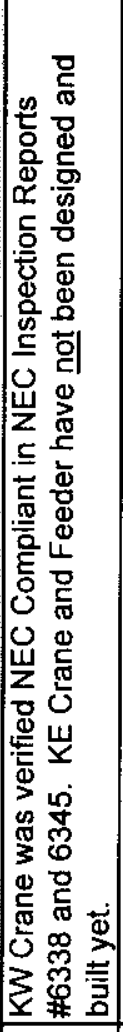 & 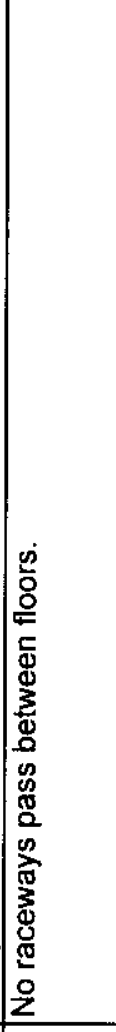 & & 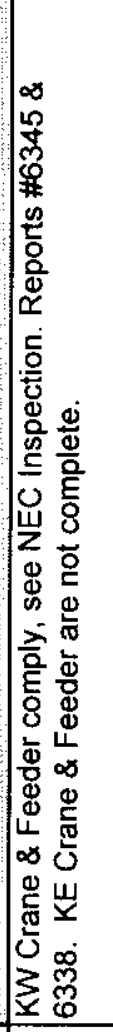 & 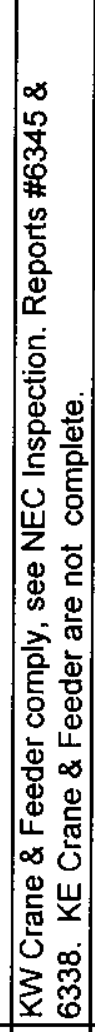 & 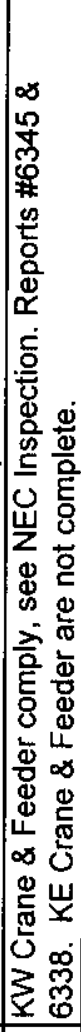 & 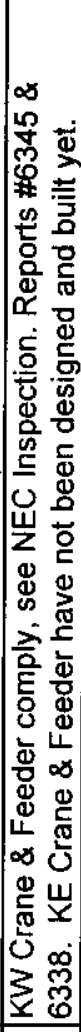 & 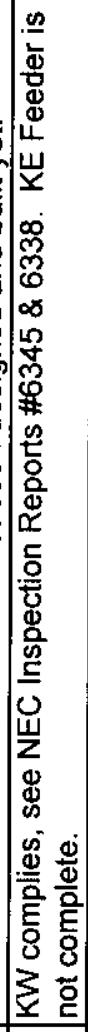 & 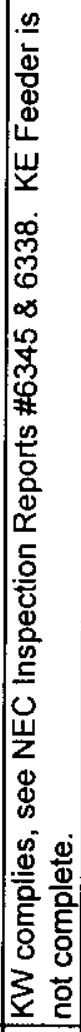 & 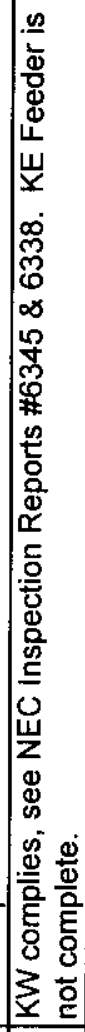 & 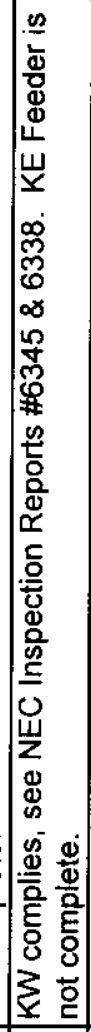 & 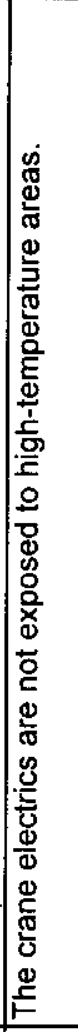 & 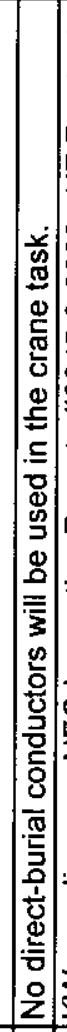 & 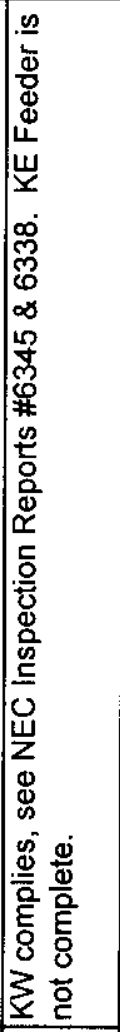 & & 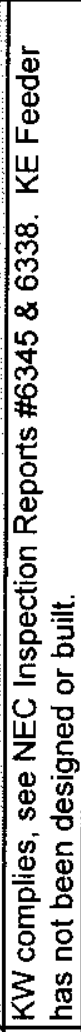 & 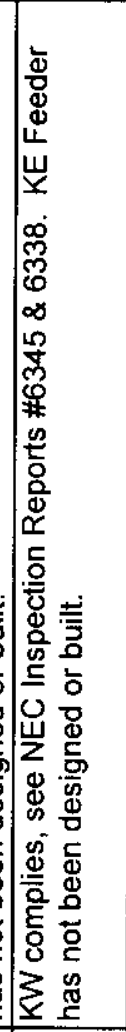 \\
\hline $\begin{array}{l}\text { 岕 } \\
\text { 心 } \\
\text { 心 }\end{array}$ & $\frac{\pi}{z}$ & 0 & & 0 & $\Sigma$ & & 10 & 0 & 0 & 0 & 0 & 0 & 0 & 0 & $\Sigma$ & 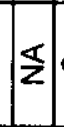 & 0 & & 0 & 0 \\
\hline 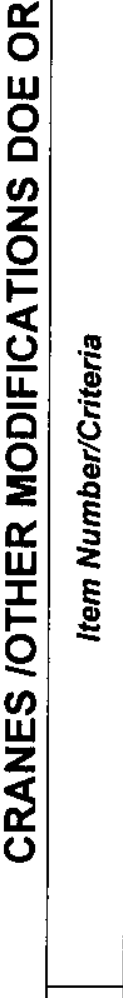 & 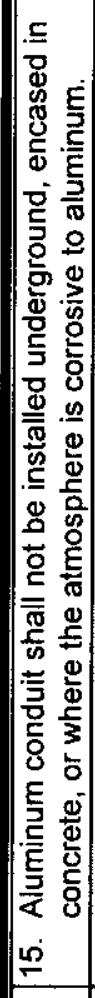 & 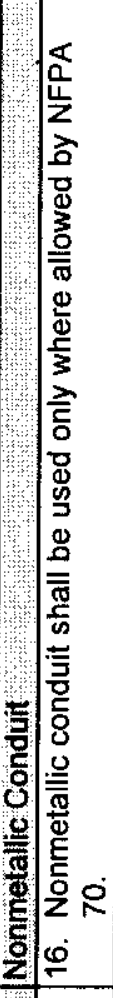 & 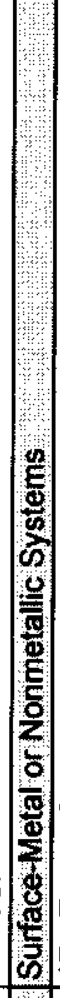 & 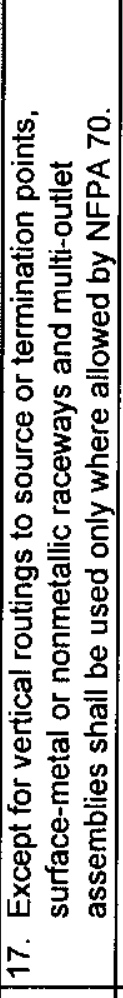 & 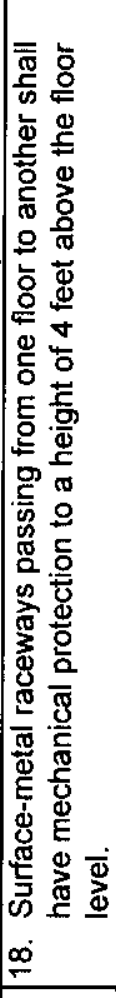 & 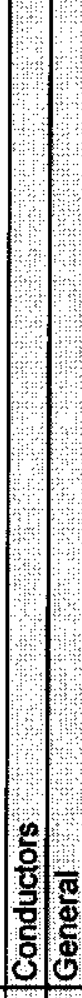 & 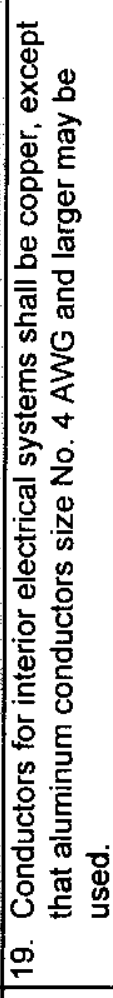 & 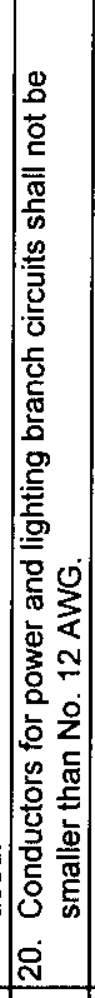 & 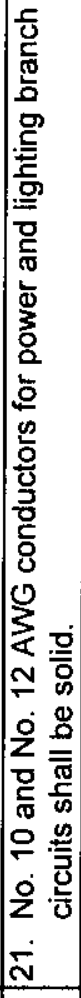 & 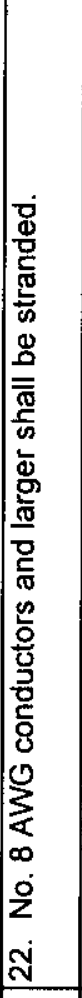 & 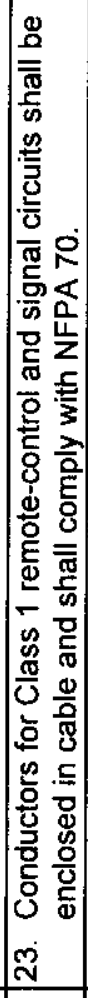 & 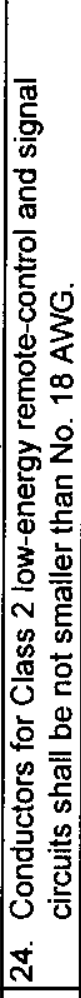 & 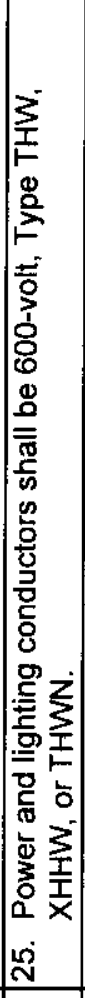 & 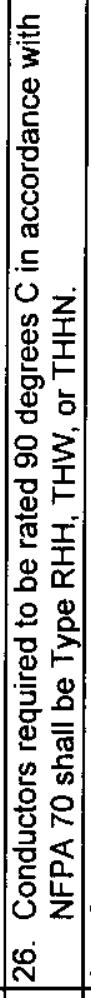 & 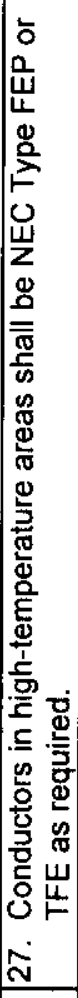 & 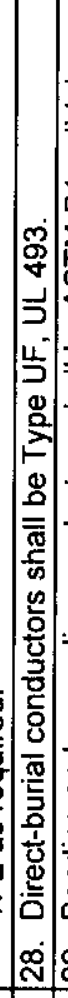 & 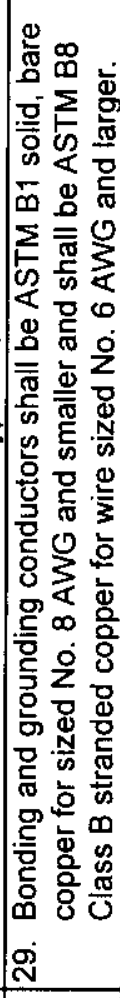 & 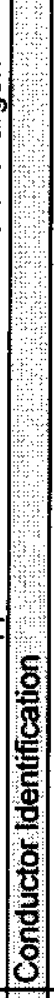 & 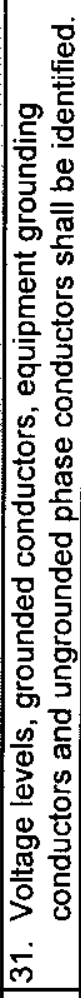 & 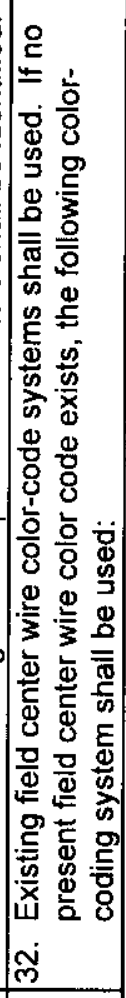 \\
\hline 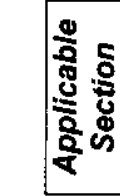 & 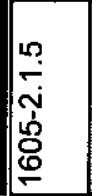 & 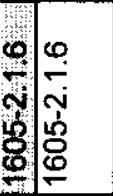 & 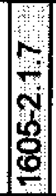 & 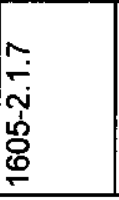 & 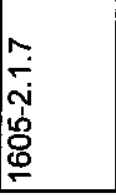 & & 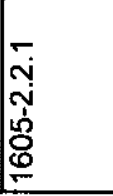 & ָ̃ & is & 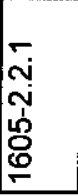 & & 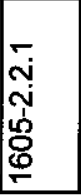 & 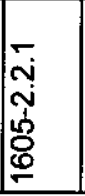 & 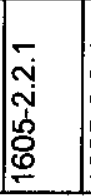 & 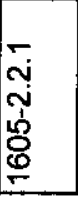 & & & & & 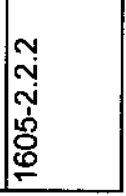 \\
\hline
\end{tabular}


SNF-4775, Rev. 2

Project A.5/A.6

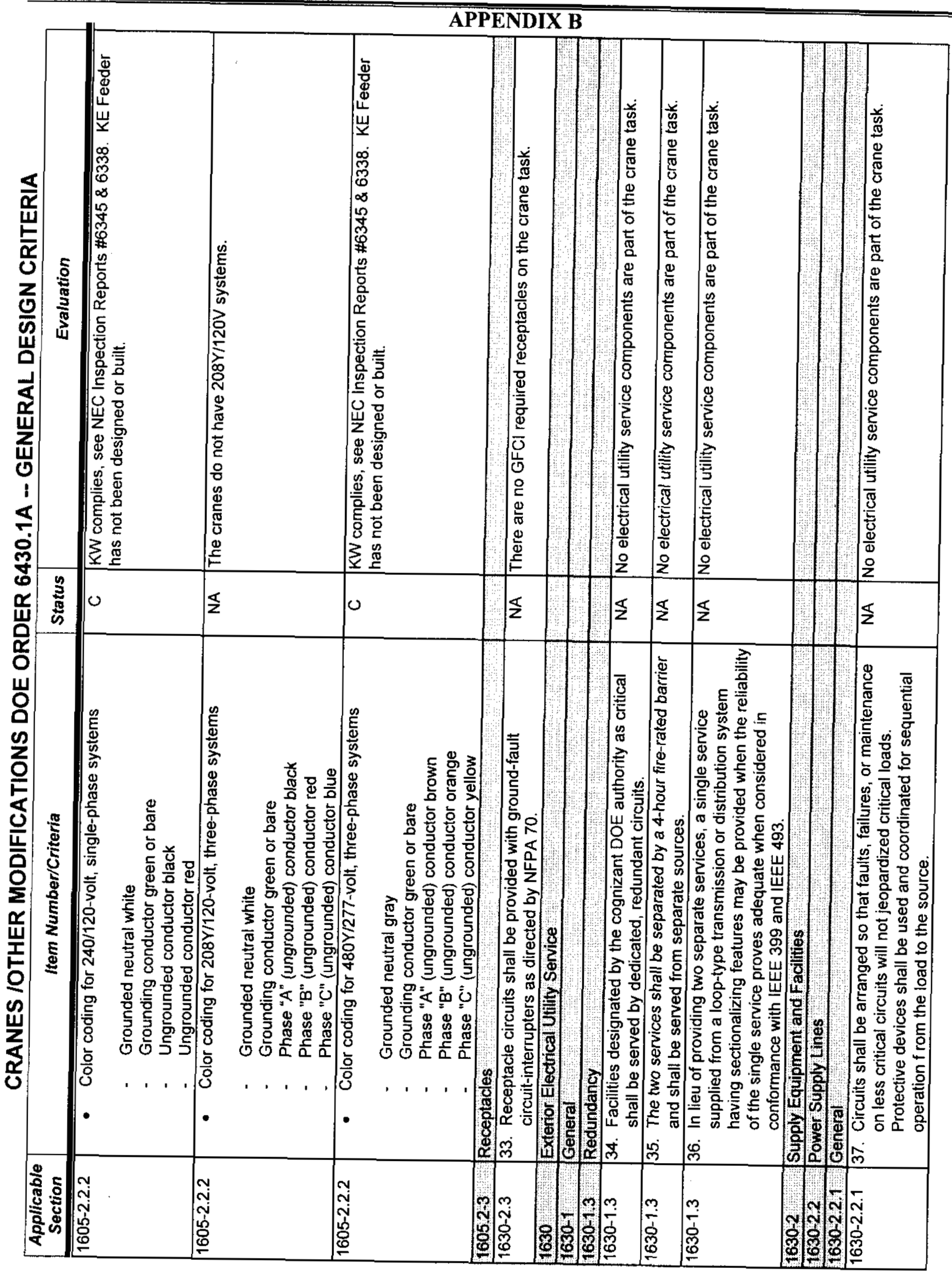

Page B-14 
SNF-4775, Rev. 2

Project A.5/A.6

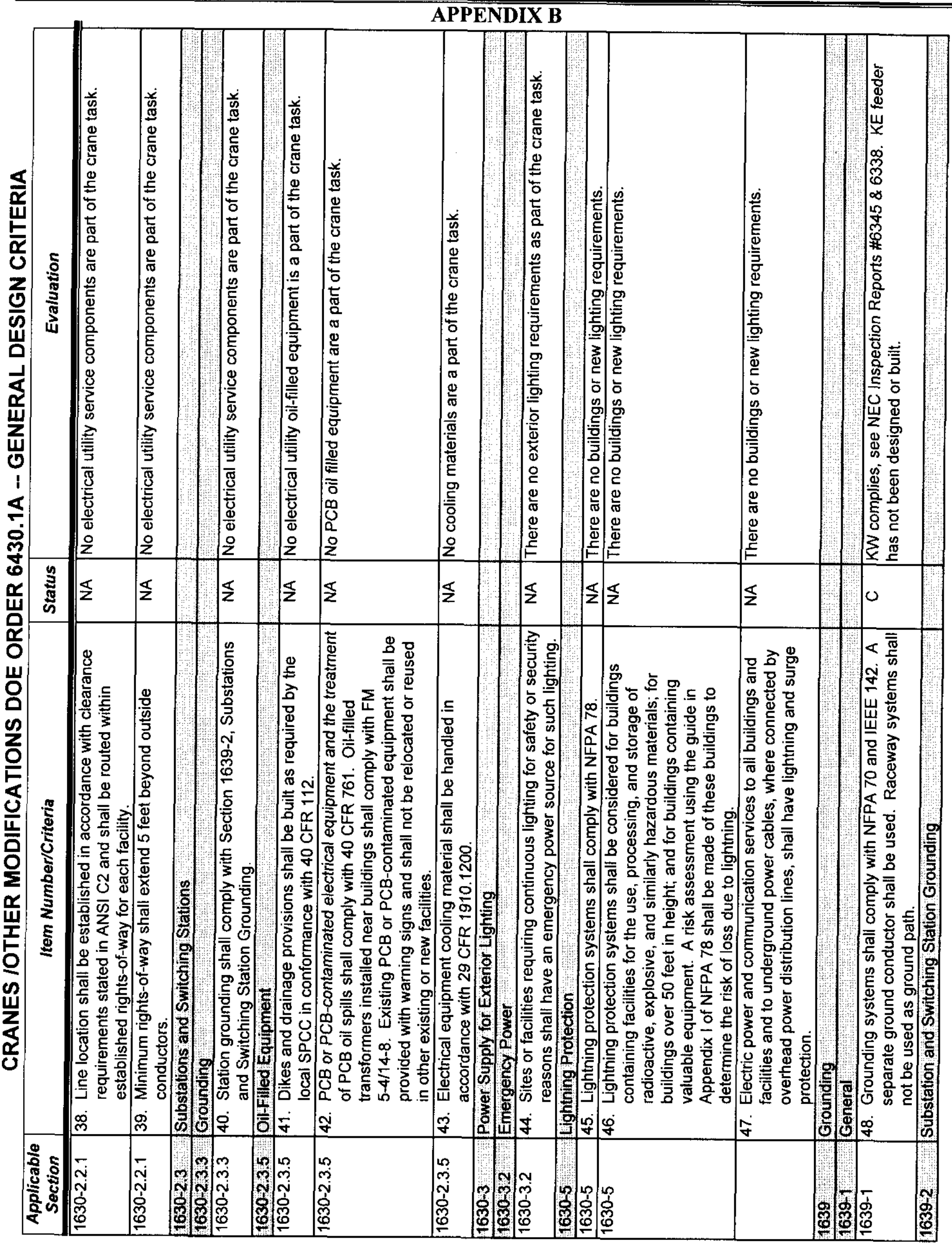

Page B-15 
SNF-4775, Rev. 2

Project A.5/A.6

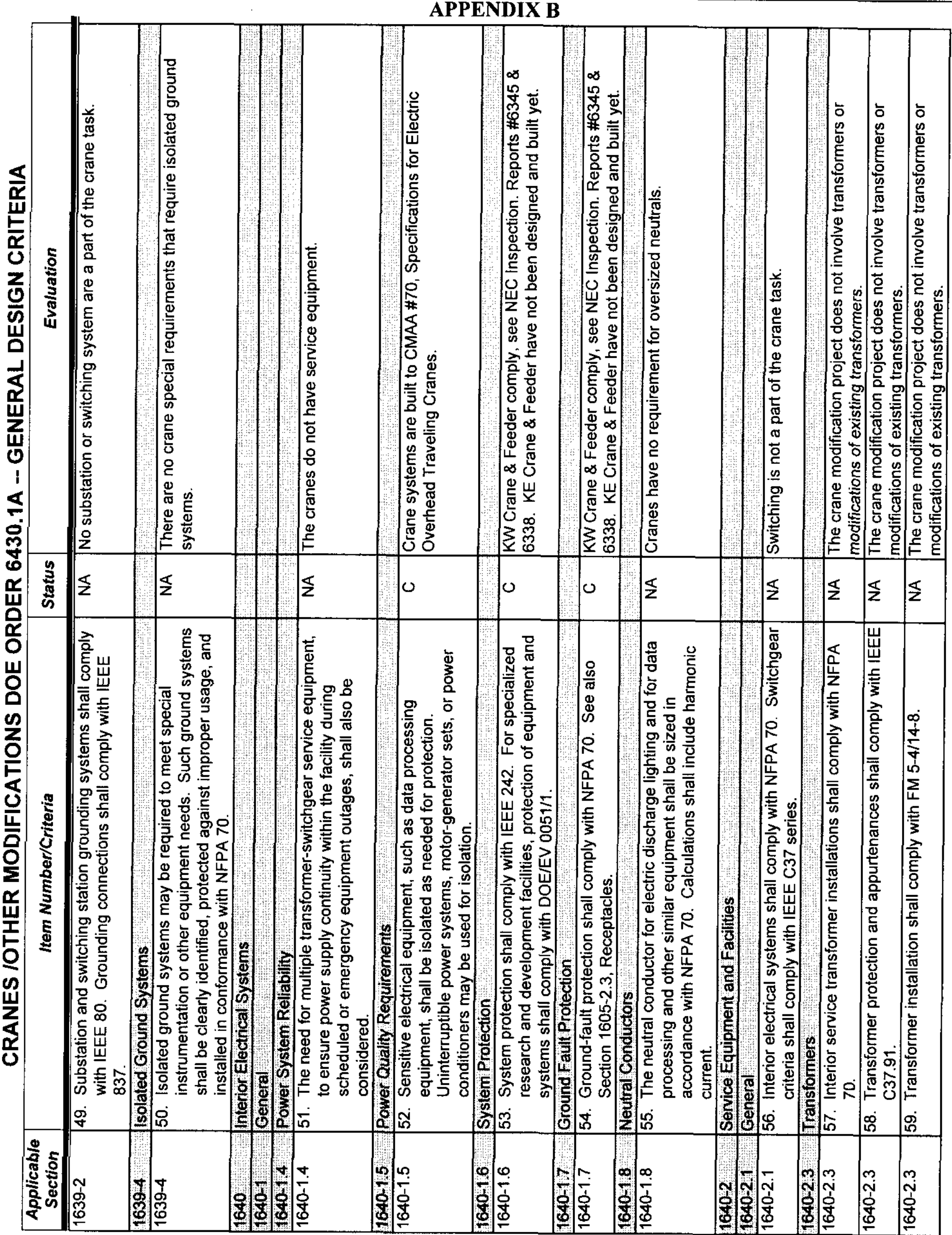


SNF-4775, Rev. 2

Project A.5/A.6

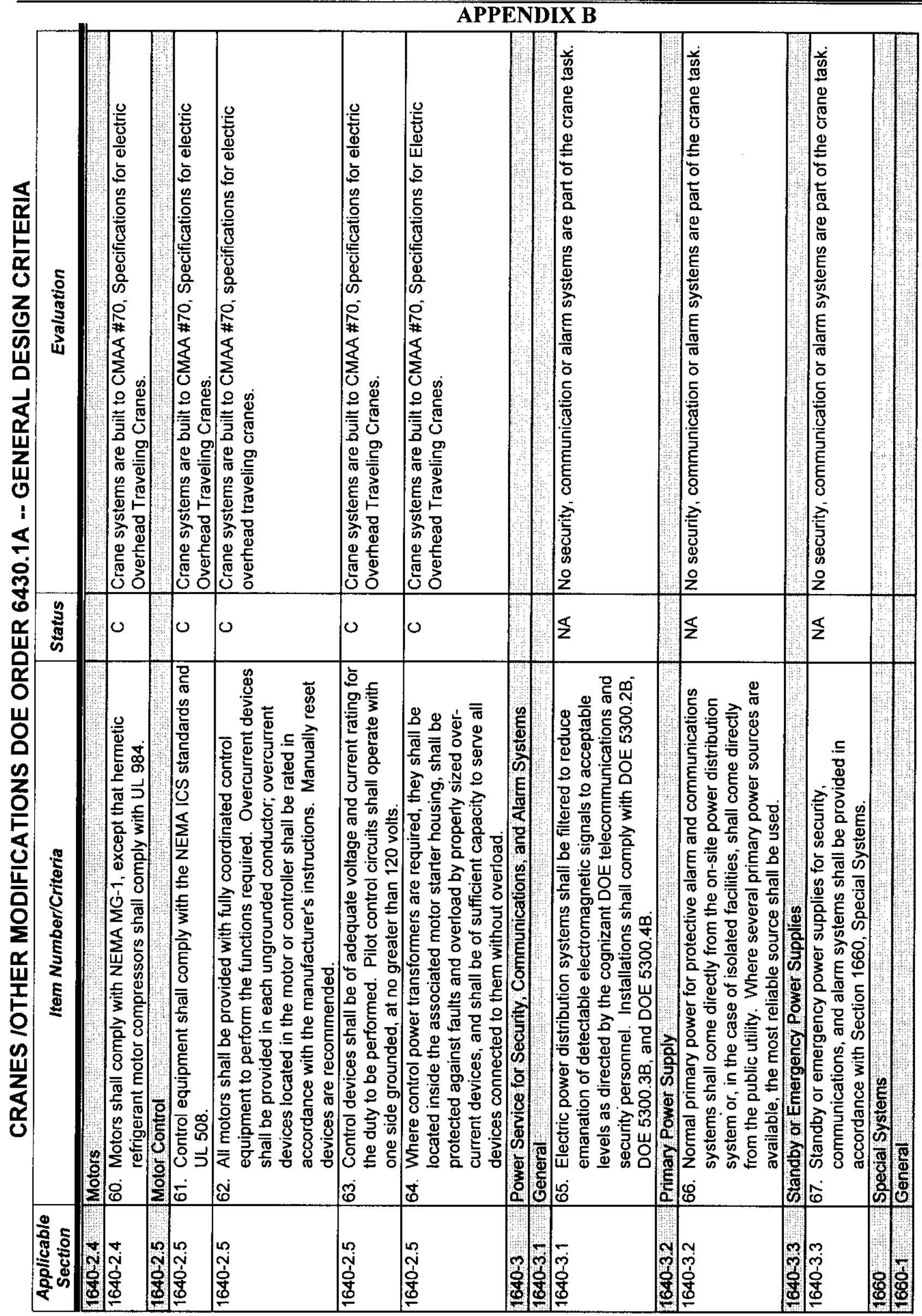




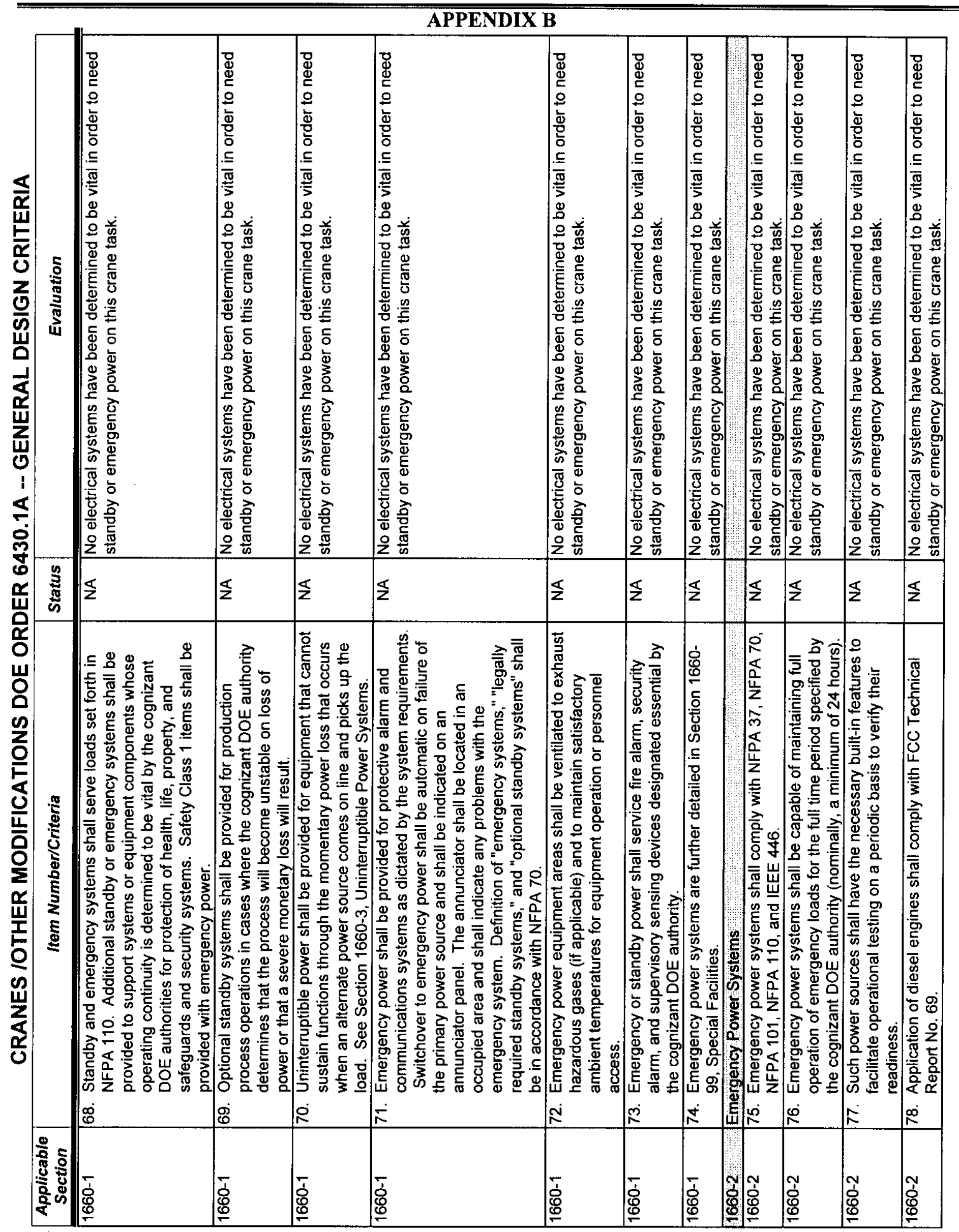




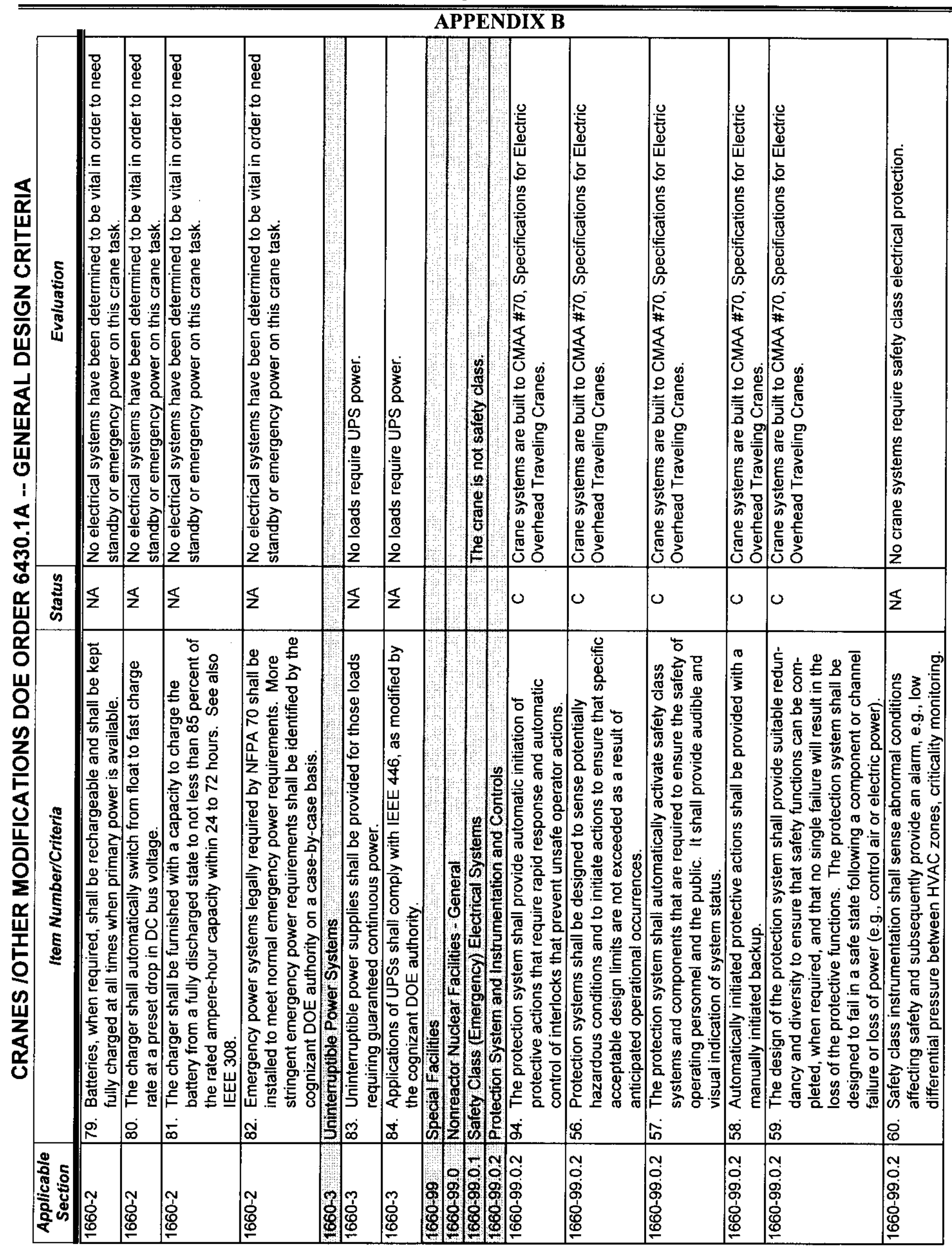


SNF-4775, Rev. 2

Project A.5/A.6

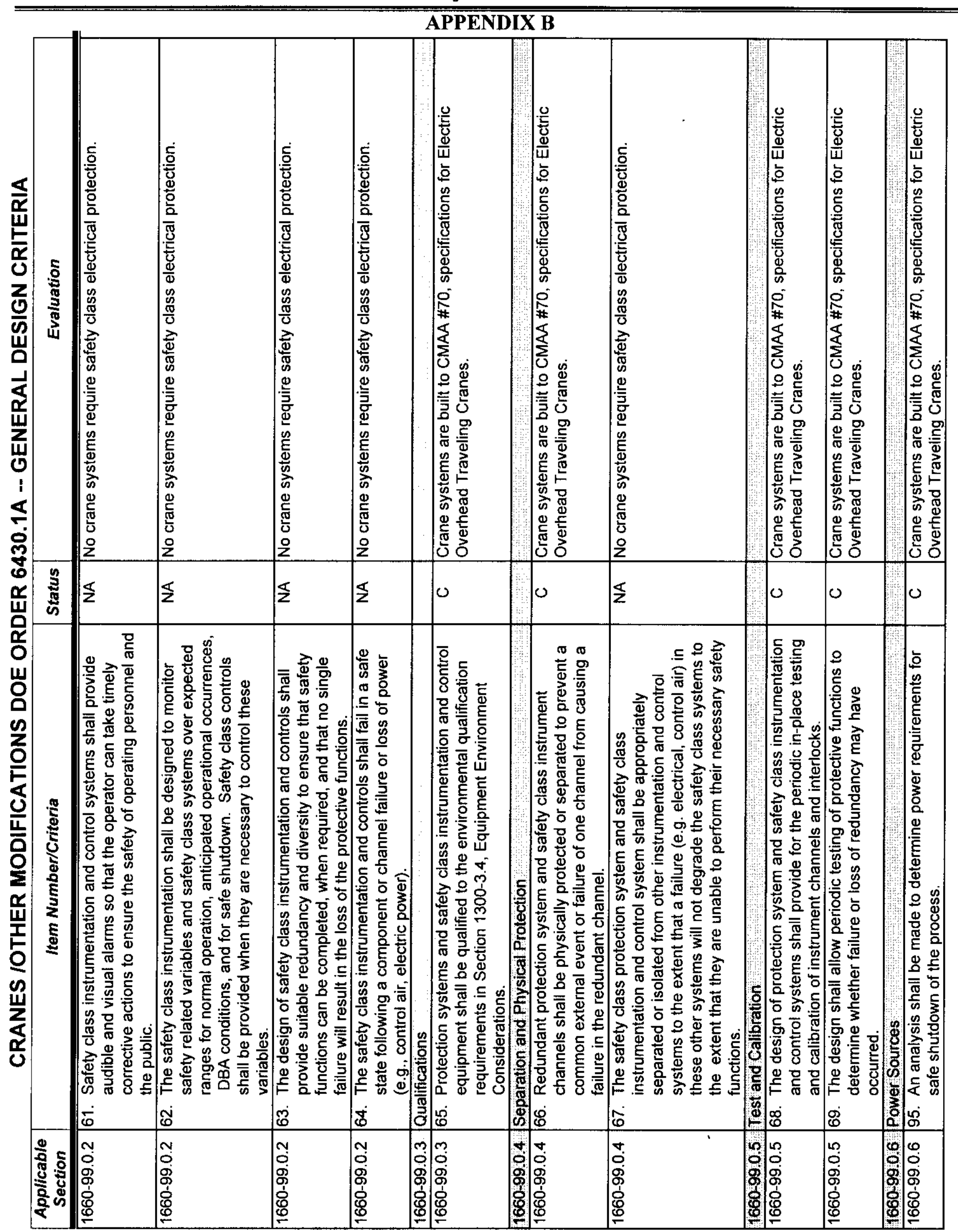




\begin{tabular}{|c|c|c|c|c|c|c|c|c|c|c|c|}
\hline \multicolumn{12}{|c|}{ APPENDIX B } \\
\hline 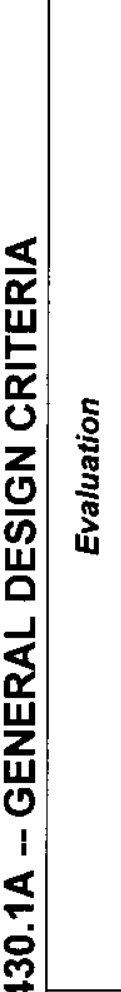 & 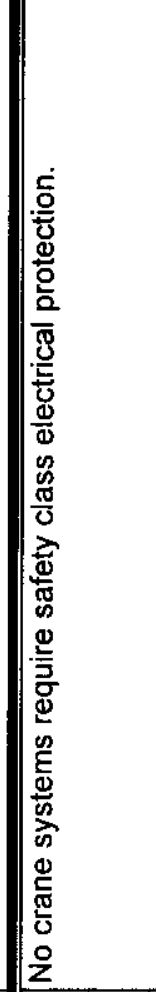 & 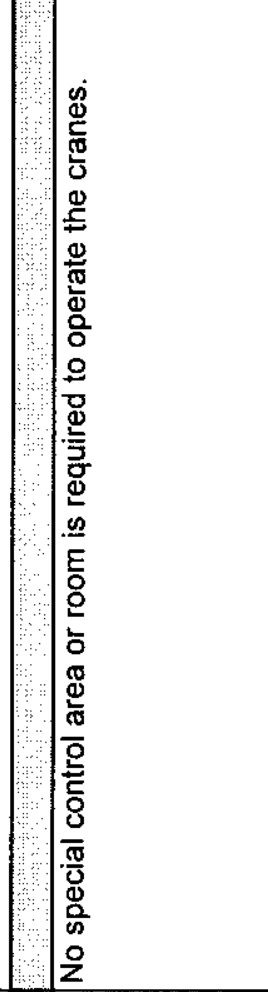 & 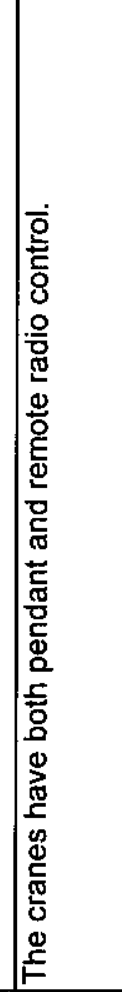 & & 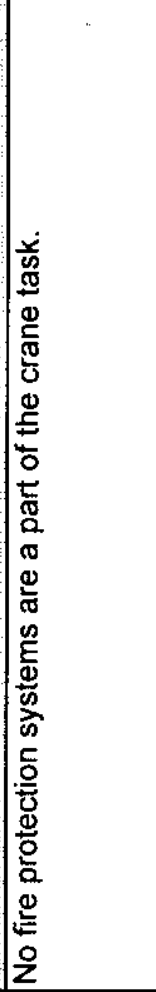 & 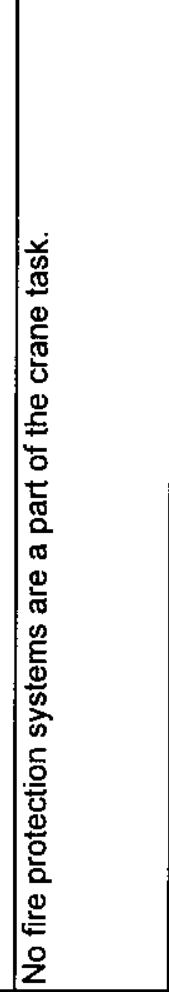 & 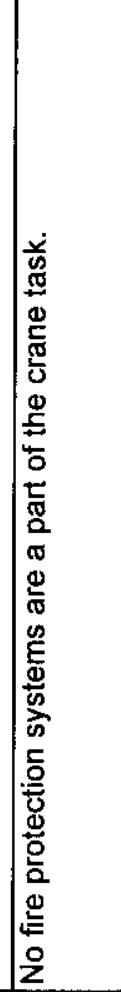 & 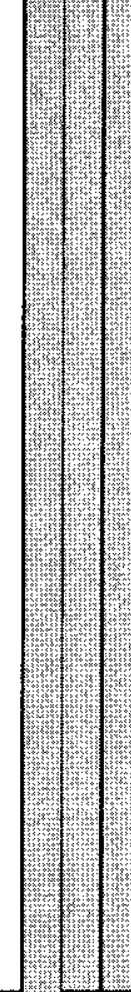 & 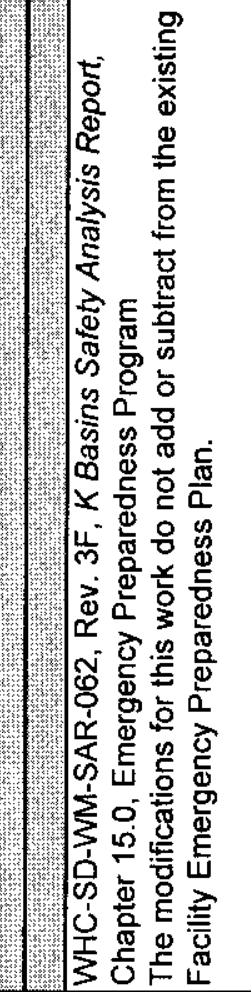 & 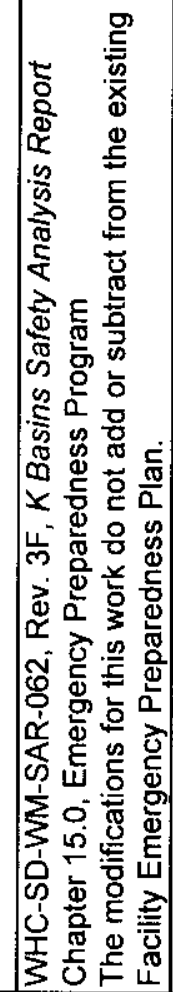 & 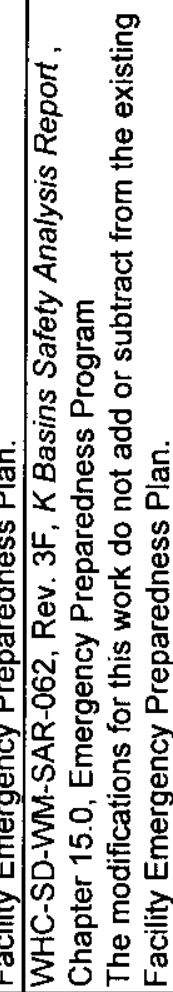 \\
\hline 苞 & $\S$ & $\frac{\mathbb{s}}{2}$ & 0 & & $\Sigma$ & $\S$ & $\frac{\pi}{z}$ & & $\frac{5}{2}$ & $\S$ & $\frac{5}{2}$ \\
\hline 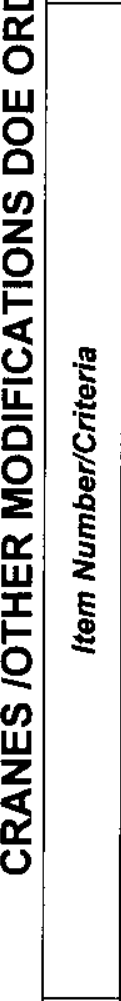 & 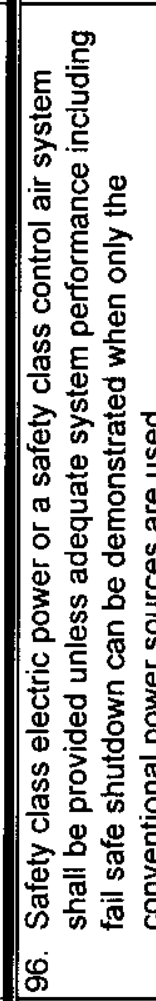 & 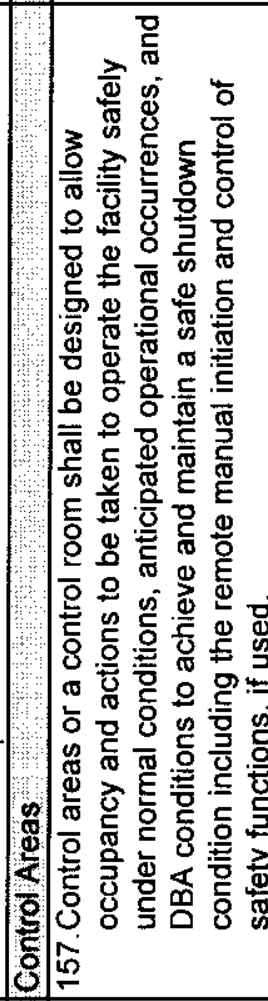 & 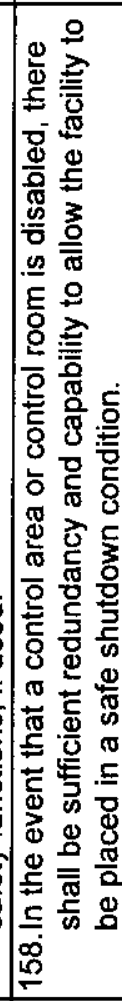 & 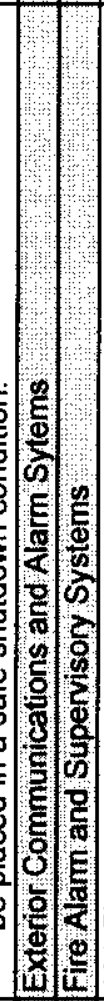 & 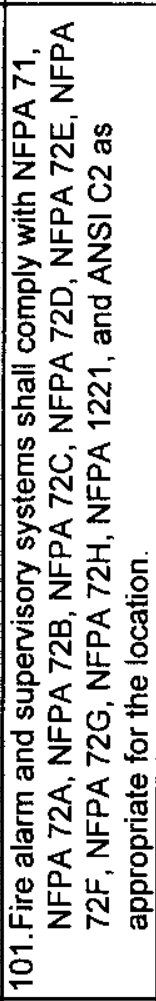 & 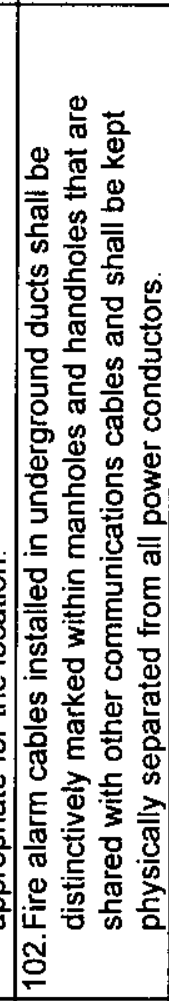 & 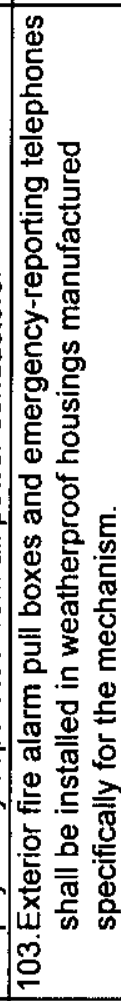 & 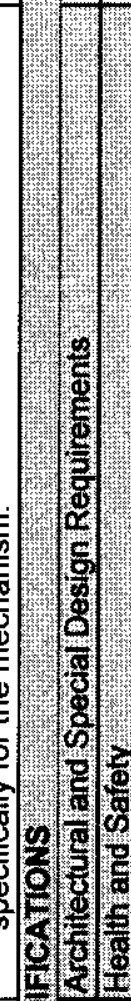 & 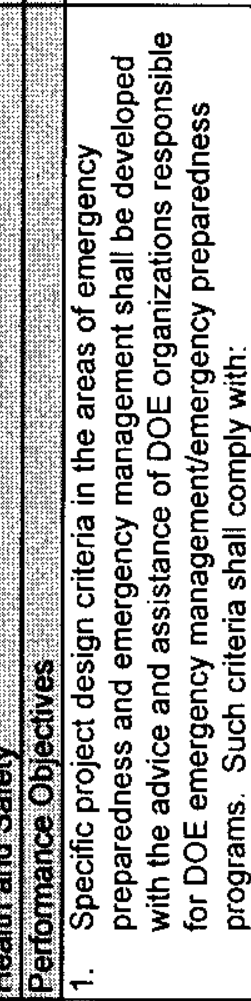 & $\begin{array}{l}\mid \leq \\
\vdots \\
0 \\
0 \\
0 \\
0 \\
0 \\
0 \\
0\end{array}$ & 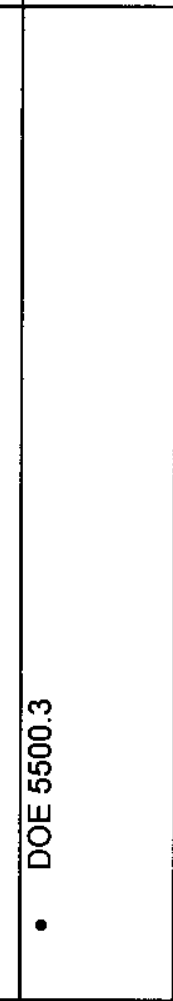 \\
\hline 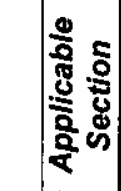 & $\begin{array}{l}0 \\
0 \\
0 \\
8 \\
0 \\
0 \\
0 \\
0 \\
0\end{array}$ & 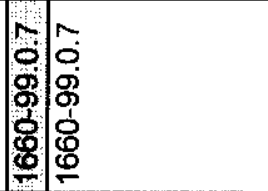 & 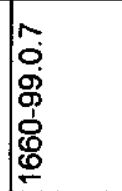 & 웡융 & & $F$ & 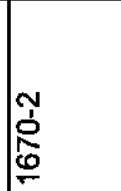 & 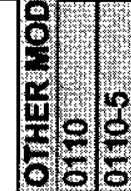 & 5 & 离 & | \\
\hline
\end{tabular}


SNF-4775, Rev. 2

Project A.5/A.6

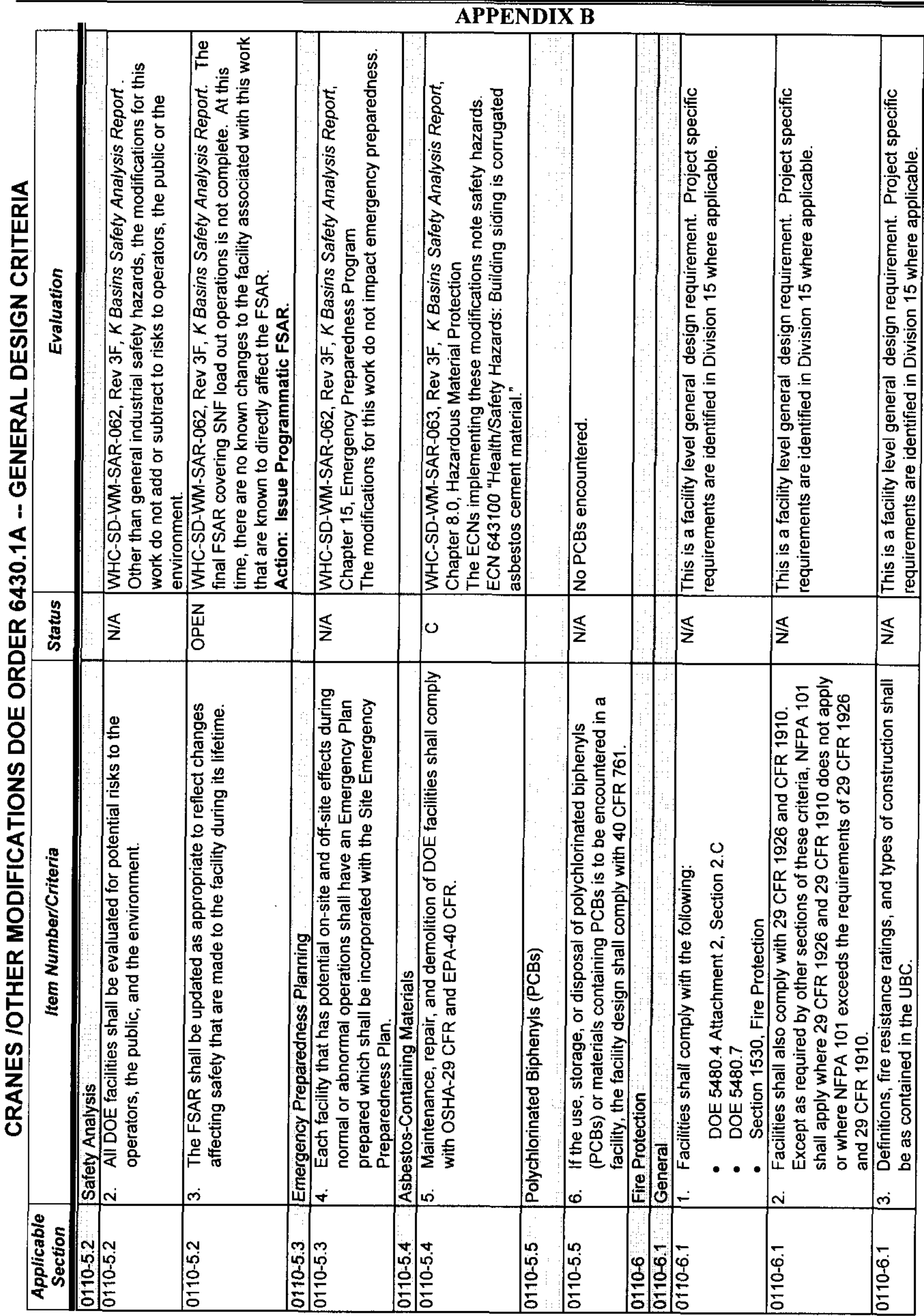


SNF-4775, Rev. 2

Project A.5/A.6

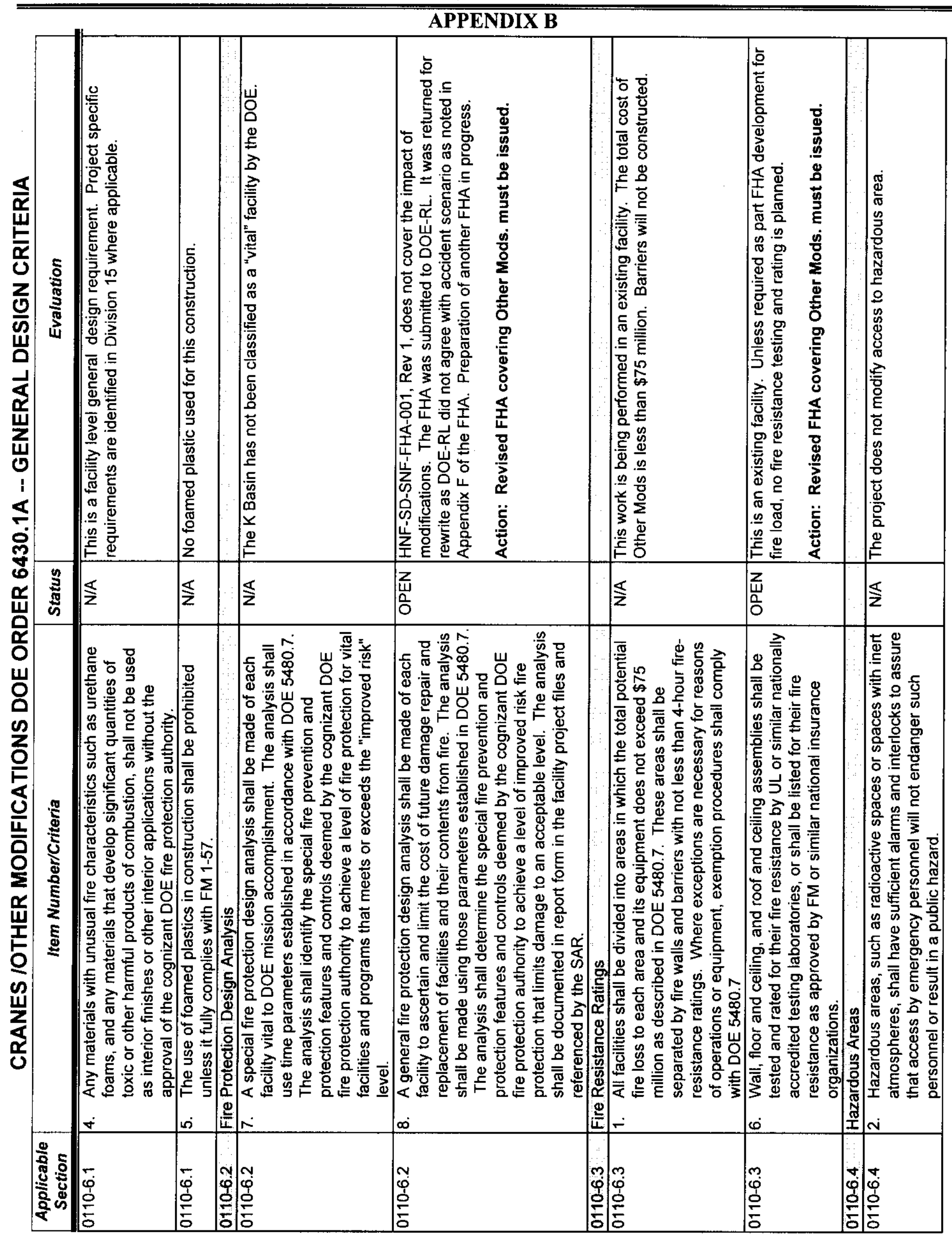




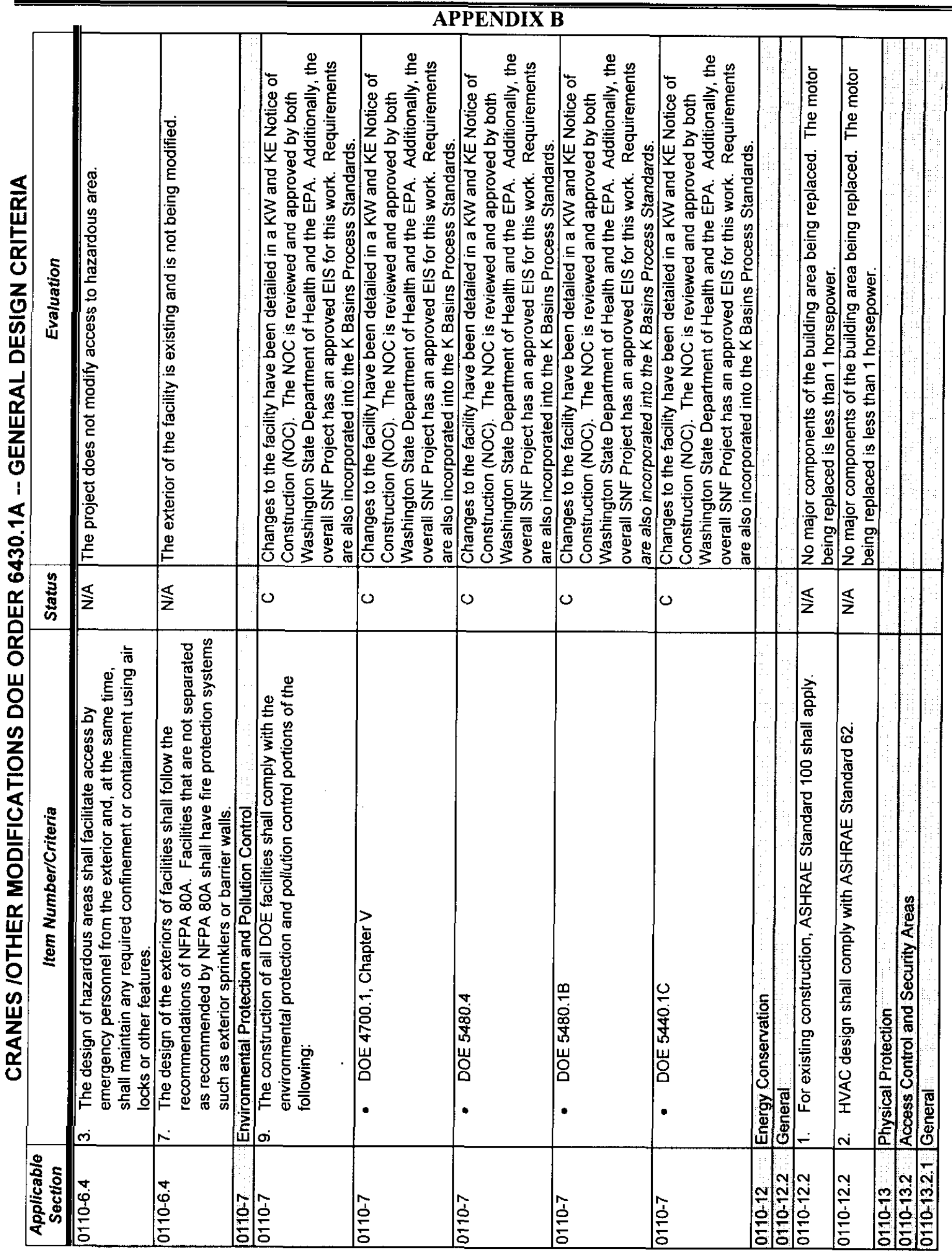




\begin{tabular}{|c|c|c|c|c|c|c|c|c|c|c|c|c|c|c|c|c|}
\hline \multirow{2}{*}{\multicolumn{2}{|c|}{ 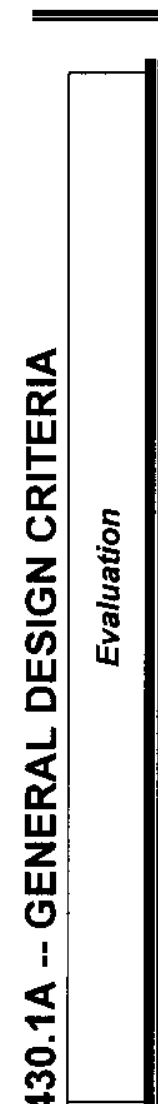 }} & \multicolumn{15}{|c|}{ APPENDIX B } \\
\hline & & 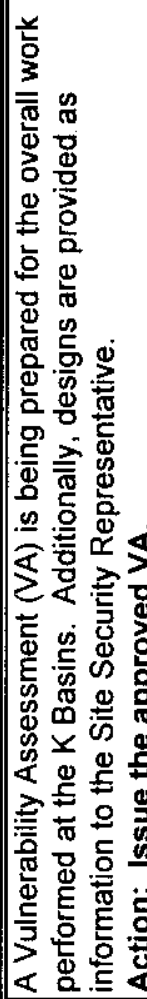 & 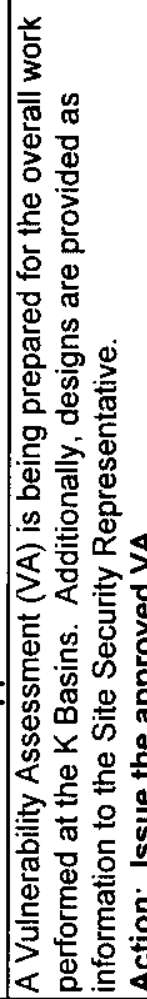 & 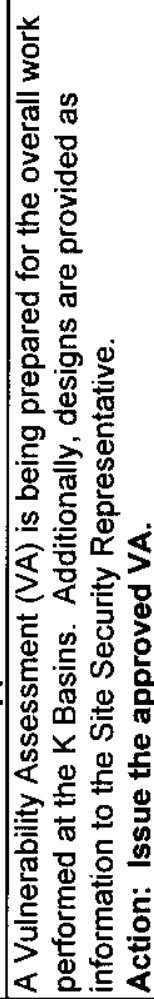 & 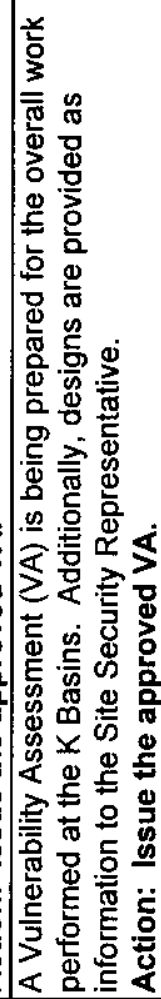 & & 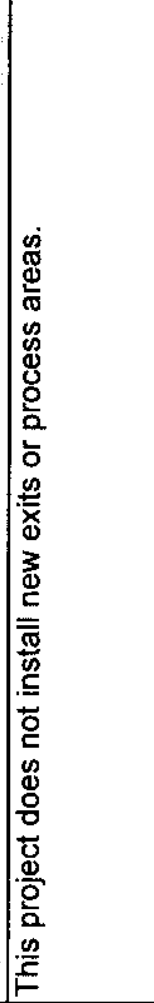 & 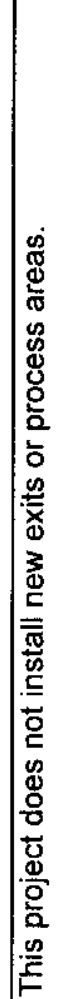 & 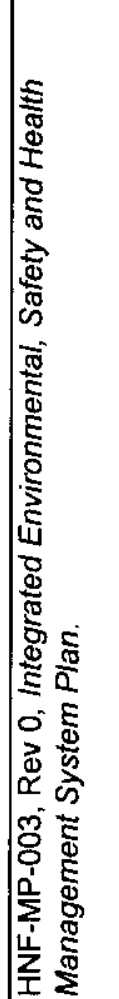 & 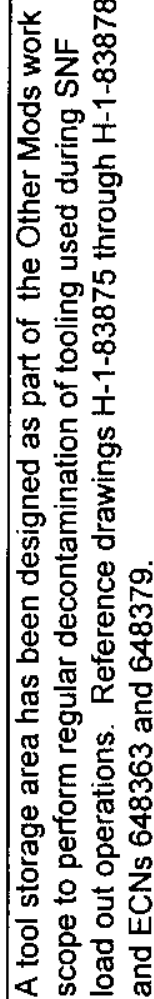 & & 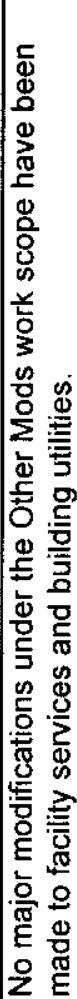 & 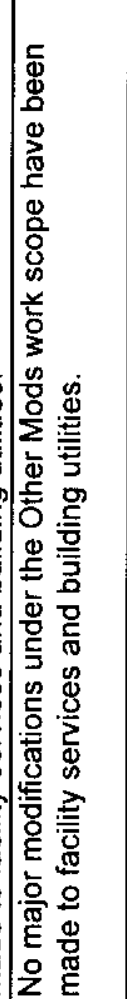 & 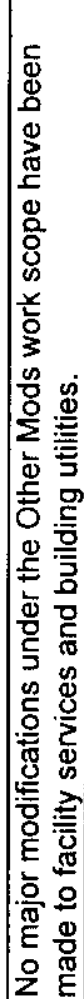 & 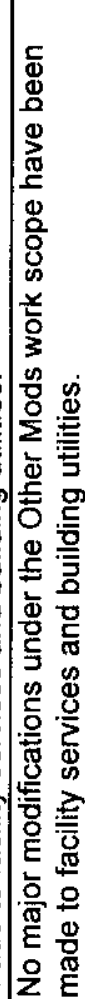 & 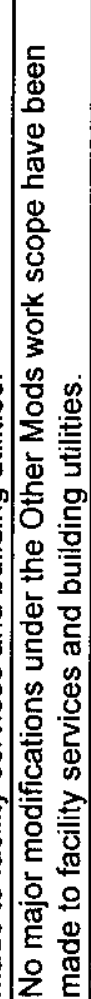 \\
\hline $\bar{\alpha}$ & 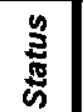 & 衤 & $\begin{array}{l}\text { 능 } \\
\text { ó }\end{array}$ & 立 & $\begin{array}{l}\text { zu } \\
\text { o } \\
\end{array}$ & & $\$$ & $\frac{\mathbb{Z}}{\mathbf{z}}$ & 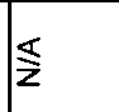 & 0 & & 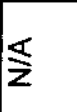 & $\mathbf{z}$ & $\frac{\$}{z}$ & 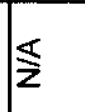 & $\frac{\$}{z}$ \\
\hline 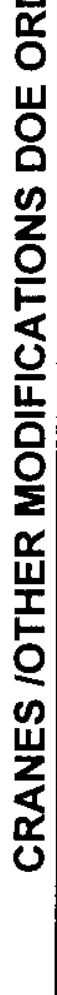 & 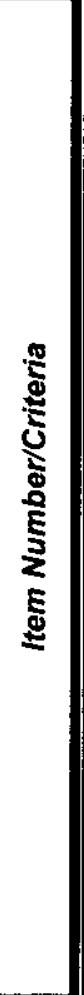 & 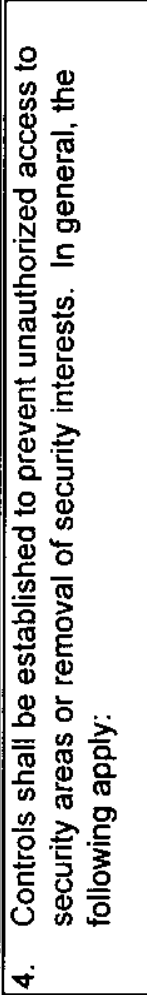 & 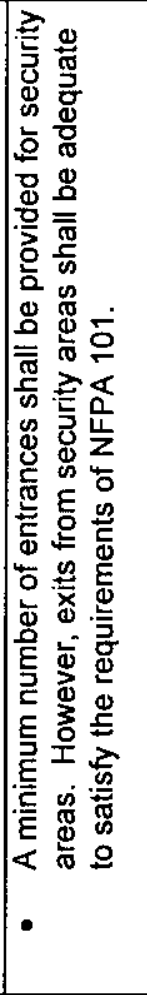 & 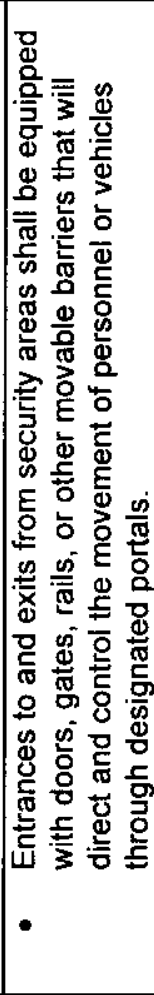 & 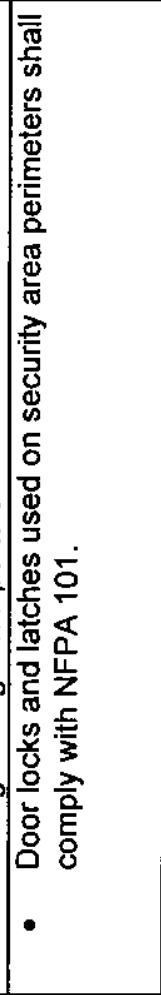 & 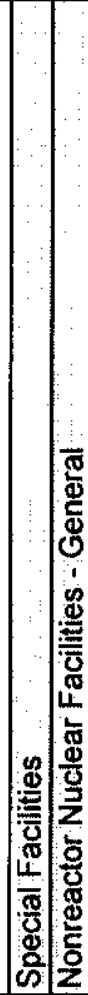 & 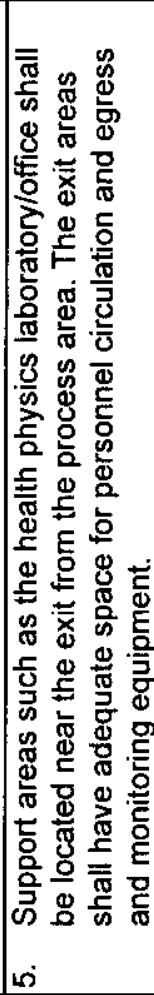 & 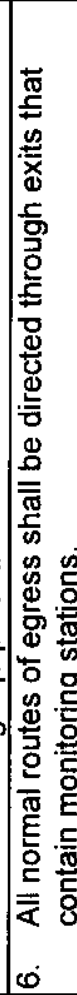 & 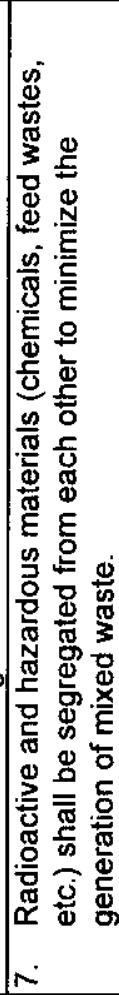 & 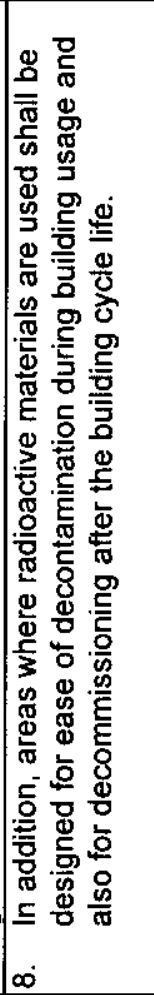 & 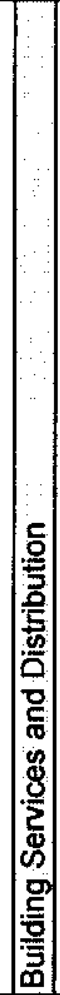 & 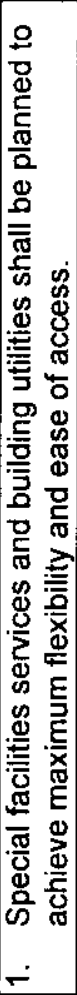 & 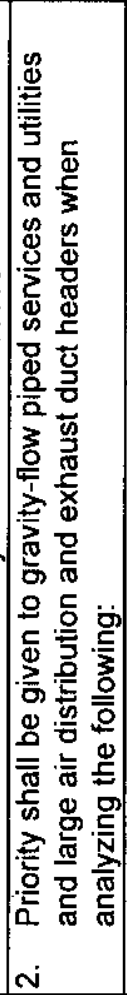 & 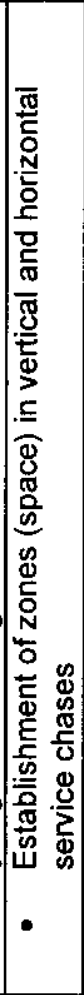 & 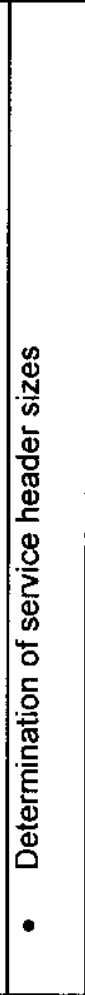 & 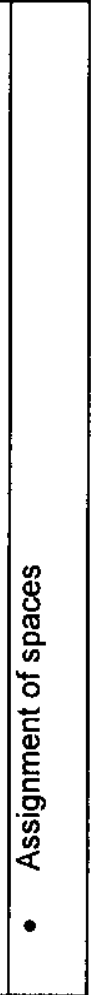 \\
\hline & 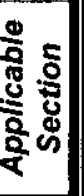 & $\frac{1}{0}$ & & 空 & 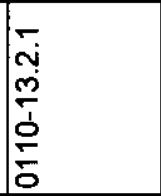 & 官 & & 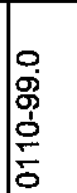 & 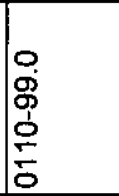 & 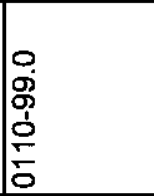 & & & 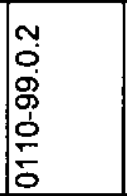 & 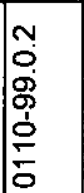 & 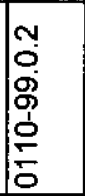 & 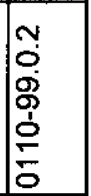 \\
\hline
\end{tabular}


SNF-4775, Rev. 2

Project A.5/A.6

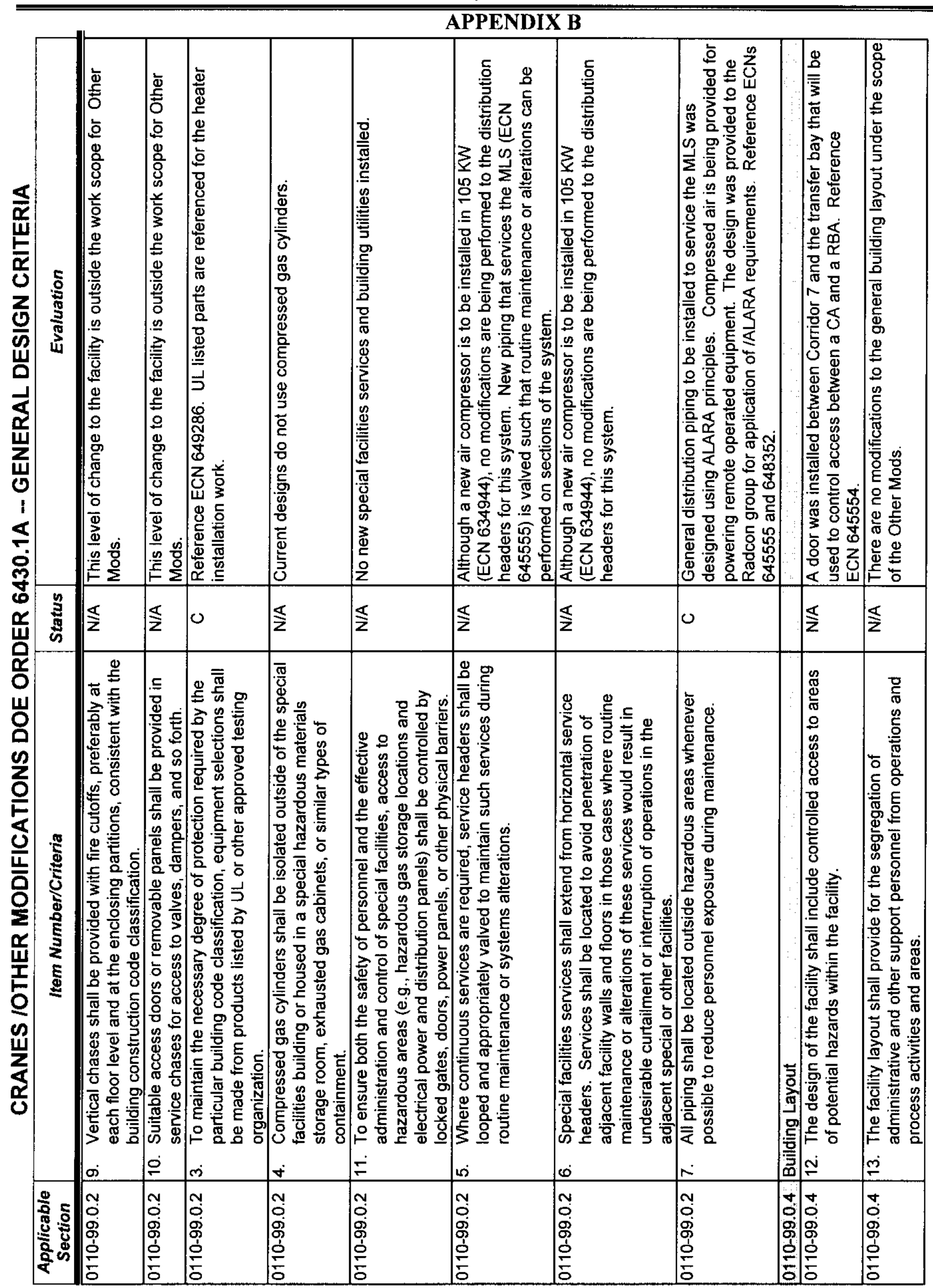


SNF-4775, Rev. 2

Project A.5/A.6

\begin{tabular}{|c|c|c|c|c|c|c|c|c|c|c|c|}
\hline \multirow{2}{*}{\multicolumn{2}{|c|}{ 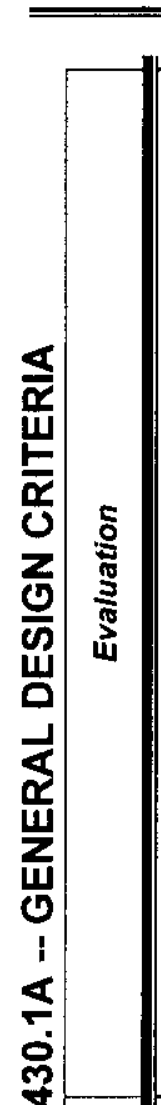 }} & \multicolumn{10}{|c|}{ APPENDIX B } \\
\hline & & 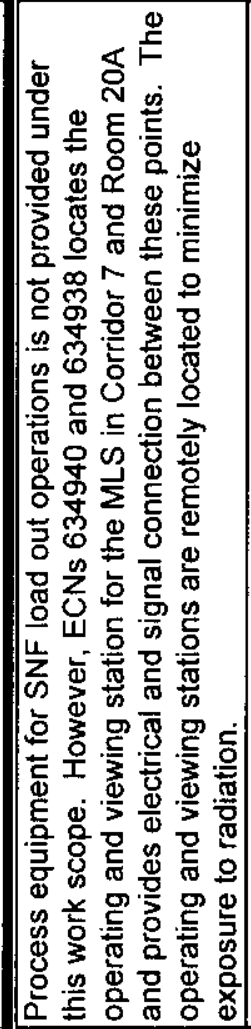 & 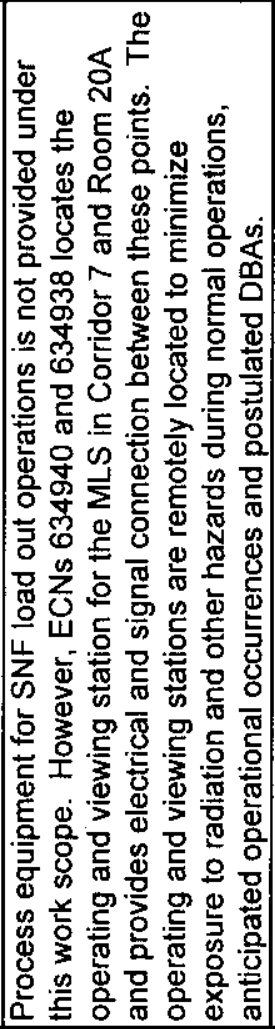 & 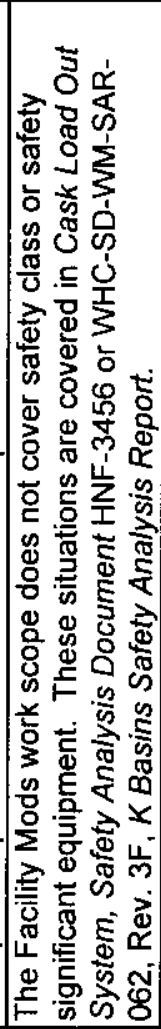 & 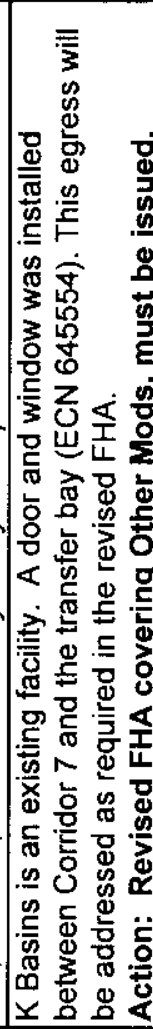 & 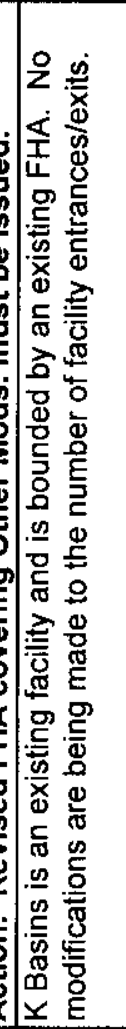 & 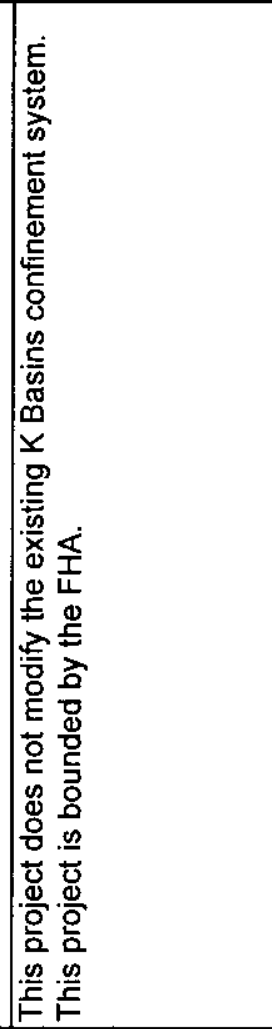 & 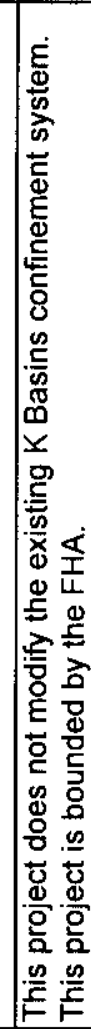 & 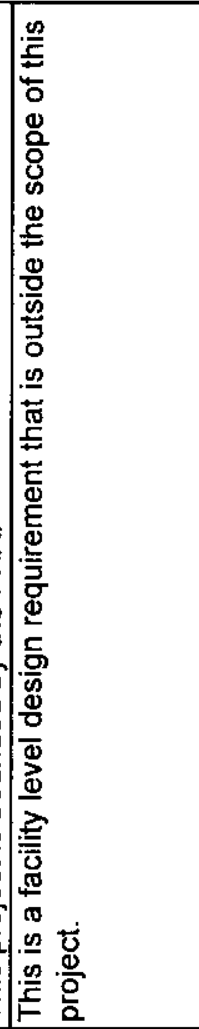 & 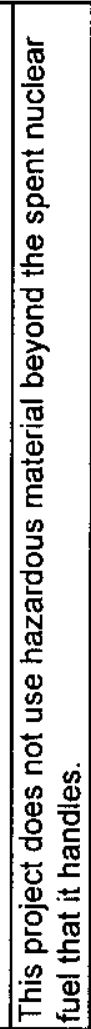 & 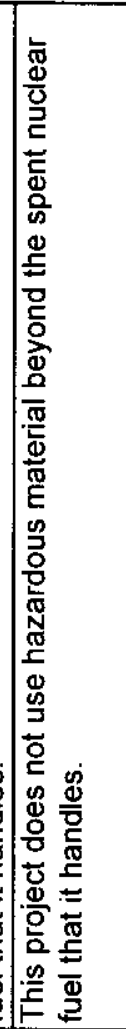 \\
\hline $\boldsymbol{\alpha}$ & 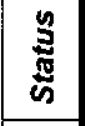 & $\| 0$ & 0 & $\$ \$$ & 立 & $\$$ & $\stackrel{5}{z}$ & $\frac{\varsigma}{z}$ & $\stackrel{\mathbb{z}}{\mathbf{z}}$ & $\stackrel{2}{z}$ & 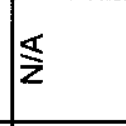 \\
\hline 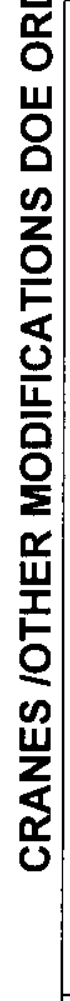 & 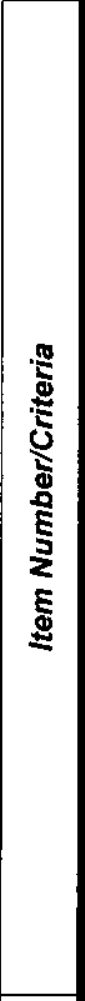 & 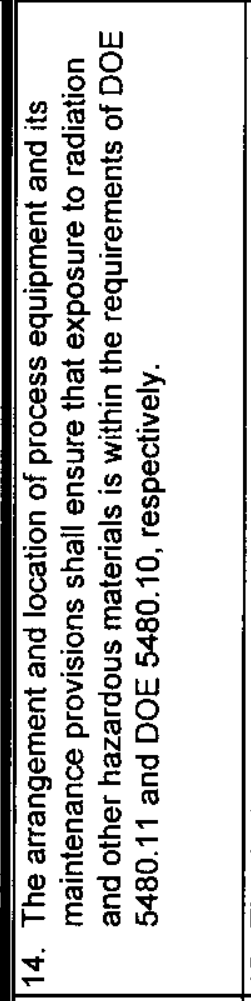 & 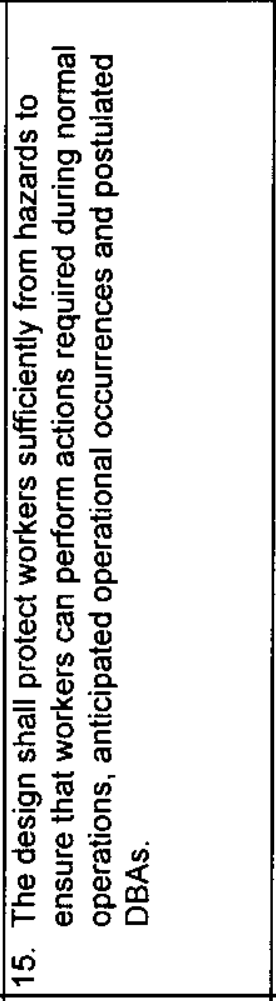 & 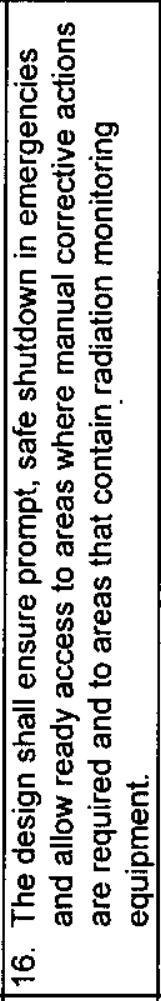 & 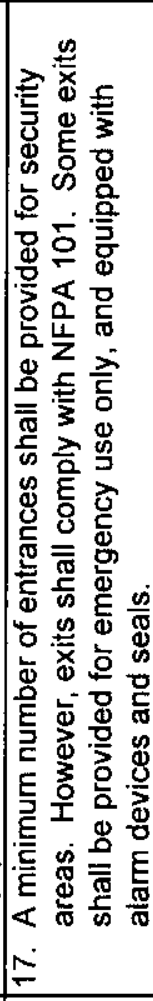 & 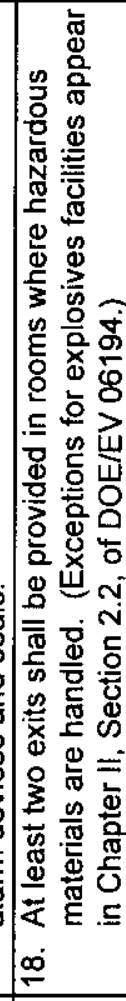 & 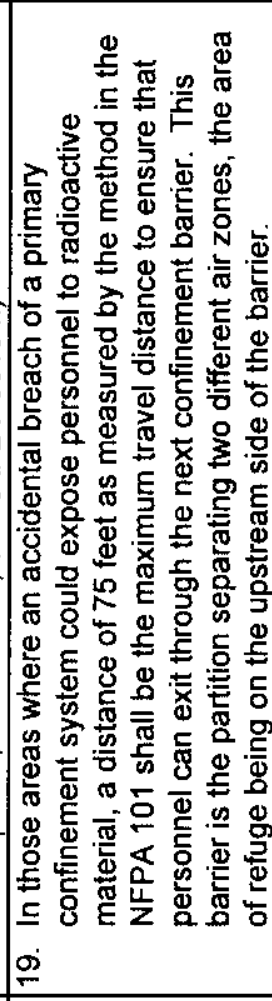 & 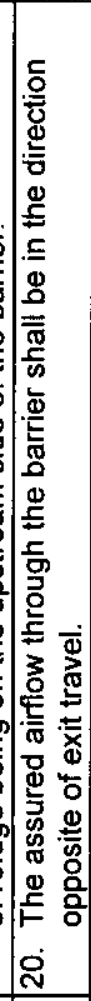 & 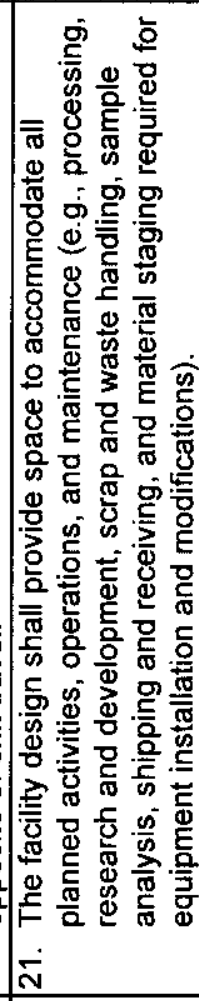 & 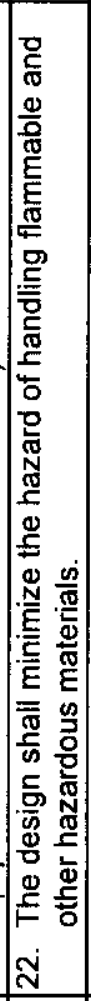 & 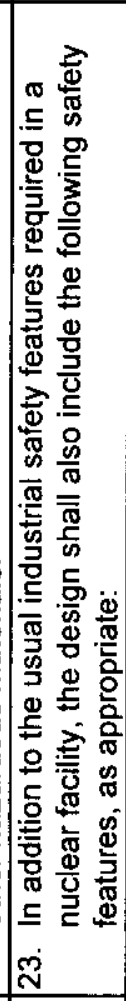 \\
\hline & $\begin{array}{l}\frac{0}{0} \\
\frac{0}{8} \\
\frac{0}{2} \\
\frac{2}{2} \\
\frac{2}{2}\end{array}$ & 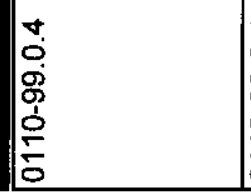 & 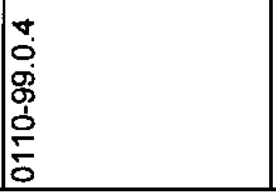 & 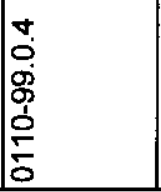 & 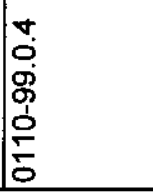 & 10 & 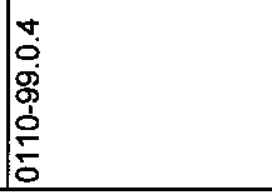 & 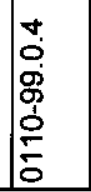 & $\begin{array}{l}\text { o } \\
0 \\
0 \\
0 \\
0\end{array}$ & 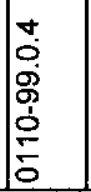 & 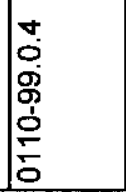 \\
\hline
\end{tabular}


SNF-4775, Rev. 2

Project A.5/A.6

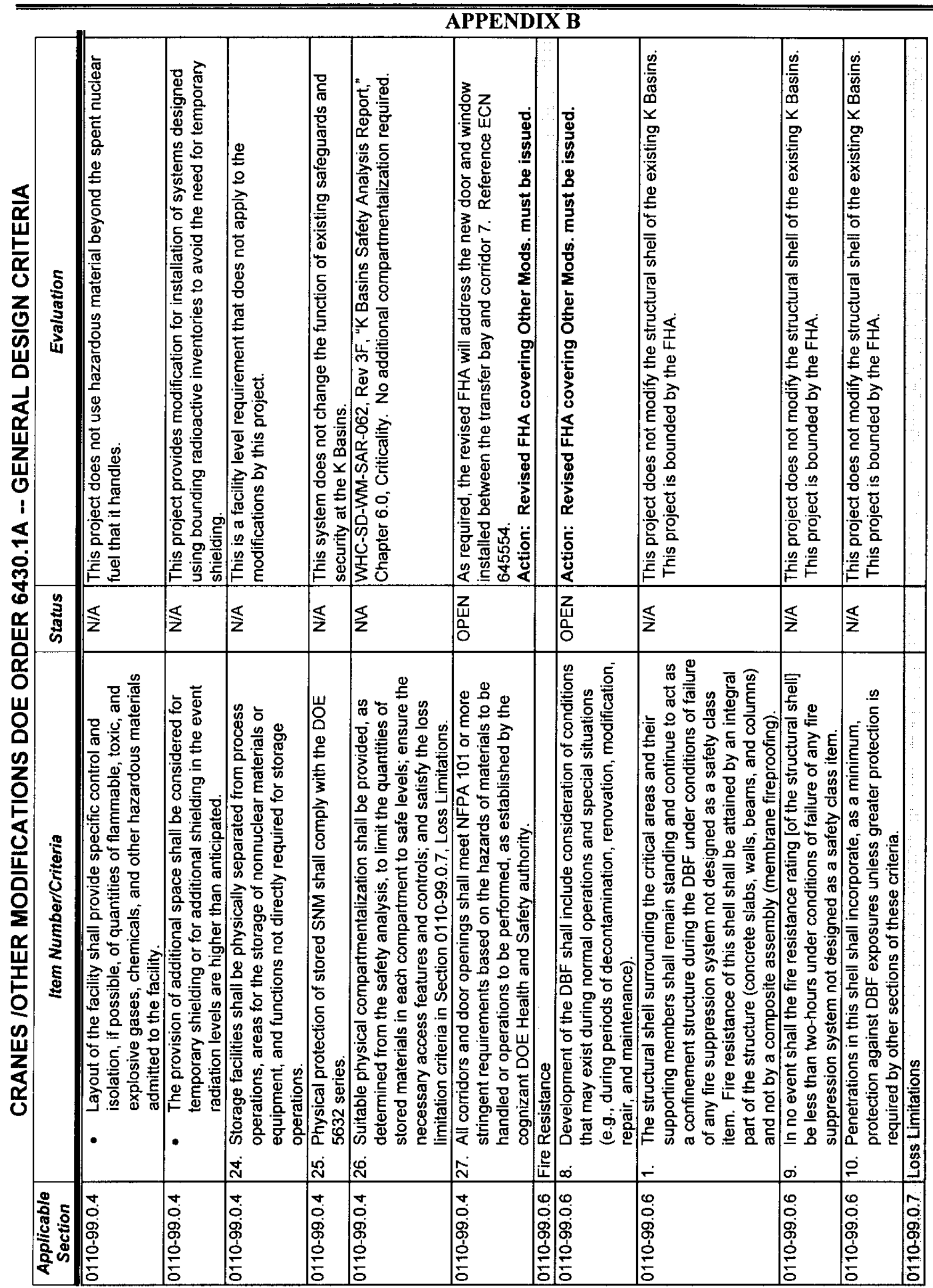


SNF-4775, Rev. 2

Project A.5/A.6

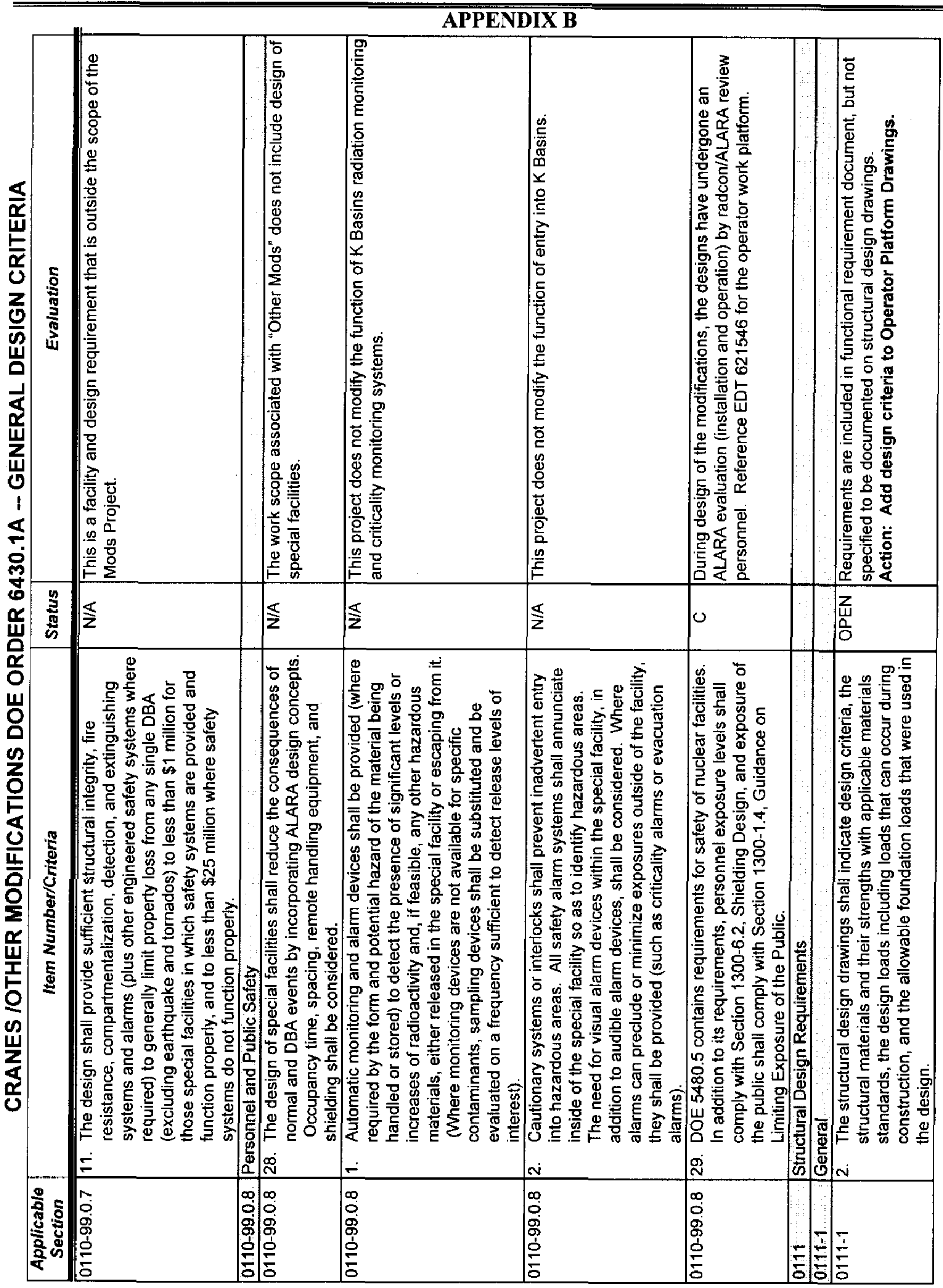

Page B-29 


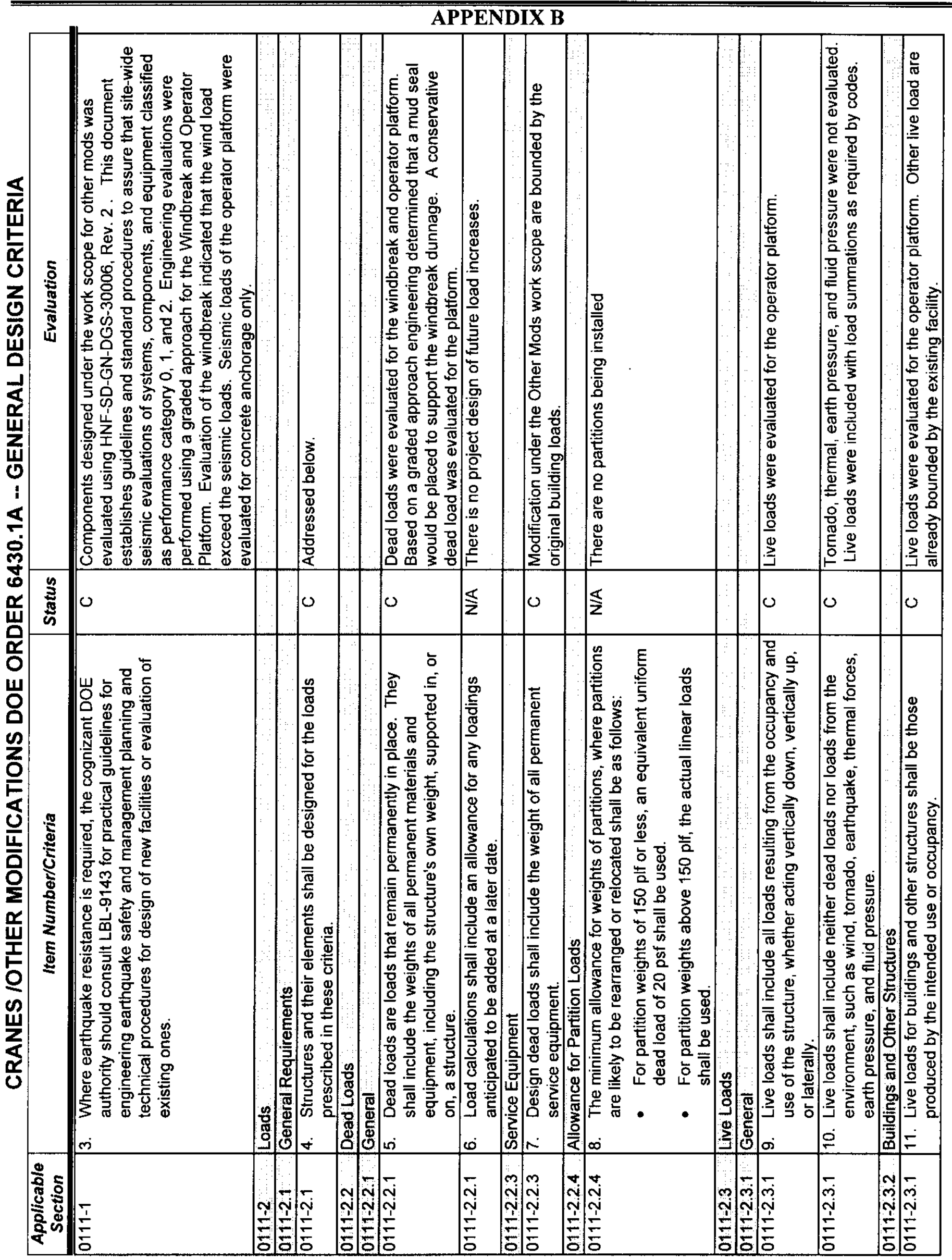




\begin{tabular}{|c|c|c|c|c|c|c|c|c|c|c|c|c|c|c|}
\hline \multicolumn{15}{|c|}{ APPENDIX B } \\
\hline \multirow{3}{*}{\multicolumn{2}{|c|}{ 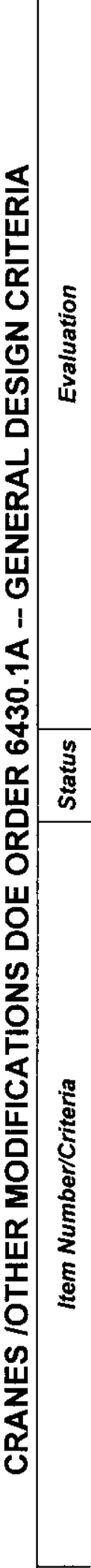 }} & 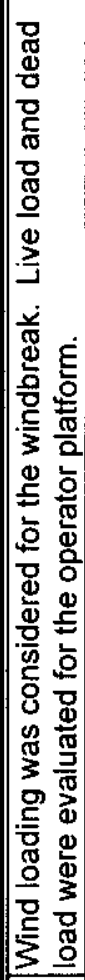 & 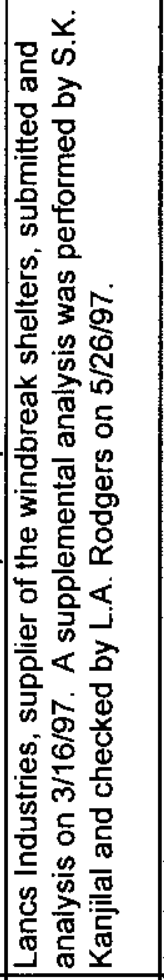 & & 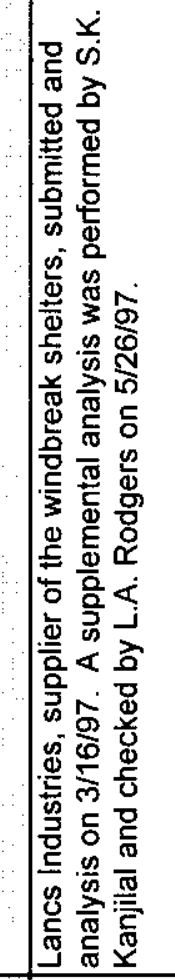 & & 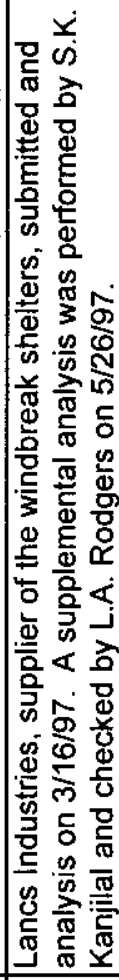 & 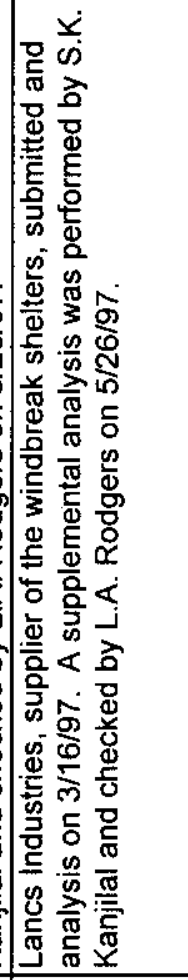 & 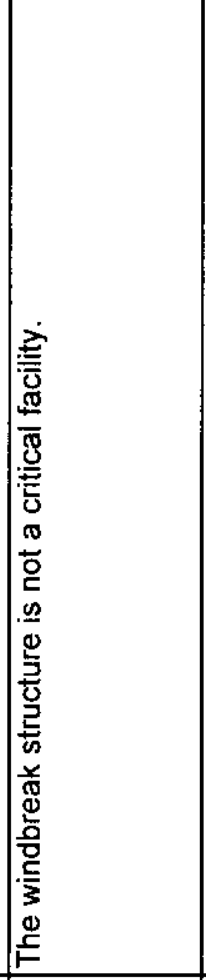 & 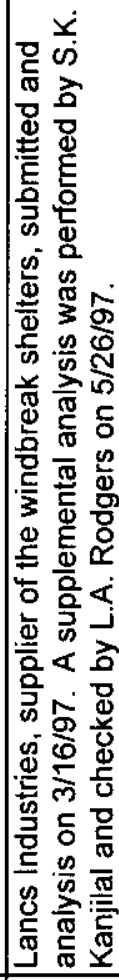 & 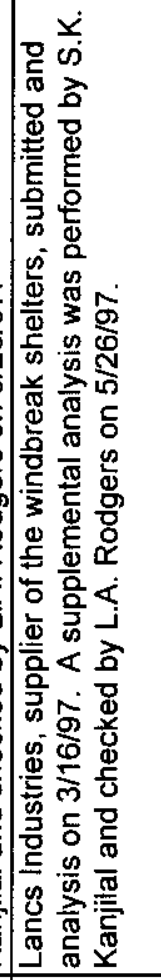 & & 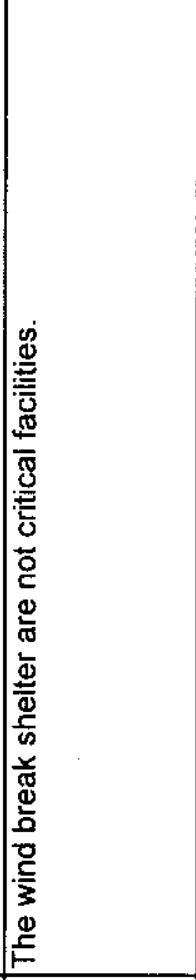 & 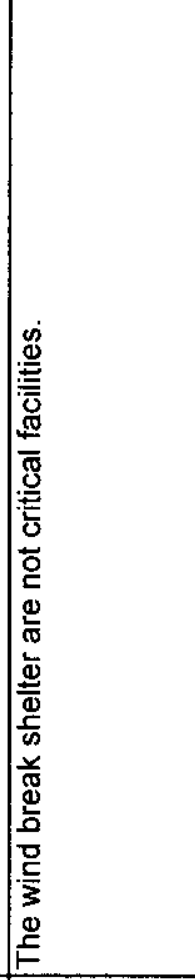 \\
\hline & & 0 & 0 & & 0 & & 0 & 0 & 0 & 0 & 0 & & $\frac{\$}{z}$ & $\frac{1}{z}$ \\
\hline & & 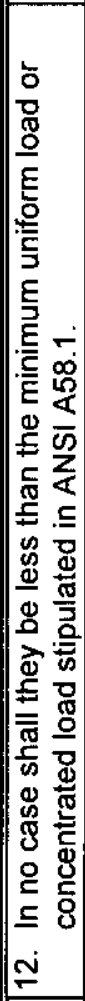 & 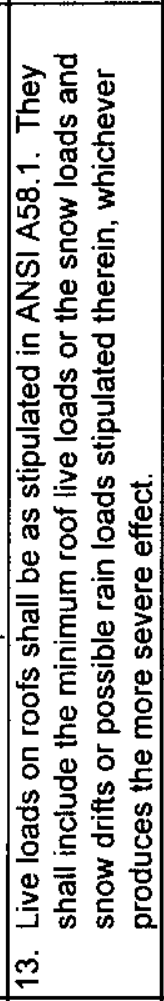 & 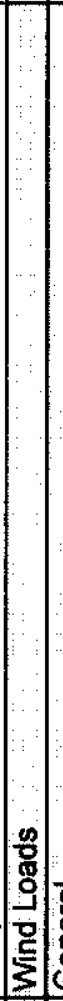 & 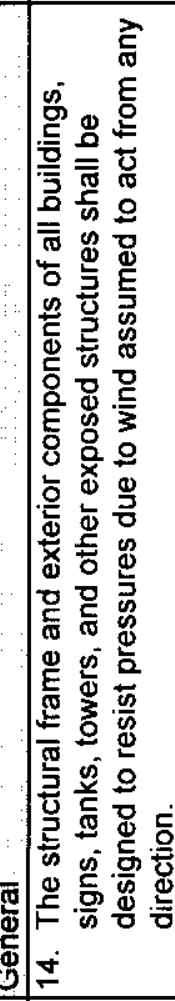 & & 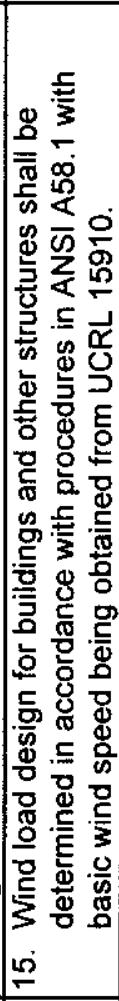 & 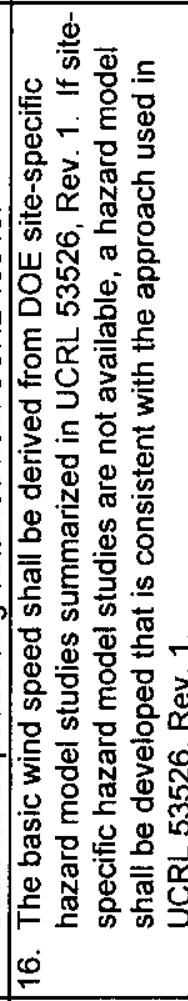 & 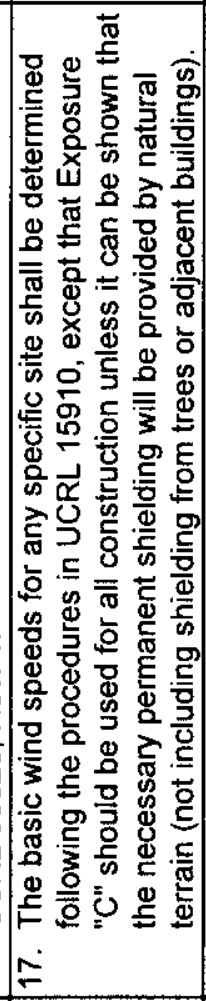 & 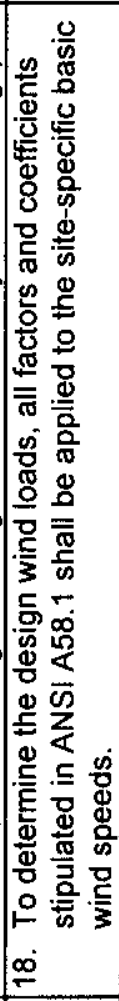 & 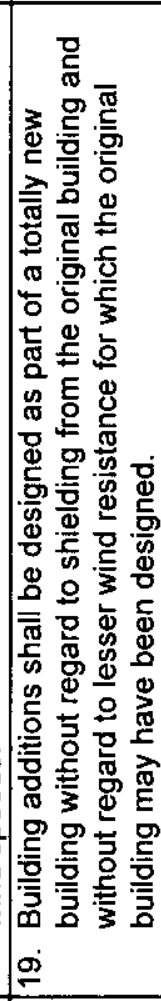 & 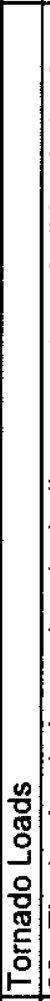 & 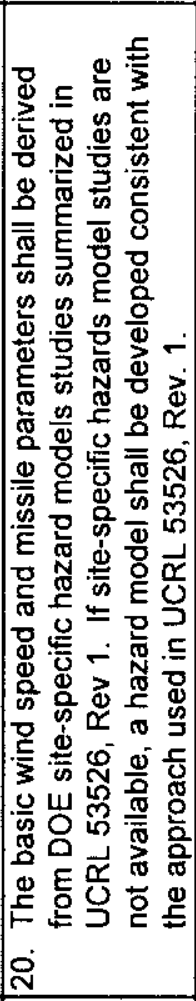 & 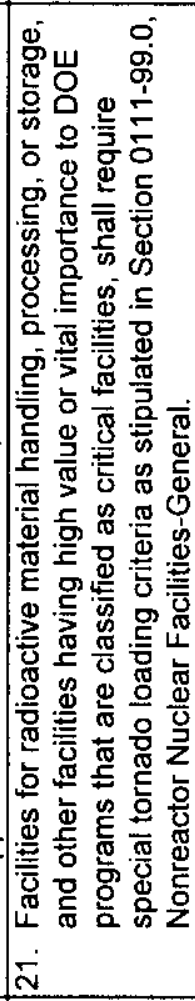 \\
\hline & 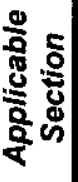 & 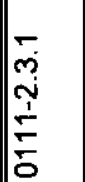 & 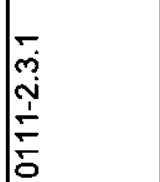 & & 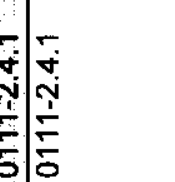 & & & $\stackrel{\grave{Y}}{\check{Y}}$ & $\stackrel{\dddot{q}}{\mathscr{q}}$ & 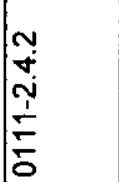 & 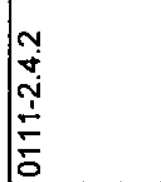 & & & $\frac{\stackrel{2}{\sim}}{\stackrel{\sim}{\sigma}}$ \\
\hline
\end{tabular}


SNF-4775, Rev. 2

Project A.5/A.6

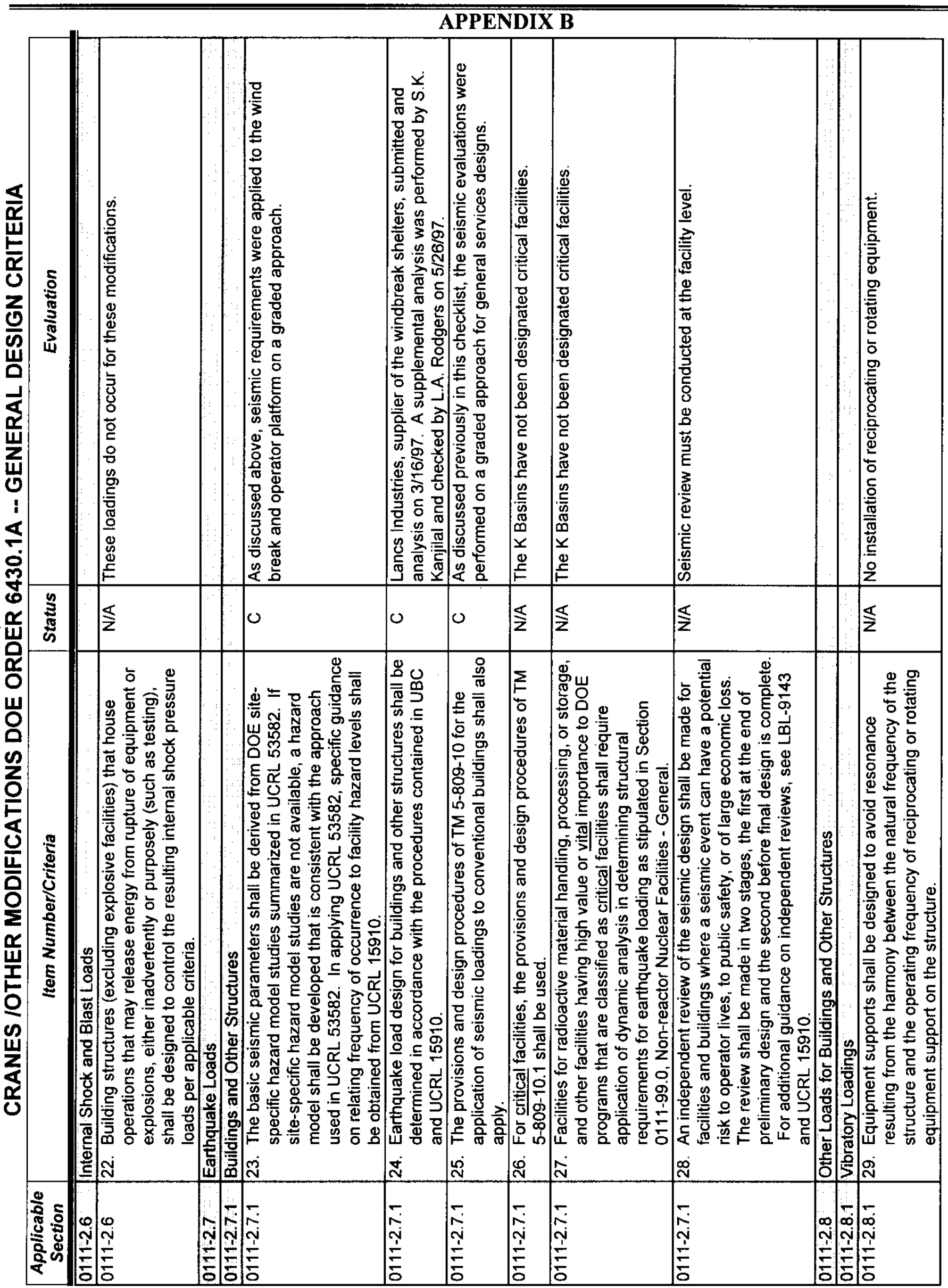

Page B-32 
SNF-4775, Rev. 2

Project A.5/A.6

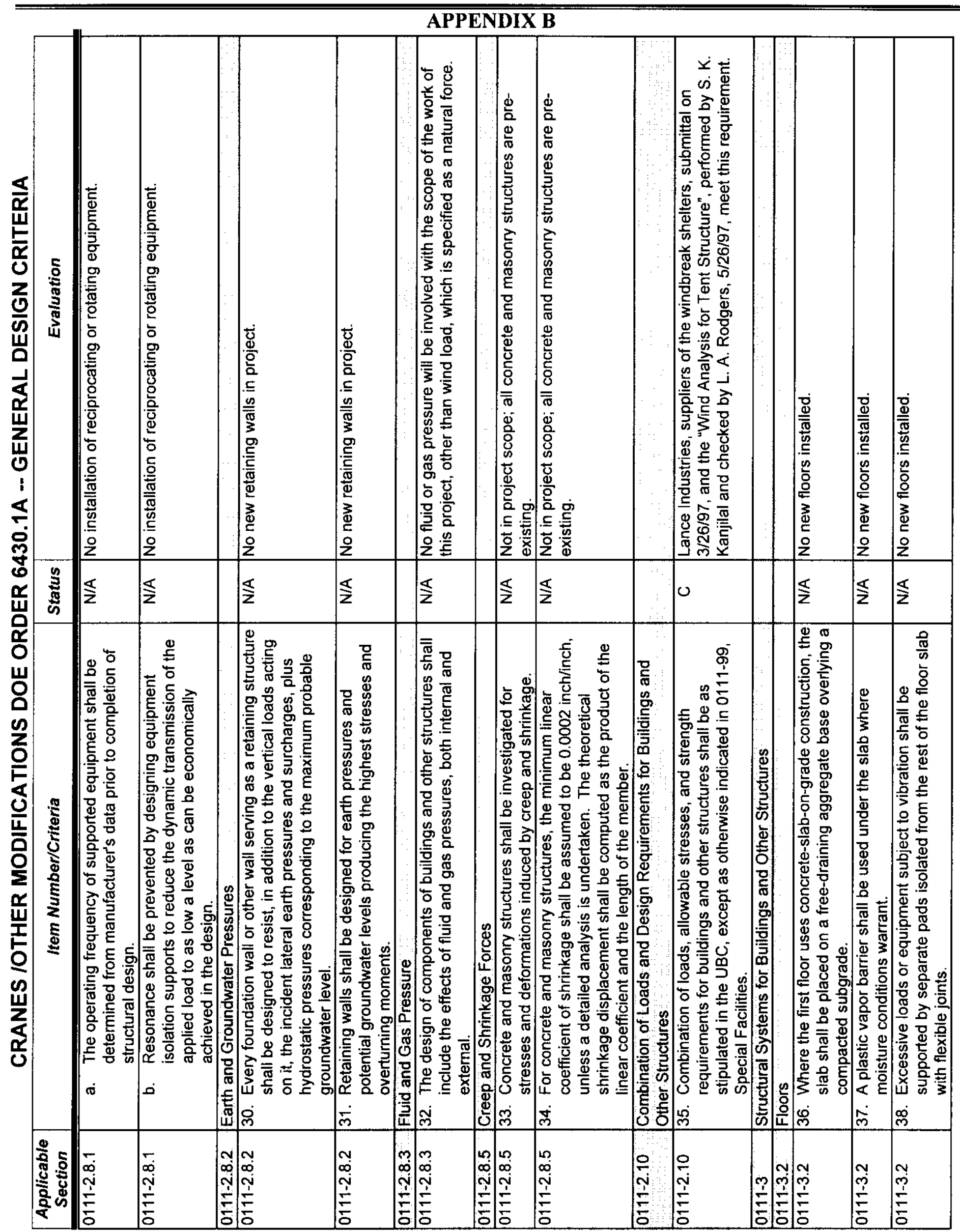

Page B-33 


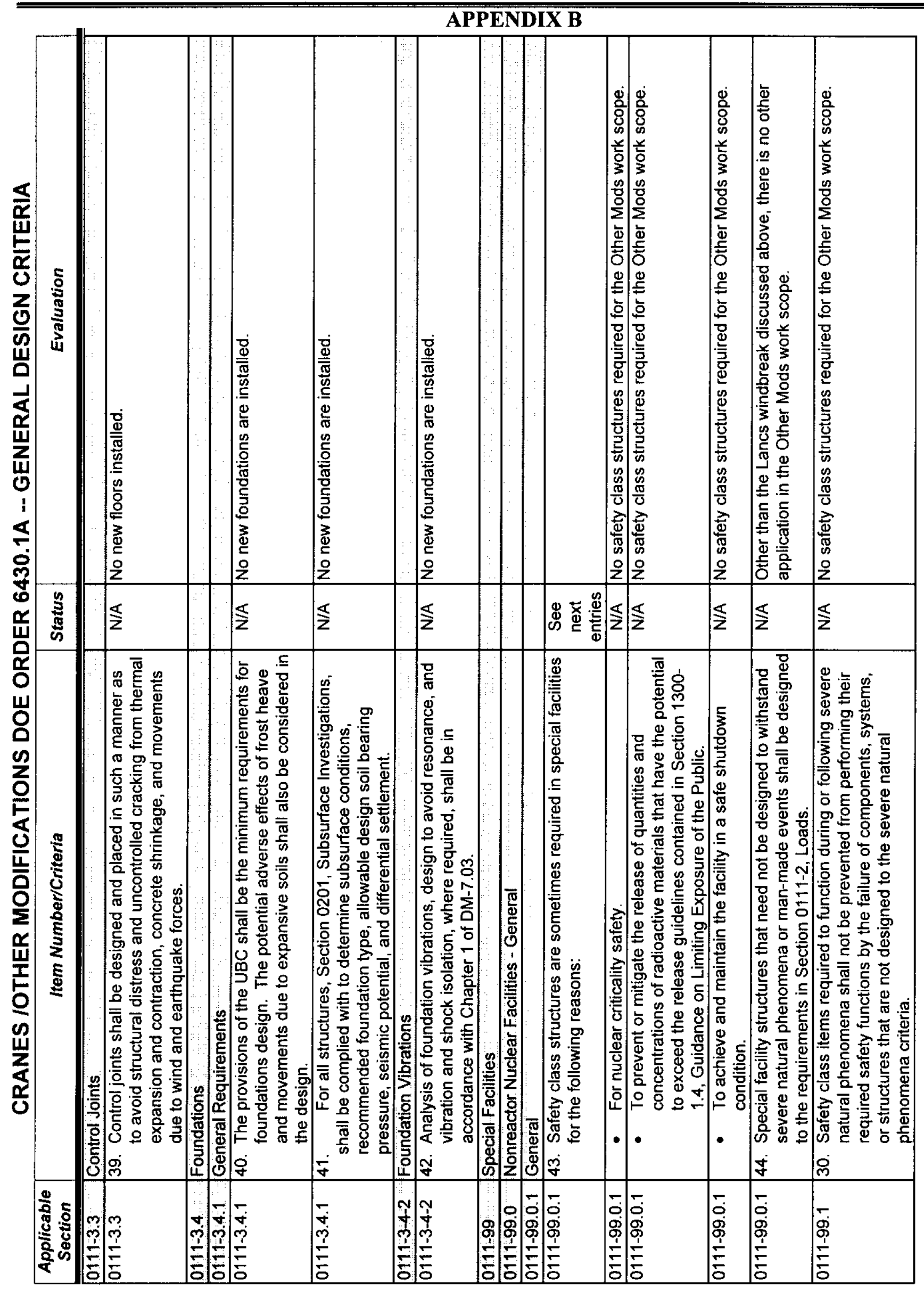




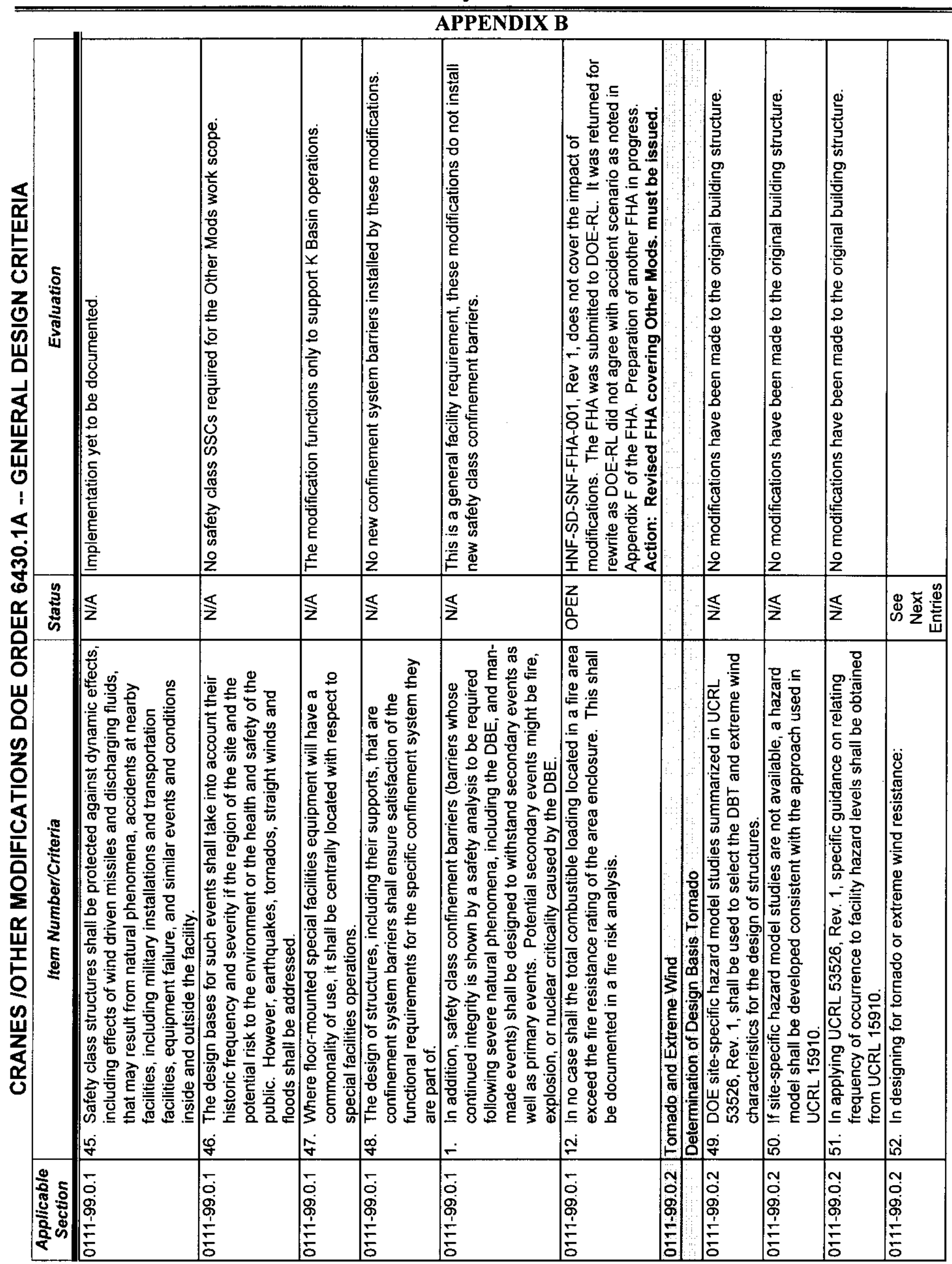




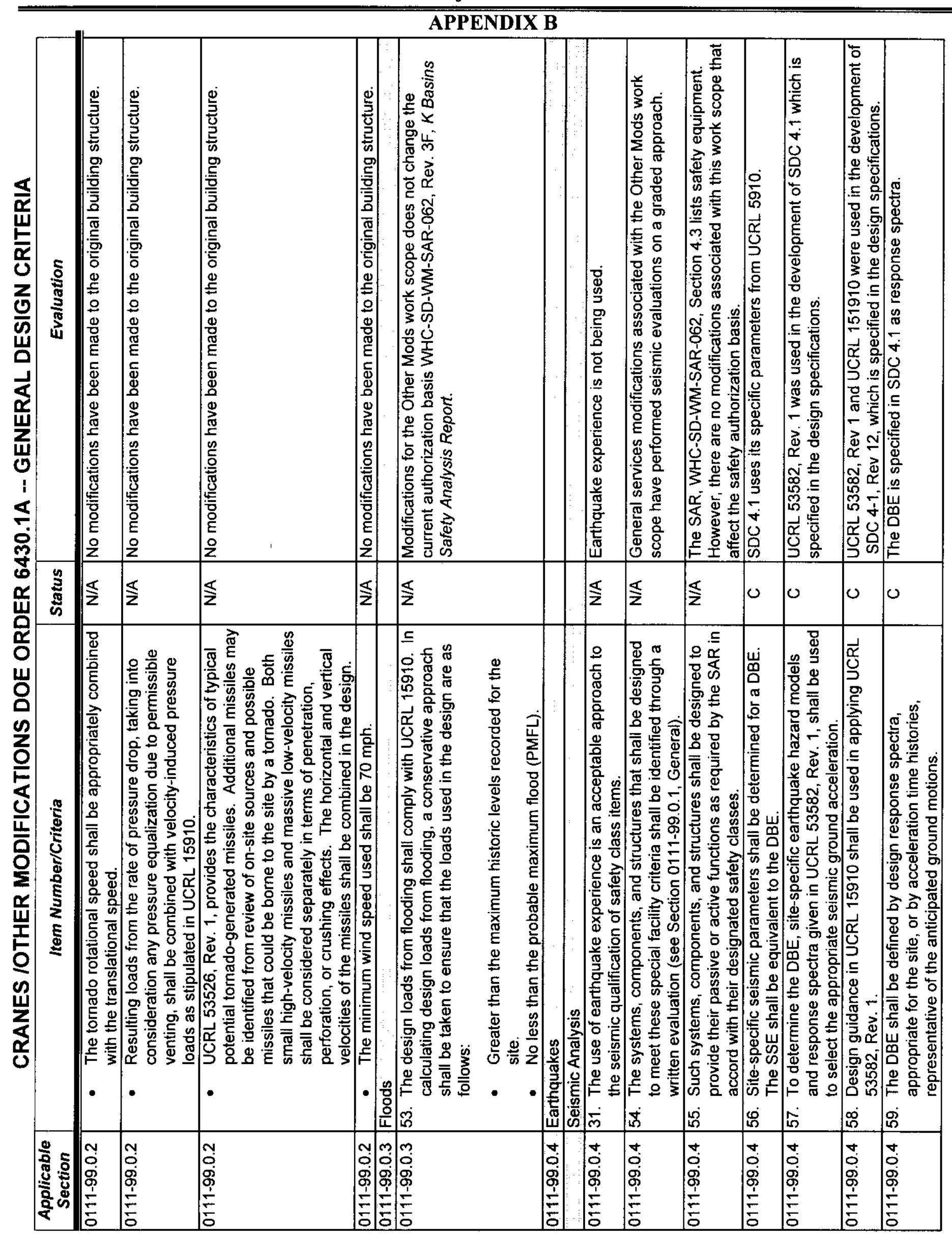




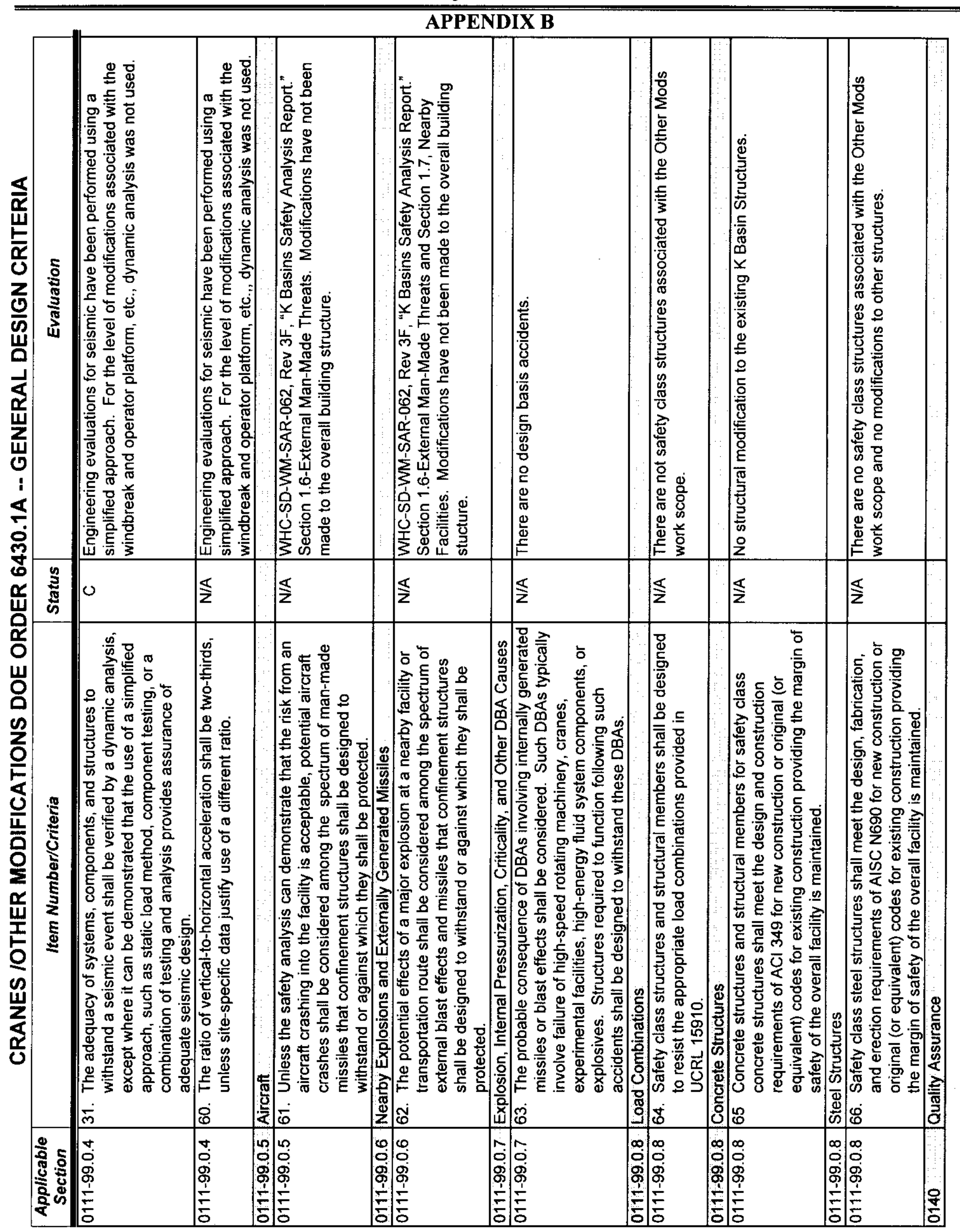




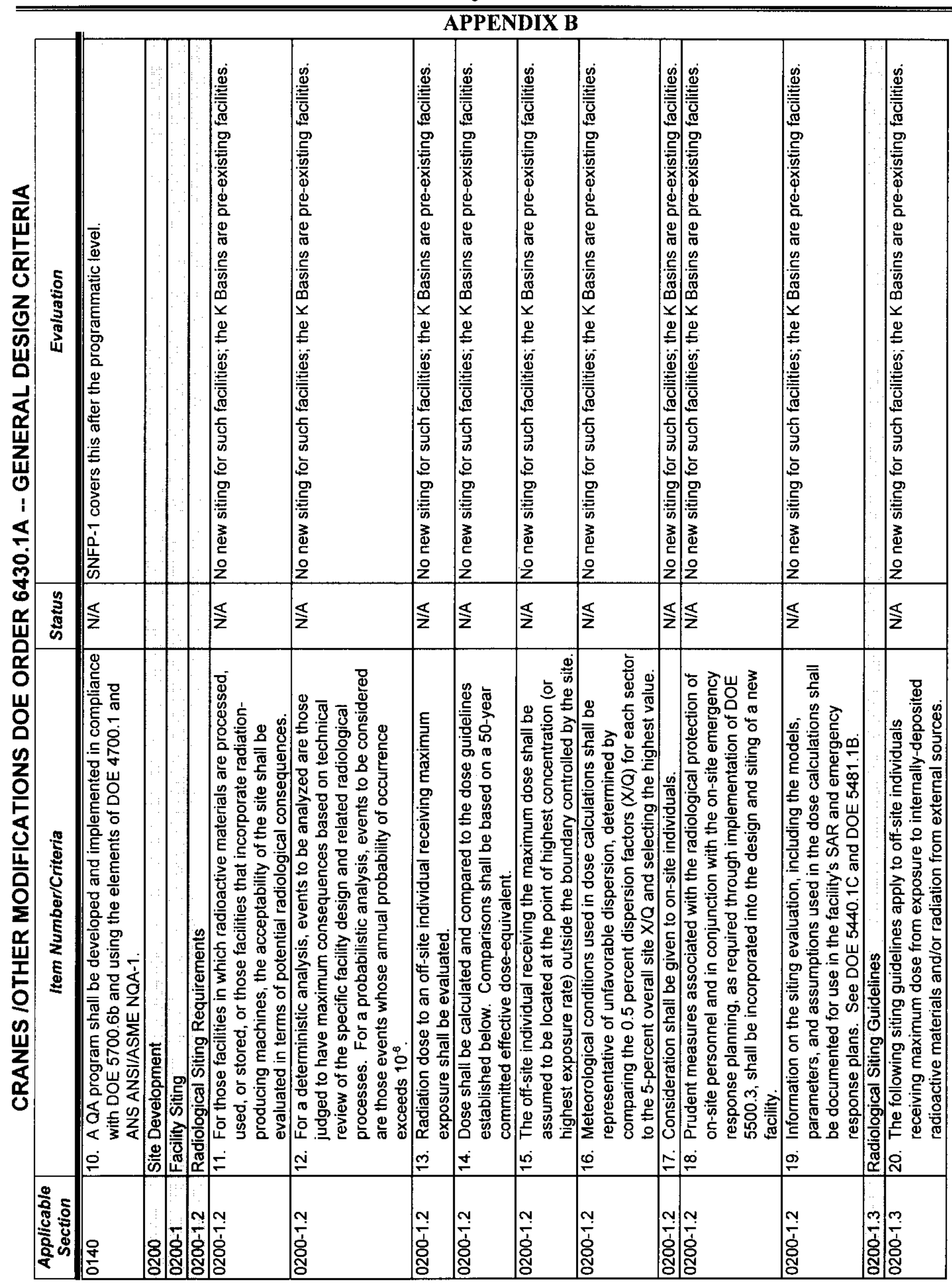




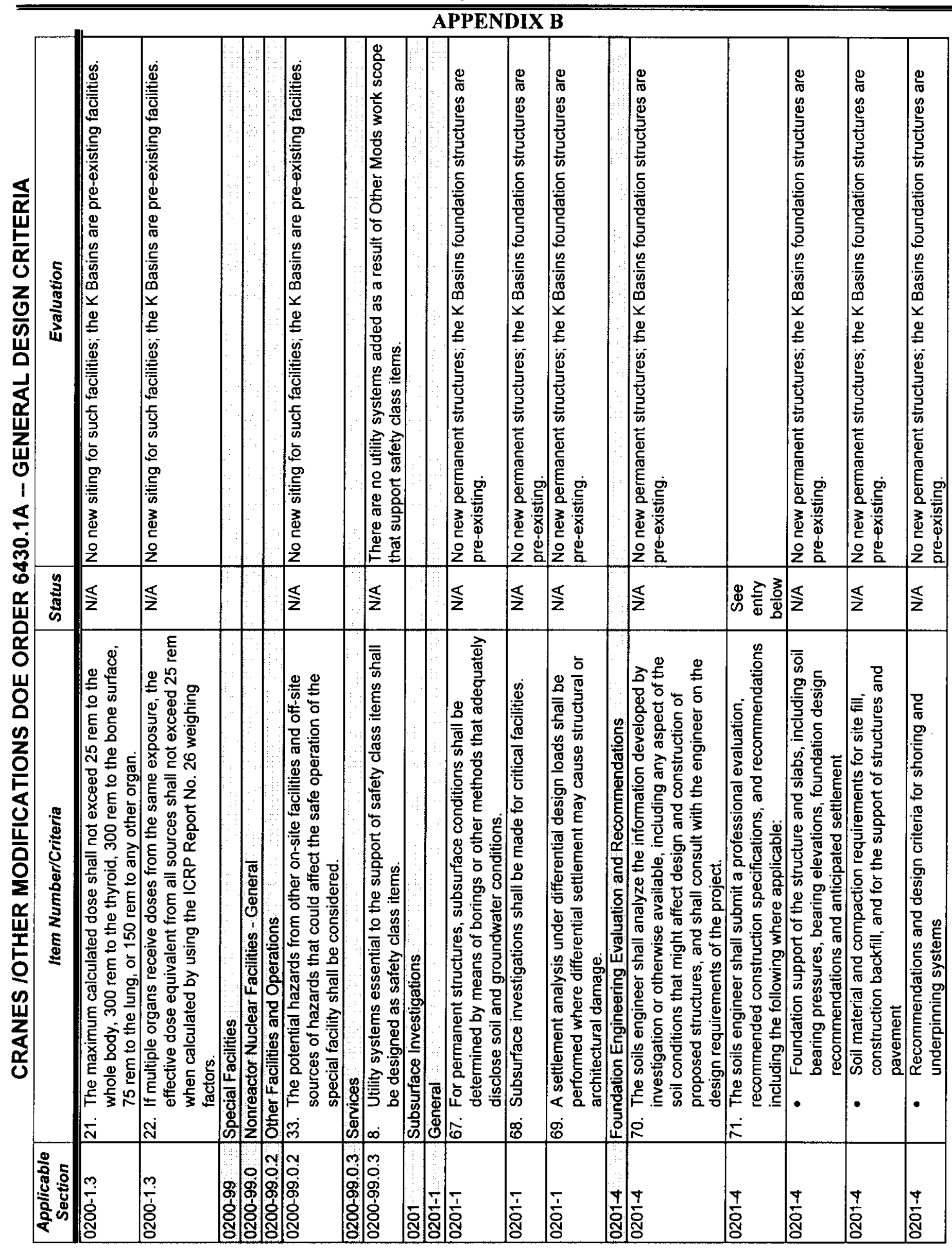




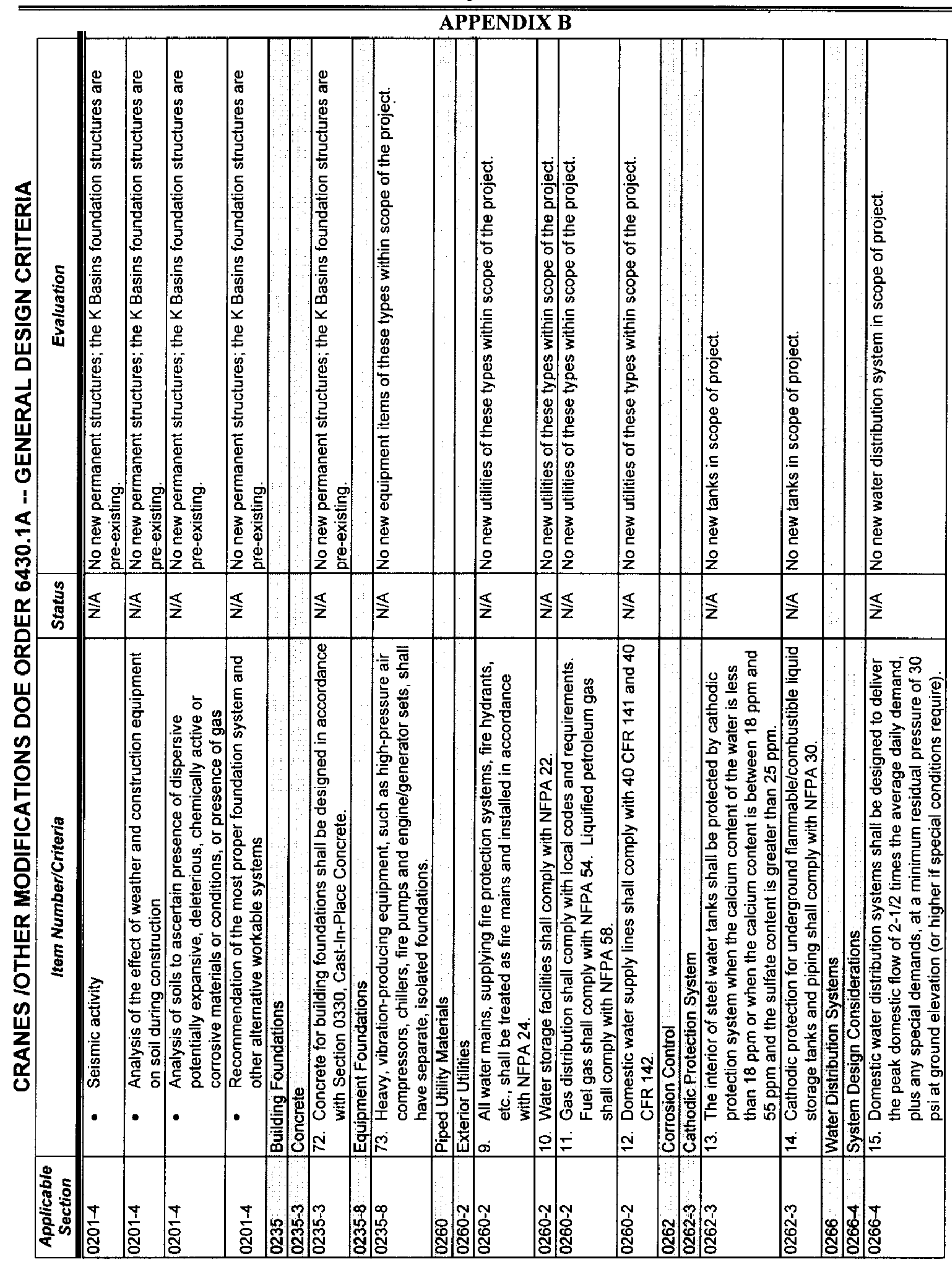




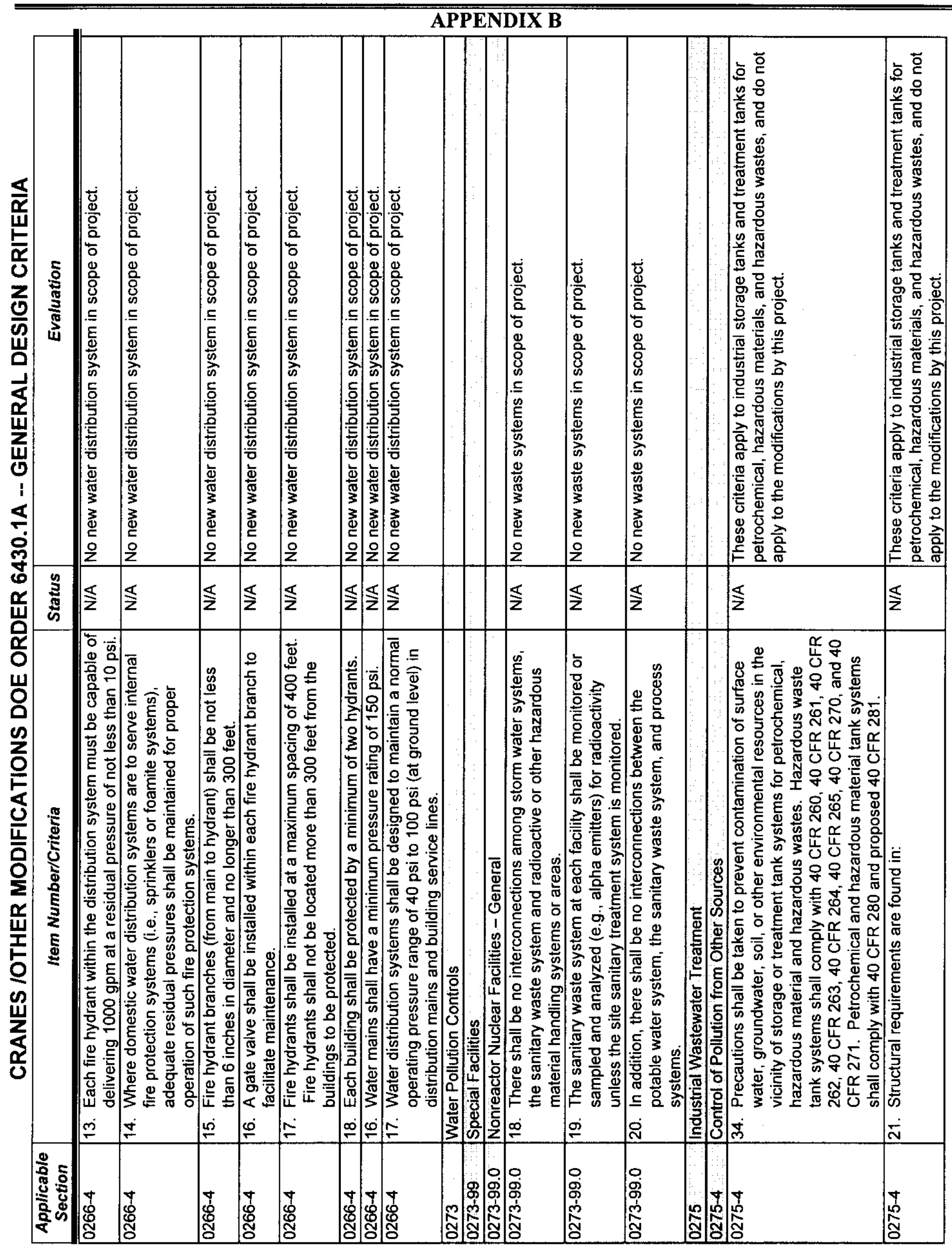


SNF-4775, Rev. 2

Project A.5/A.6

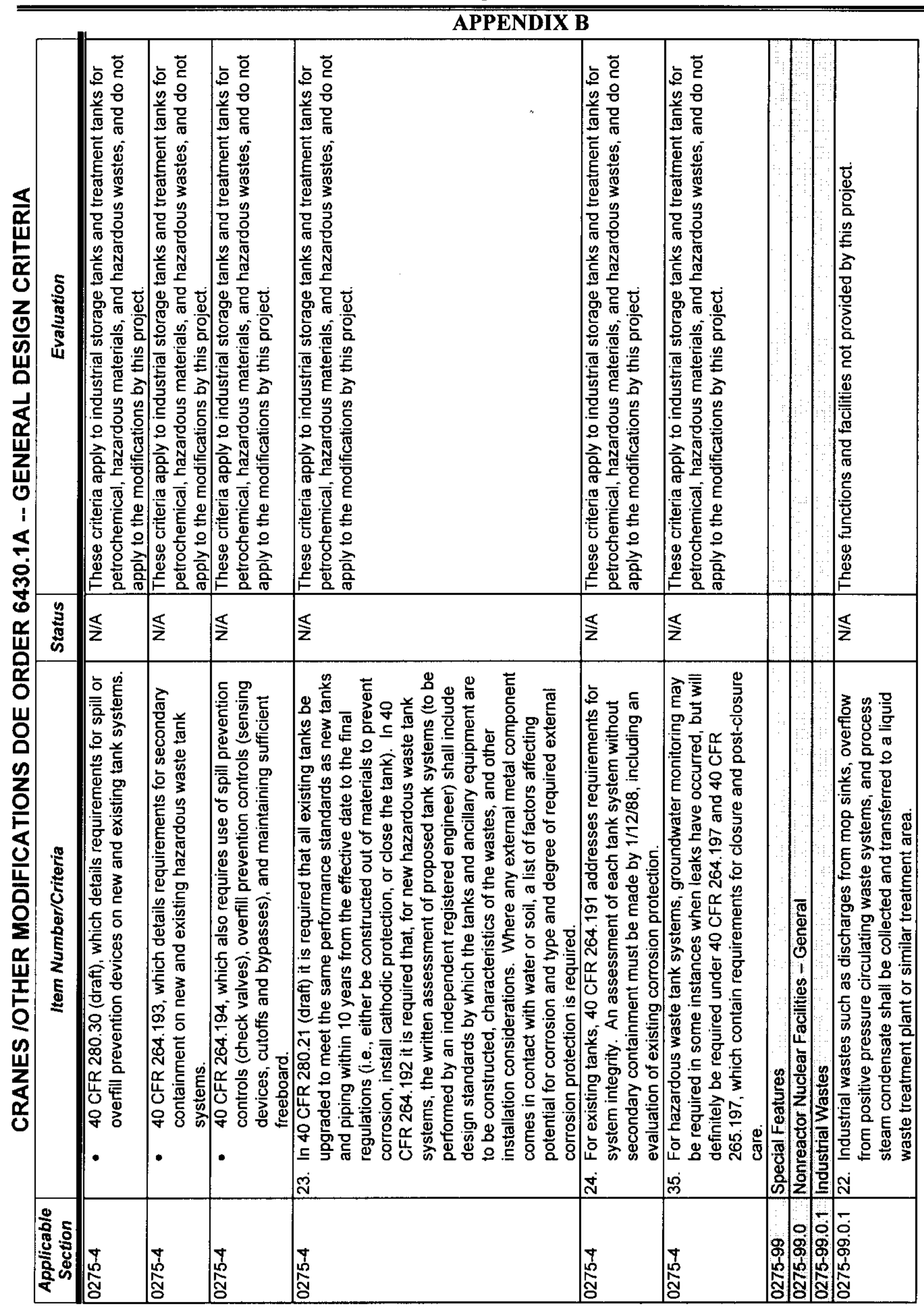

Page B-42 
SNF-4775, Rev. 2

Project A.5/A.6

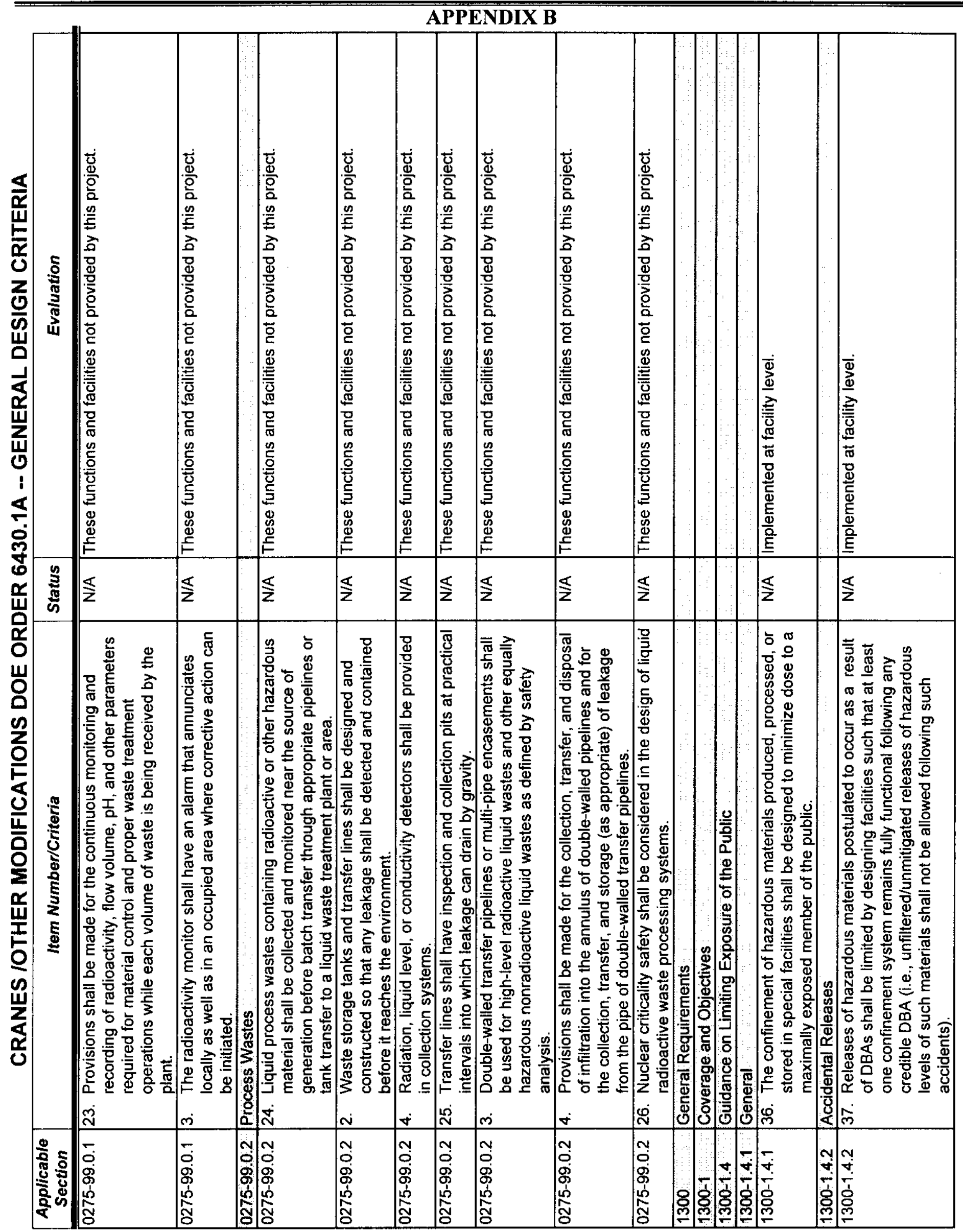




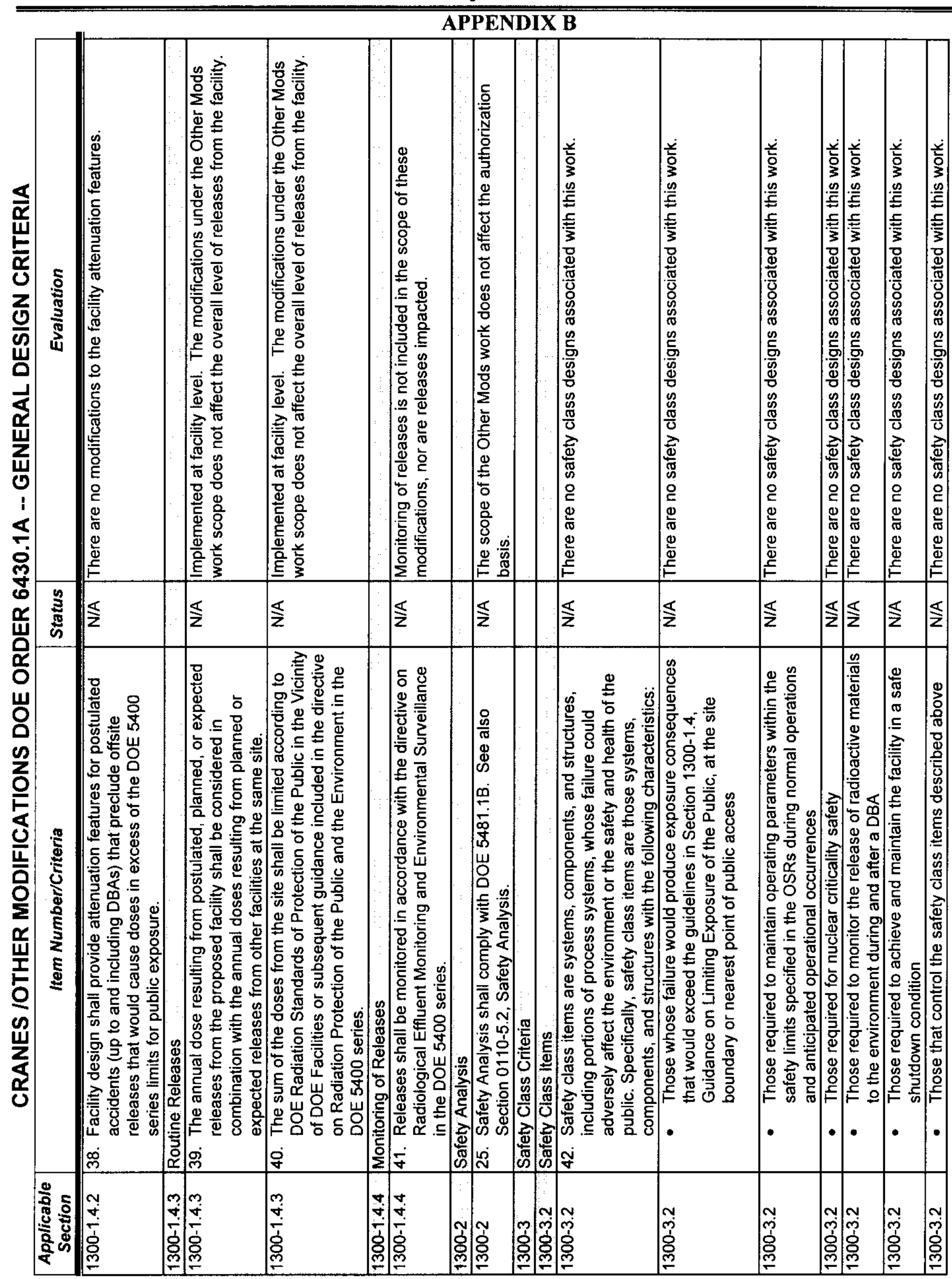




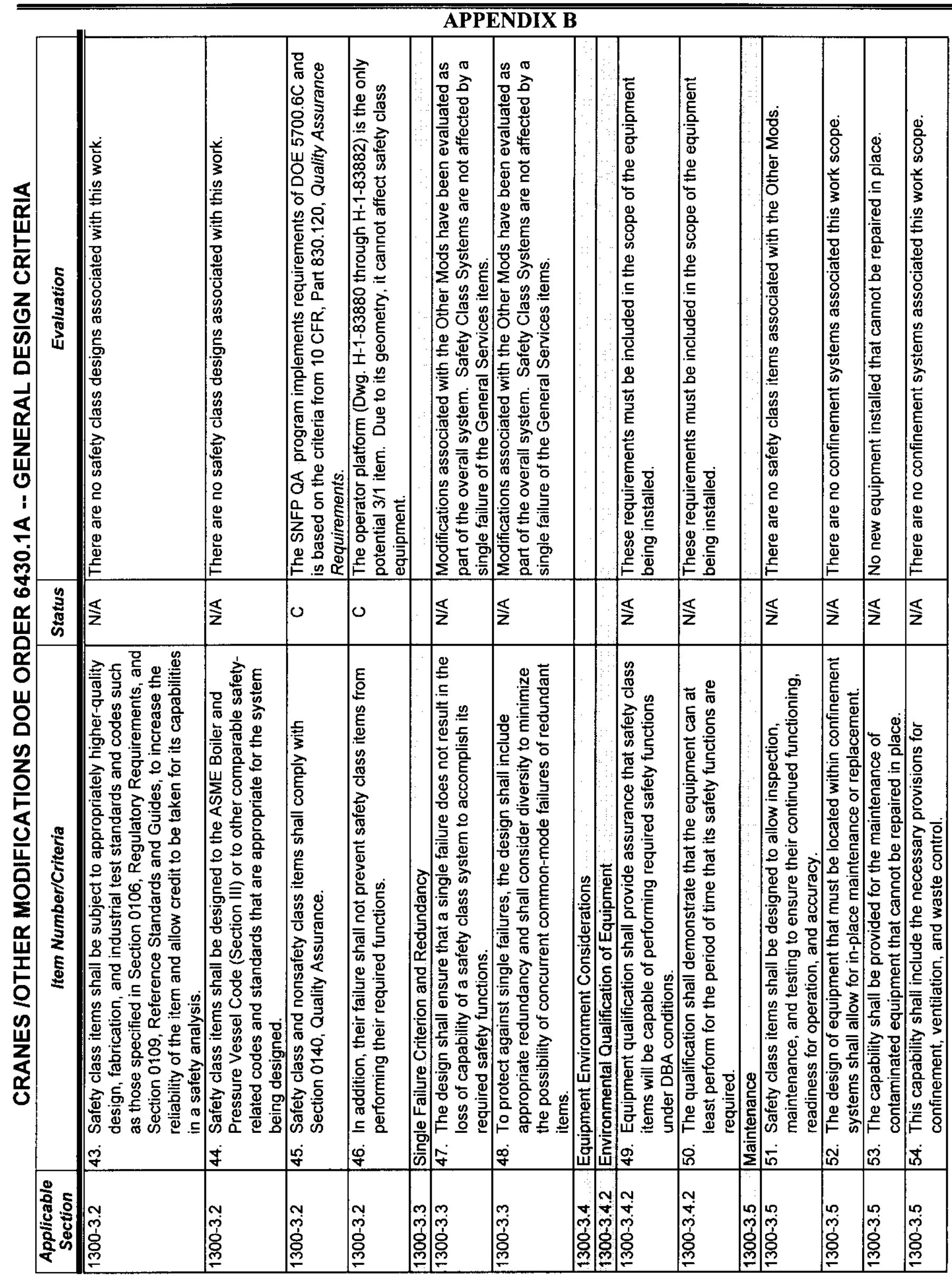


SNF-4775, Rev. 2

Project A.5/A.6

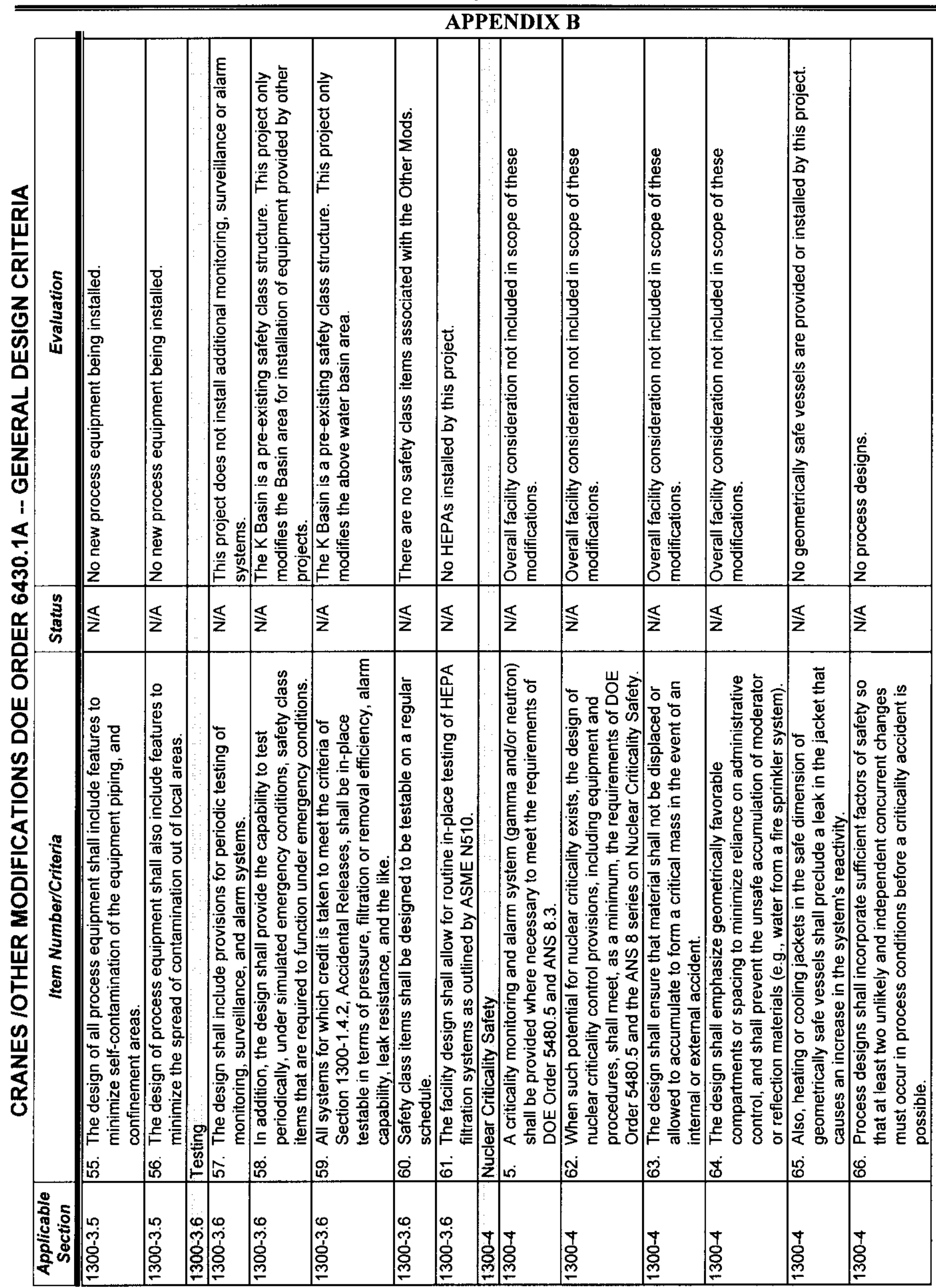


SNF-4775, Rev. 2

Project A.5/A.6

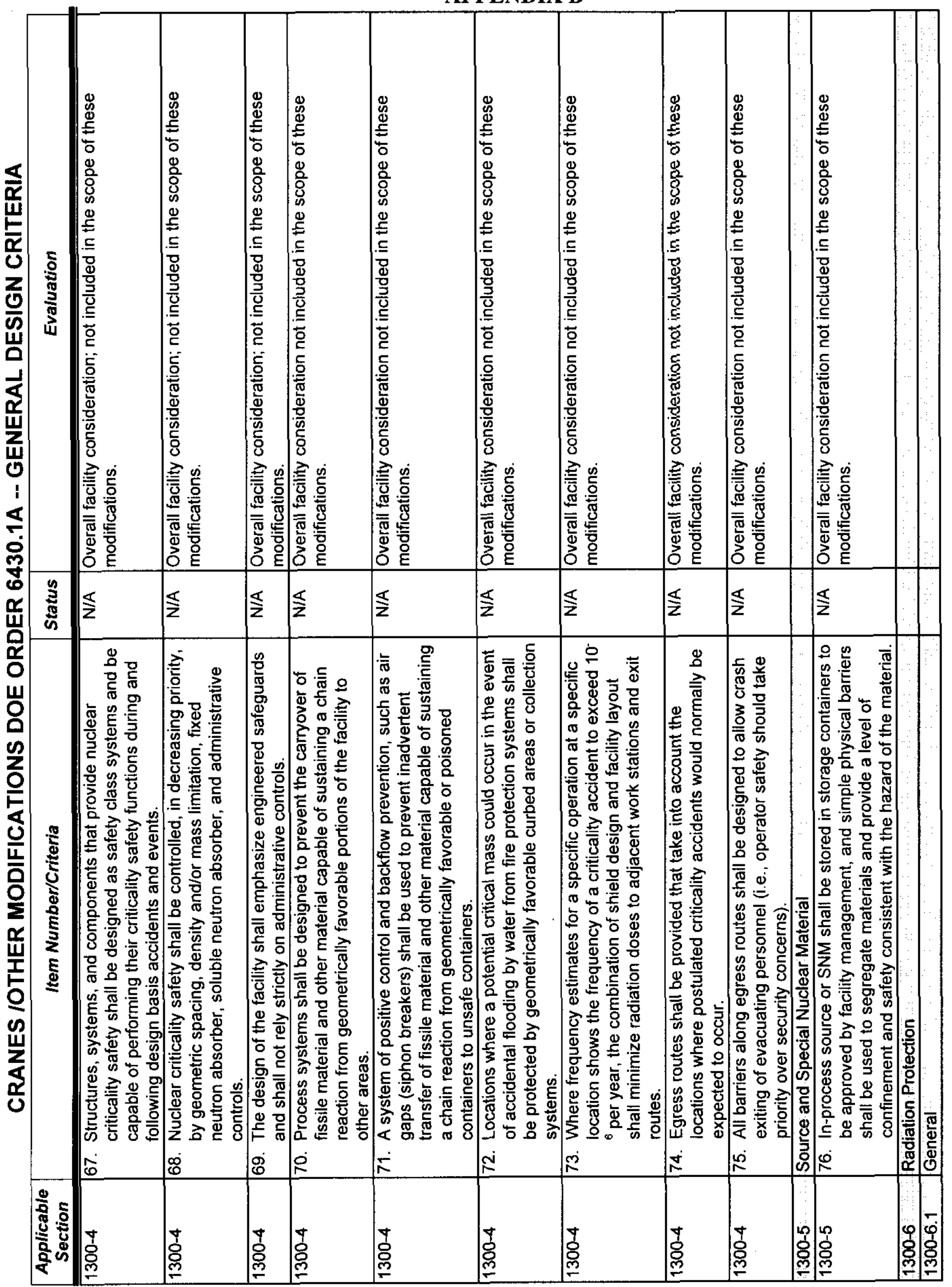




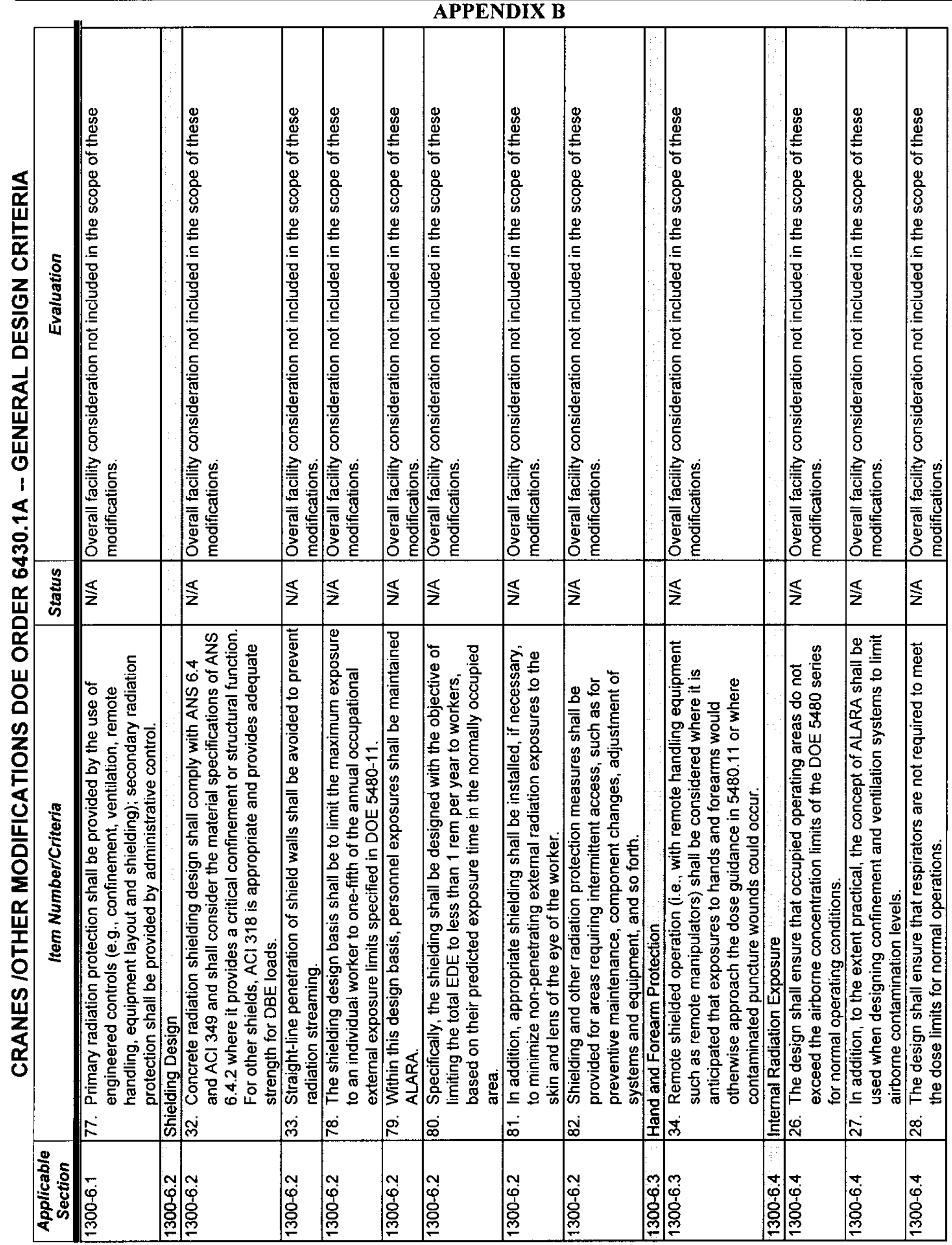


SNF-4775, Rev. 2

Project A.5/A.6

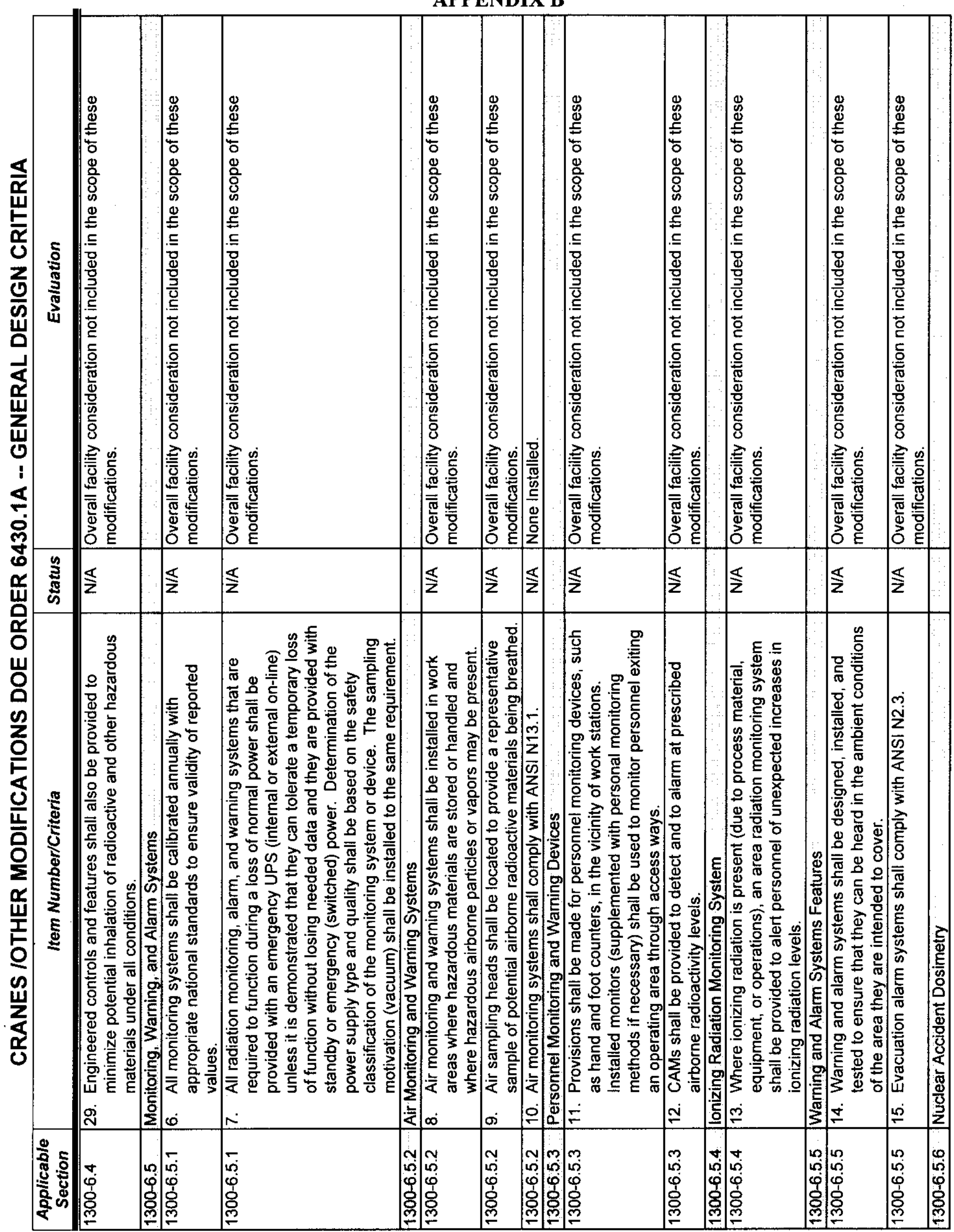




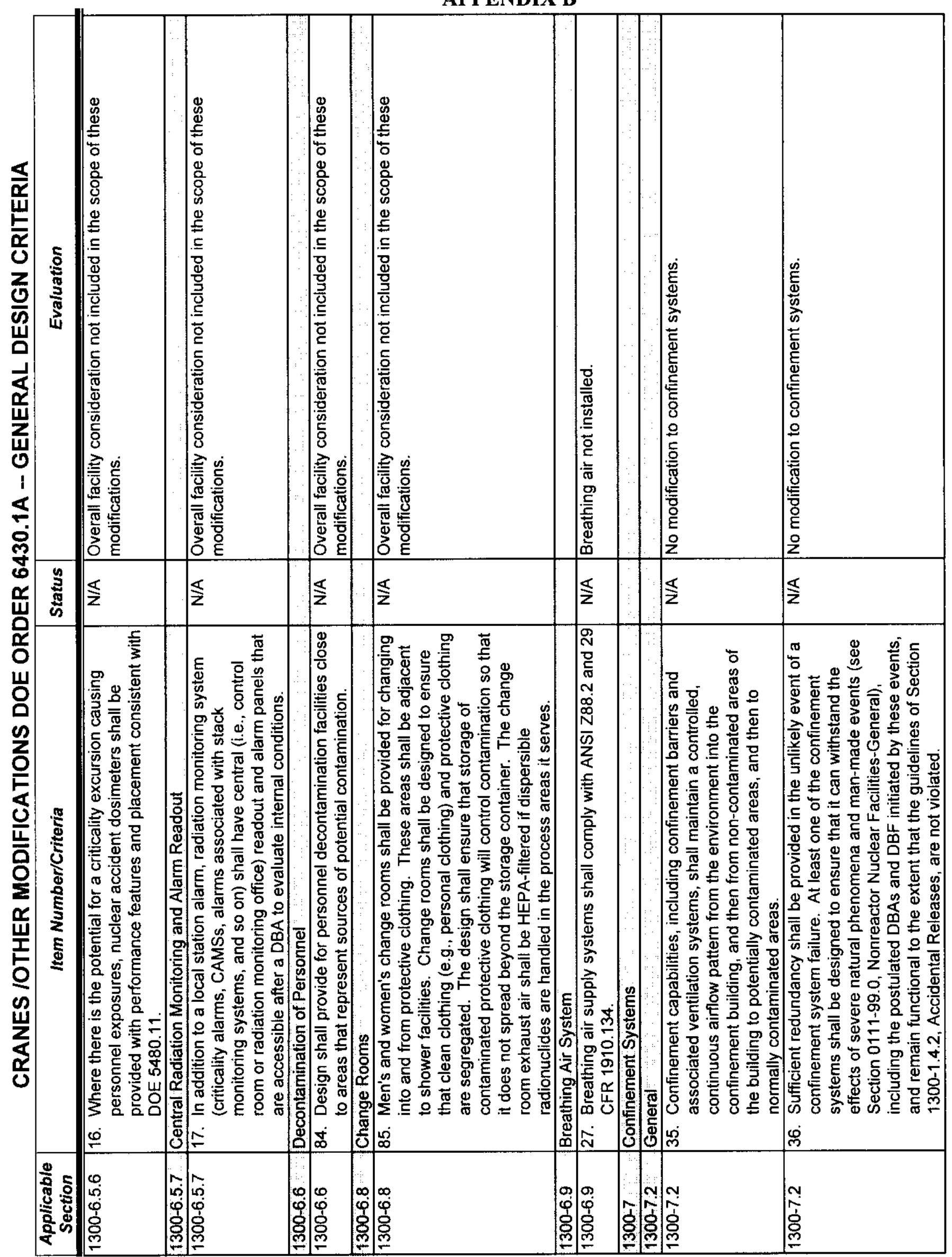




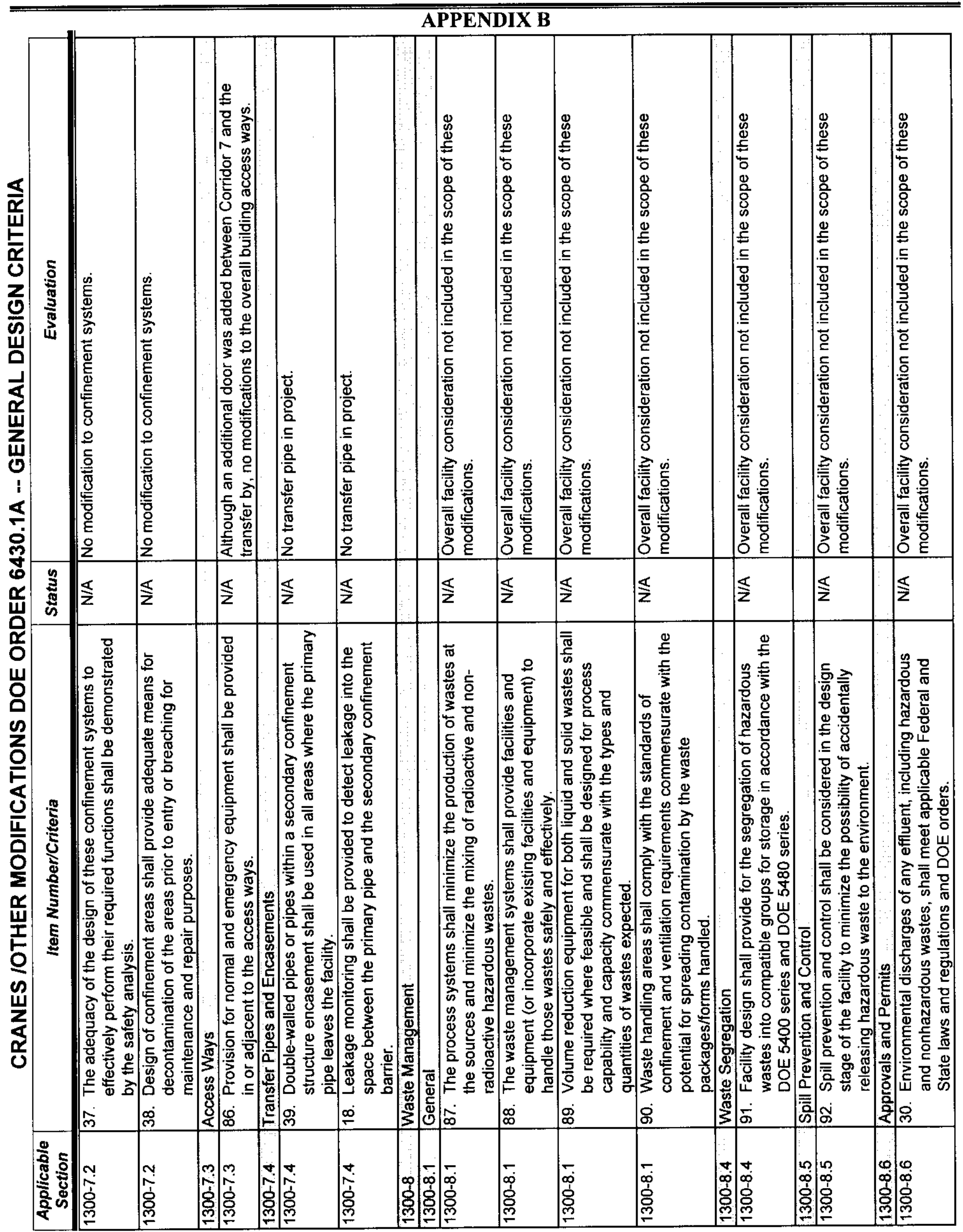




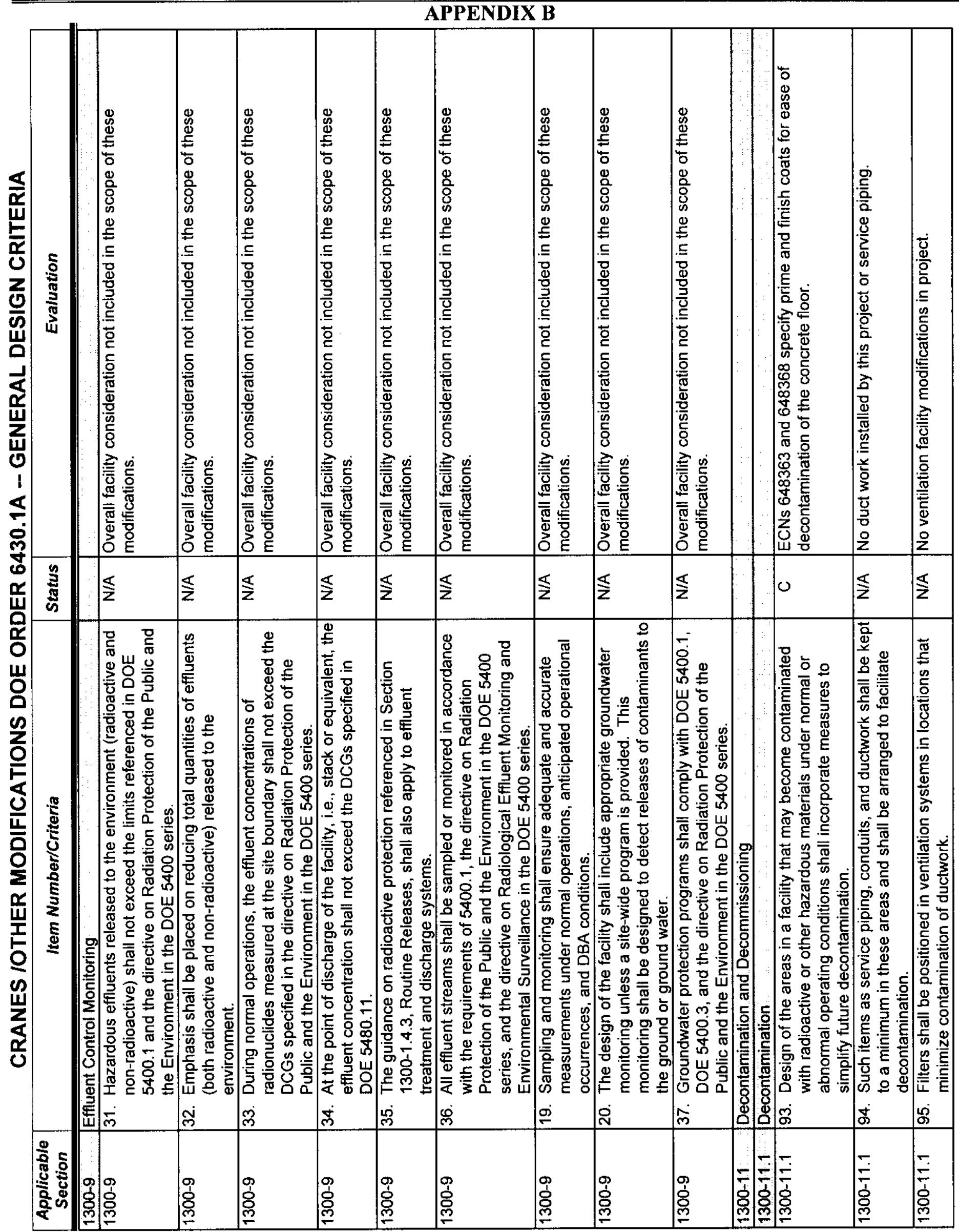


SNF-4775, Rev. 2

Project A.5/A.6

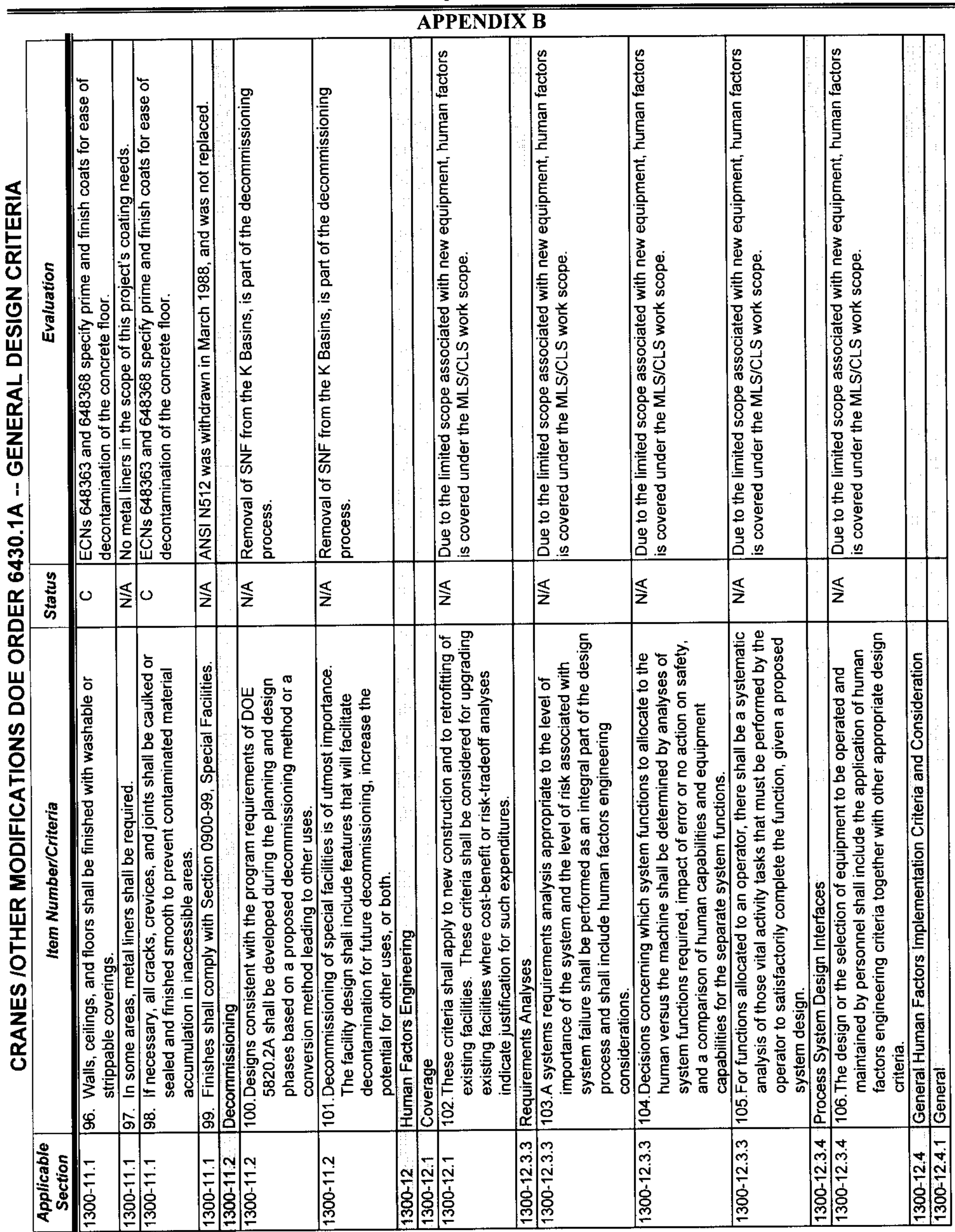


SNF-4775, Rev. 2

Project A.5/A.6

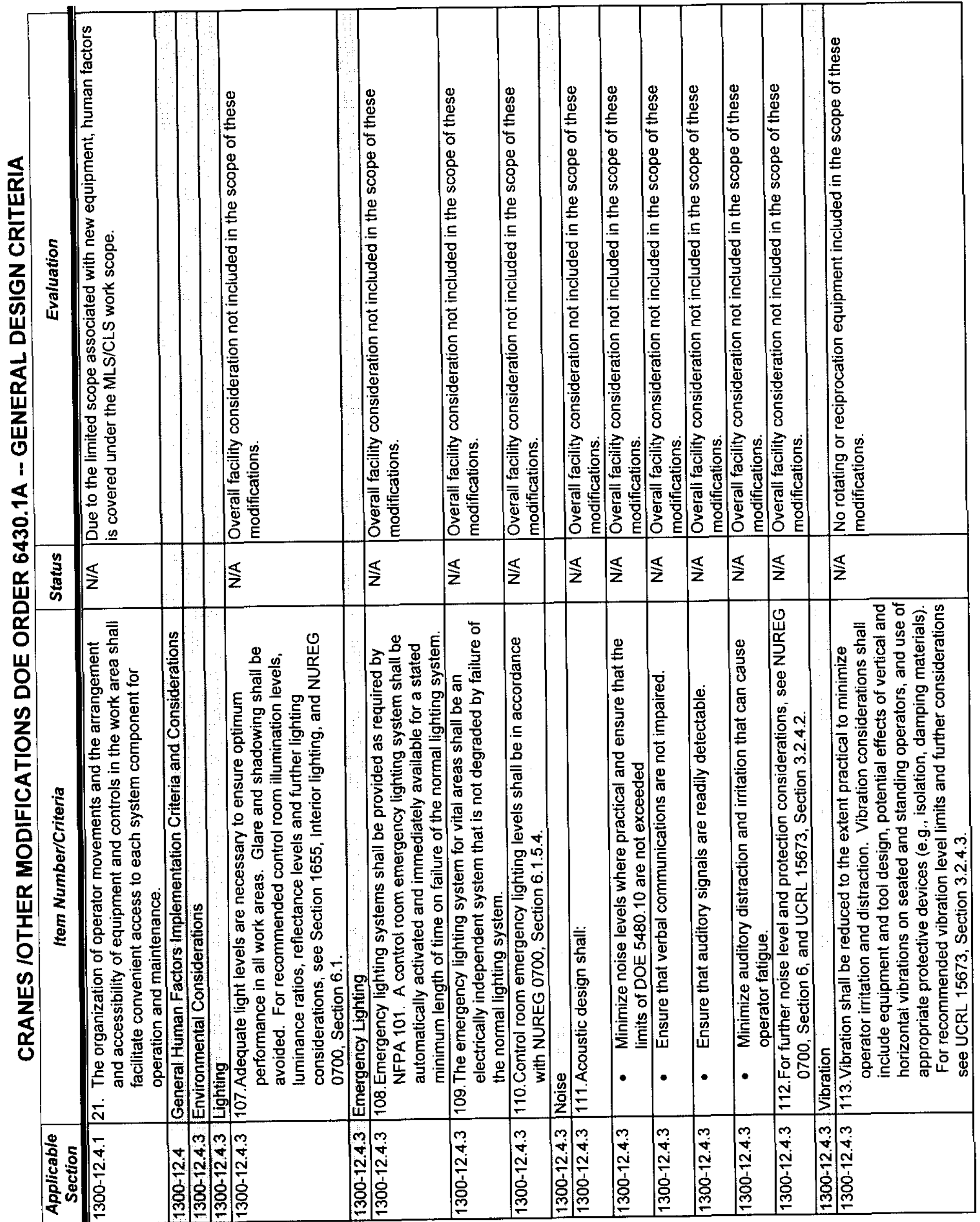




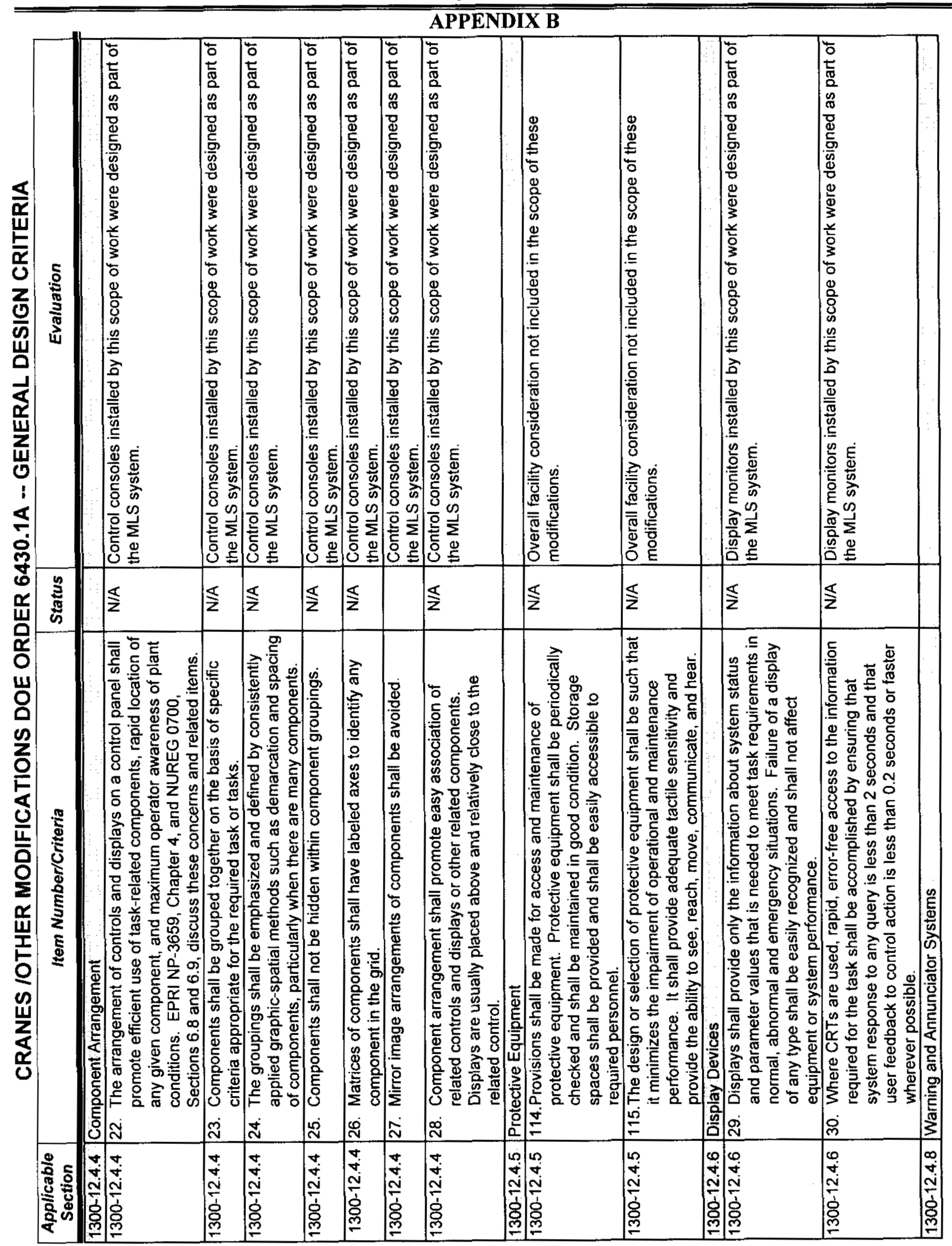




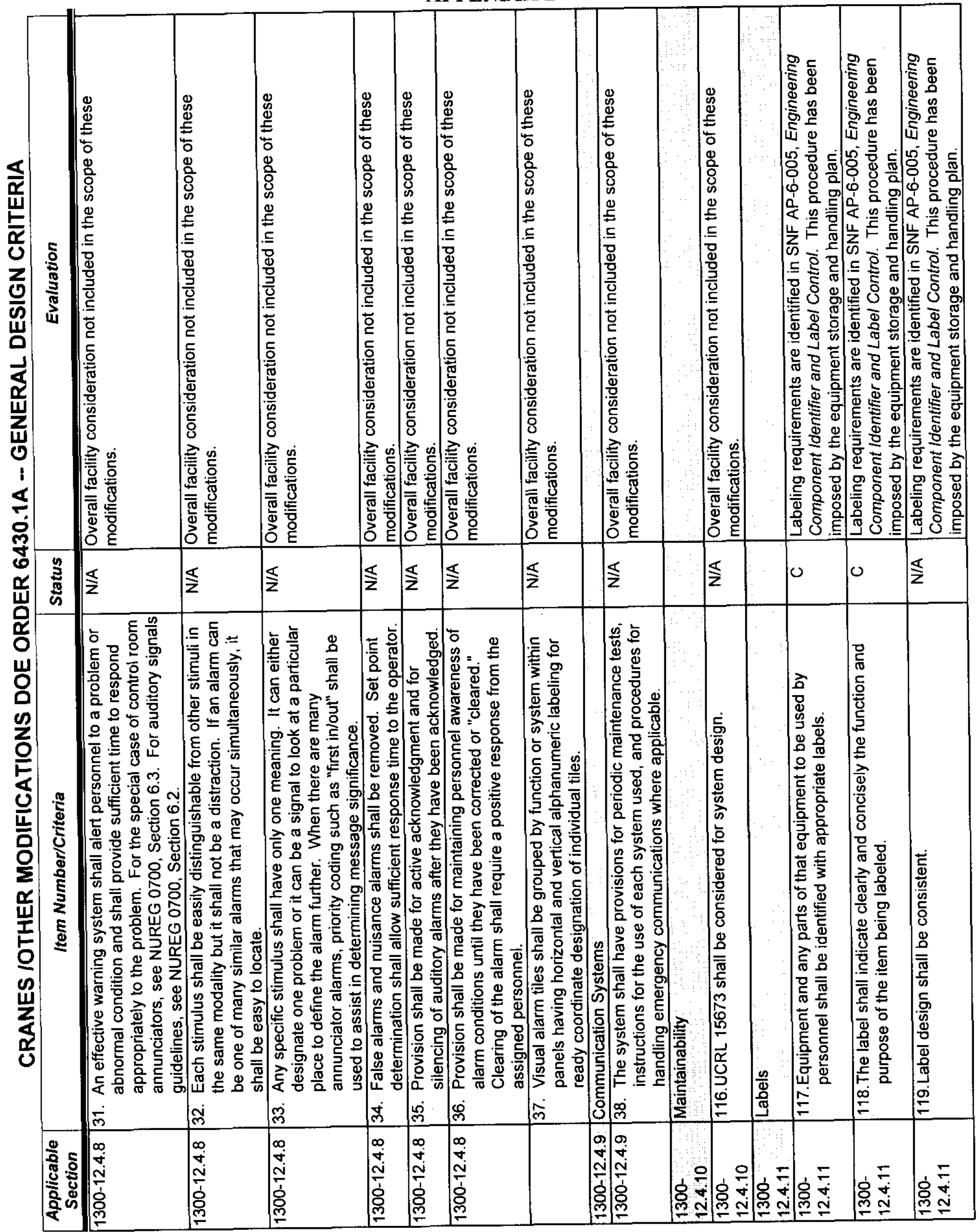


SNF-4775, Rev. 2

Project A.5/A.6

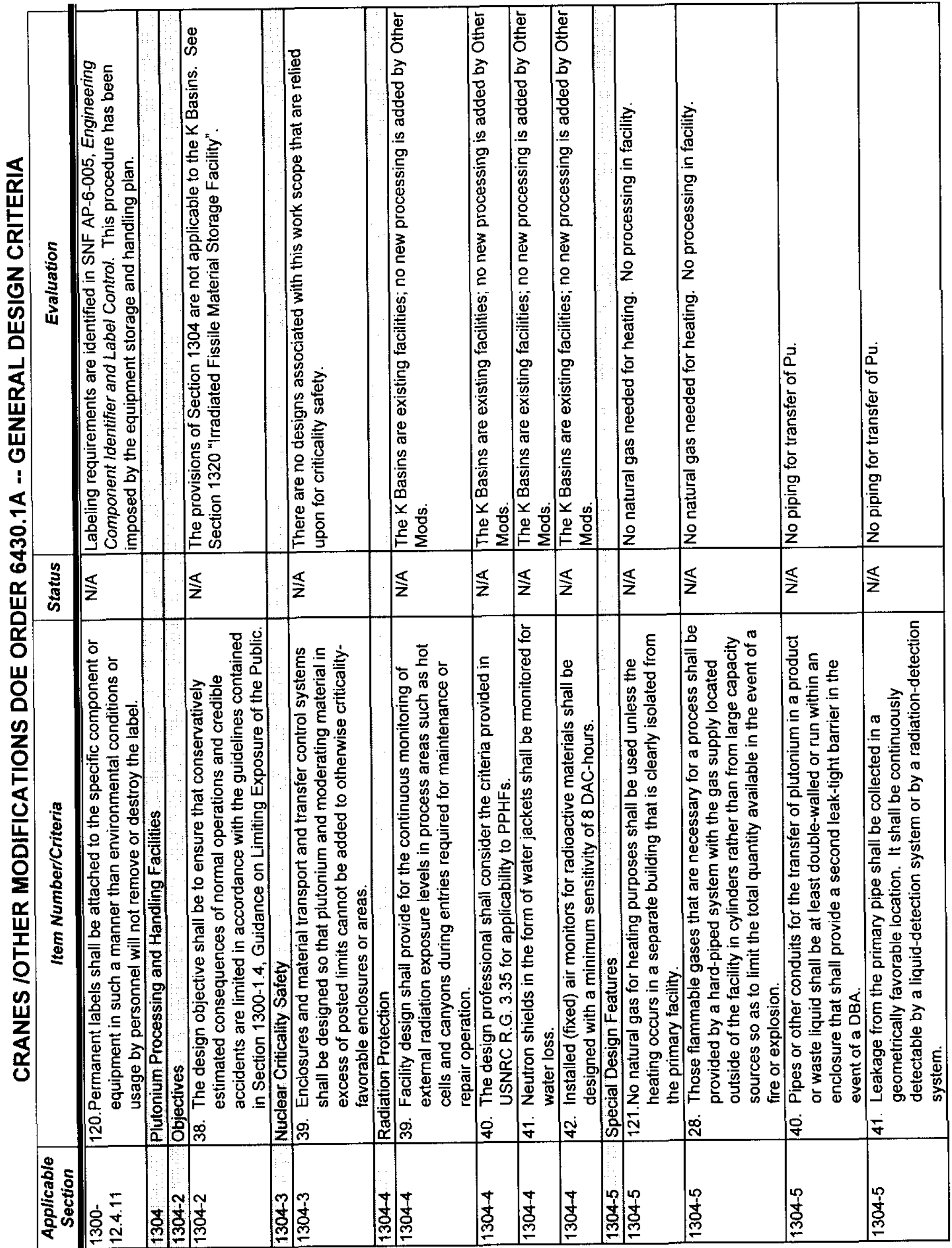


SNF-4775, Rev. 2

Project A.5/A.6

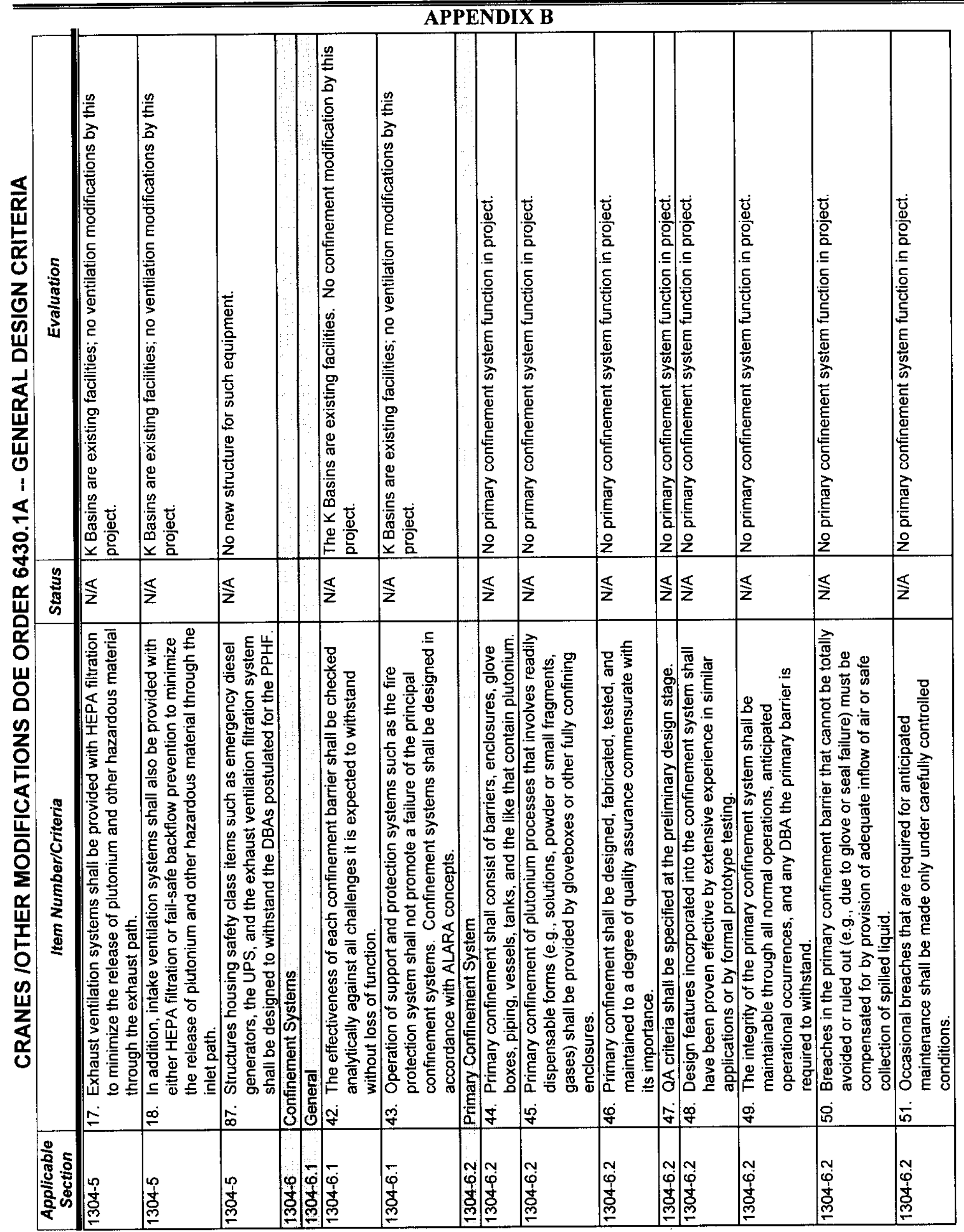




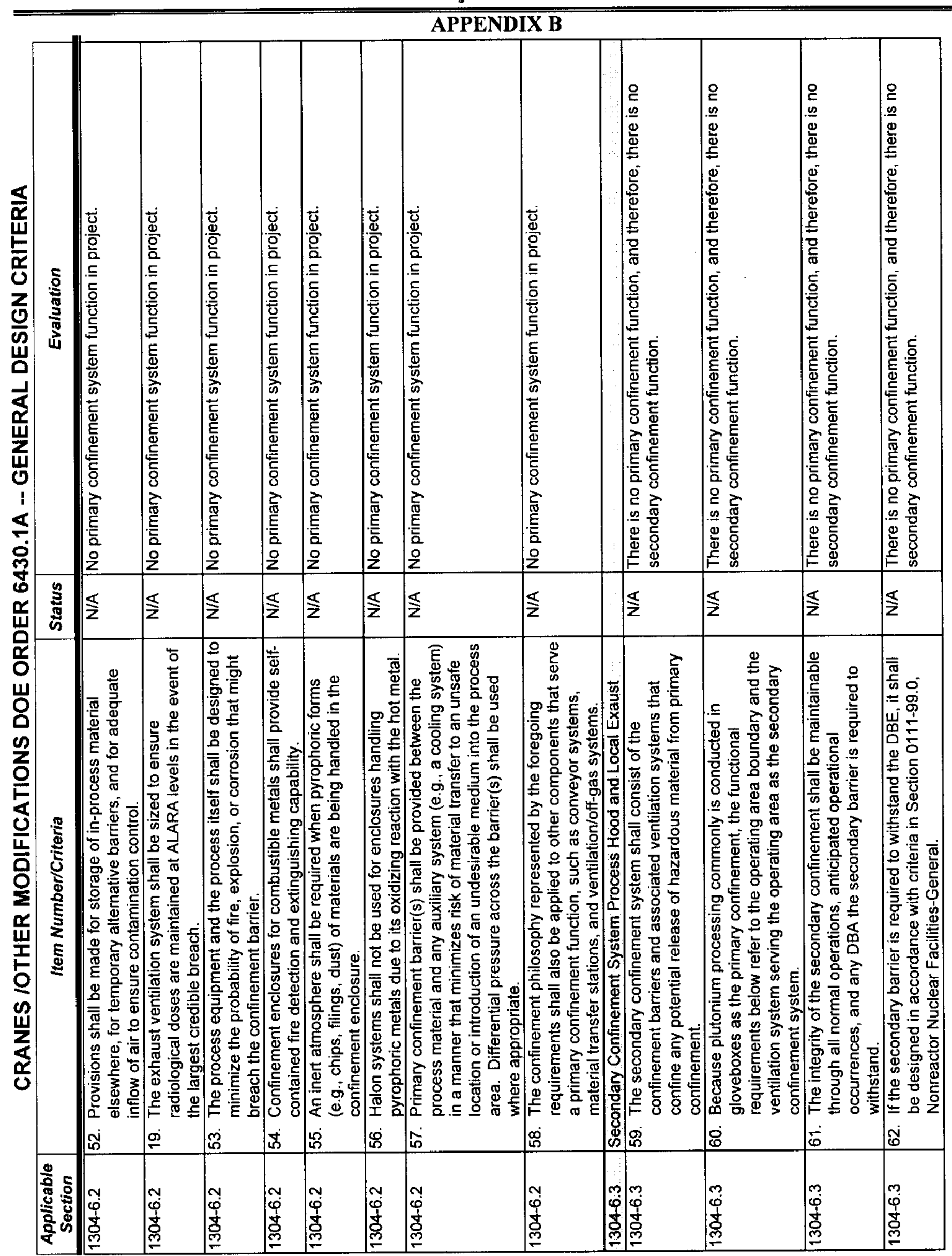




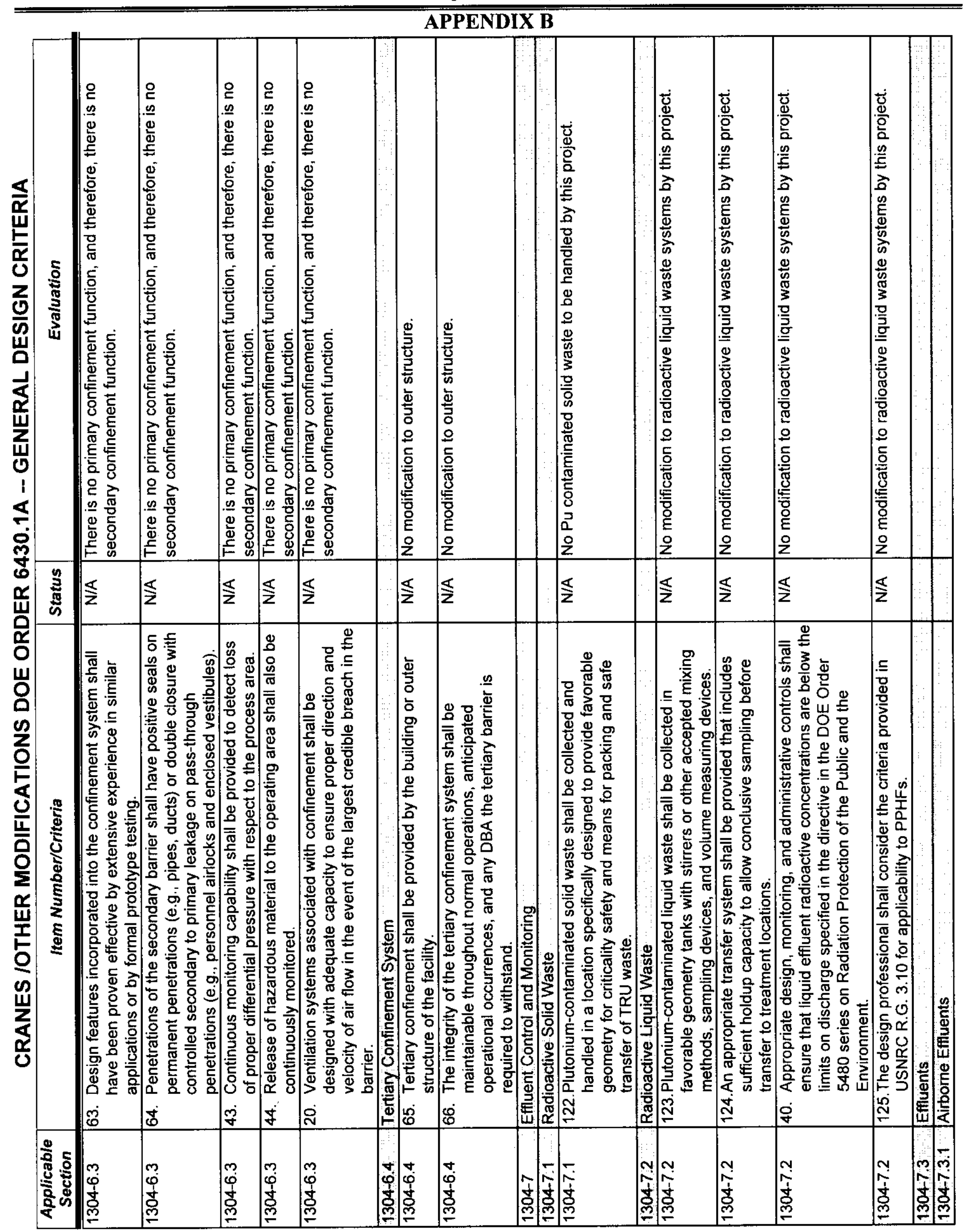


SNF-4775, Rev. 2

Project A.5/A.6

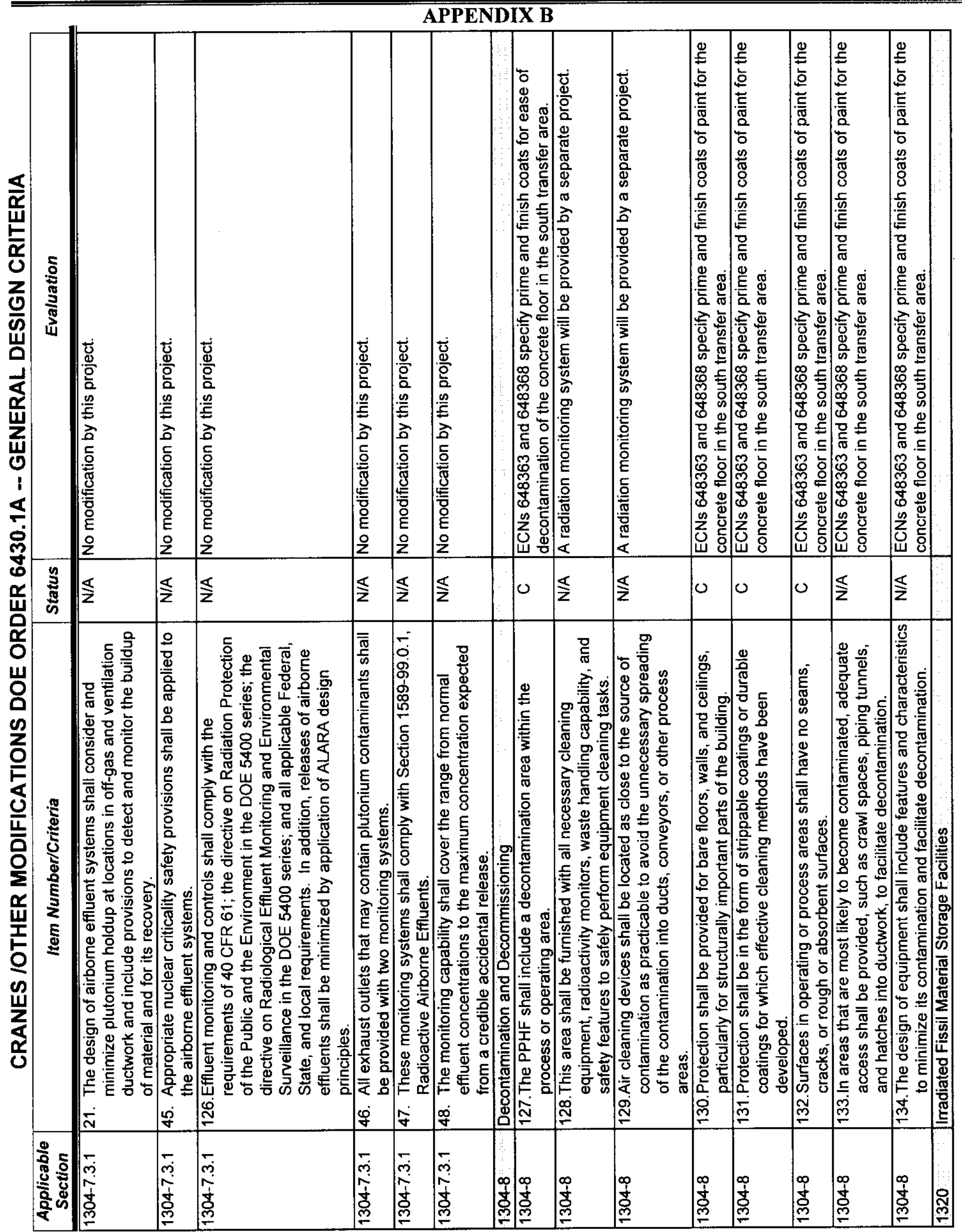


SNF-4775, Rev. 2

Project A.5/A.6

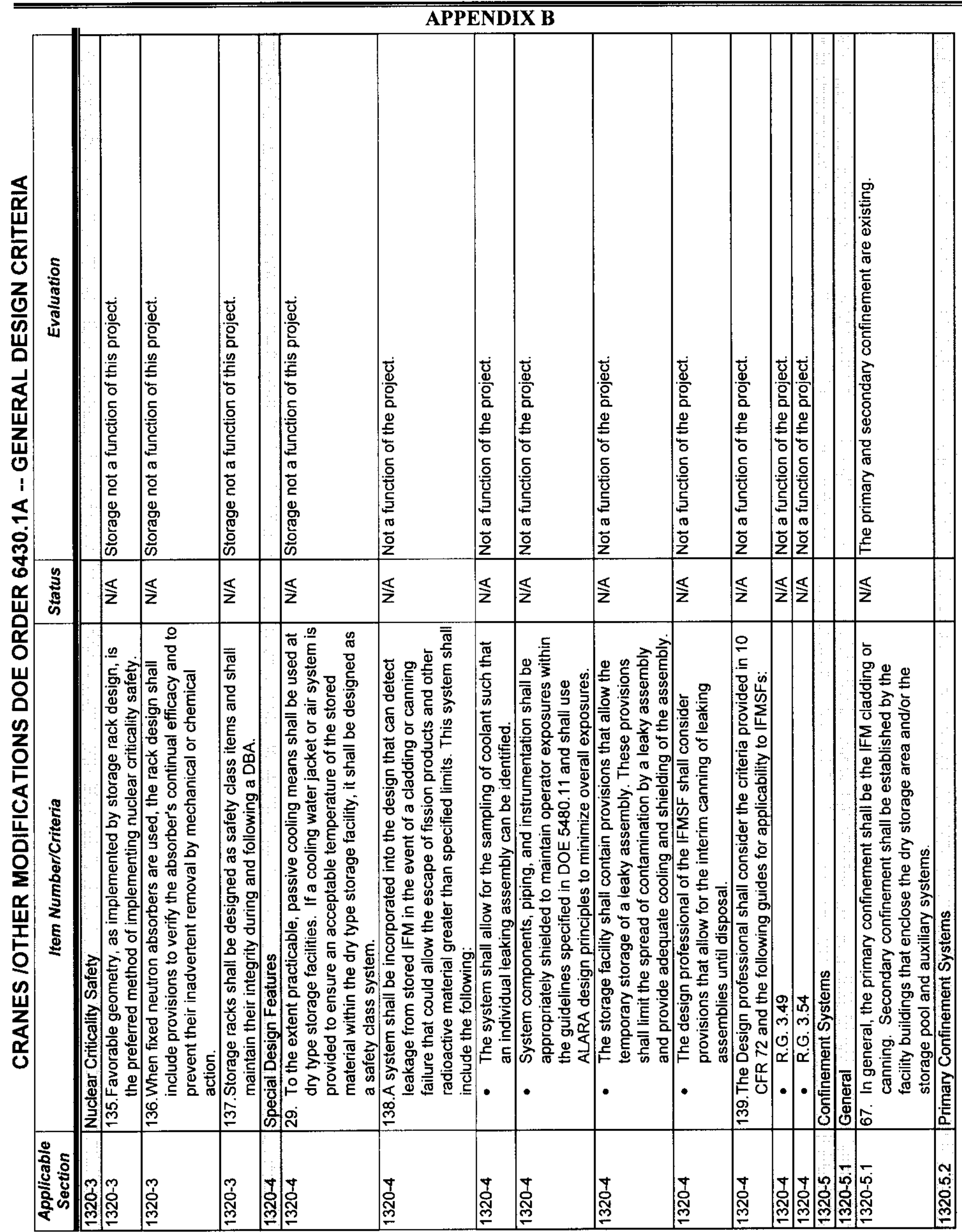


SNF-4775, Rev. 2

Project A.5/A.6

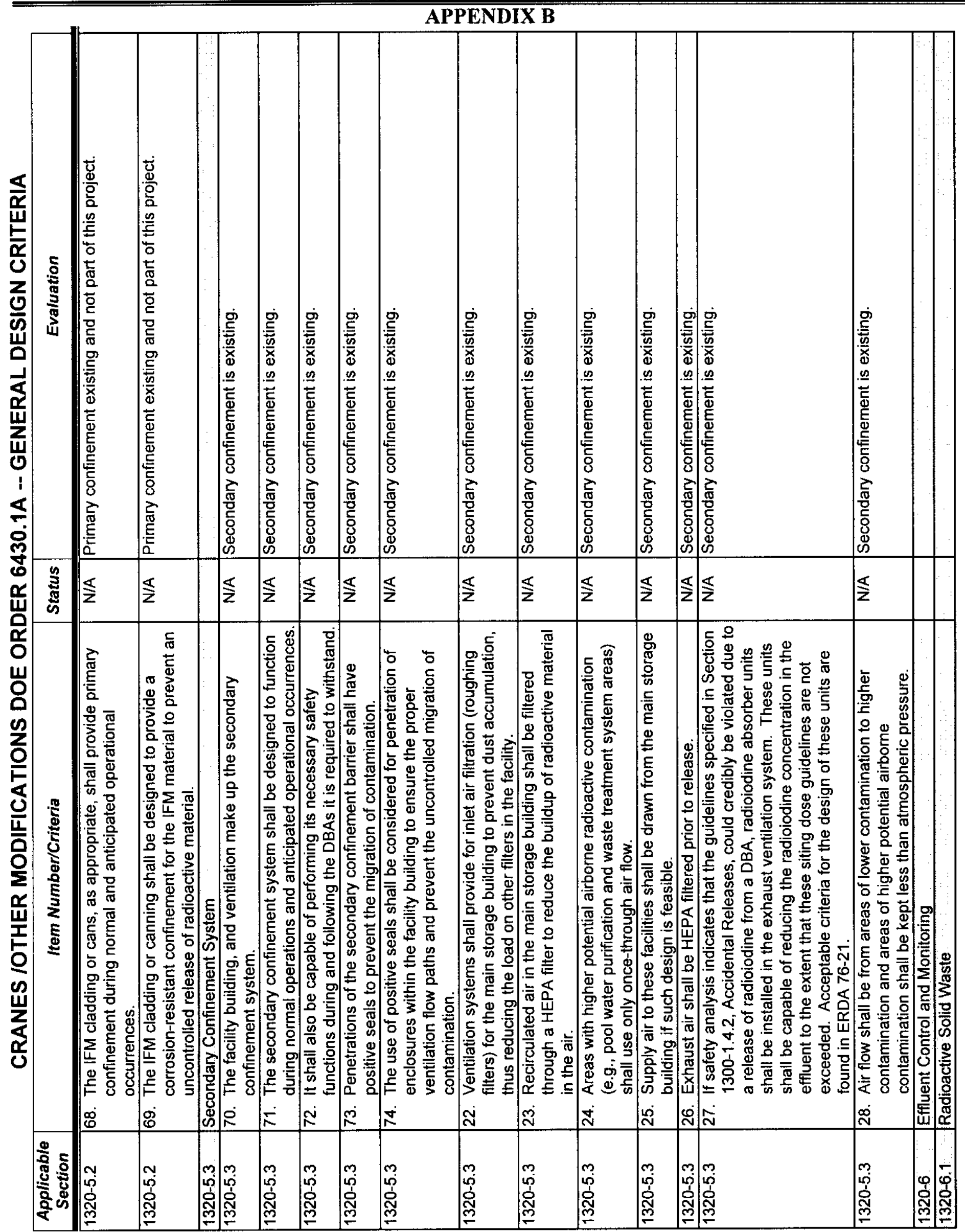


SNF-4775, Rev. 2

Project A.5/A.6

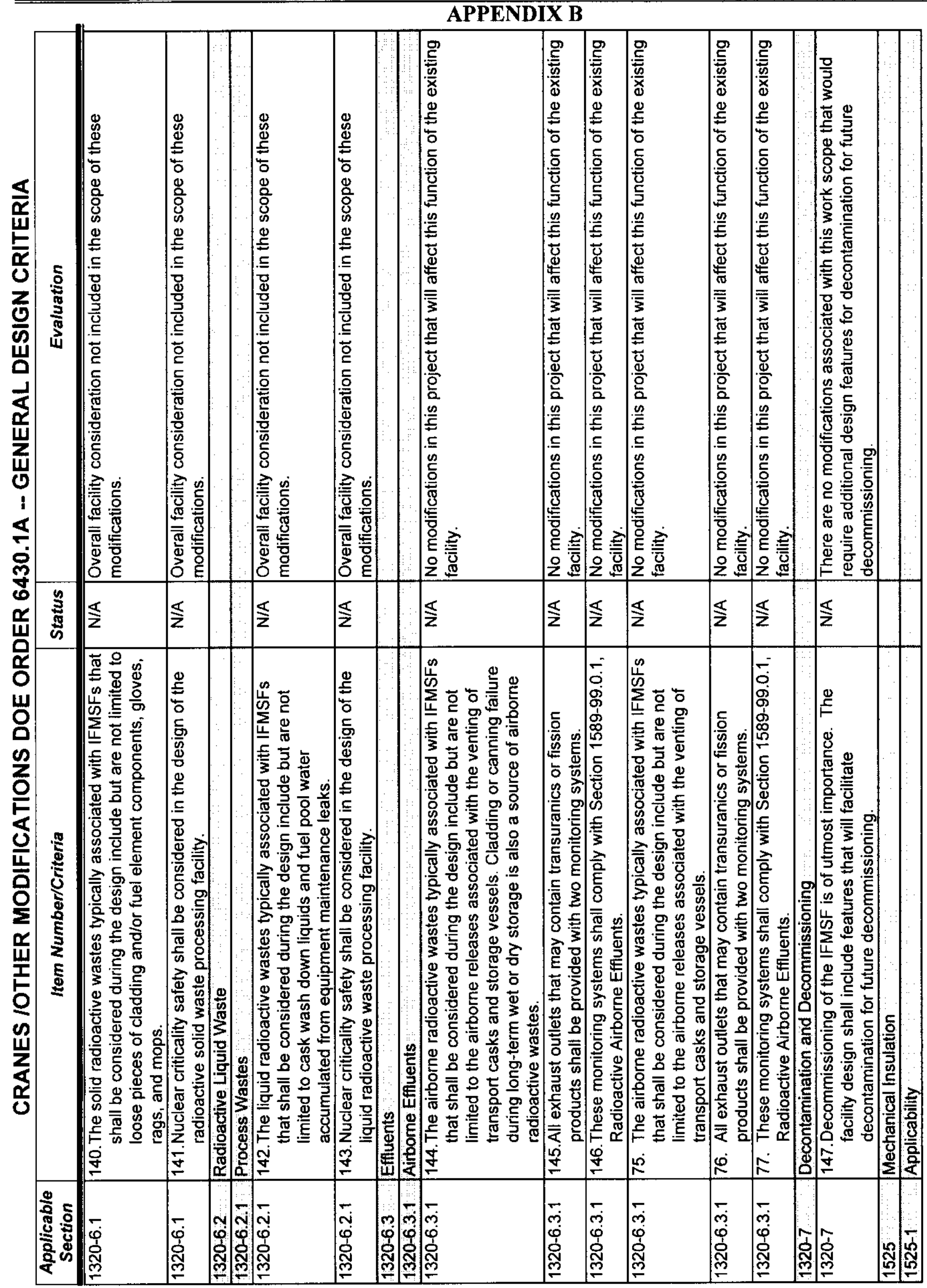


SNF-4775, Rev. 2

Project A.5/A.6

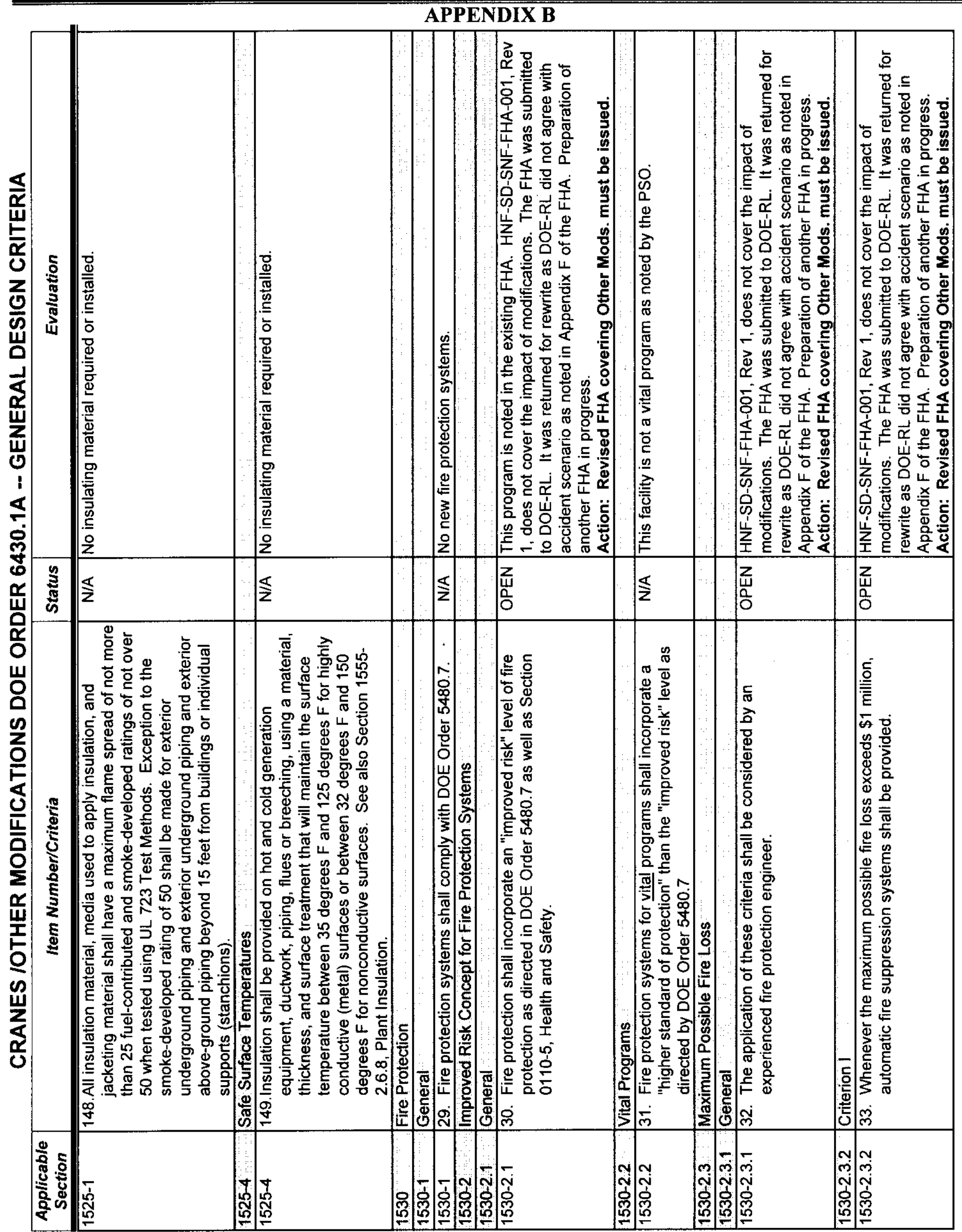


SNF-4775, Rev. 2

Project A.5/A.6

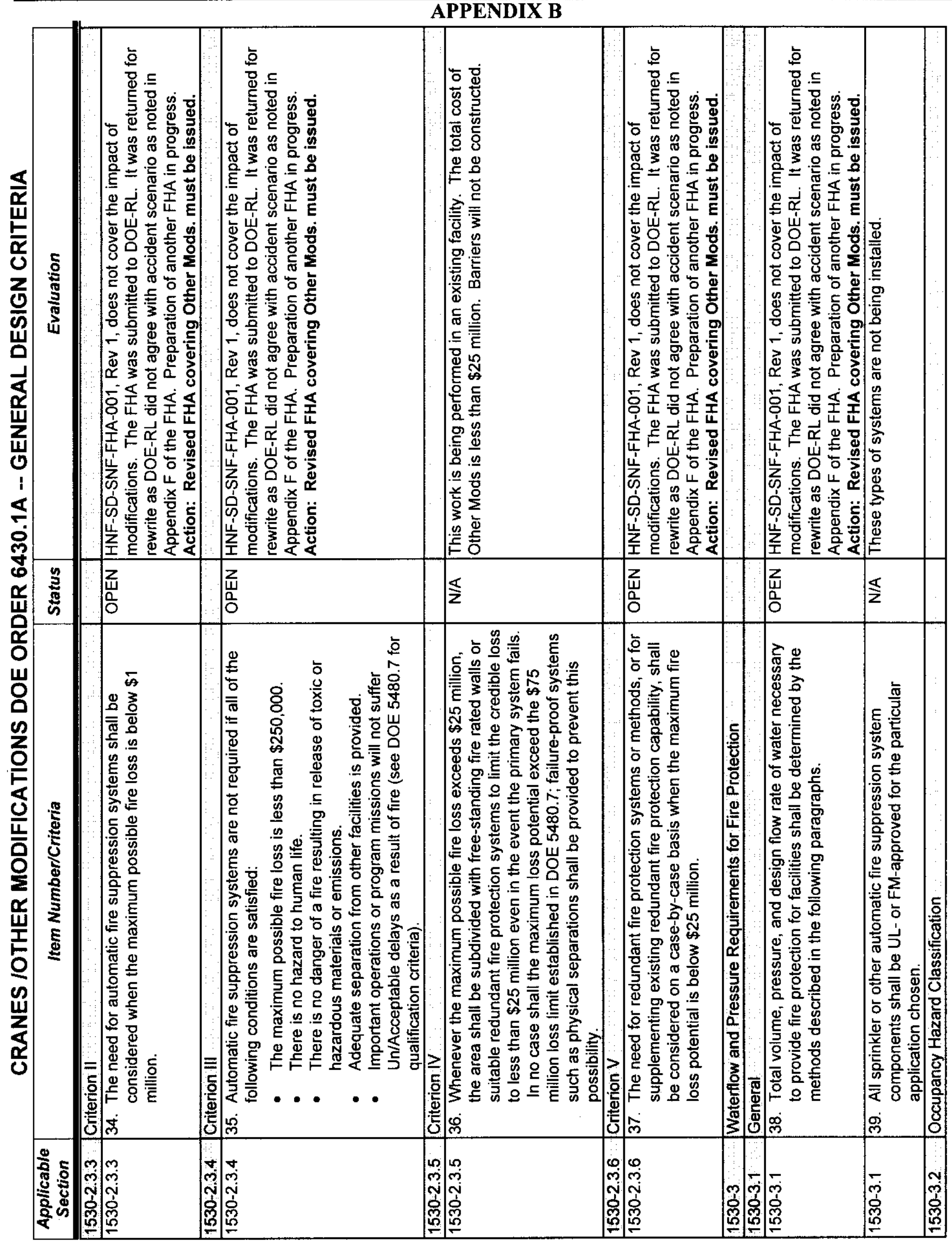


SNF-4775, Rev. 2

Project A.5/A.6

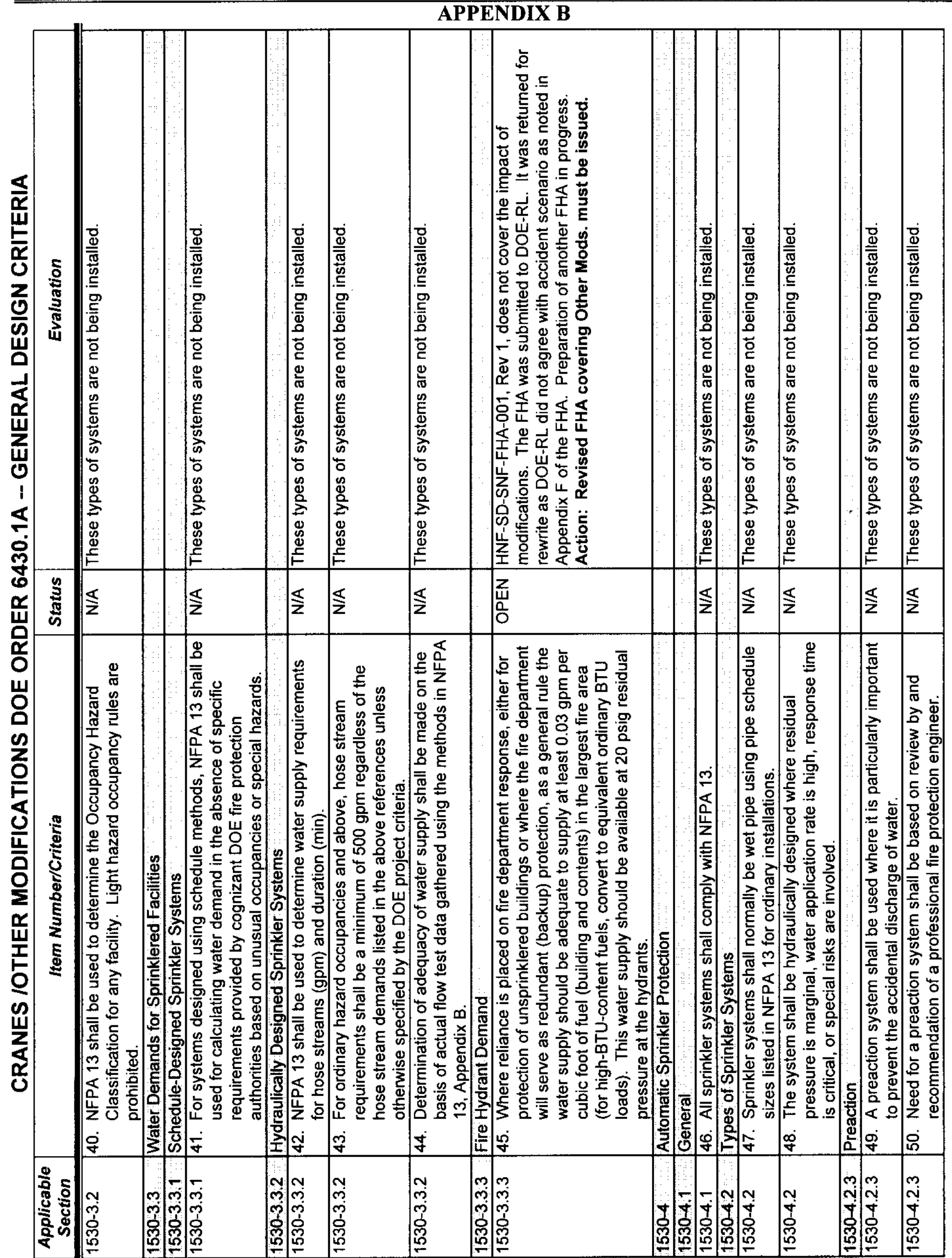


SNF-4775, Rev. 2

Project A.5/A.6

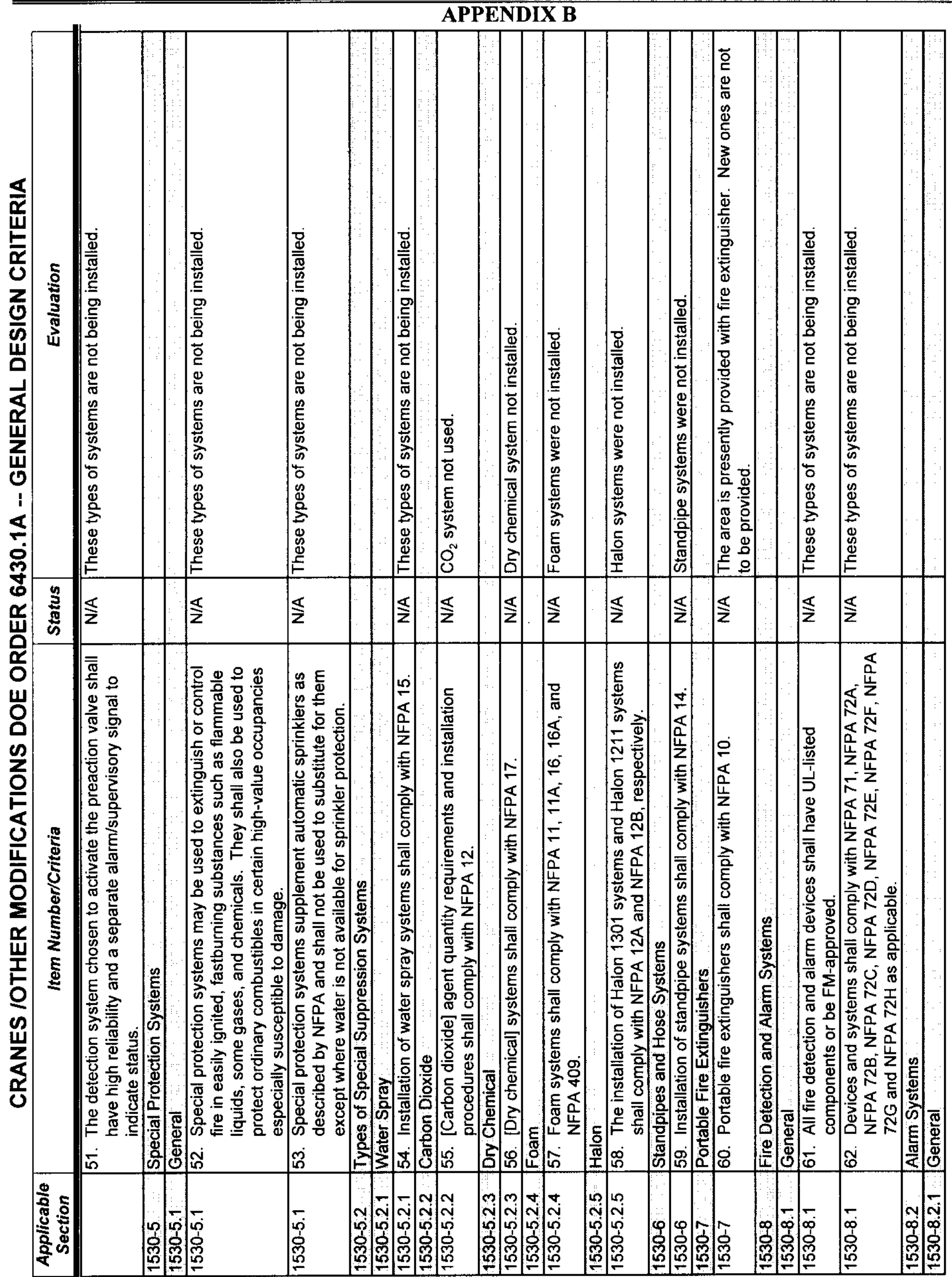




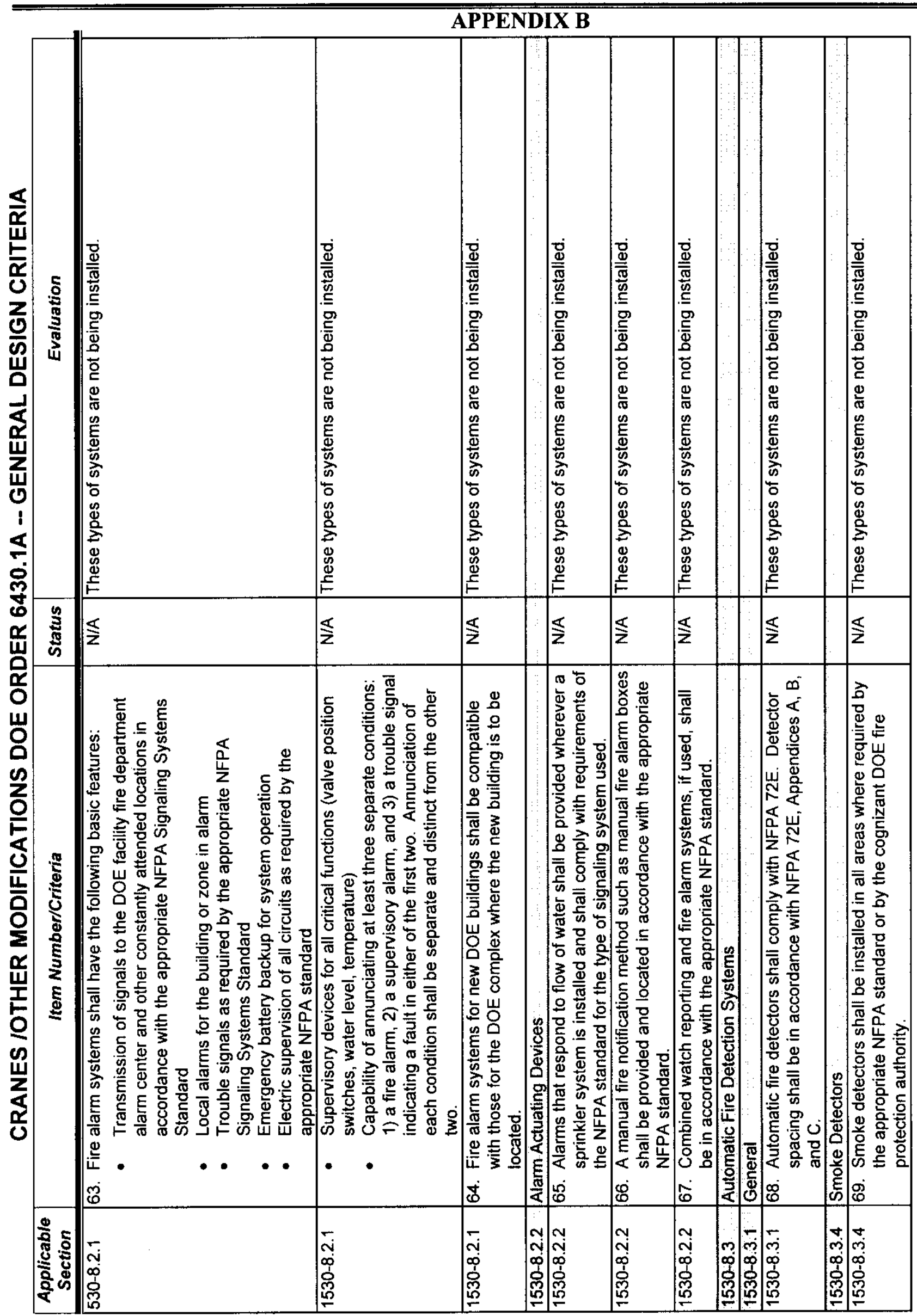


SNF-4775, Rev. 2

Project A.5/A.6

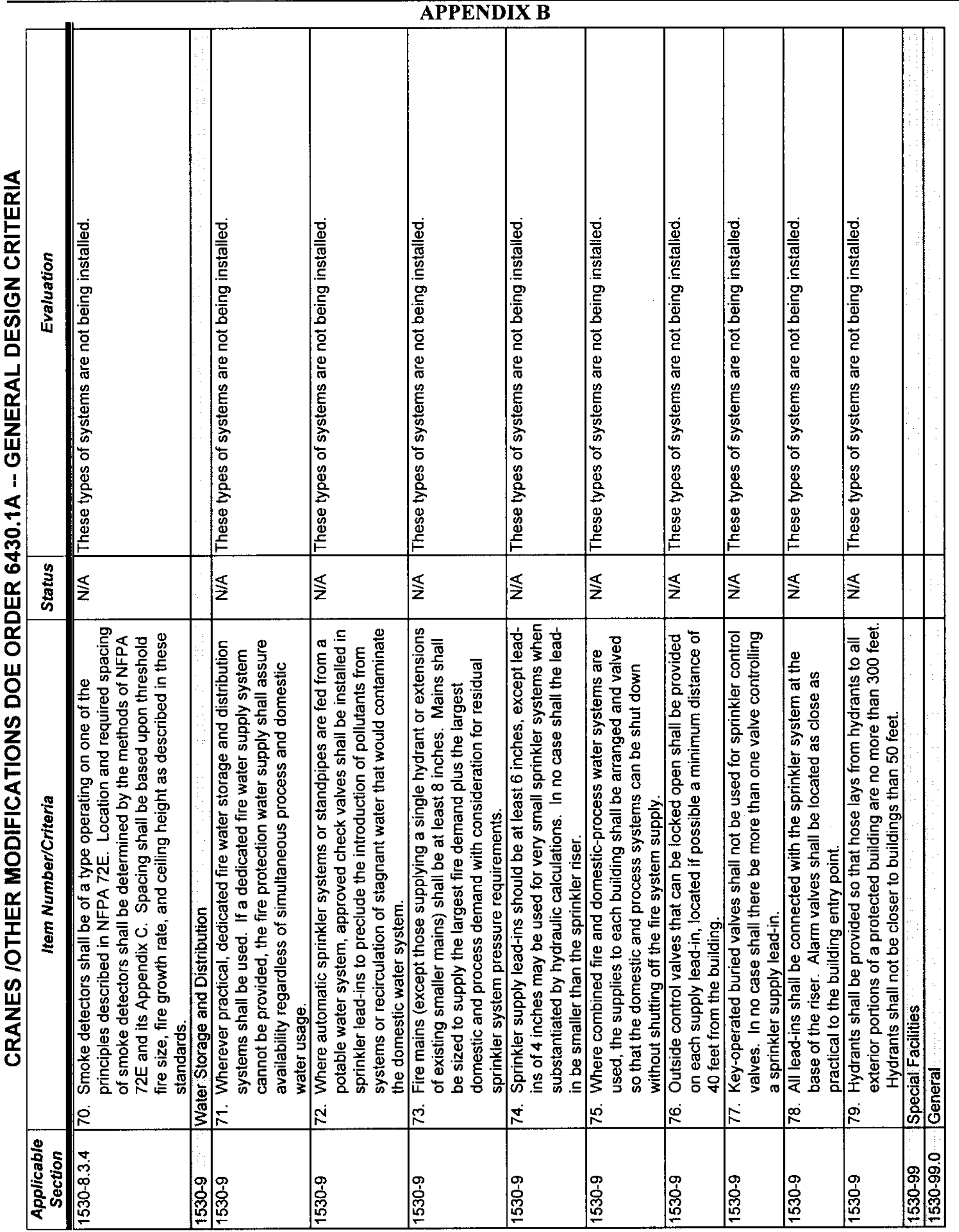


SNF-4775, Rev. 2

Project A.5/A.6

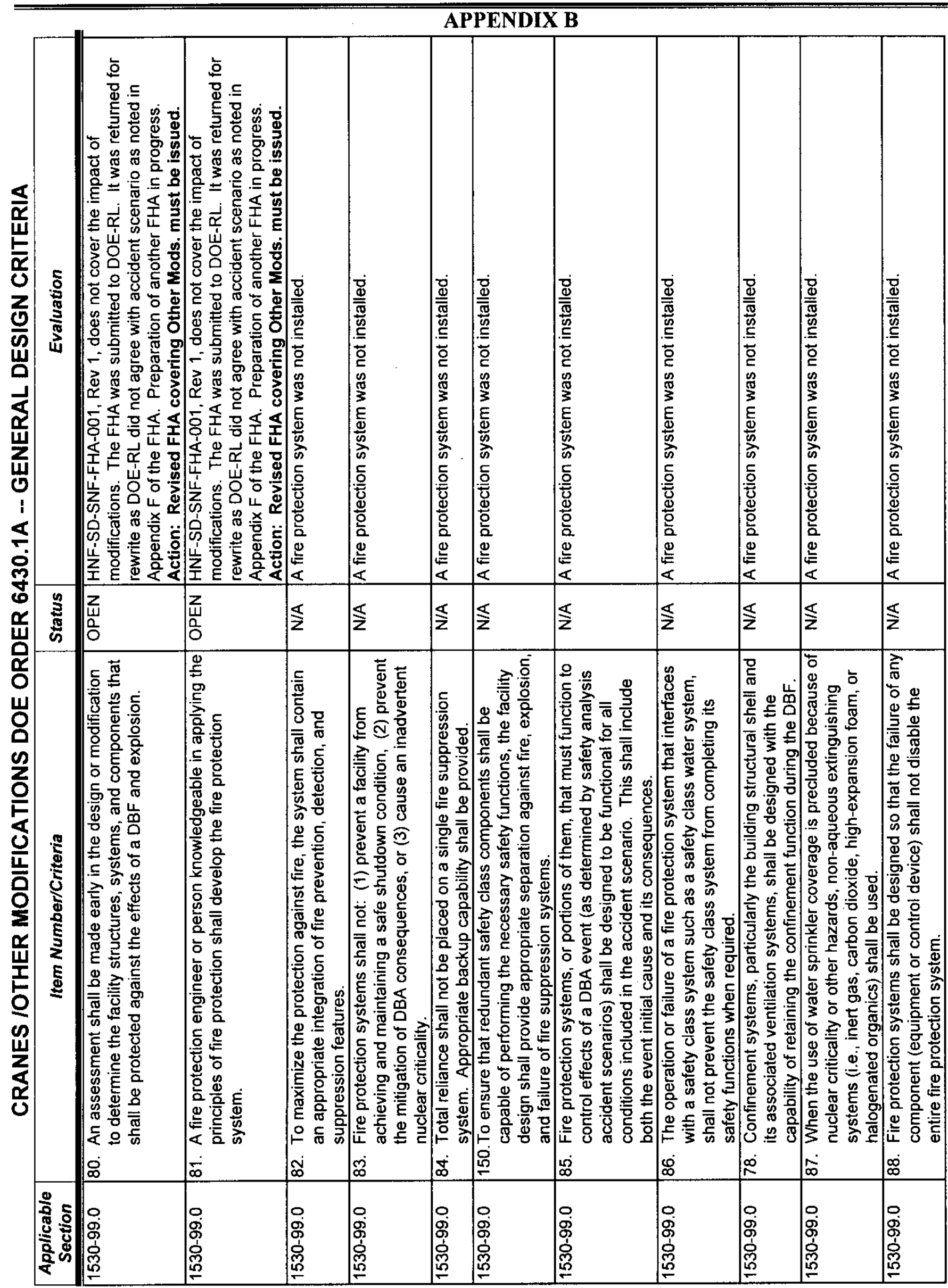


SNF-4775, Rev. 2

Project A.5/A.6

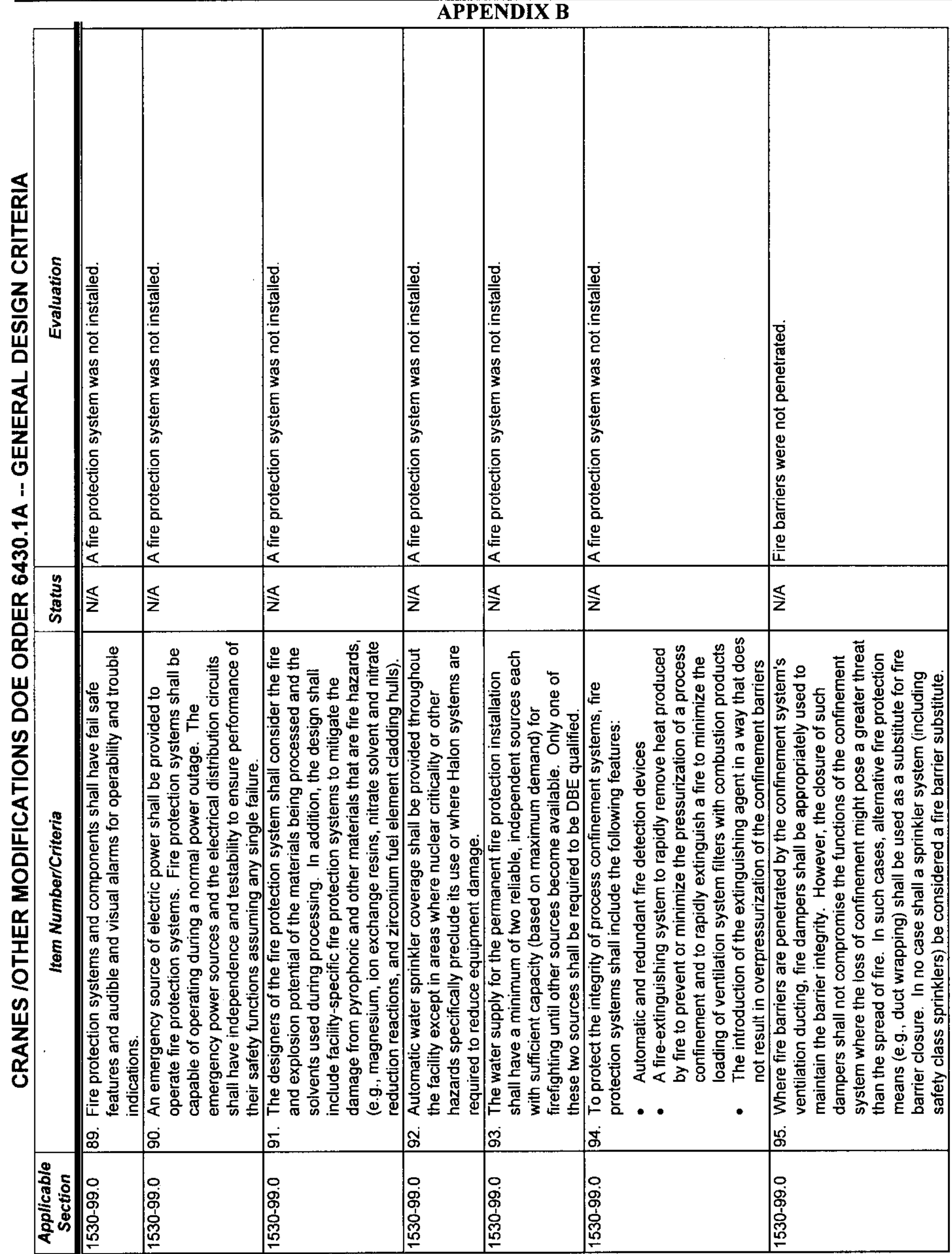

Page B-72 


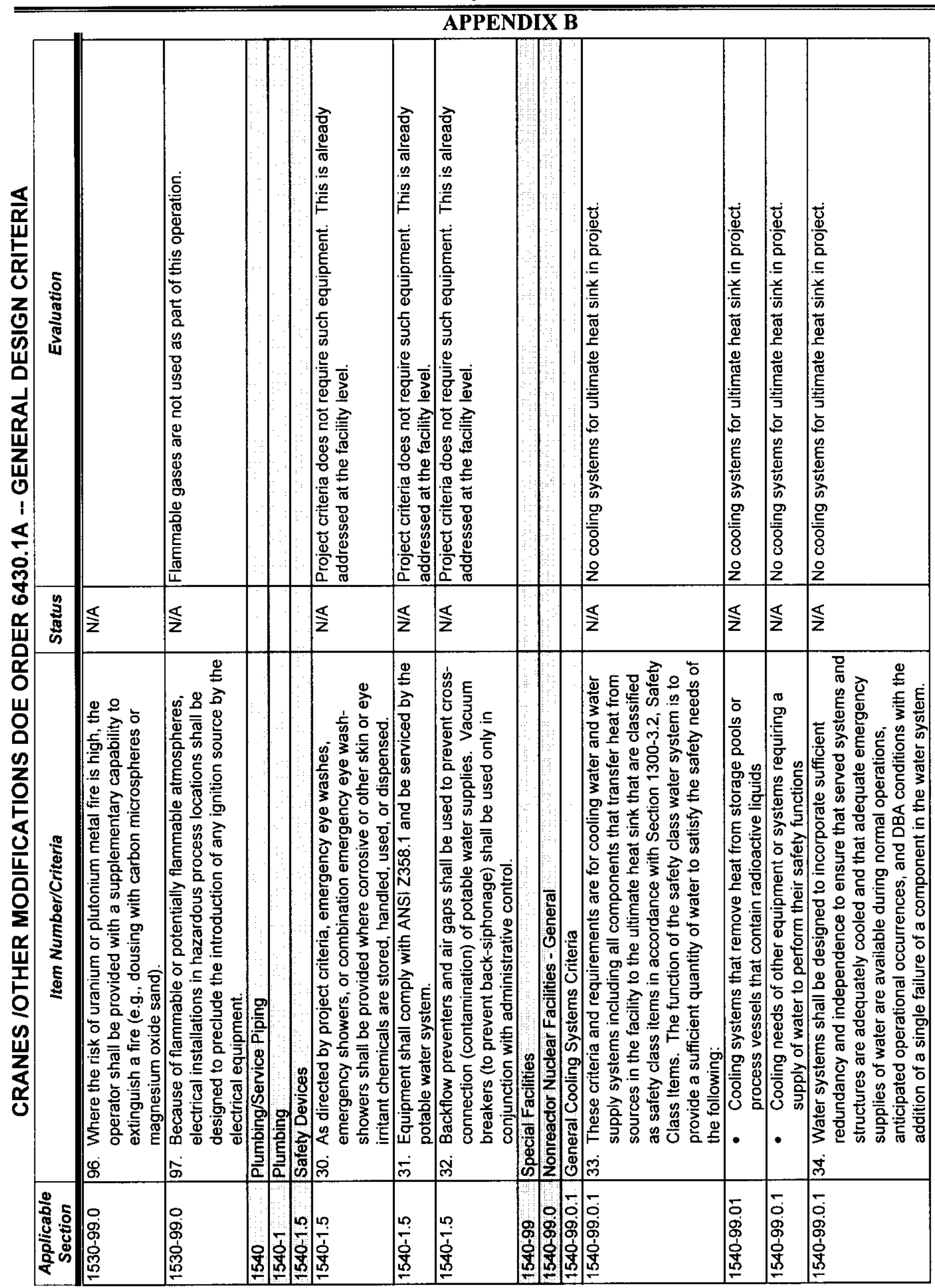




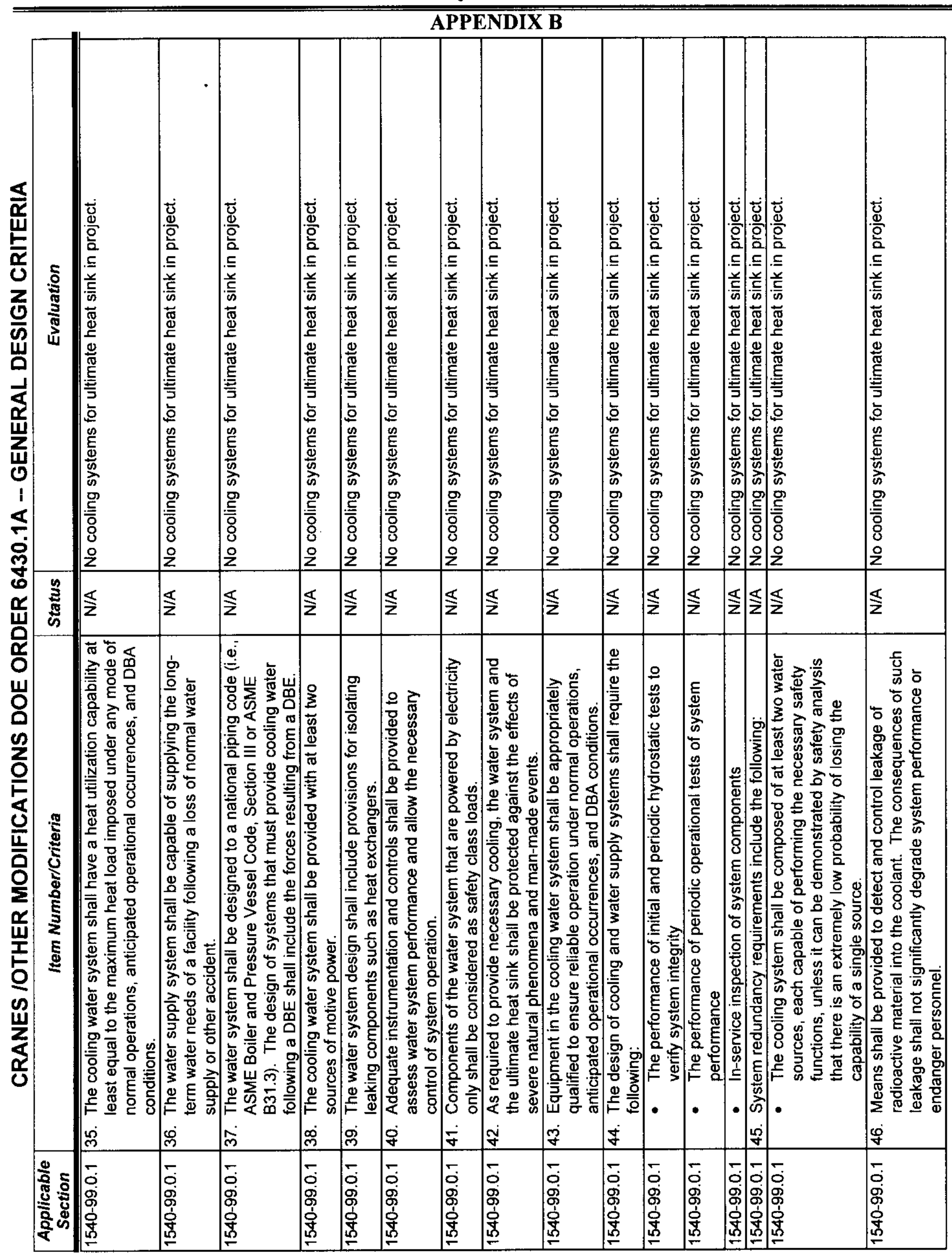


SNF-4775, Rev. 2

Project A.5/A.6

APPENDIX B

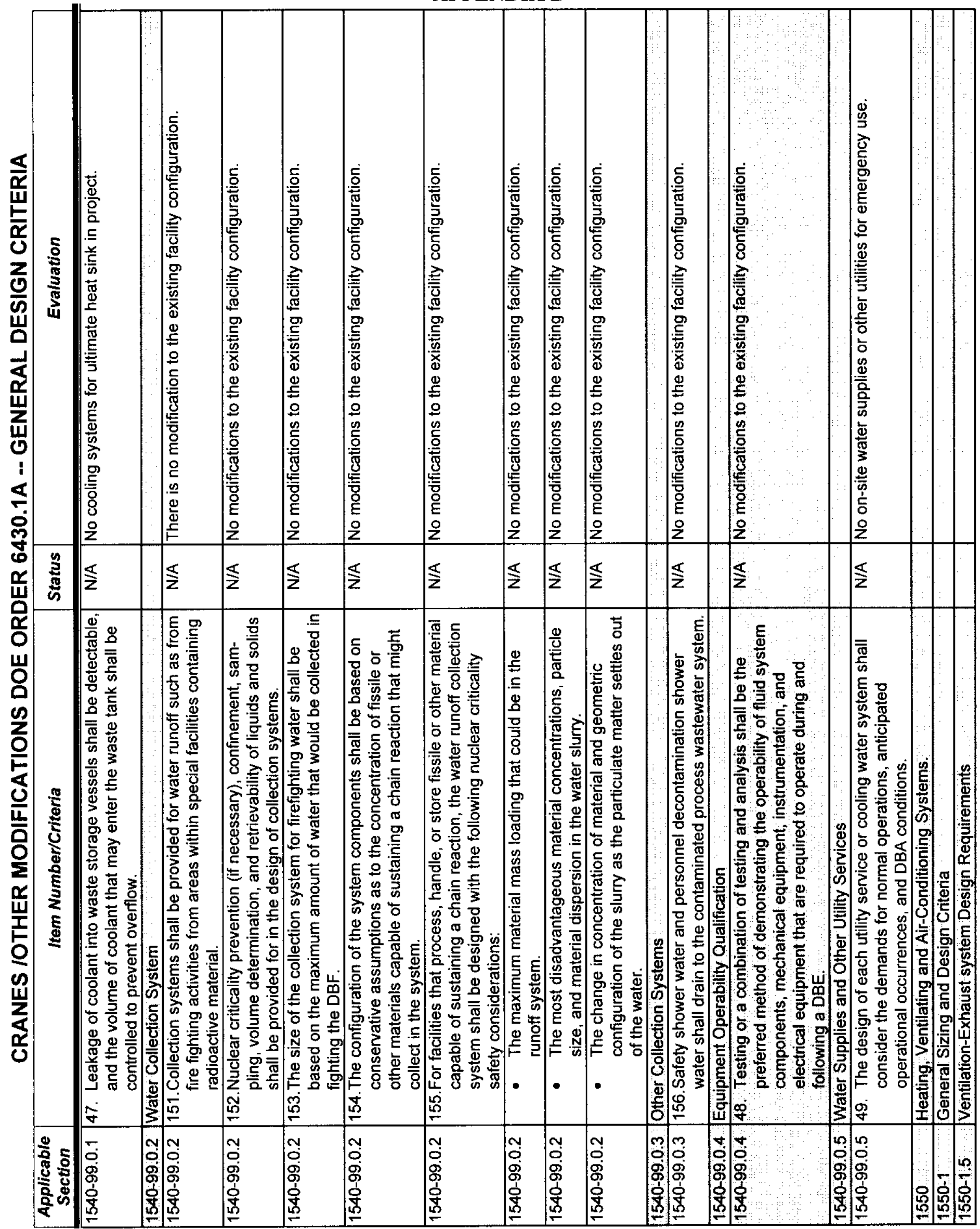


SNF-4775, Rev. 2

Project A.5/A.6

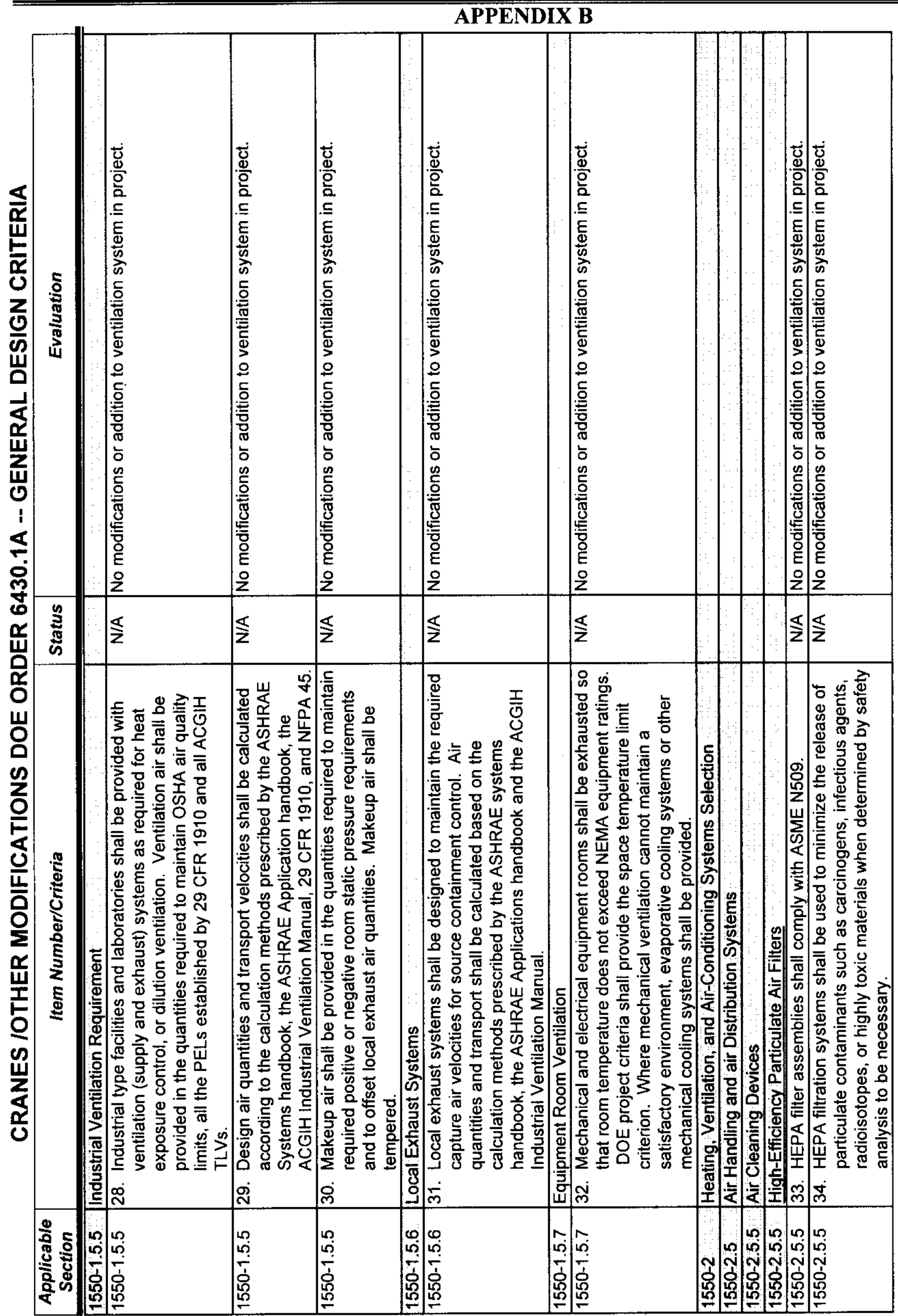


SNF-4775, Rev. 2

Project A.5/A.6

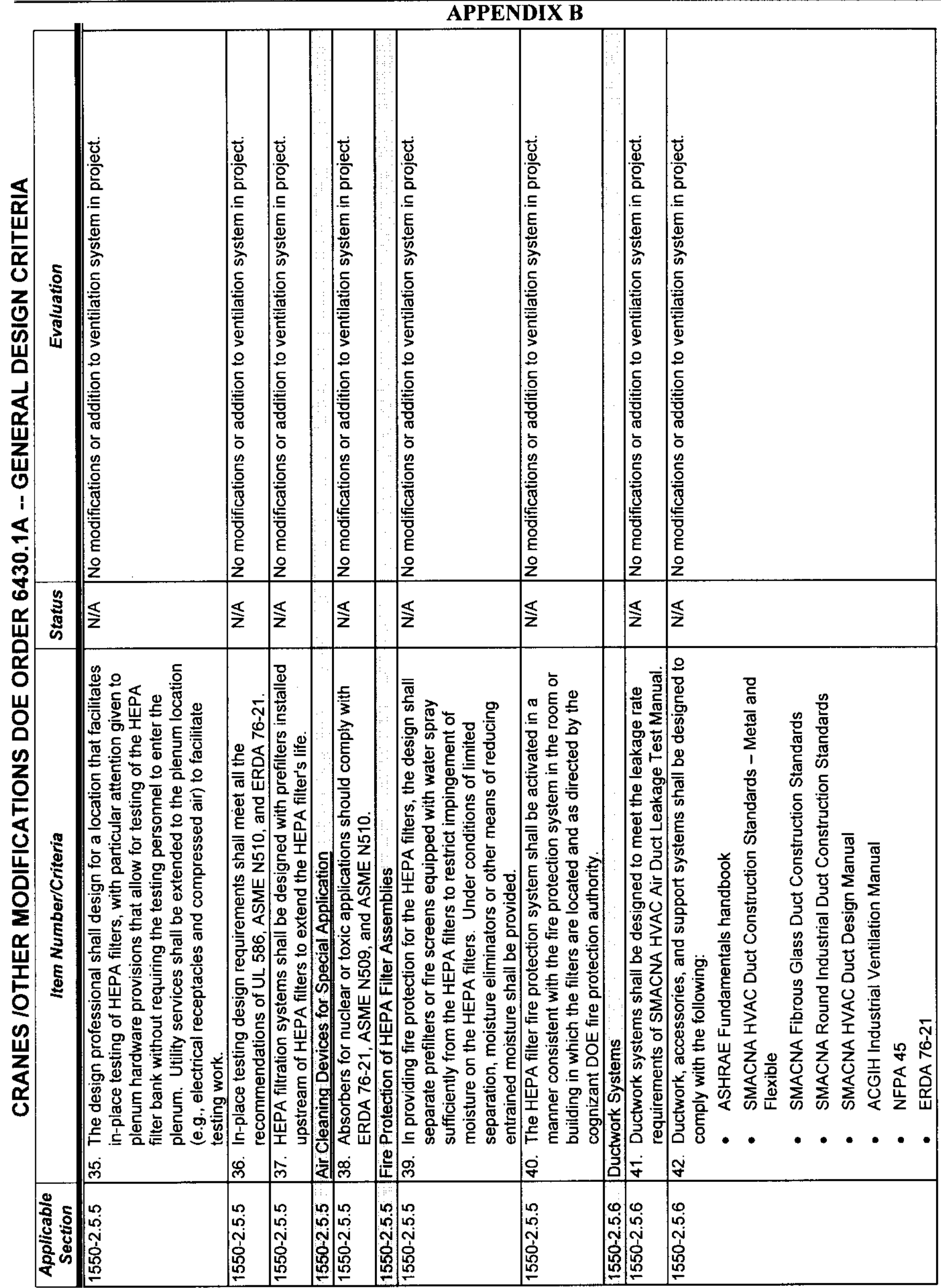


SNF-4775, Rev. 2

Project A.5/A.6

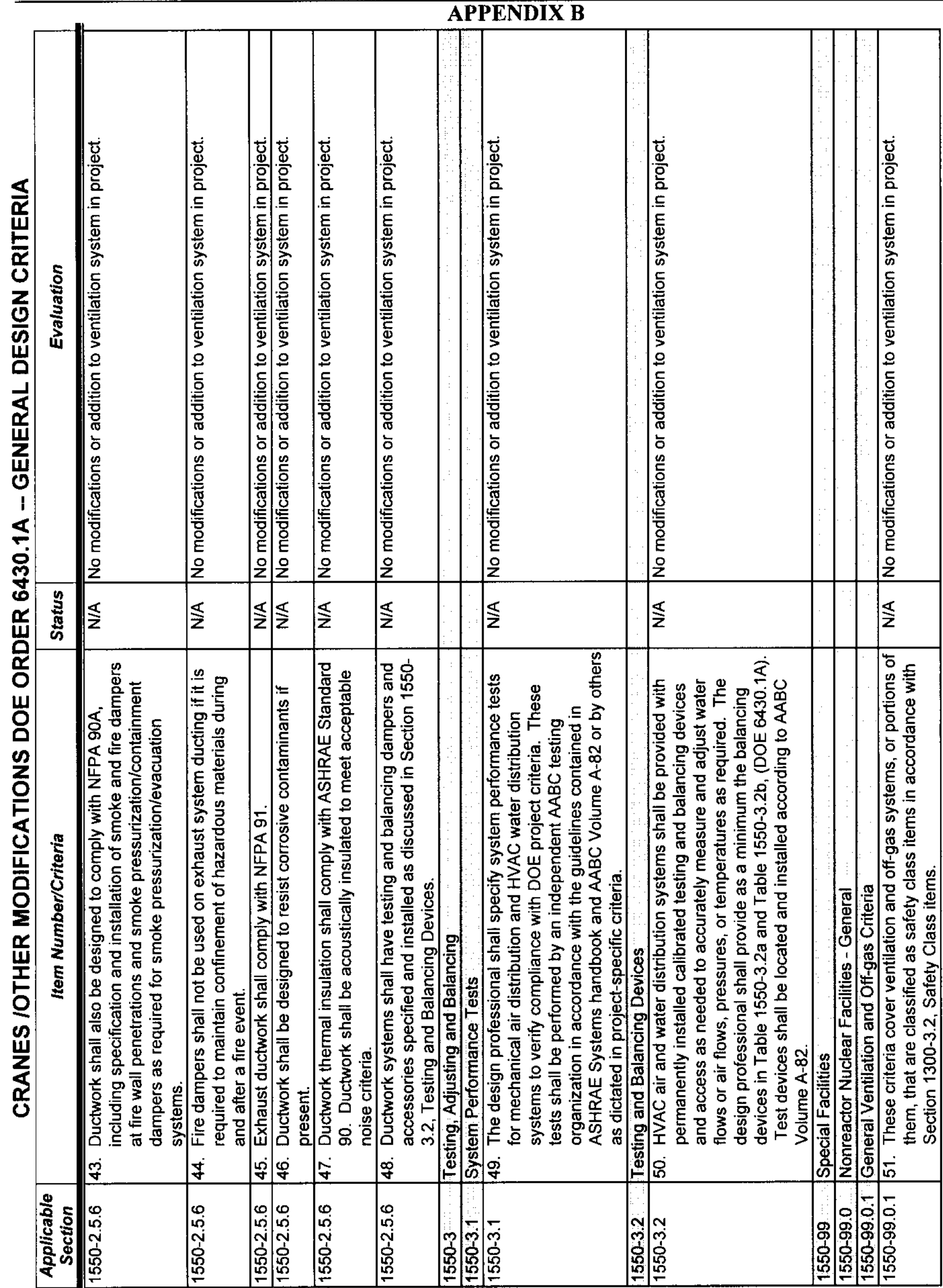


SNF-4775, Rev. 2

Project A.5/A.6

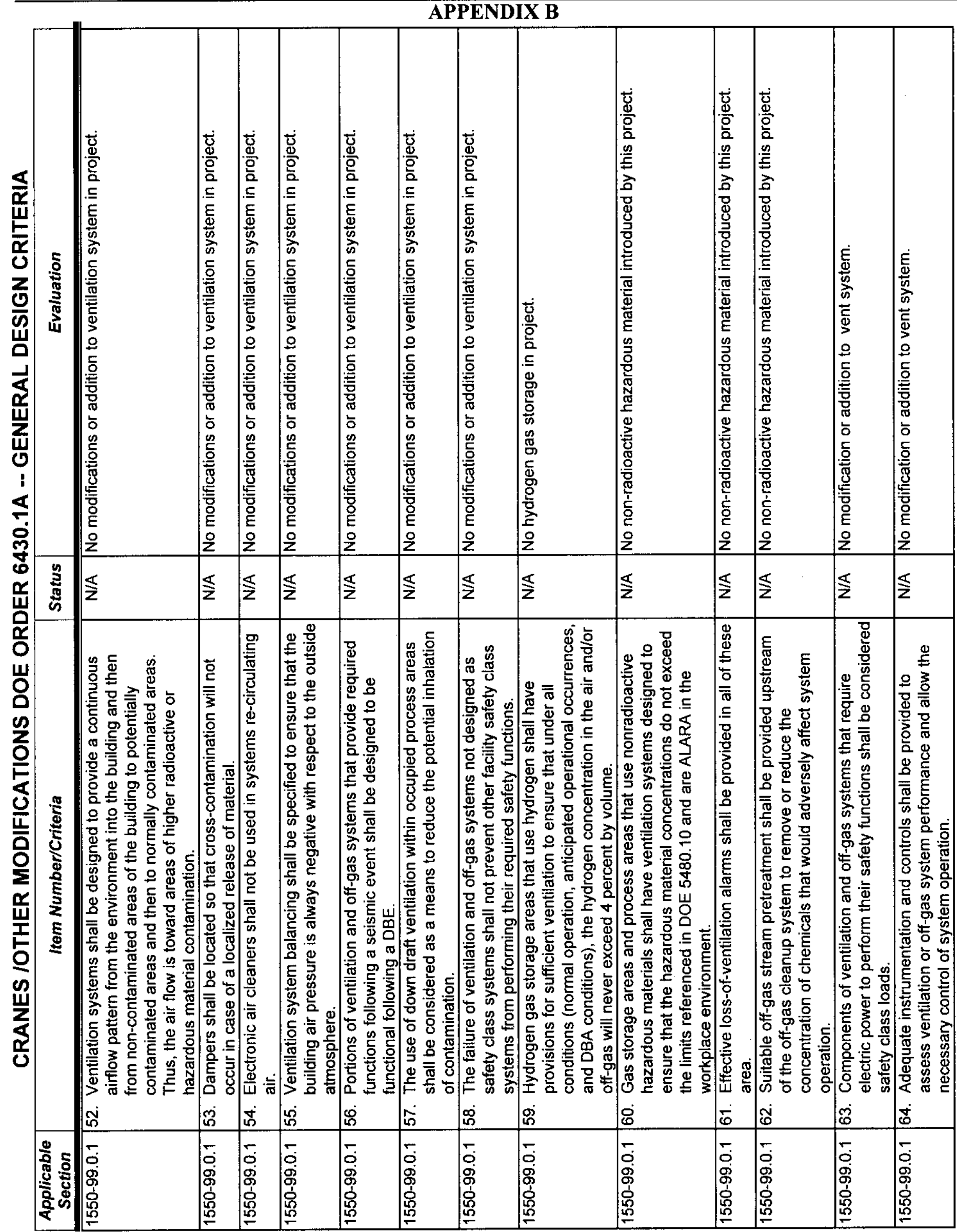


SNF-4775, Rev. 2

Project A.5/A.6

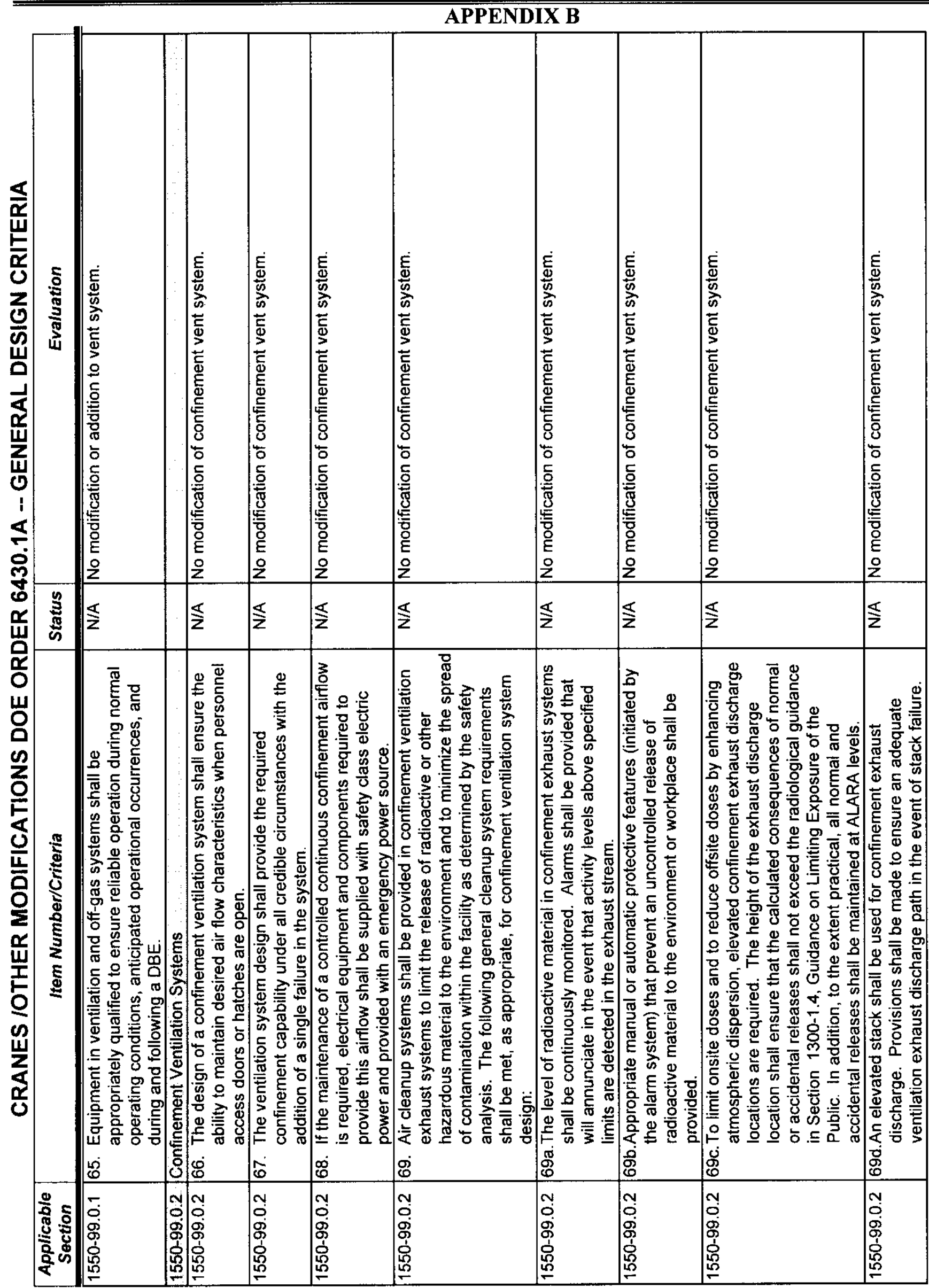


SNF-4775, Rev. 2

Project A.5/A.6

APPENDIX B

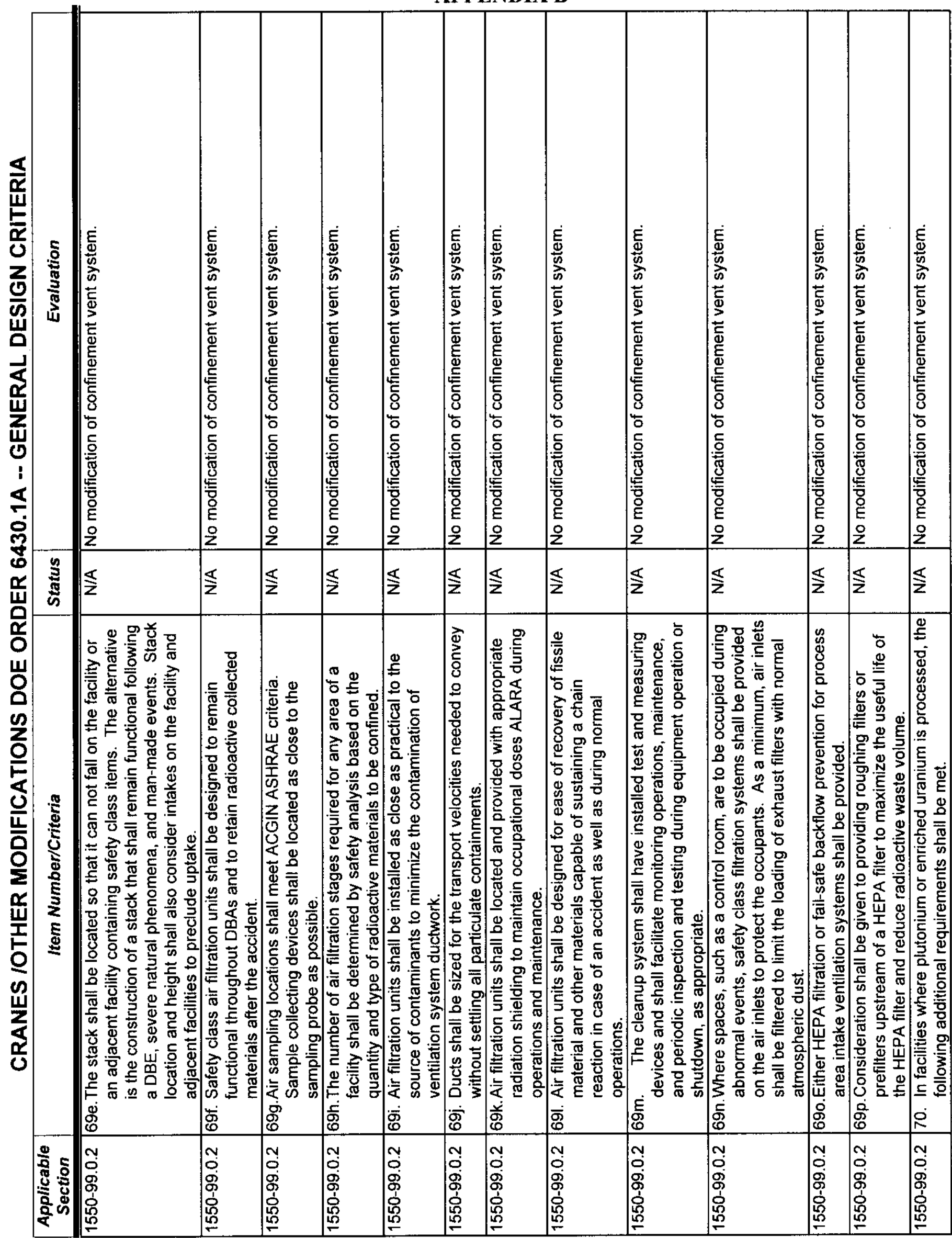


SNF-4775, Rev. 2

Project A.5/A.6

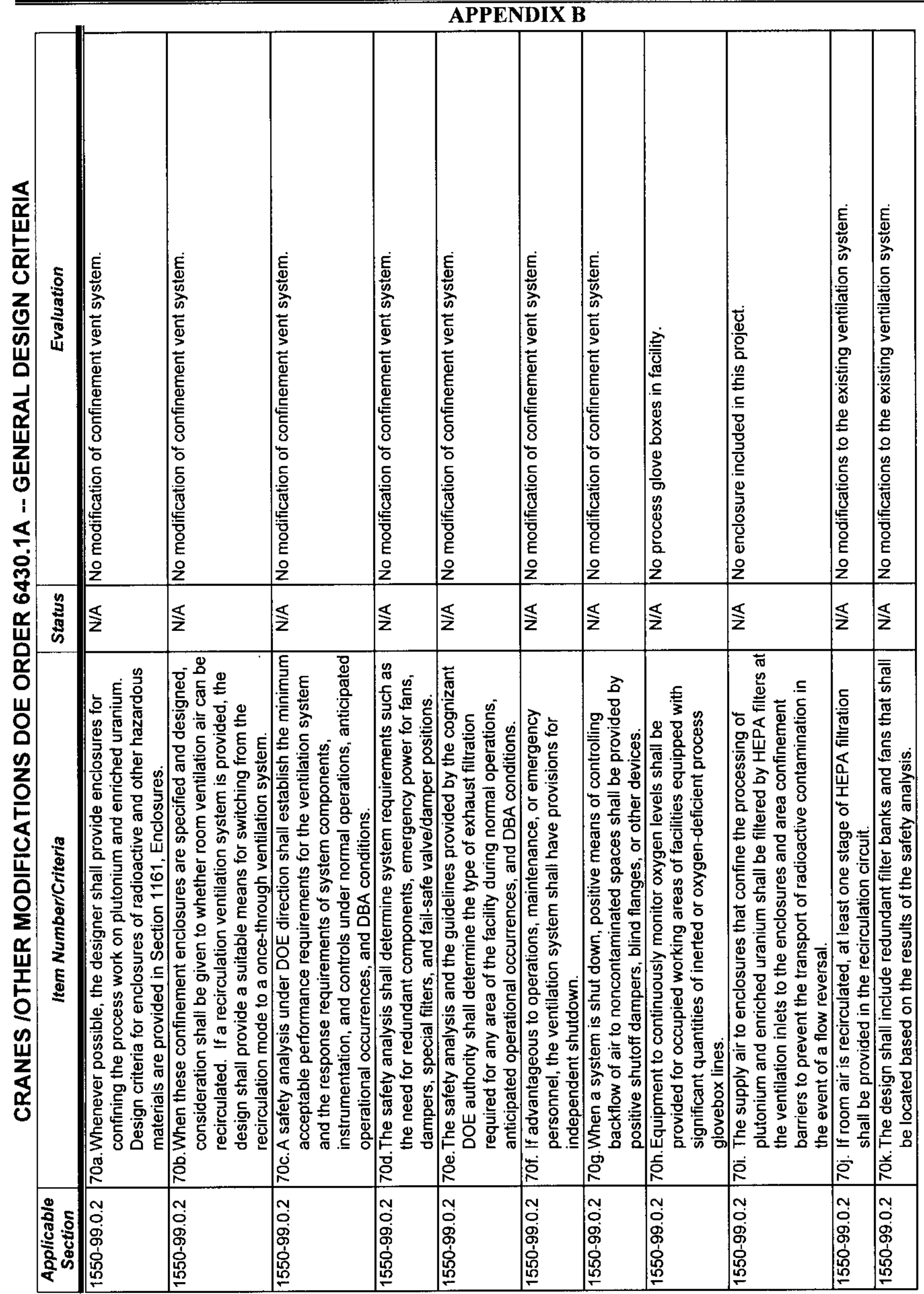

Page B-82 


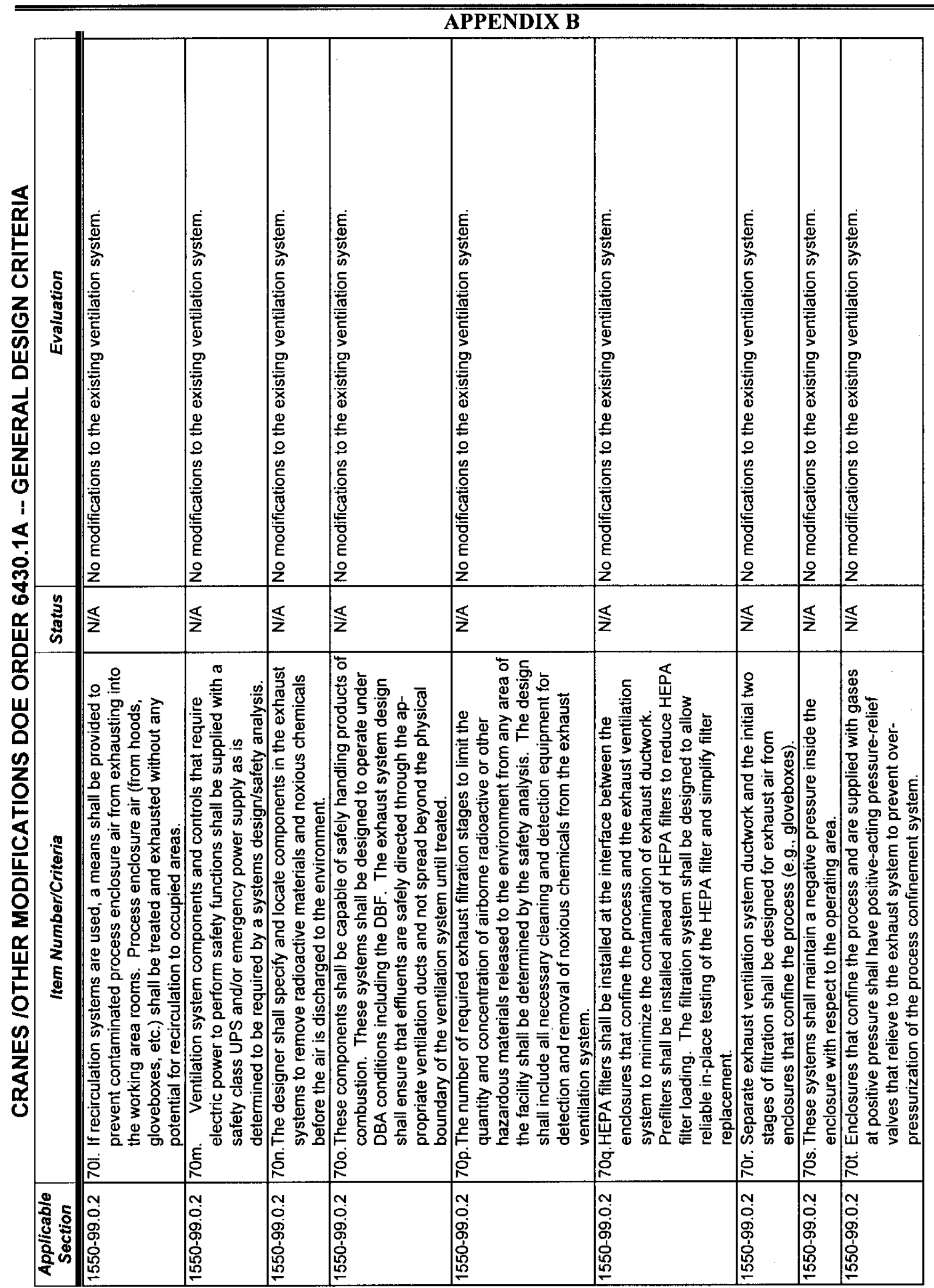


SNF-4775, Rev. 2

Project A.5/A.6

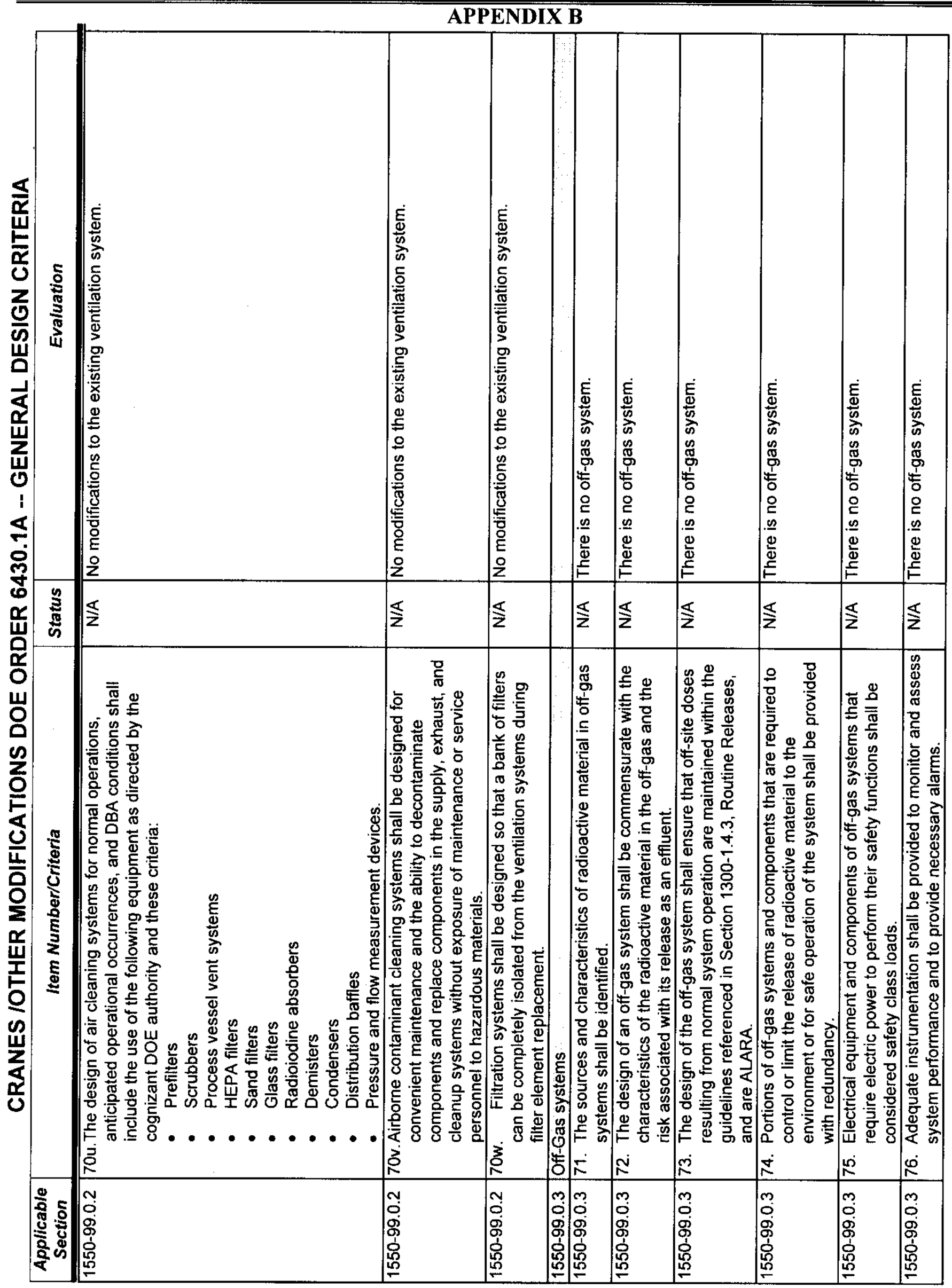

Page B-84 


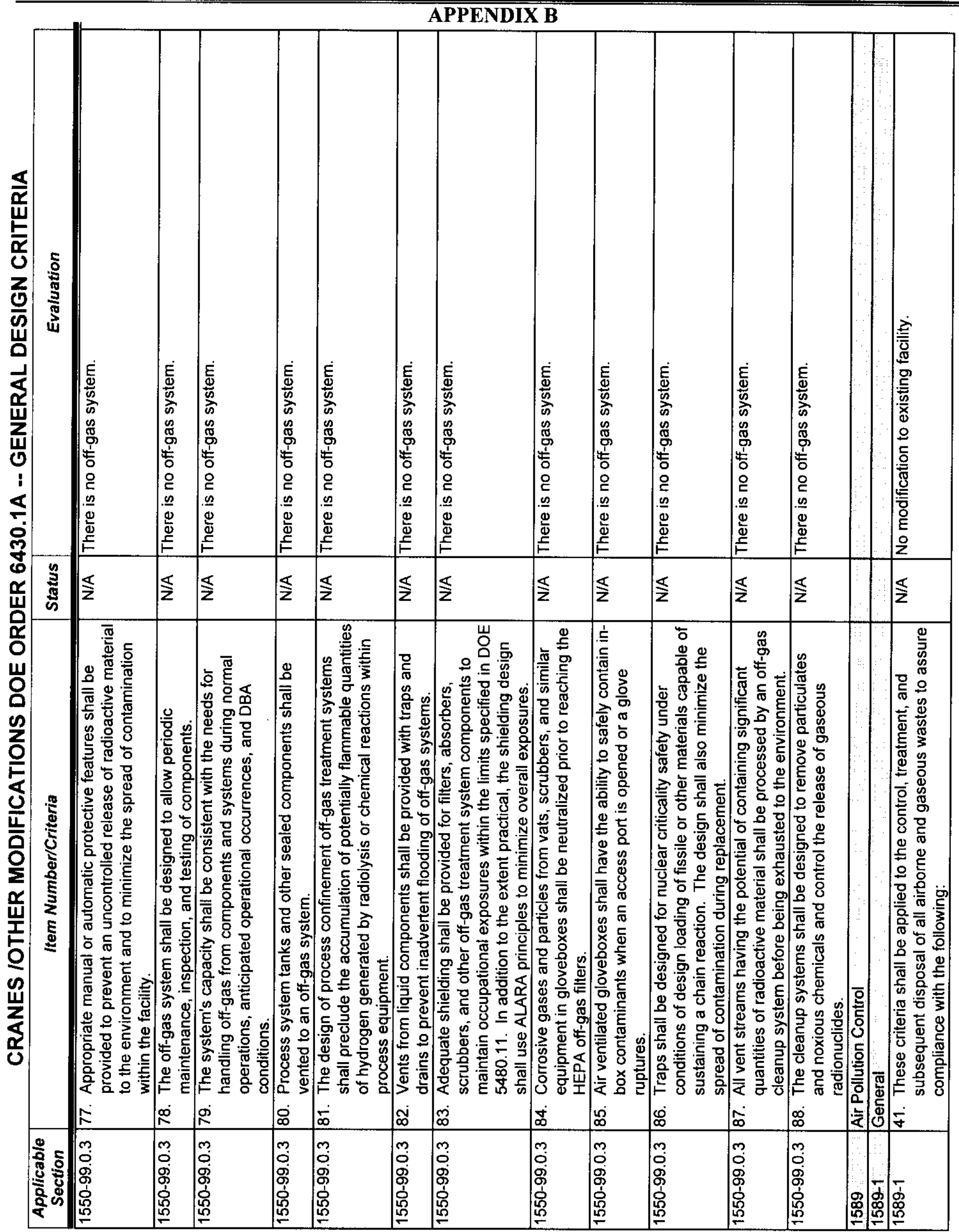




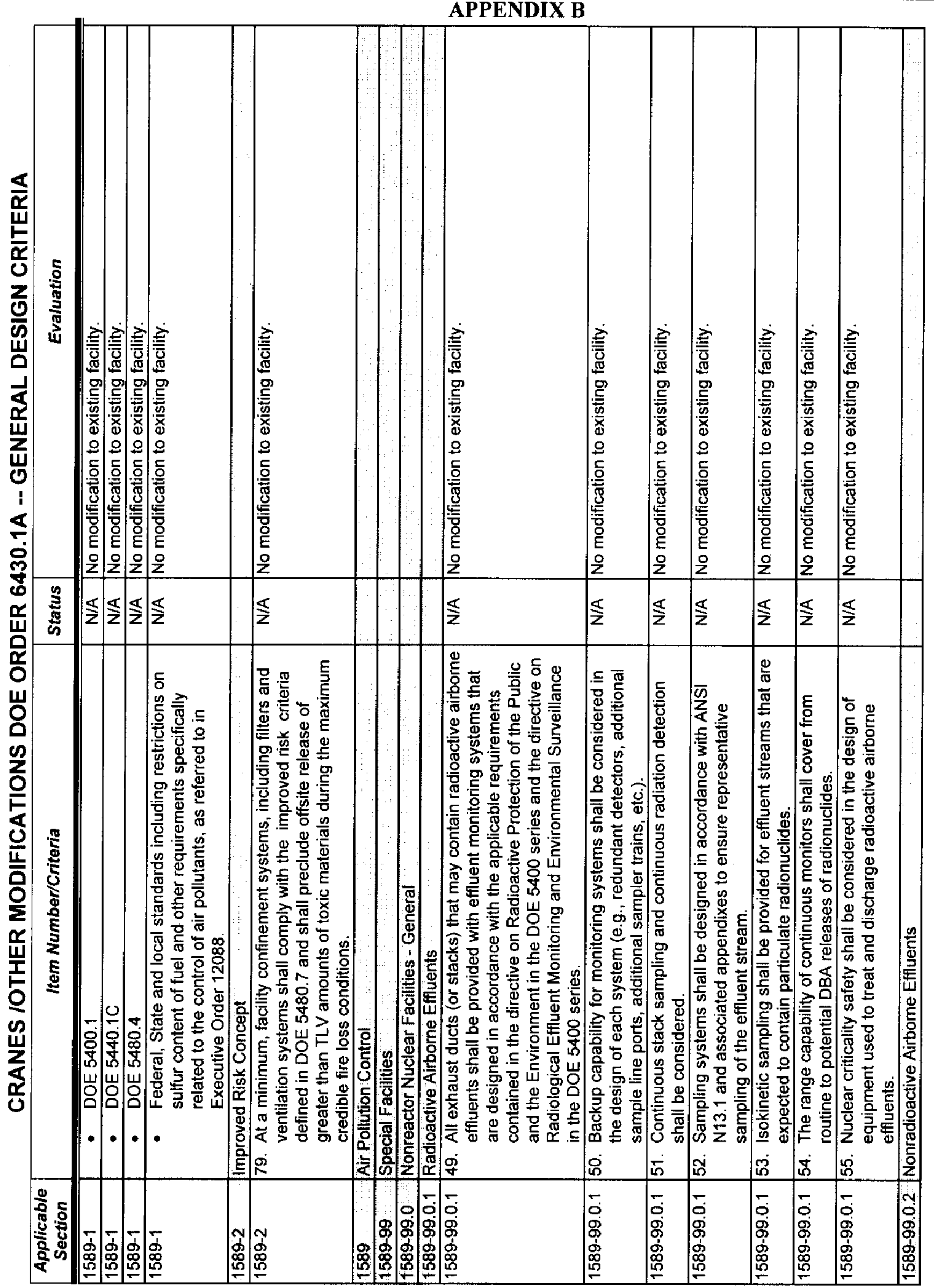


SNF-4775, Rev. 2

Project A.5/A.6

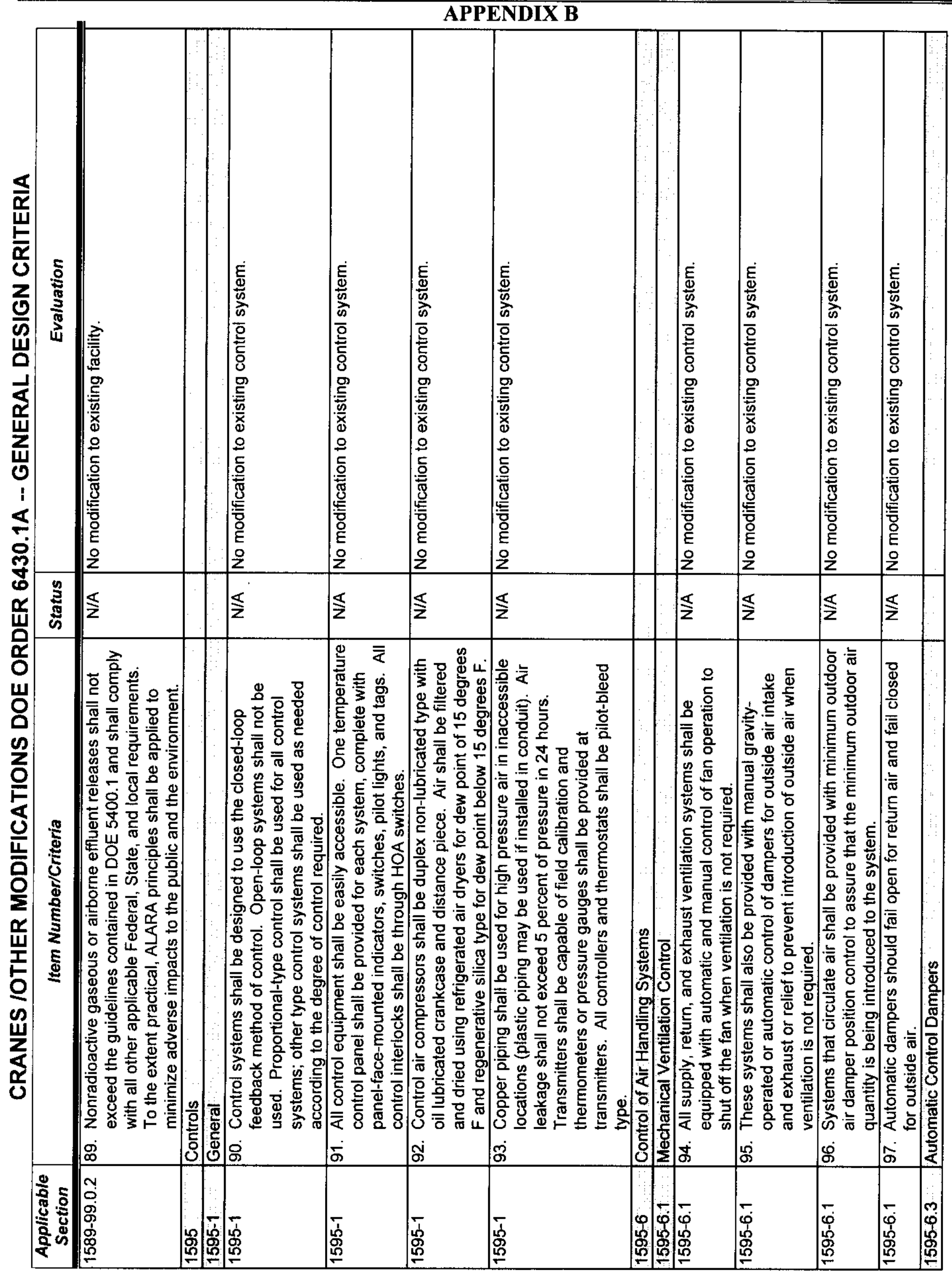


SNF-4775, Rev. 2

Project A.5/A.6

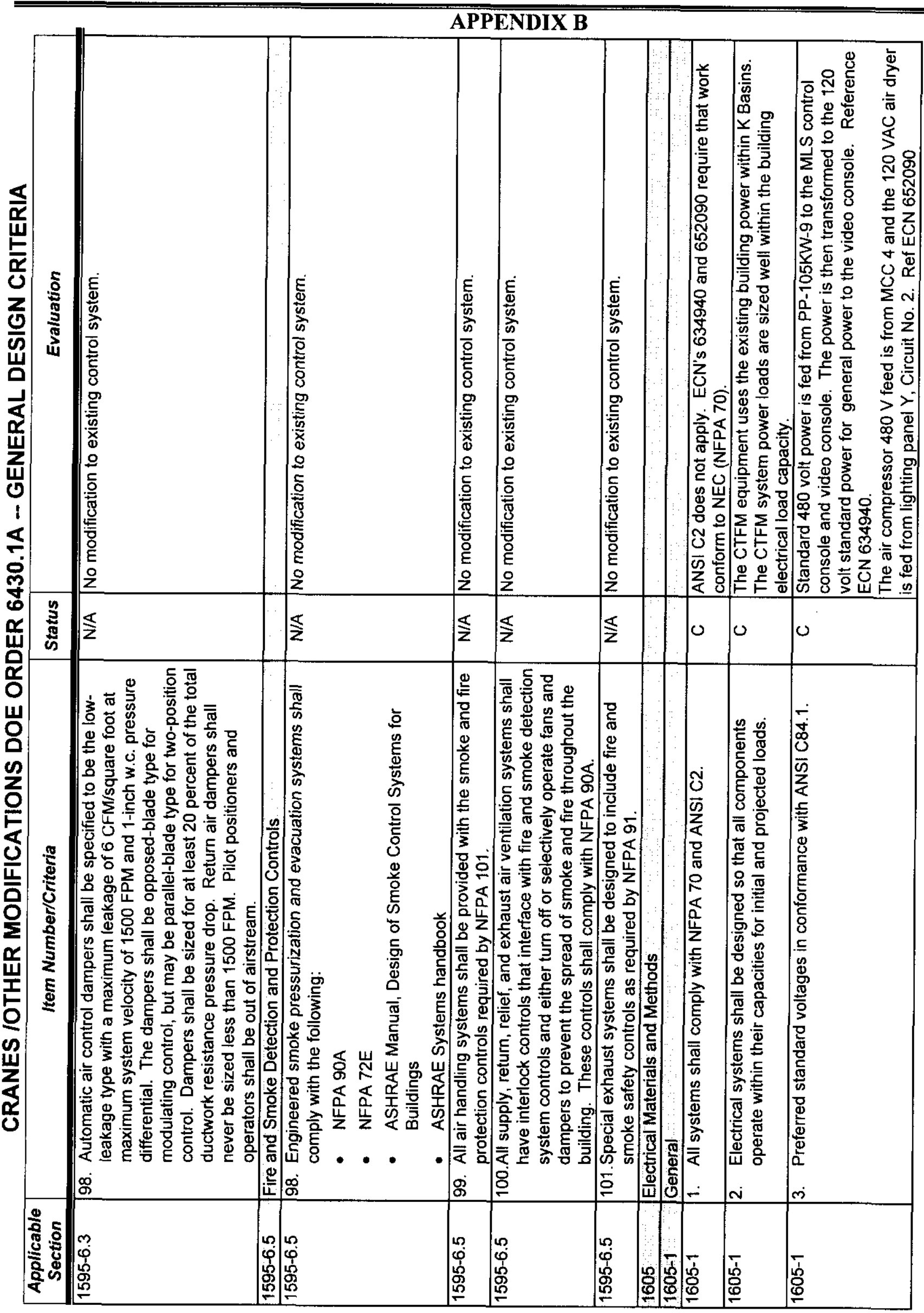

Page B-88 
SNF-4775, Rev. 2

Project A.5/A.6

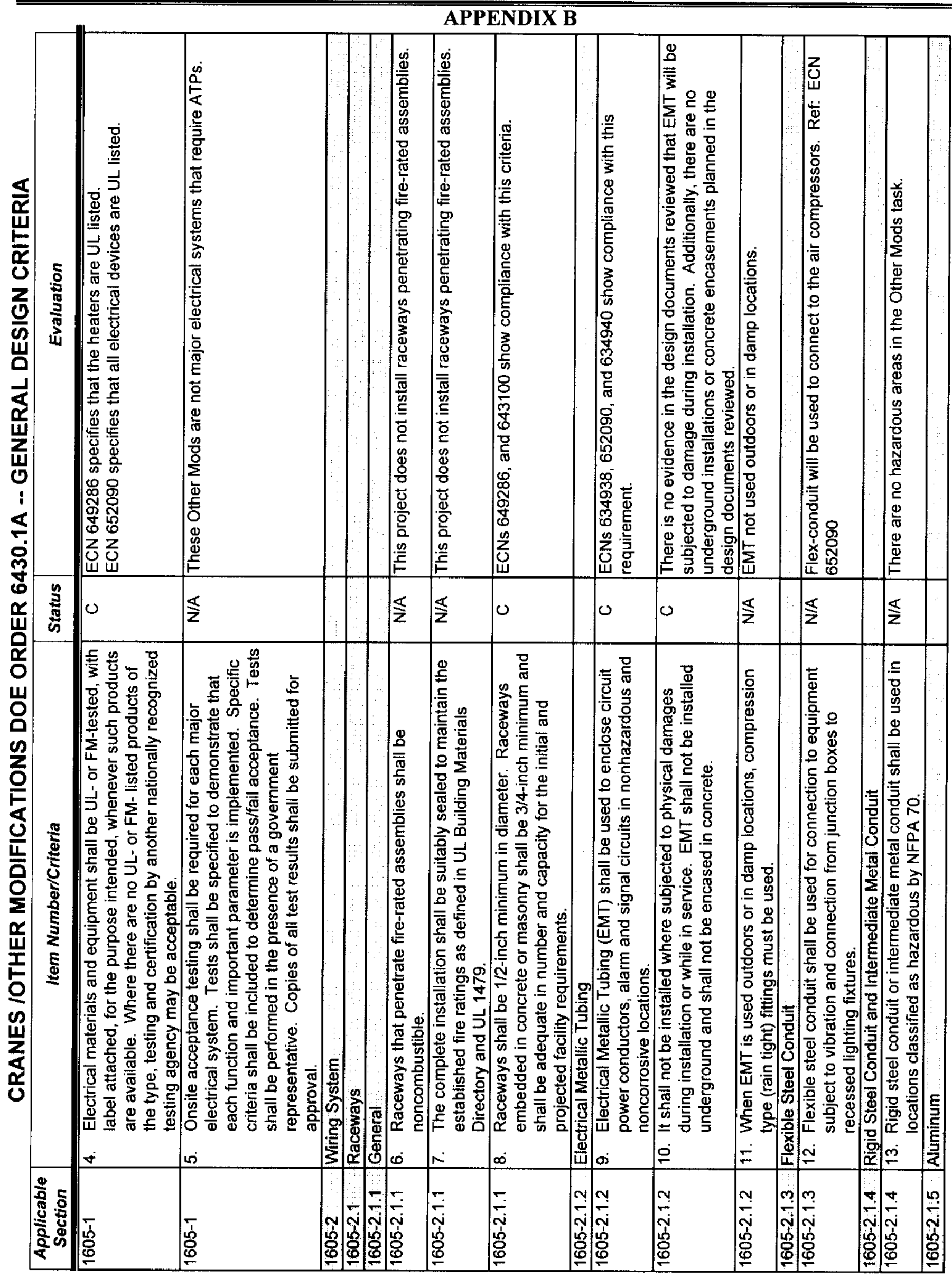




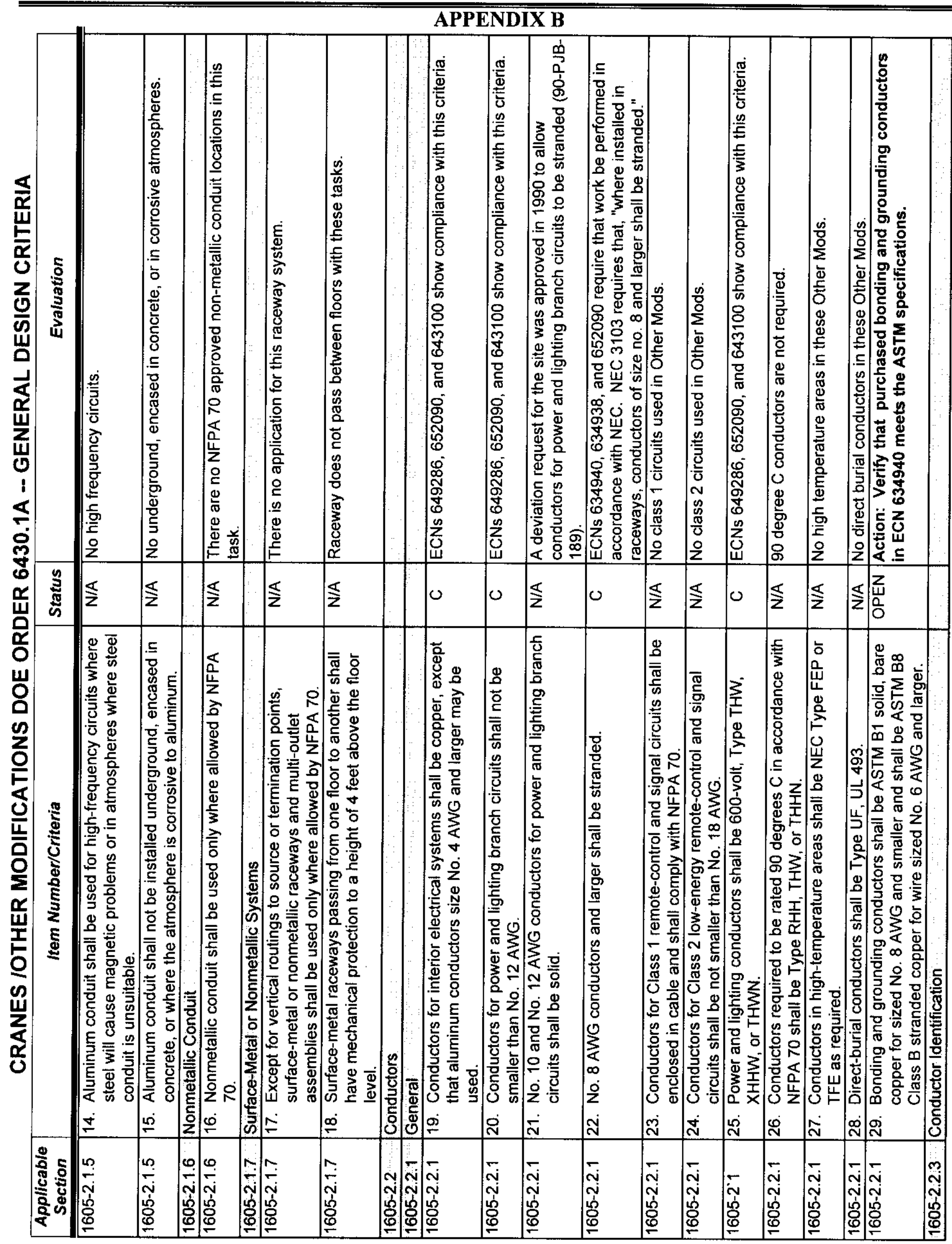




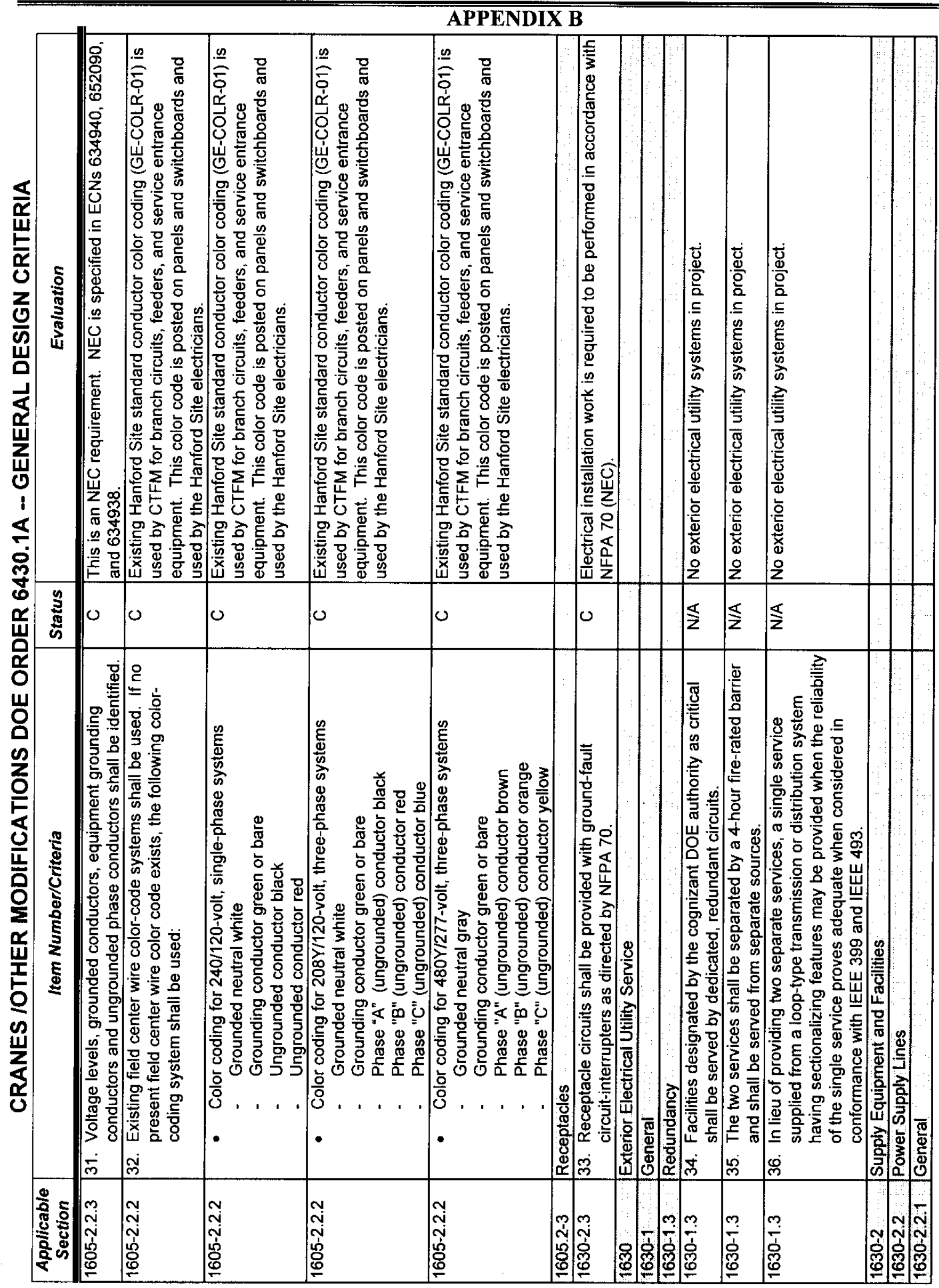




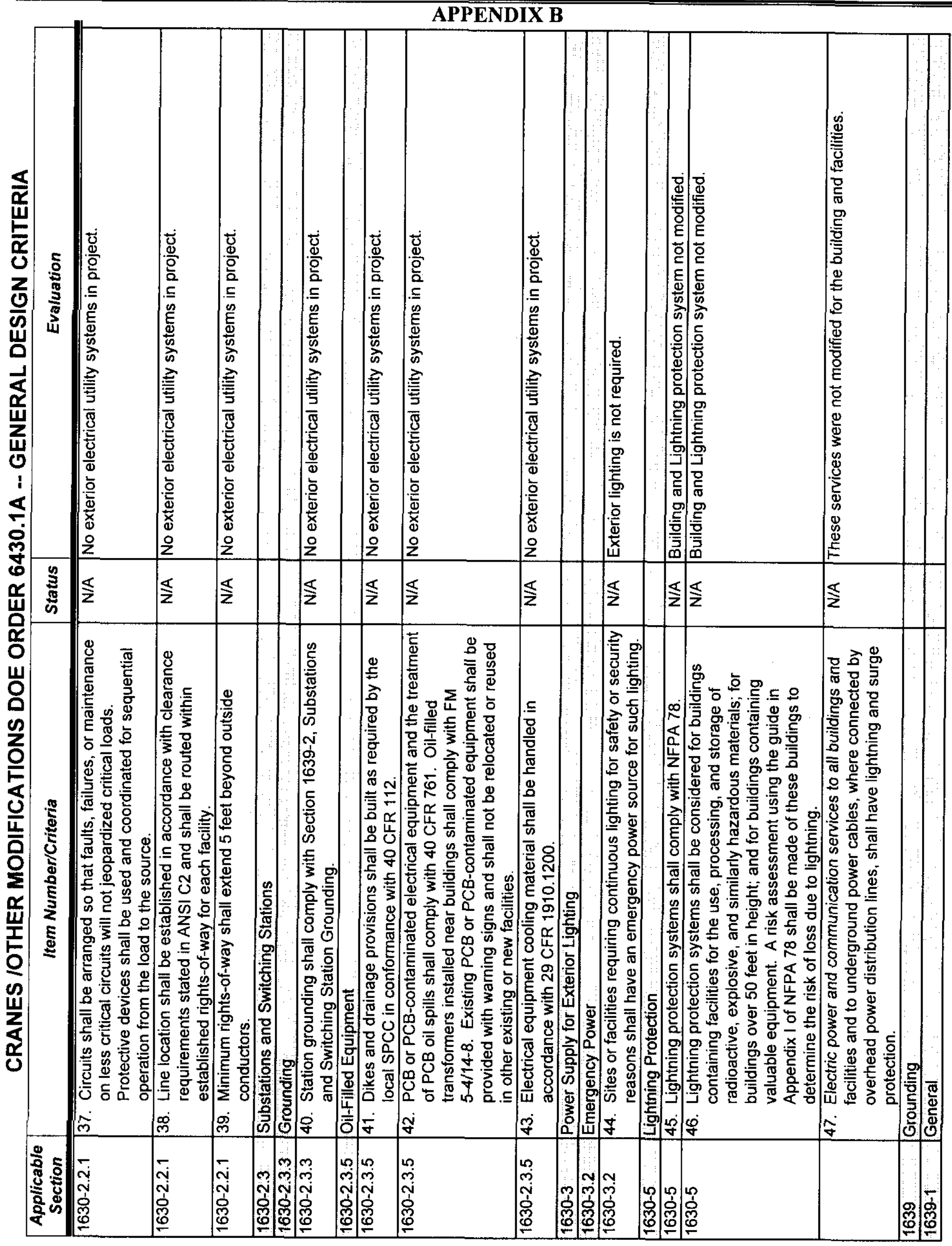


SNF-4775, Rev. 2

Project A.5/A.6

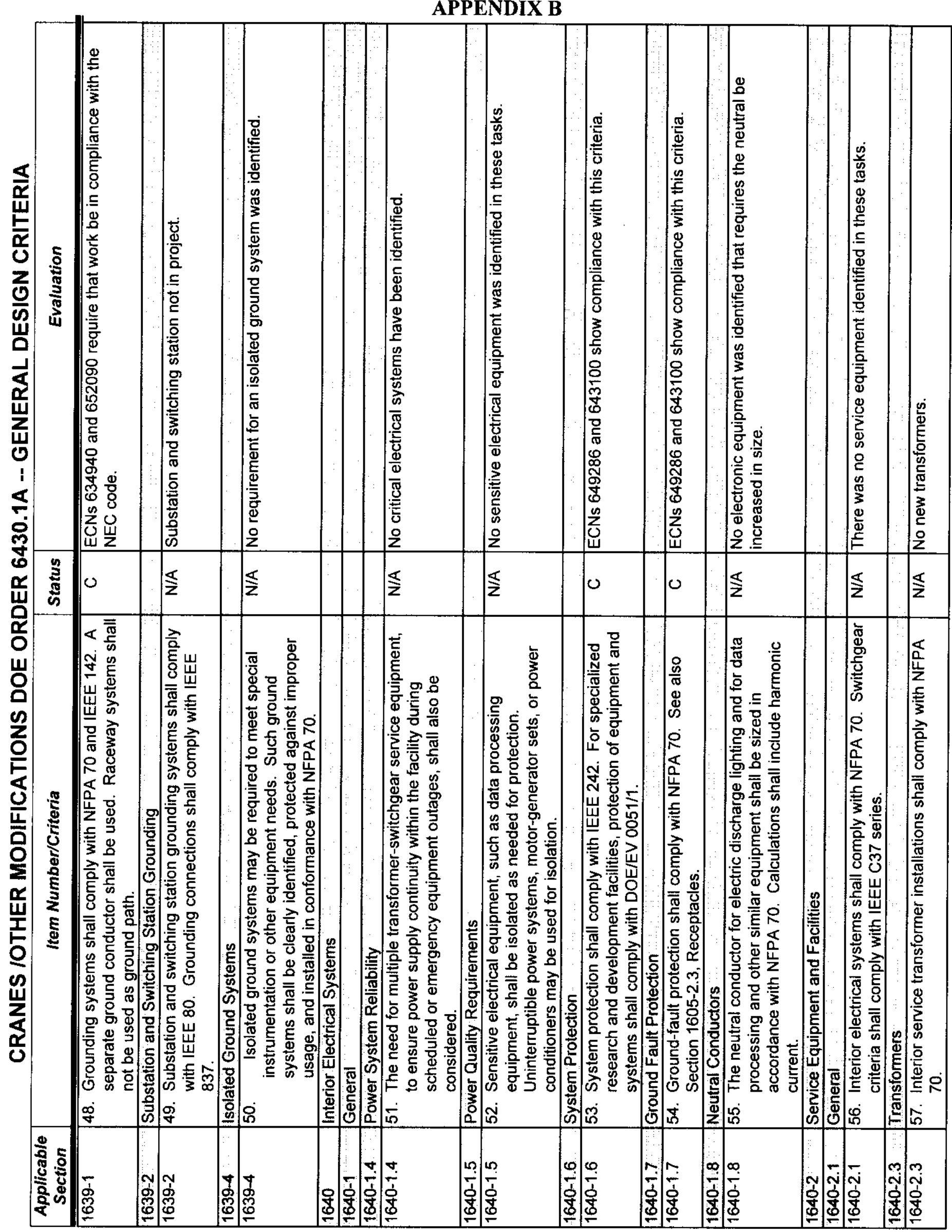




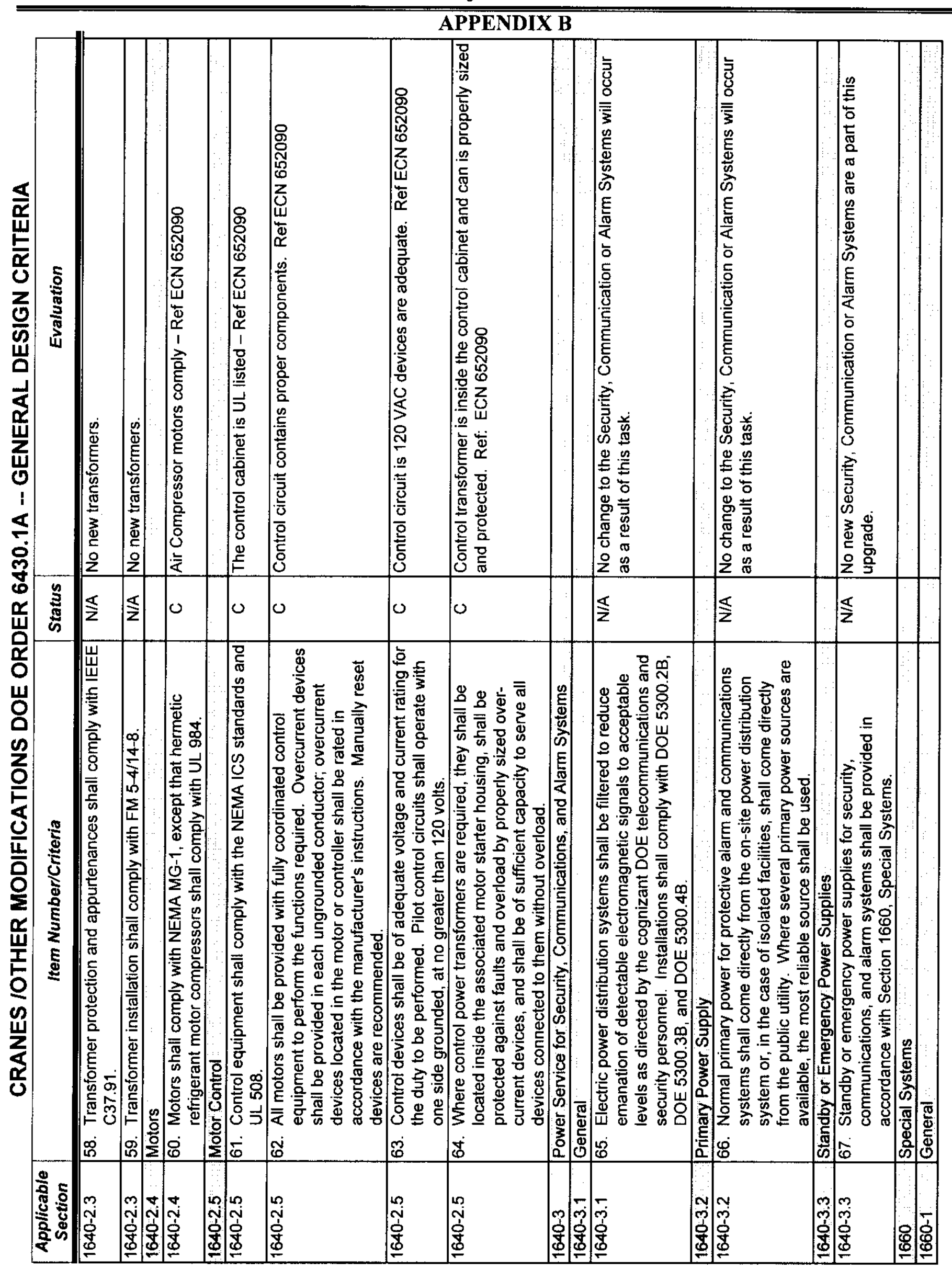




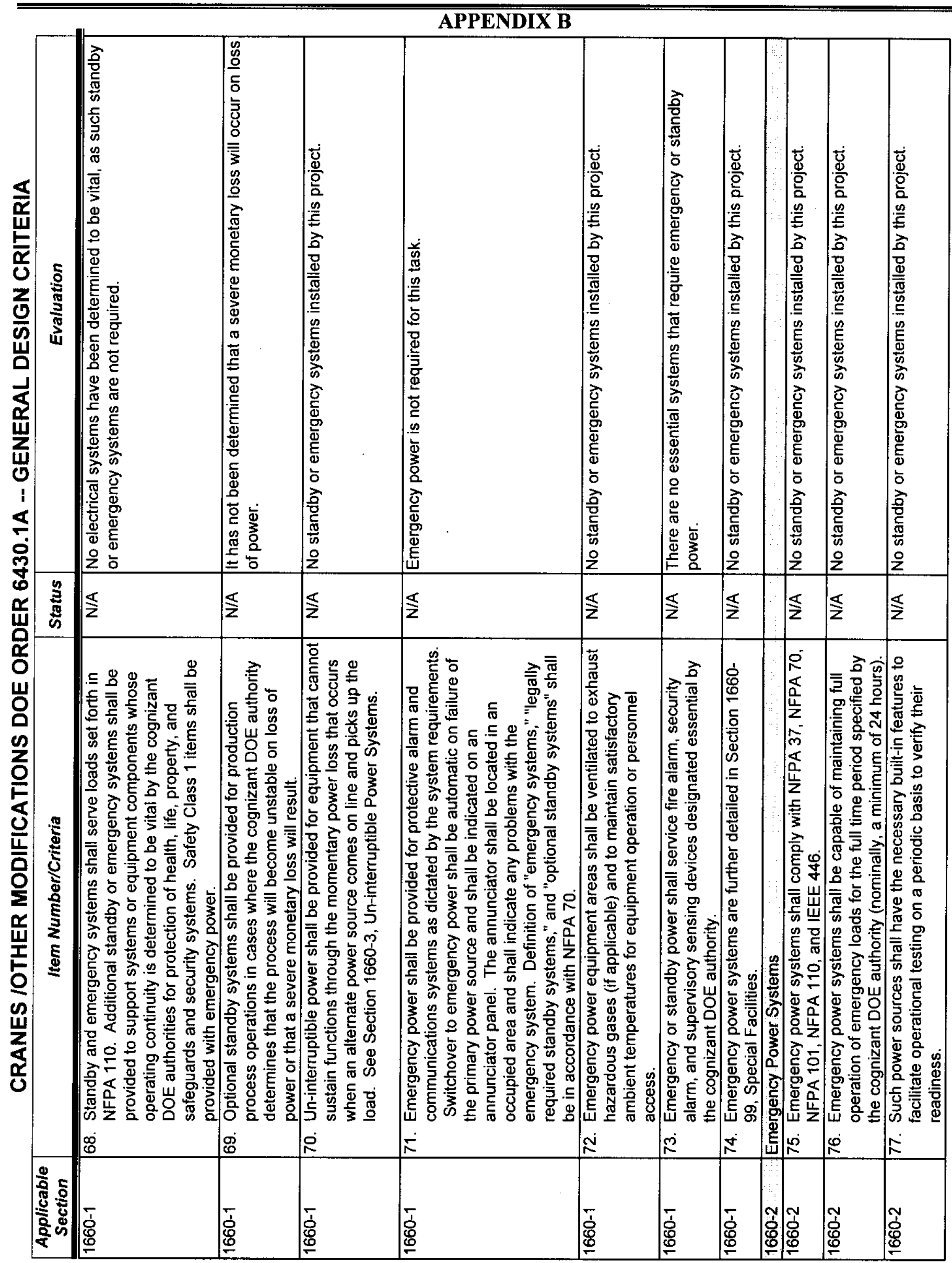




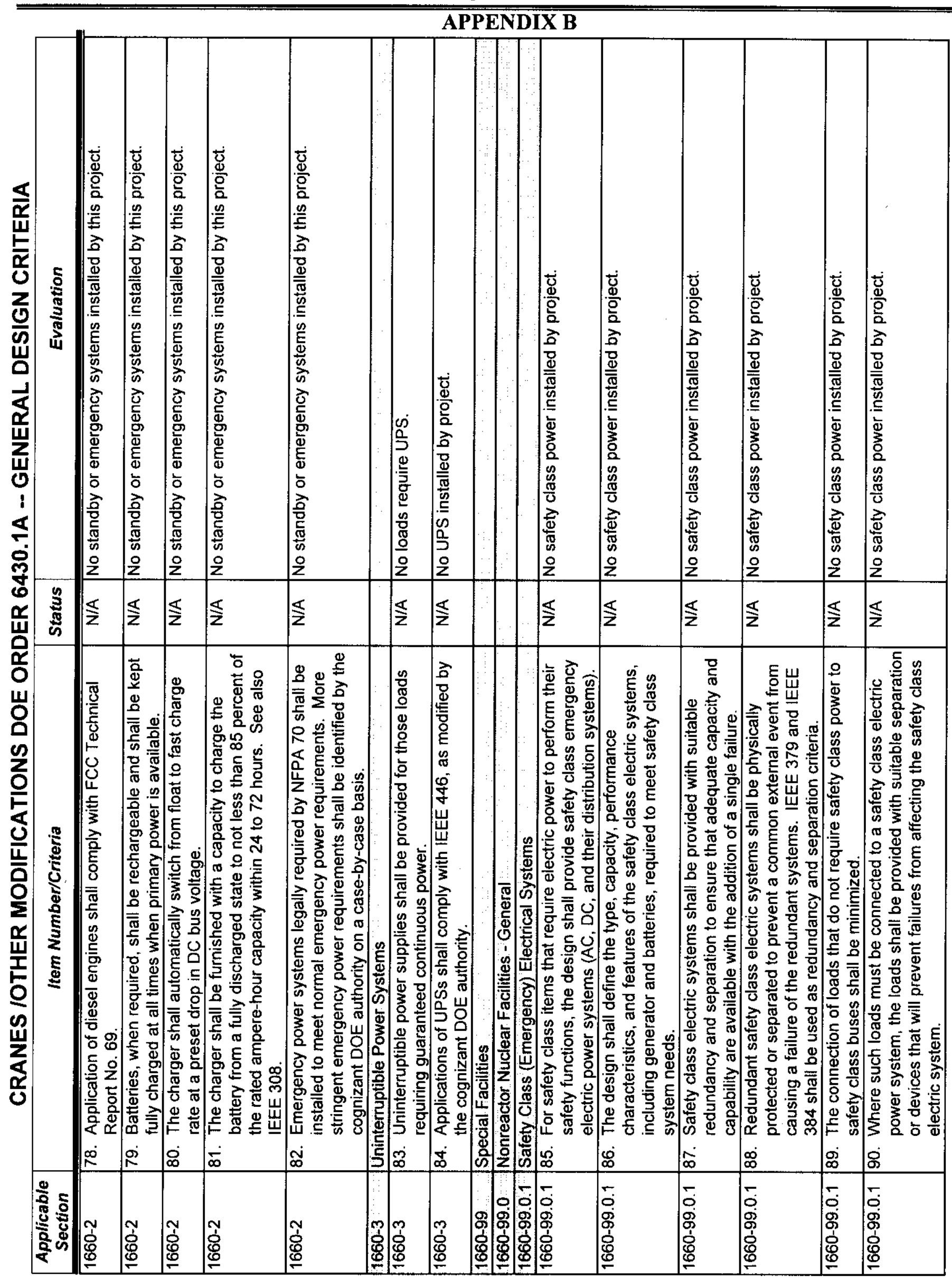


SNF-4775, Rev. 2

Project A.5/A.6

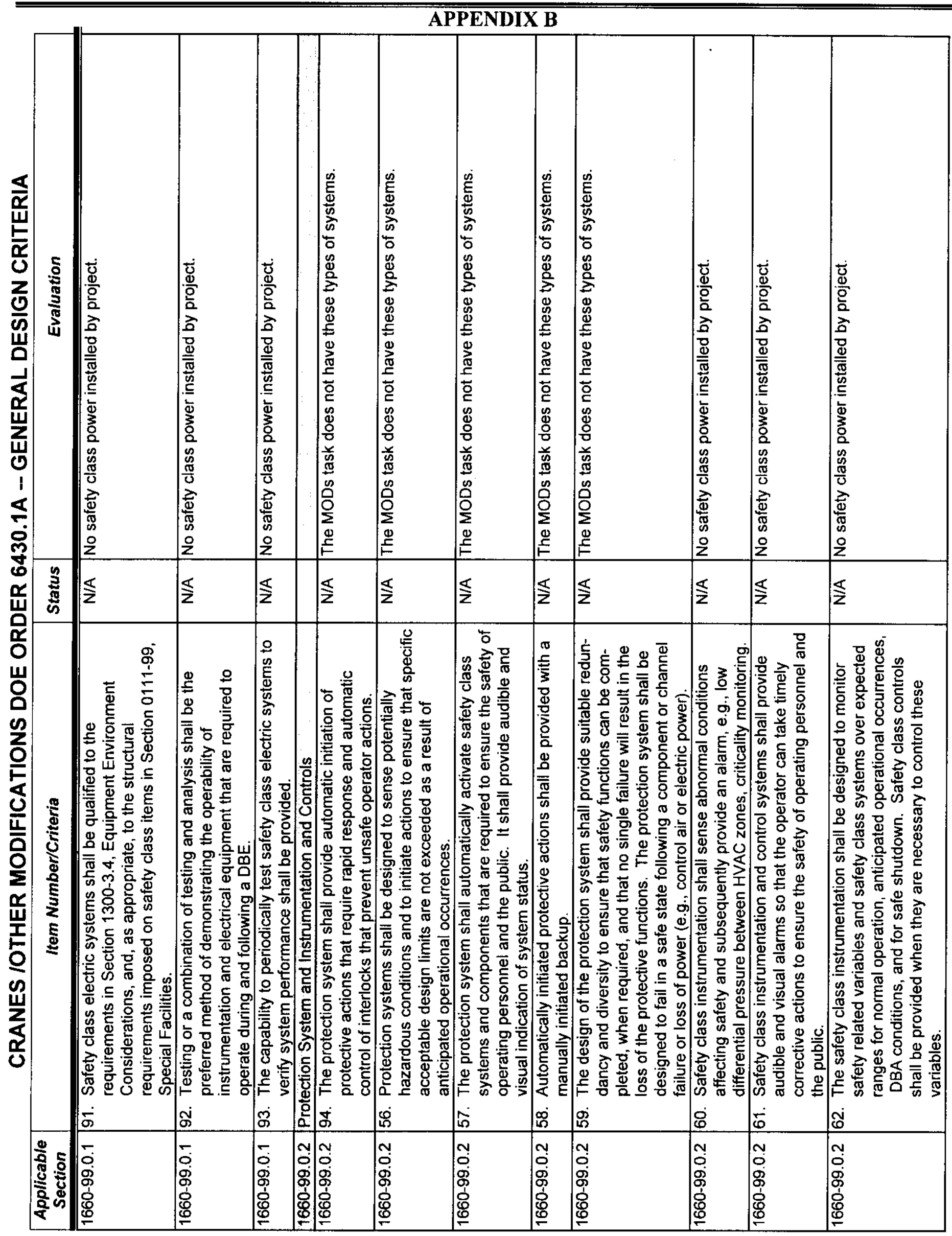


SNF-4775, Rev. 2

Project A.5/A.6

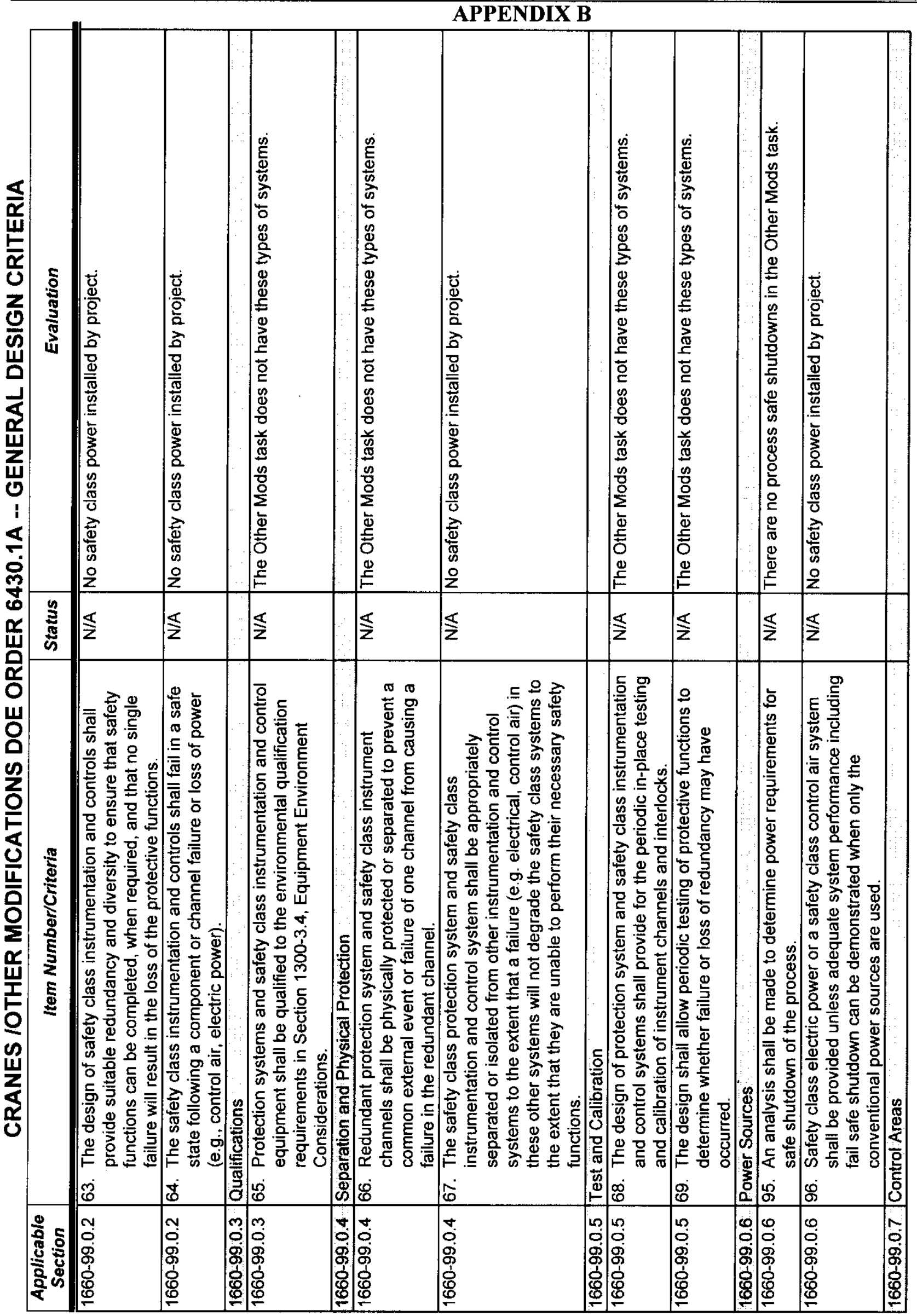




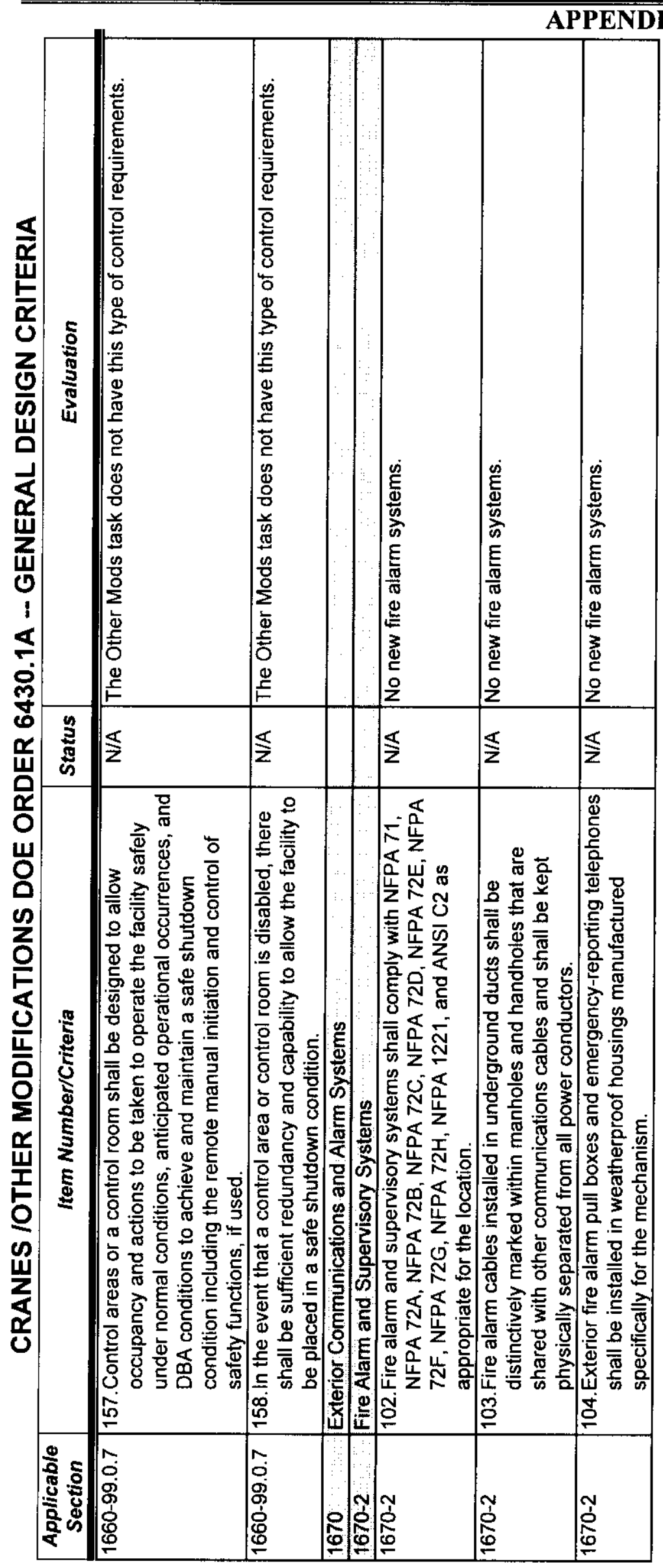

Page B-99 
SNF-4775, Rev. 2

Project A.5/A.6

APPENDIX C

CASK LOADOUT SYSTEM (CLS) TABLE

DOE ORDER 6430.1A - GENERAL DESIGN CRITERIA

OPEN ITEMS TRACKING

CONSISTING OF 16 PAGES

INCLUDING COVERSHEET

Page C-1 
SSNF-4775, Rev. 2

Project A.5/A.6

APPENDIX C

는

\begin{tabular}{|c|c|c|c|c|}
\hline 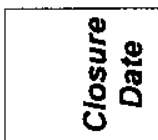 & (1) & & $\frac{8}{\frac{8}{n}}$ & \\
\hline 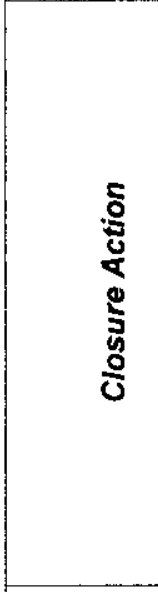 & (1) & 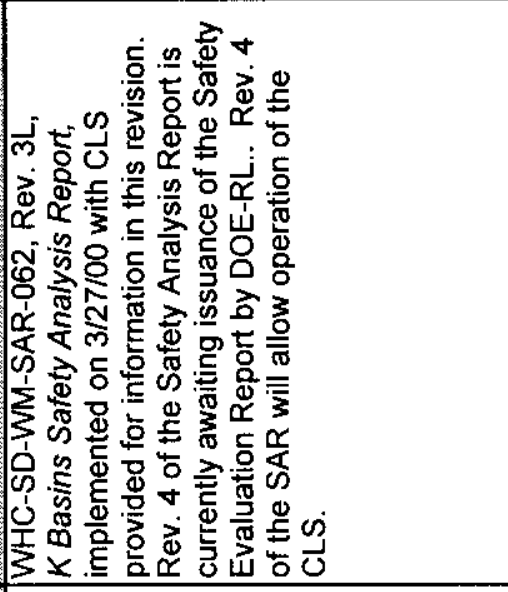 & 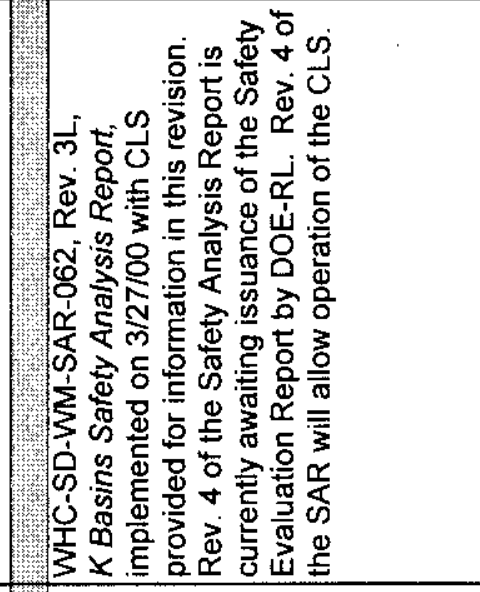 & \\
\hline 今ัّ & & 昰 & ไั: & \\
\hline 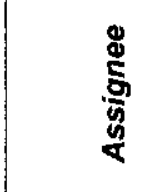 & & 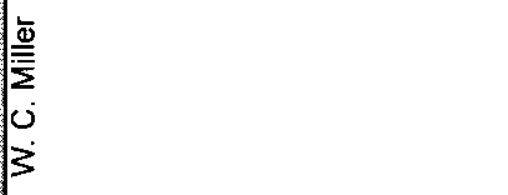 & $\sqrt{\frac{0}{2}}$ & \\
\hline 宽 & 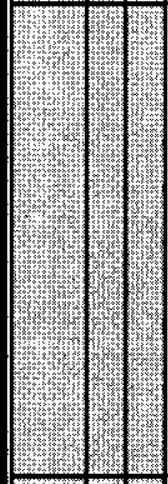 & 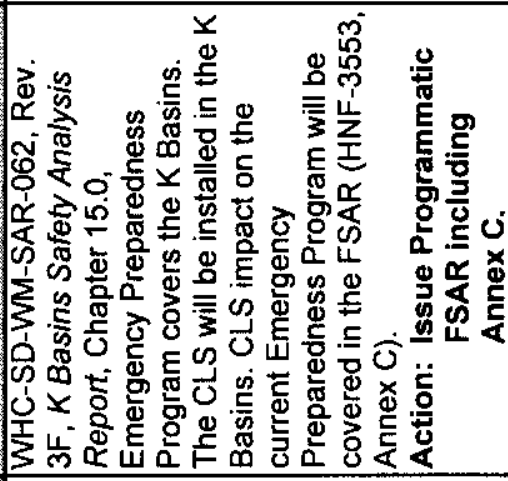 & 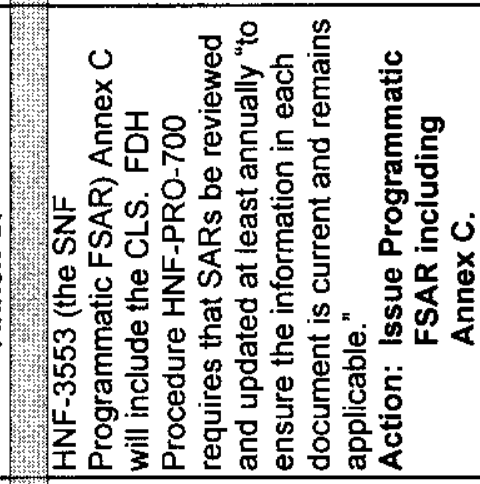 & \\
\hline$\Xi$ & & $\begin{array}{l} \\
\mathbb{J} \\
0 \\
0 \\
0 \\
\end{array}$ & 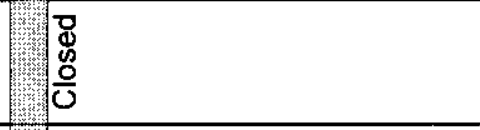 & \\
\hline 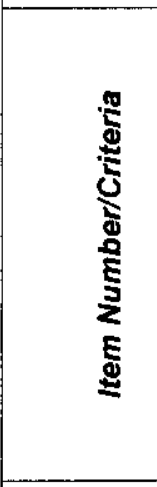 & 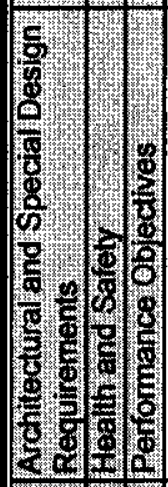 & 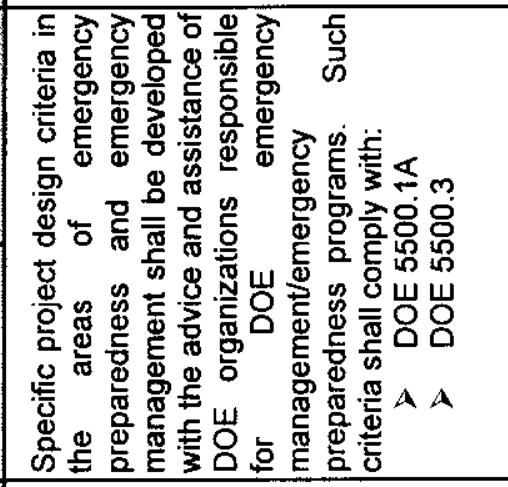 & 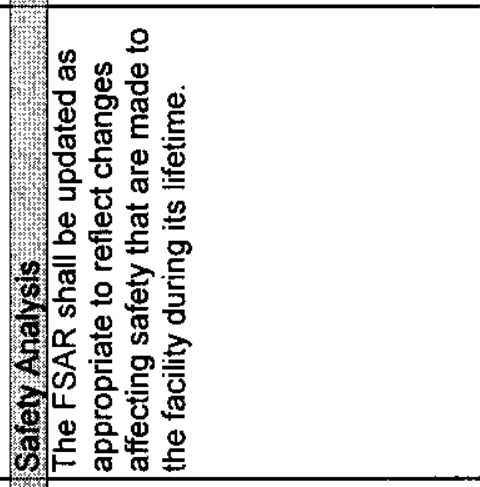 & 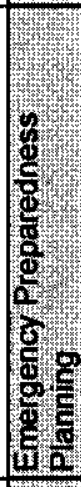 \\
\hline 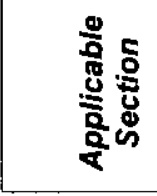 & 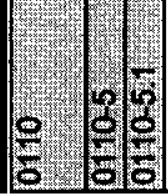 & $\frac{1}{60}$ & 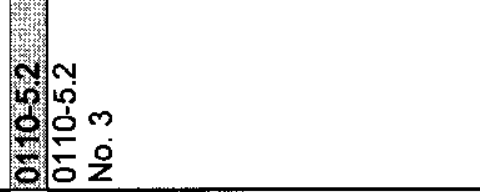 & $\frac{3}{3}$ \\
\hline
\end{tabular}

Page C-2 
SSNF-4775, Rev. 2

Project A.5/A.6

APPENDIX C

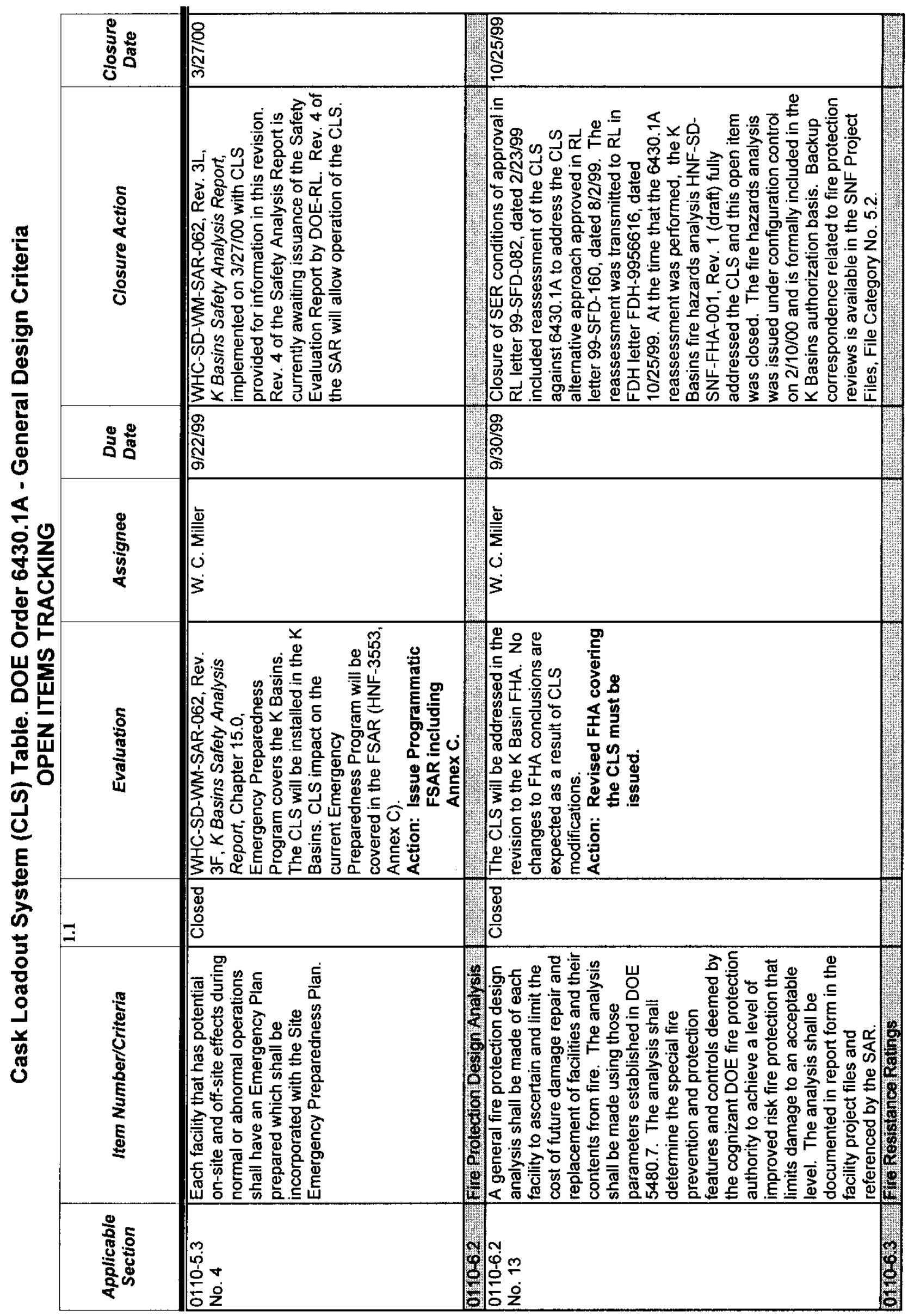

Page C-3 
SSNF-4775, Rev. 2

Project A.5/A.6

APPENDIX C

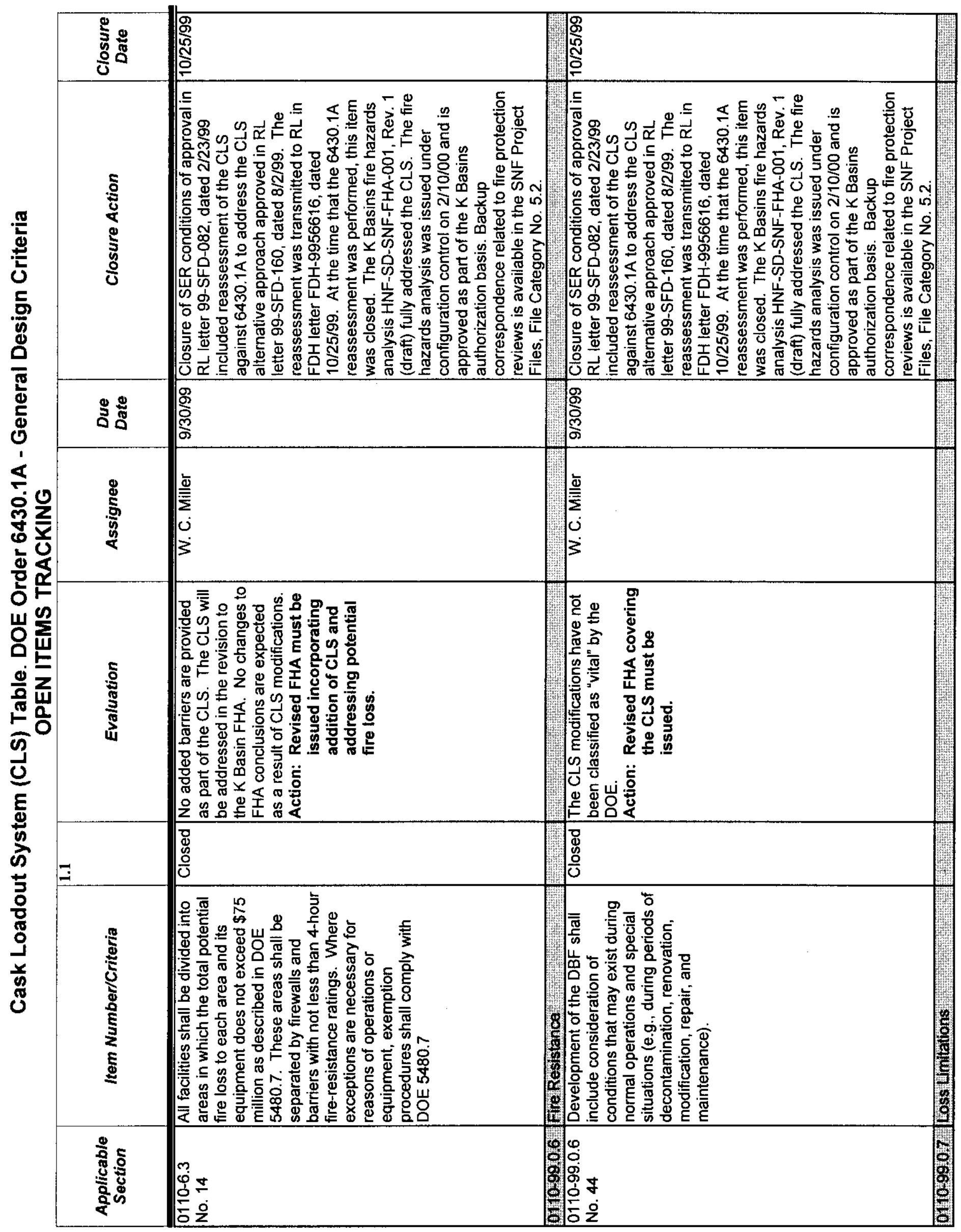

Page C-4 
SSNF-4775, Rev. 2

Project A.5/A.6

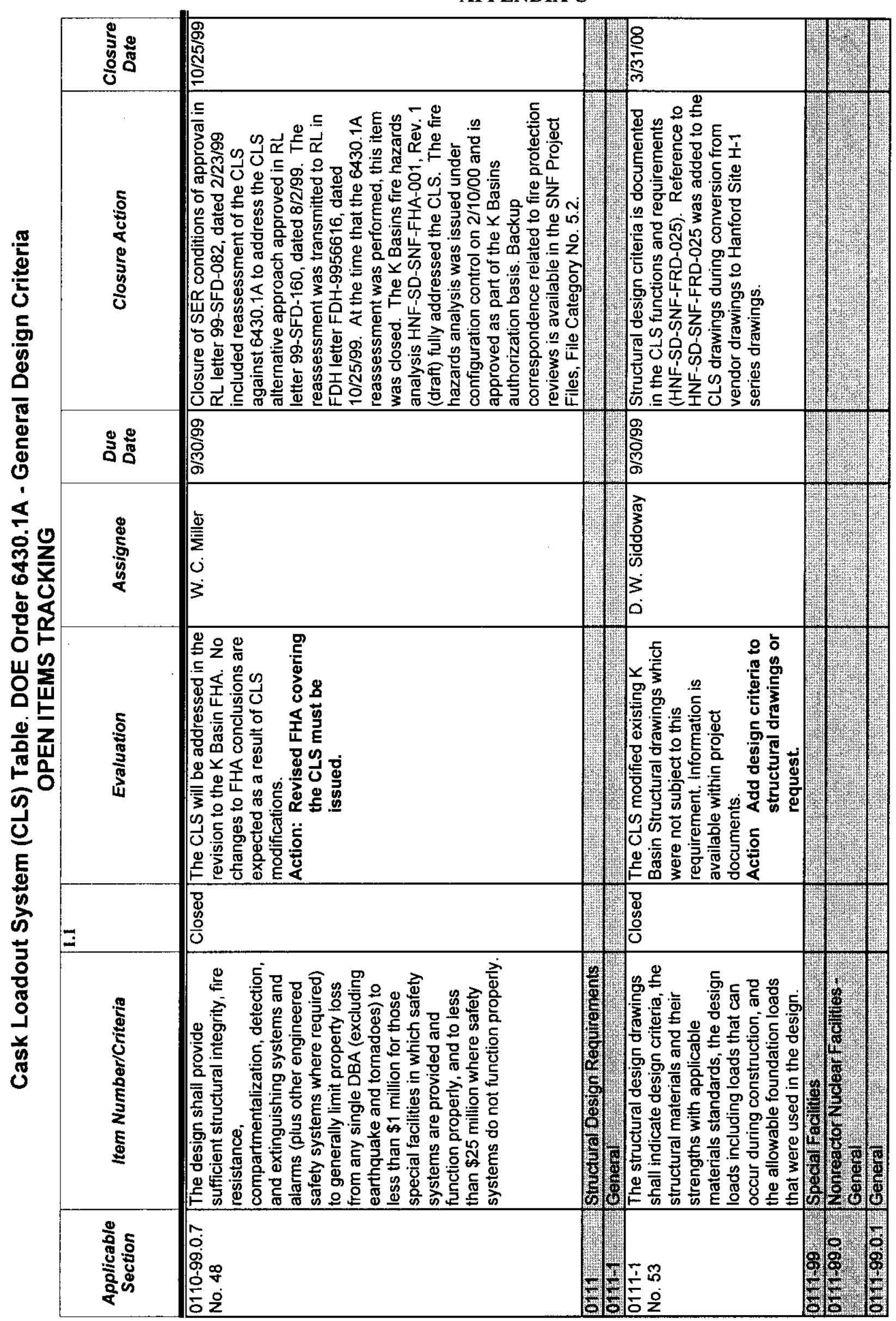


SSNF-4775, Rev. 2

Project A.5/A.6

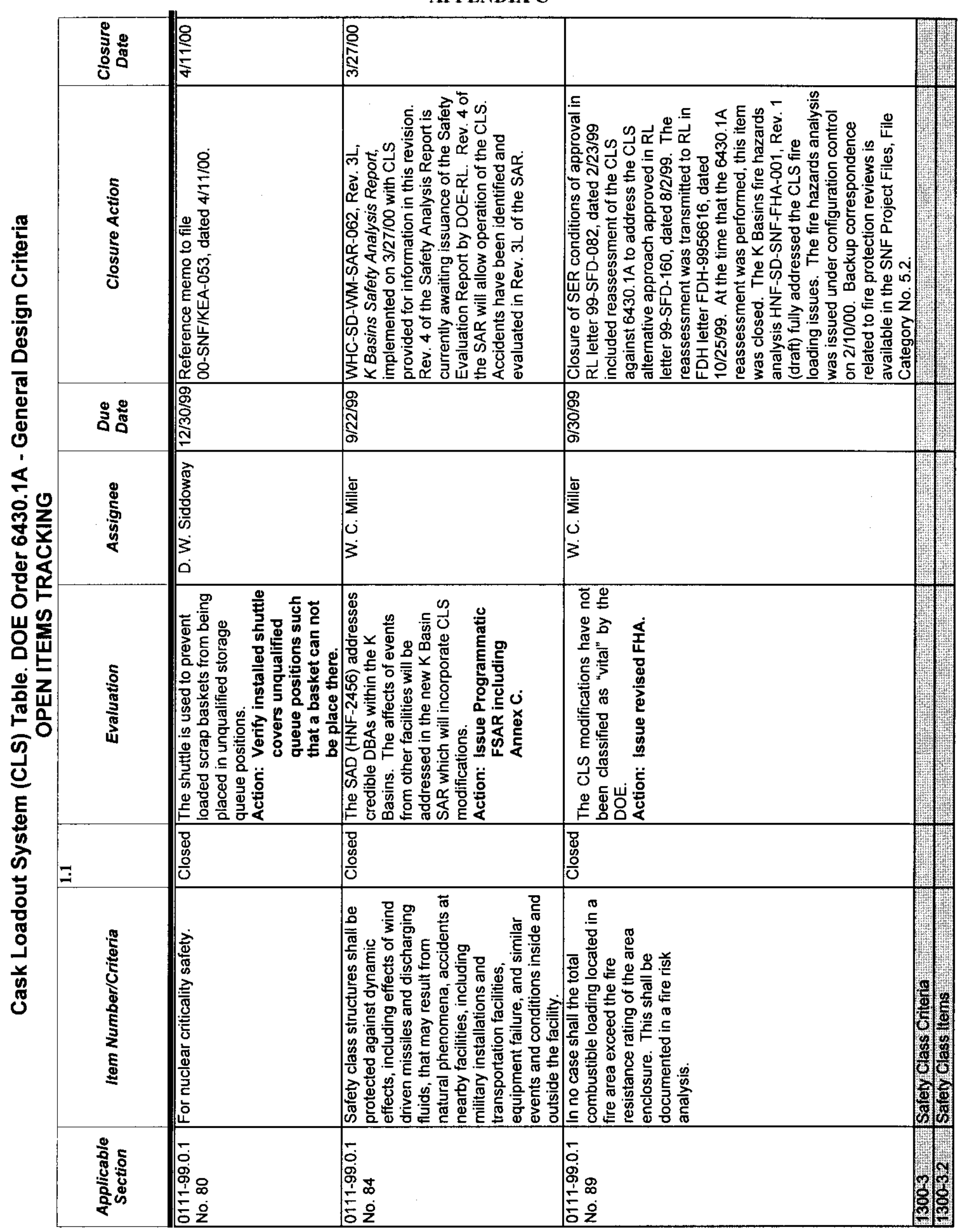

Page C-6 
SSNF-4775, Rev. 2

Project A.5/A.6

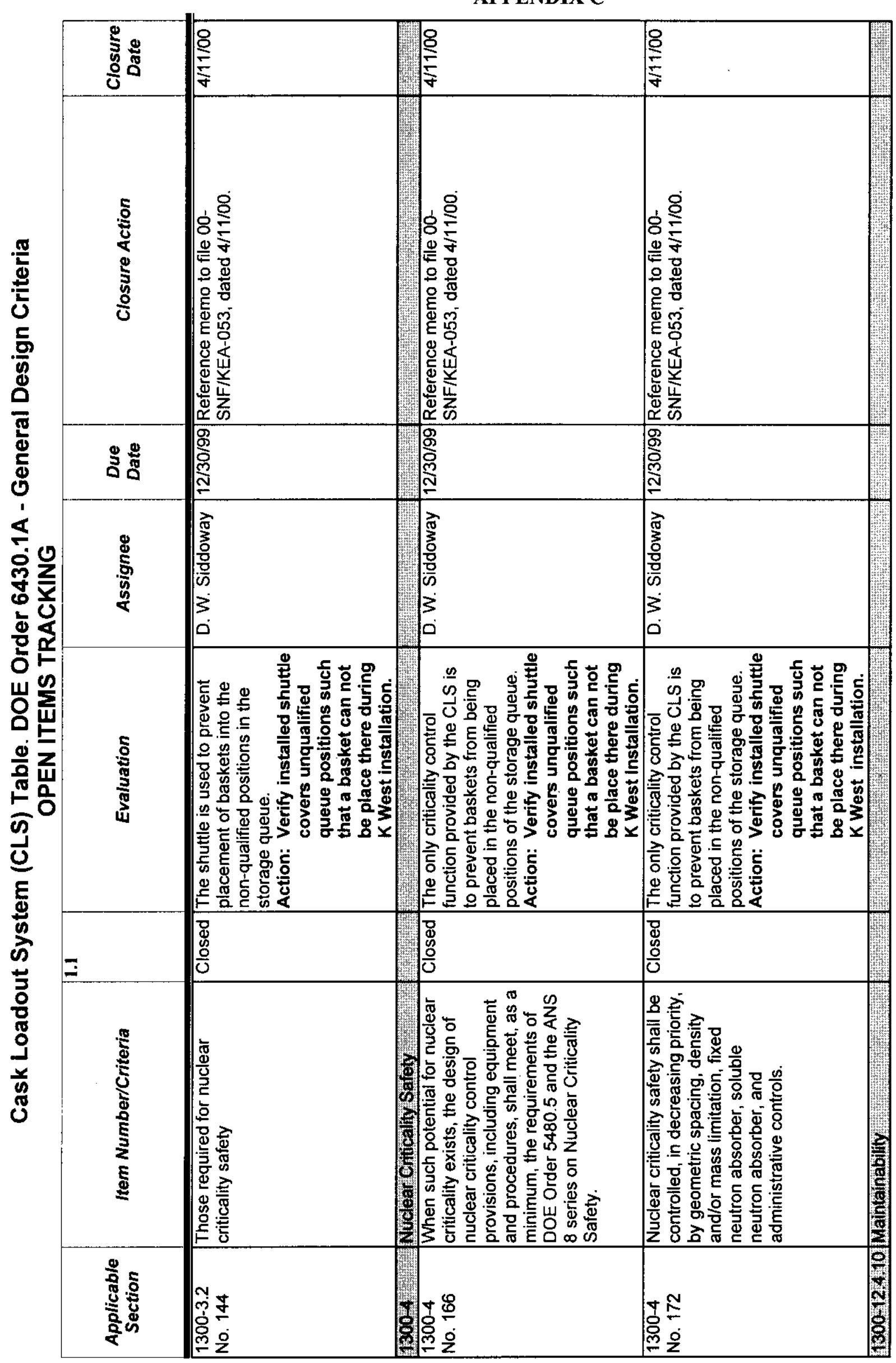

Page C-7 
SSNF-4775, Rev. 2

Project A.5/A.6

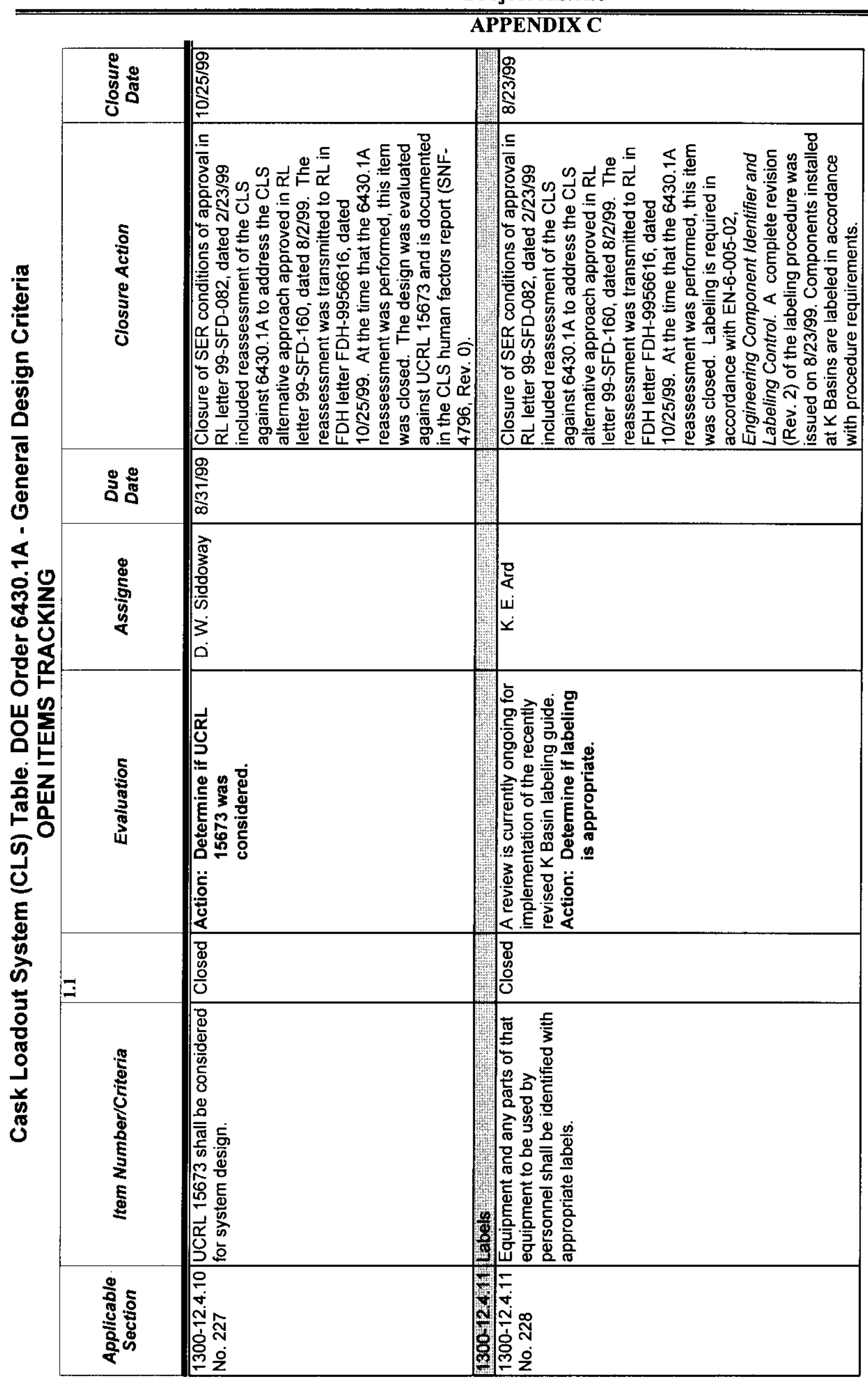

Page C-8 
SSNF-4775, Rev. 2

Project A.5/A.6

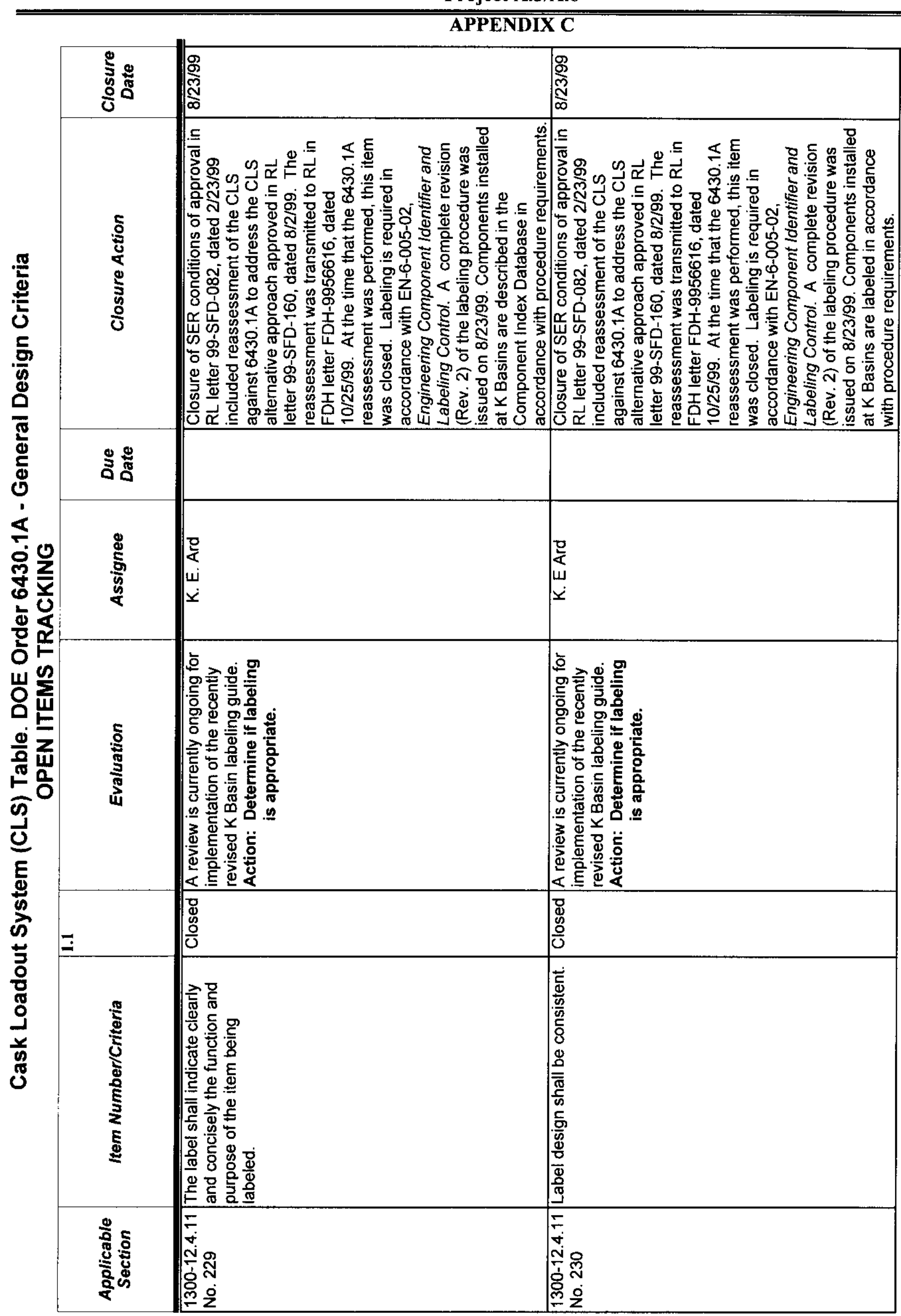

Page C-9 
SSNF-4775, Rev. 2

Project A.5/A.6

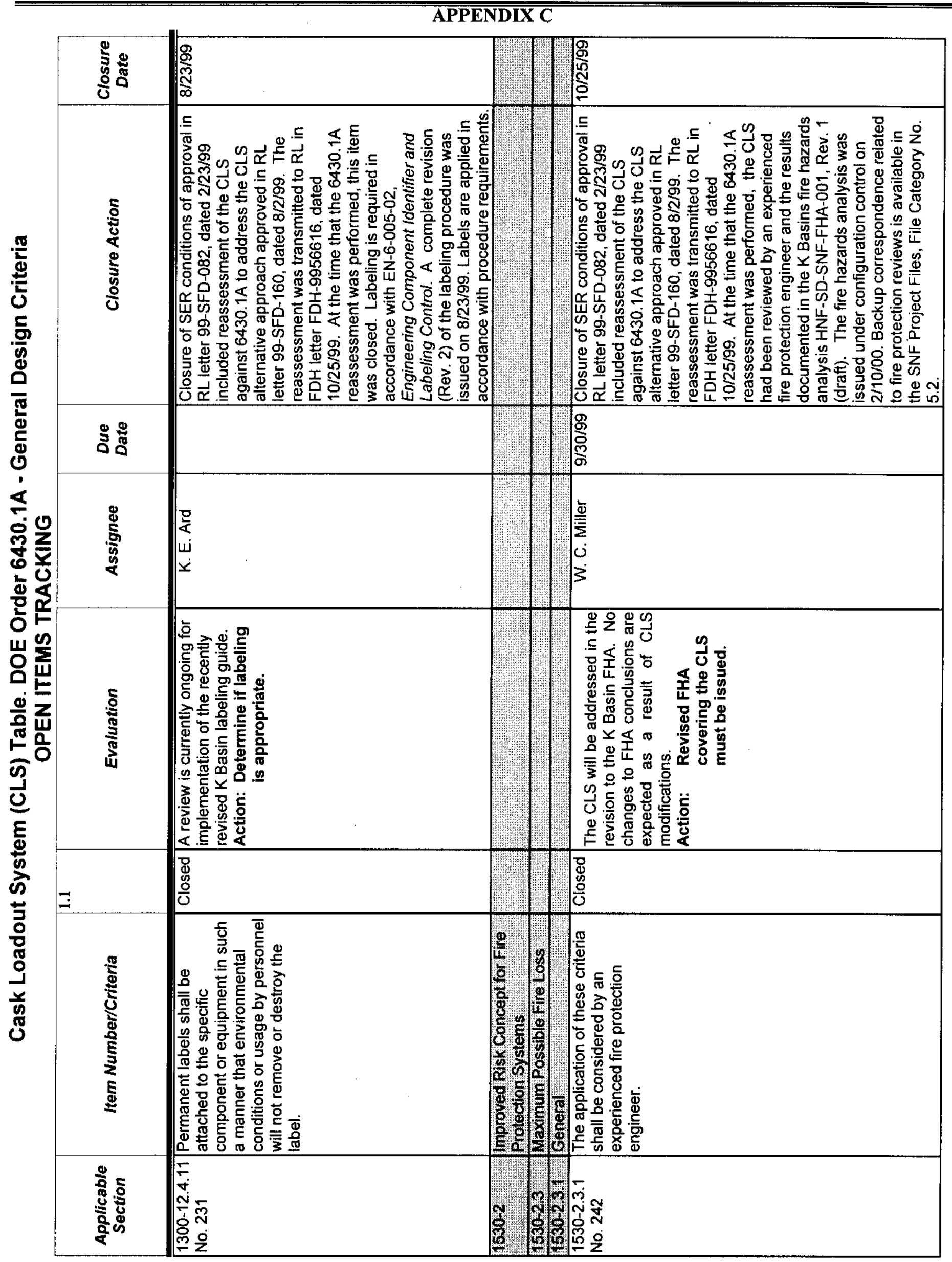


SSNF-4775, Rev. 2

Project A.5/A.6

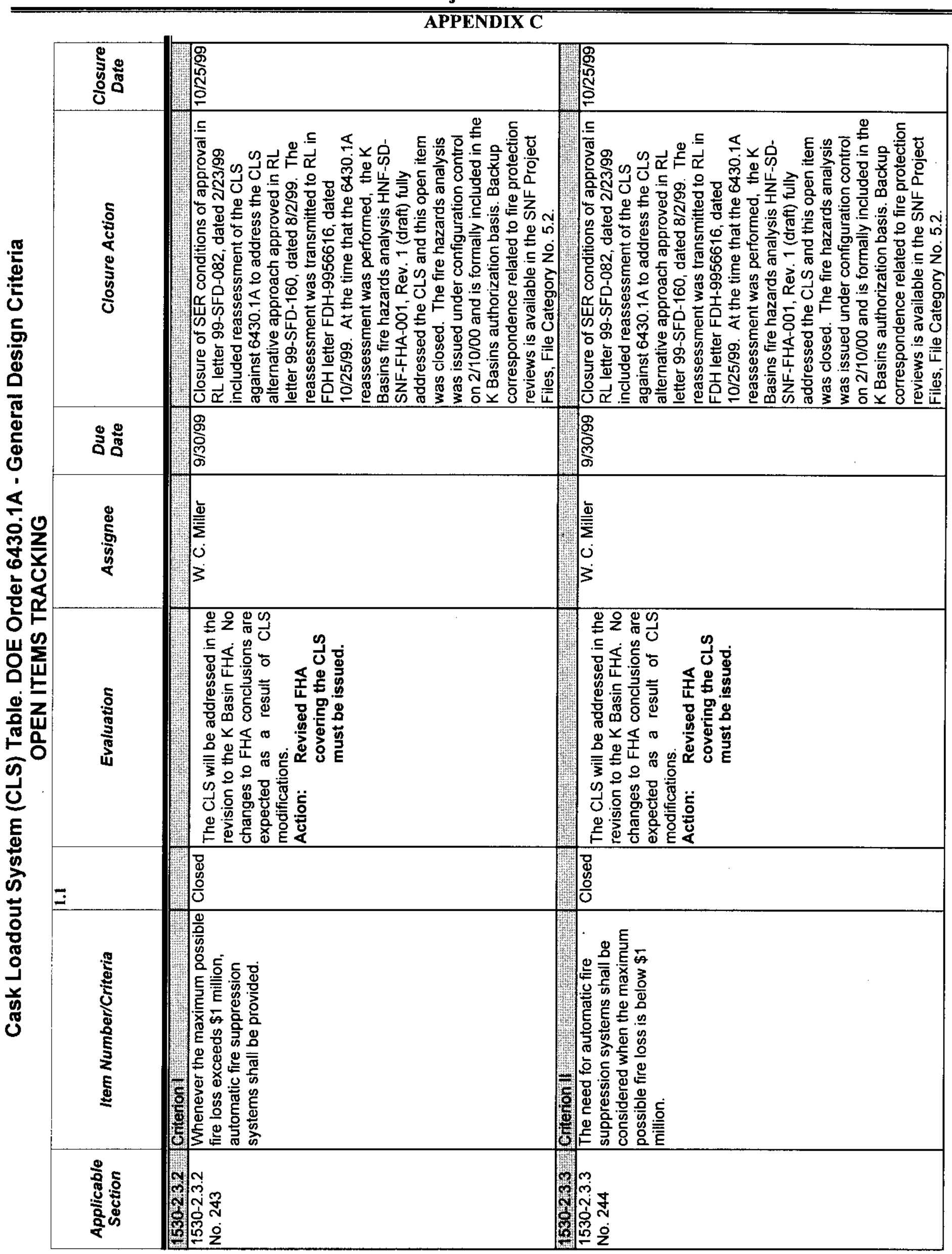

Page C-11 
SSNF-4775, Rev. 2

Project A.5/A.6 
SSNF-4775, Rev. 2

Project A.5/A.6

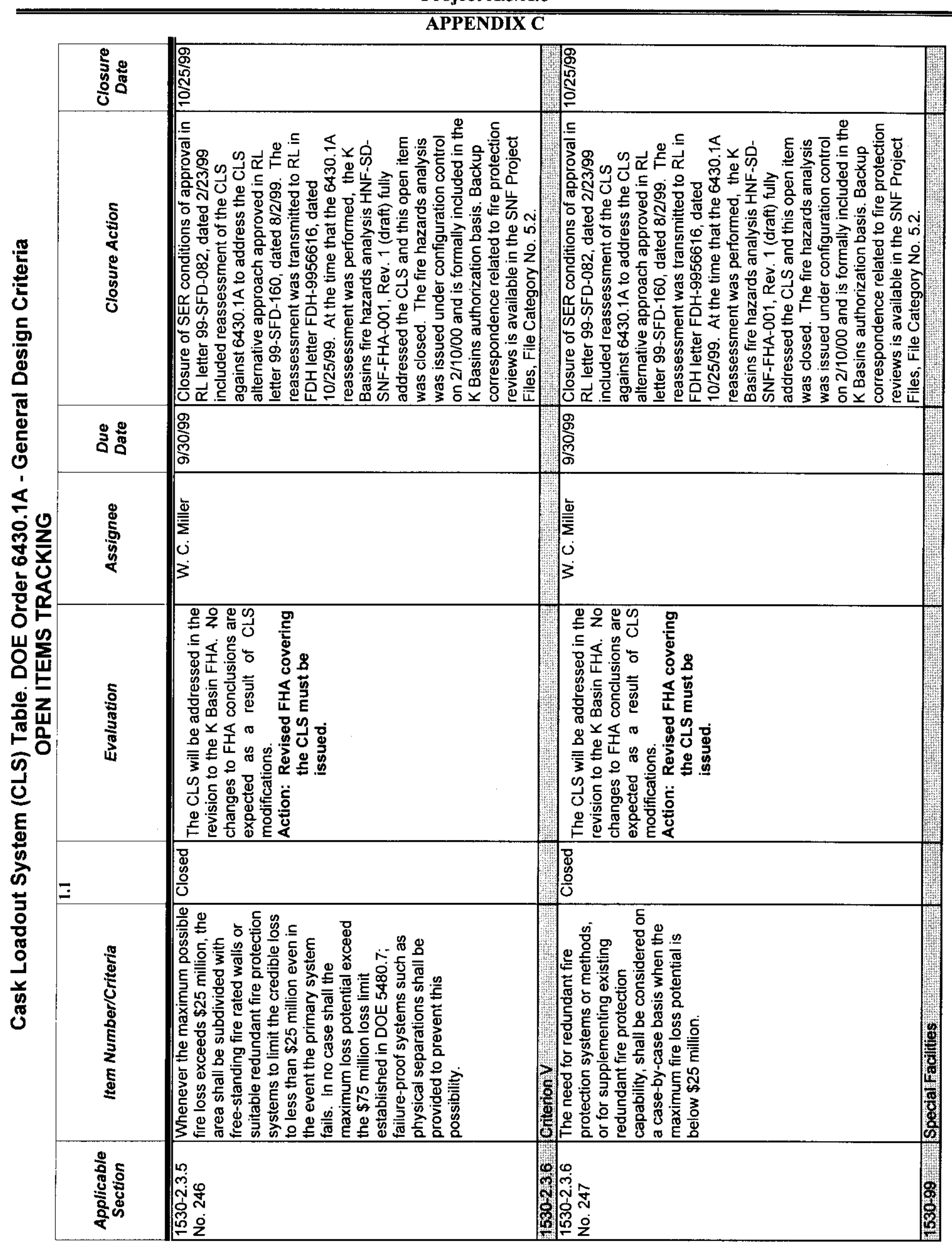

Page C-13 
SSNF-4775, Rev. 2

Project A.5/A.6

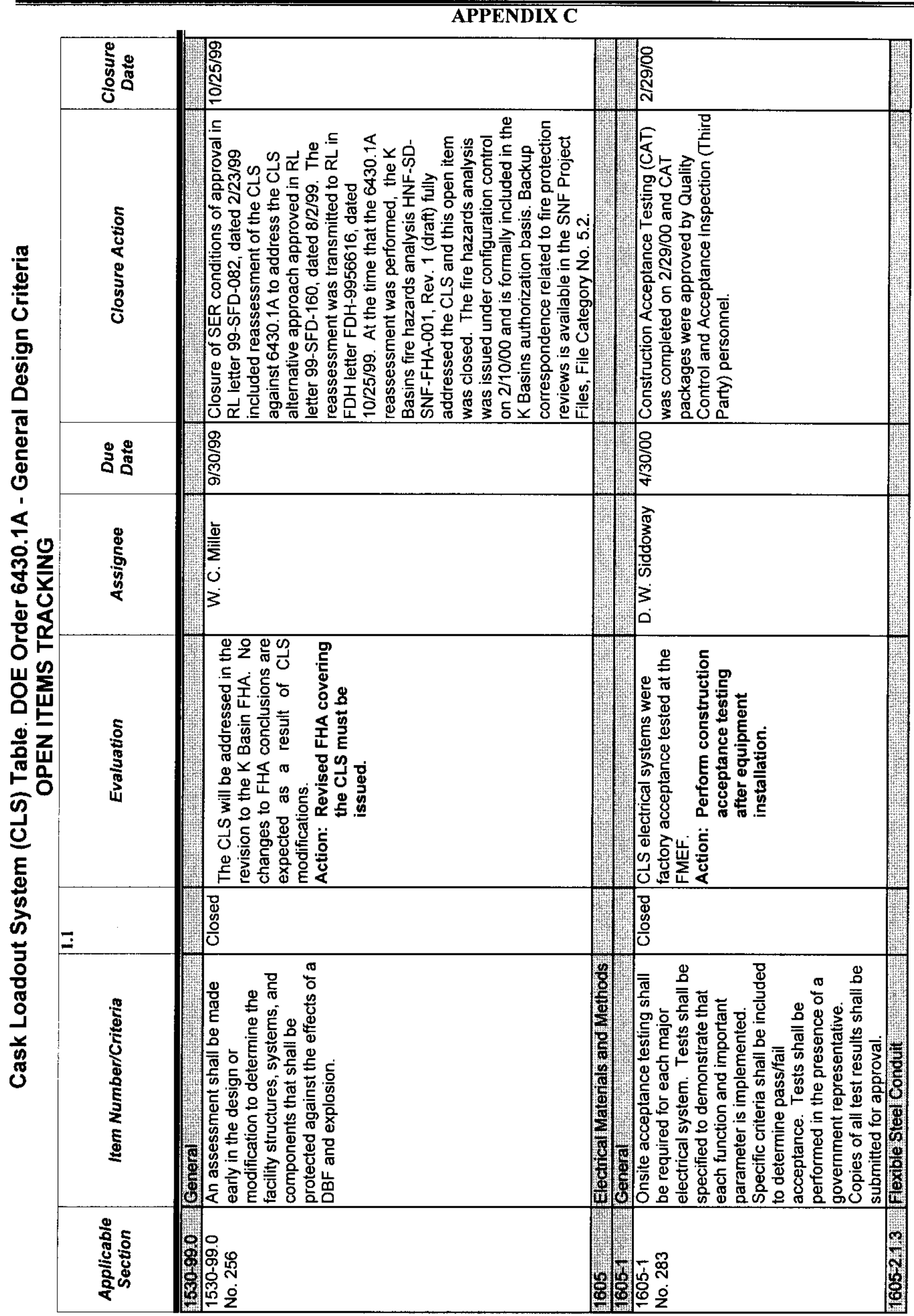

Page C-14 
SSNF-4775, Rev. 2

Project A.5/A.6

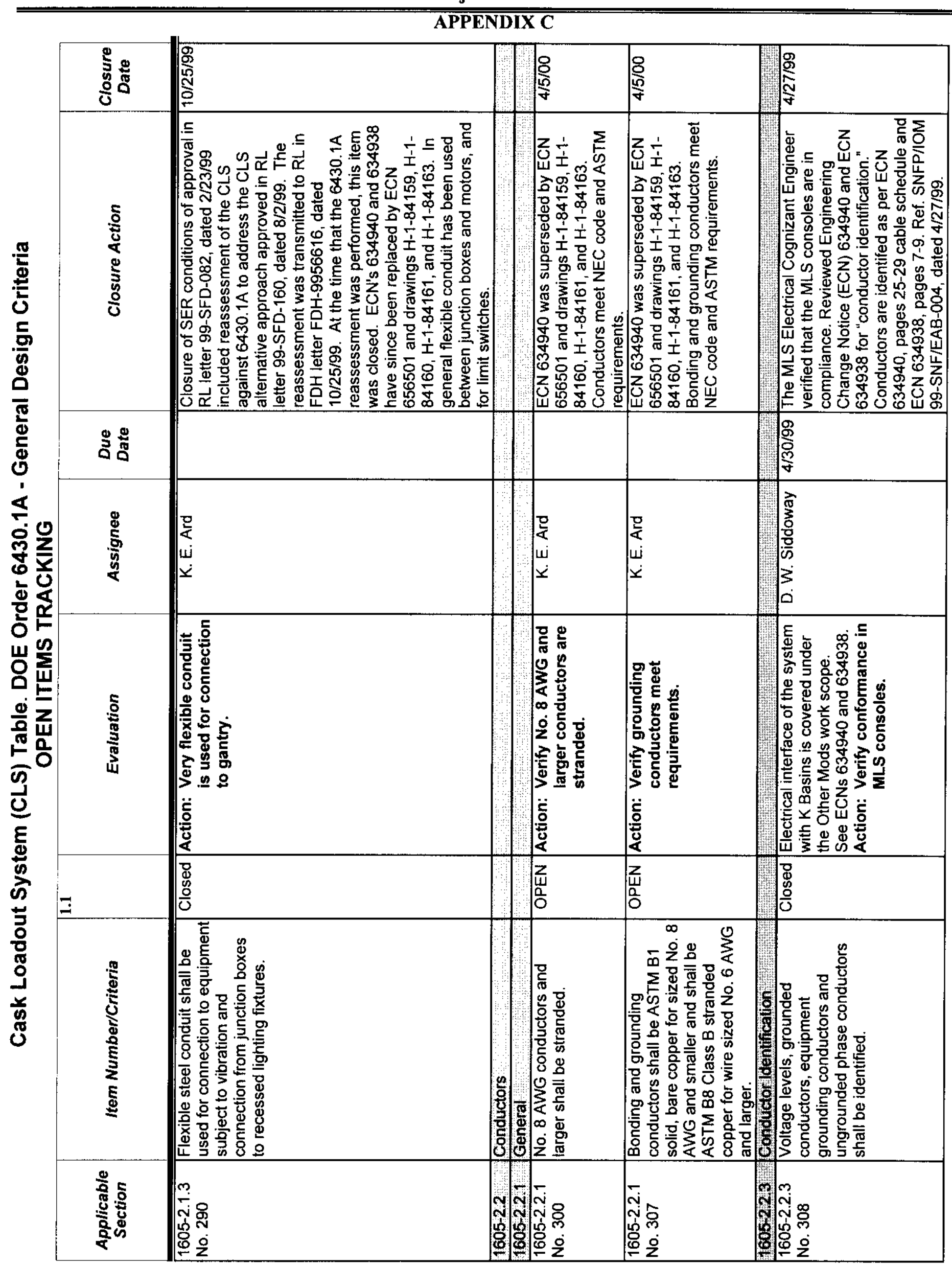


SSNF-4775, Rev. 2

Project A.5/A.6

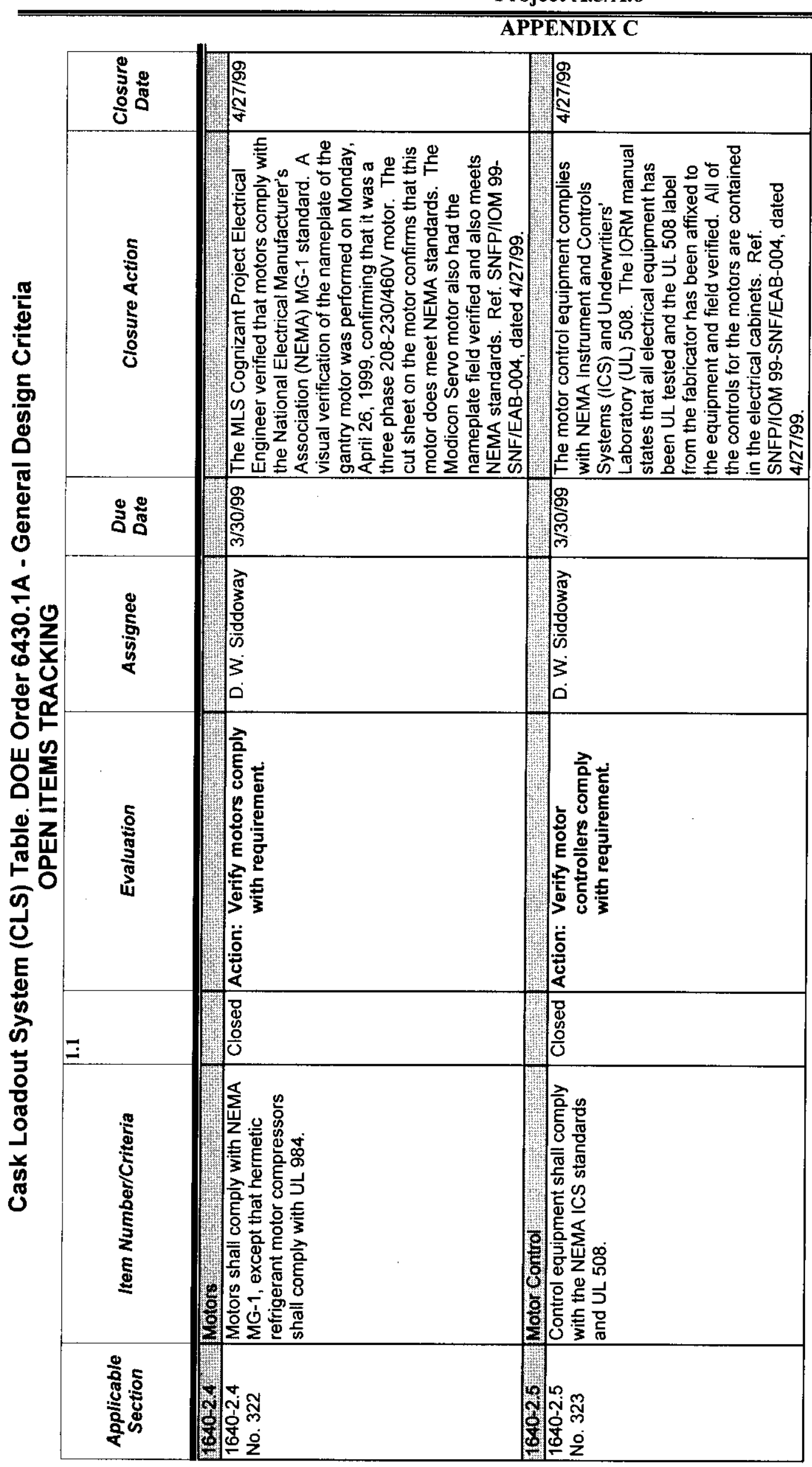

Page C-16 
SNF-4775, Rev. 2

Project A.5/A.6

APPENDIX D

CRANES - DOE ORDER 6430.1A - GENERAL DESIGN CRITERIA OPEN ITEMS TRACKING

OTHER MODS DOE ORDER 6430.1A - GENERAL DESIGN CRITERIA OPEN ITEMS TRACKING

CONSISTING OF 12 PAGES INCLUDING COVERSHEET

Page D-1 
SNF-4775, Rev. 2

Project A.5/A.6

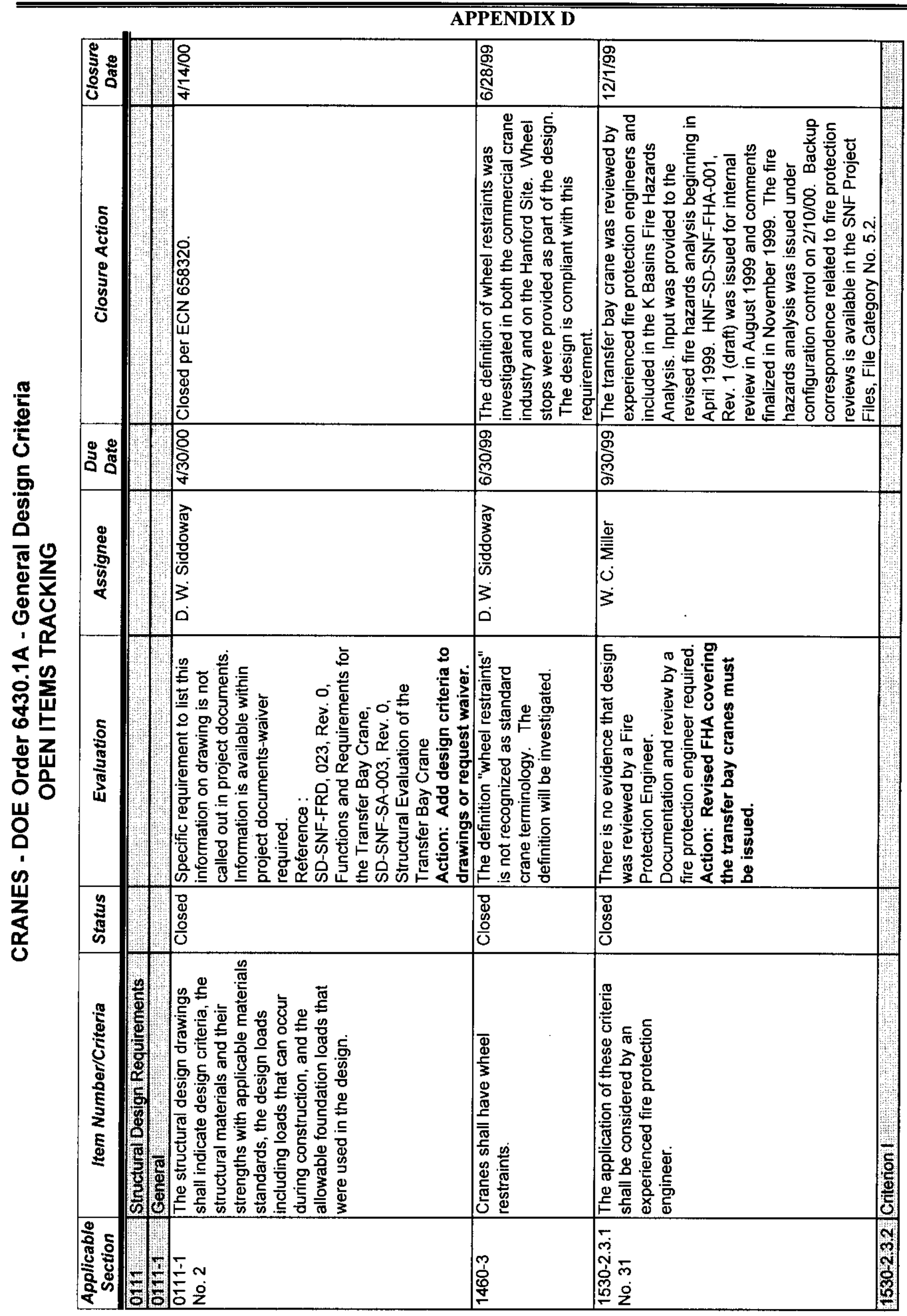

Page D-2 
SNF-4775, Rev. 2

Project A.5/A.6

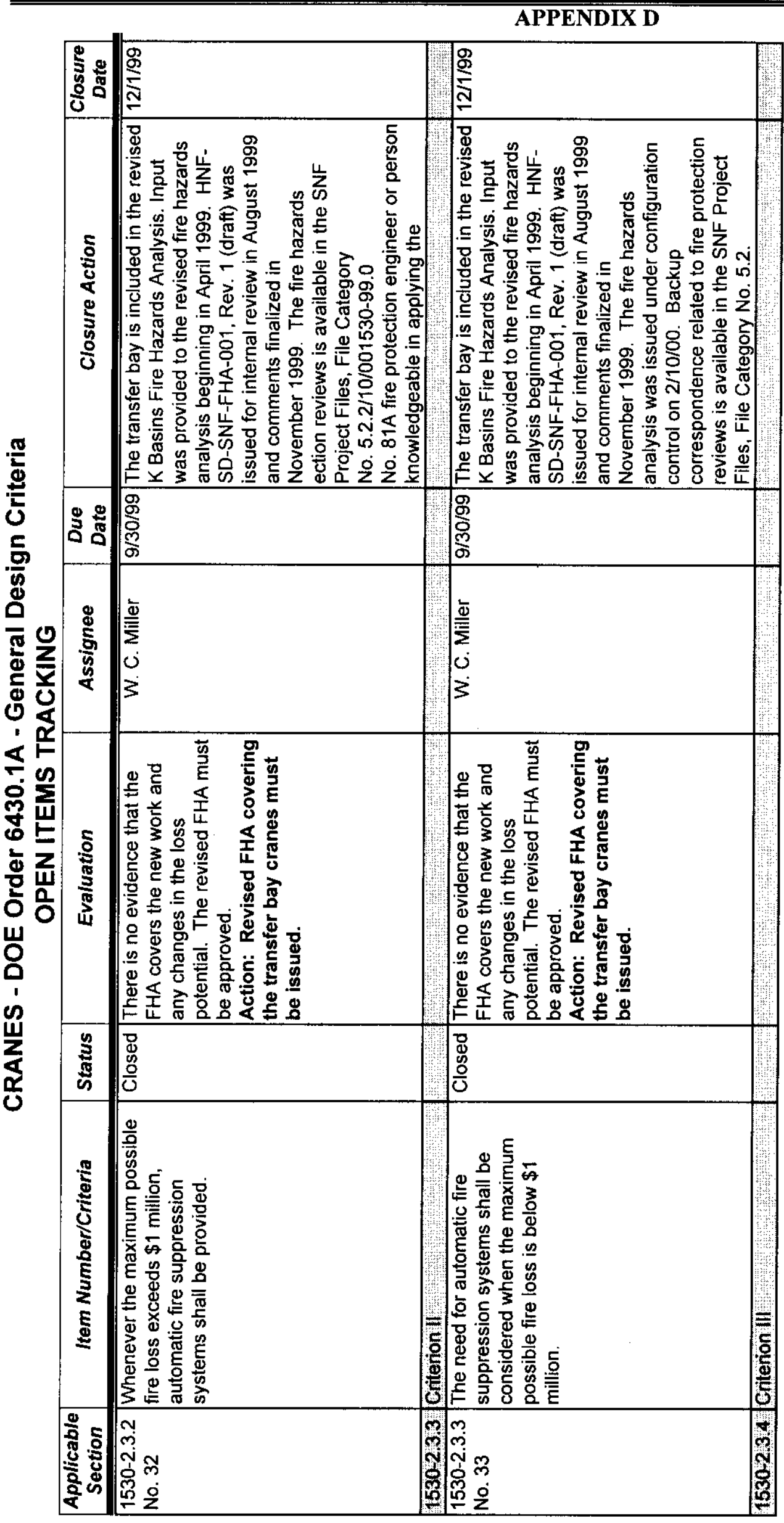

Page D-3 
SNF-4775, Rev. 2

Project A.5/A.6

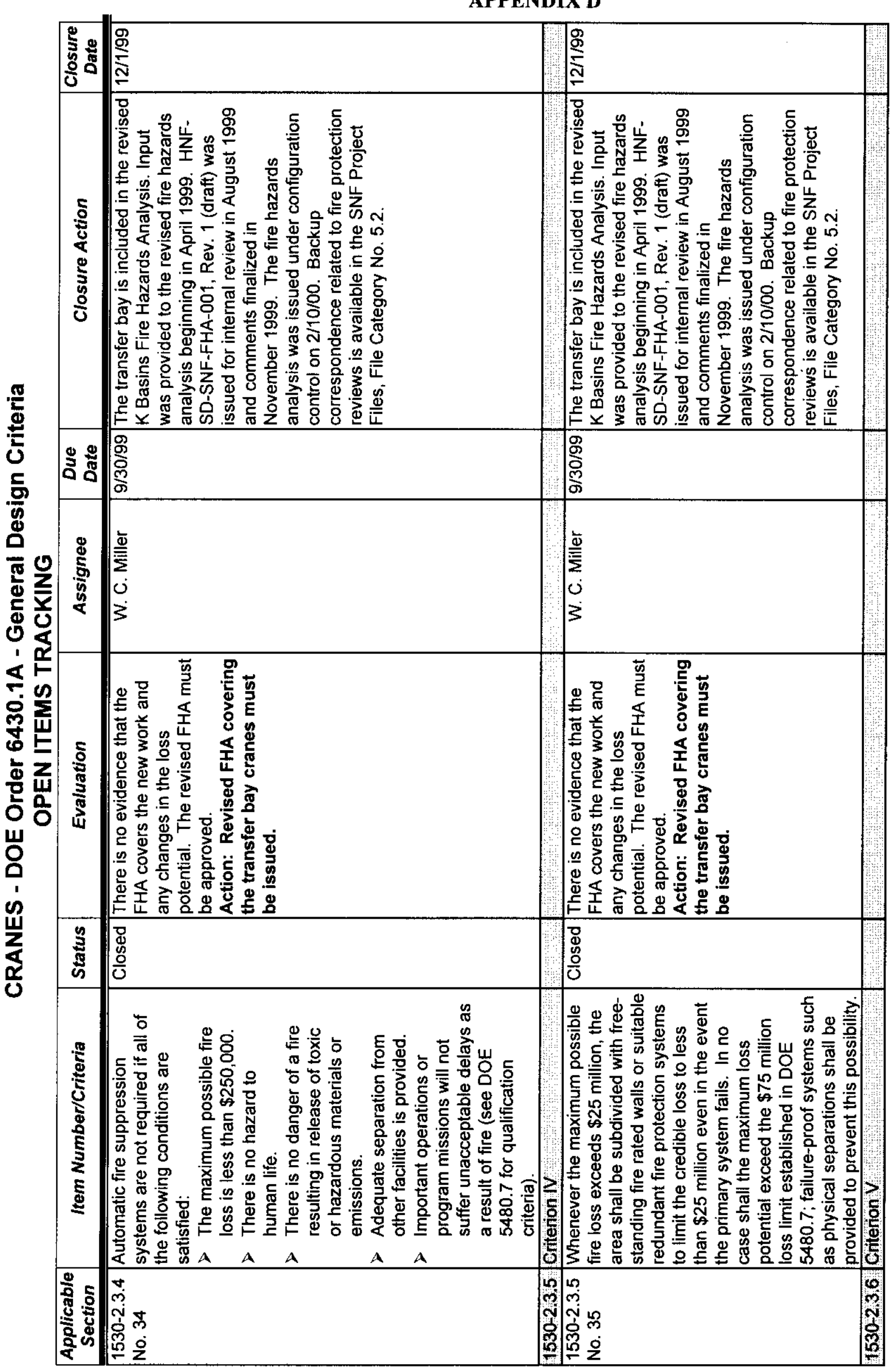

Page D-4 
SNF-4775, Rev. 2

Project A.5/A.6

APPENDIX D

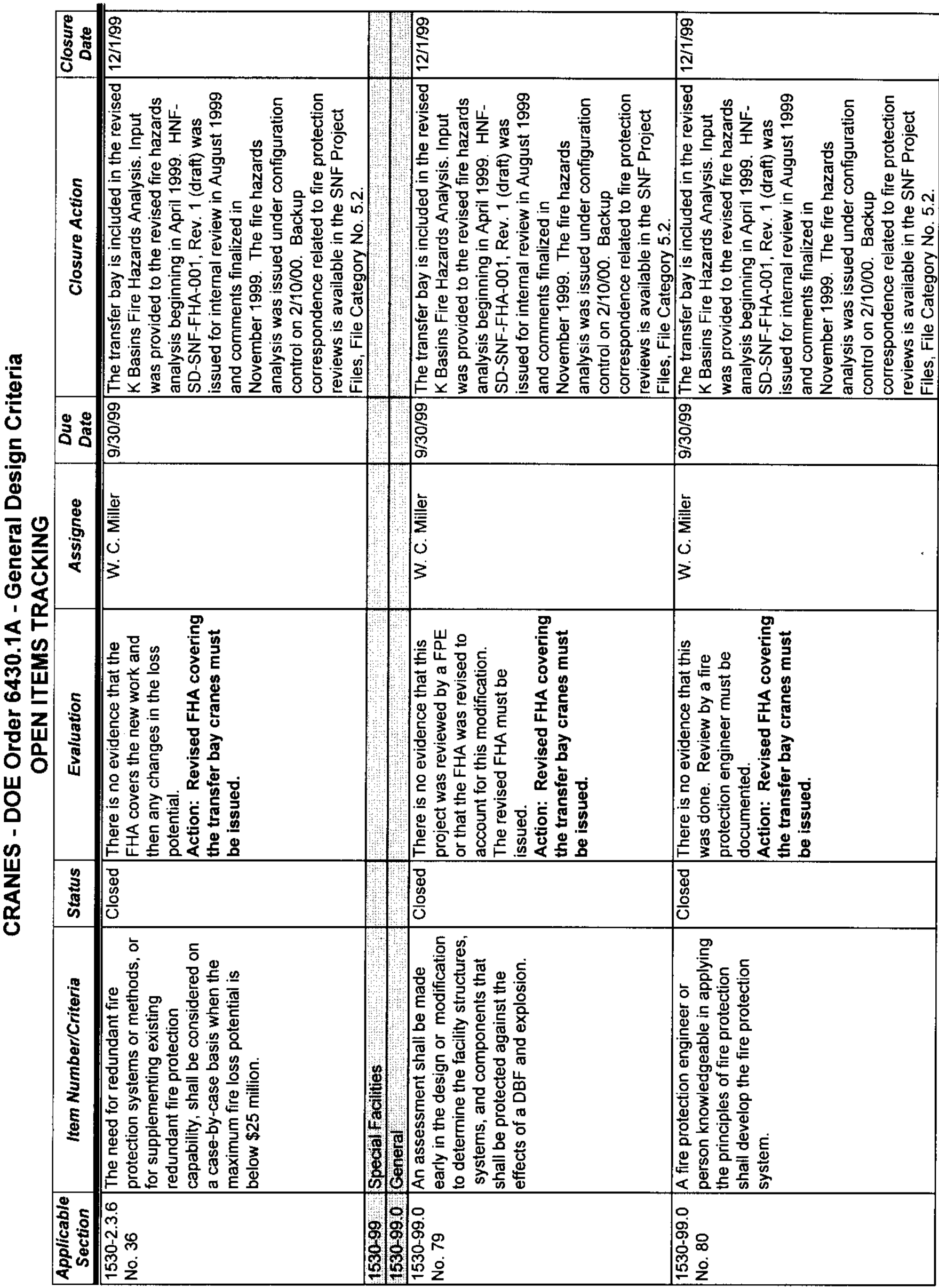

Page D-5 
SNF-4775, Rev. 2

Project A.5/A.6

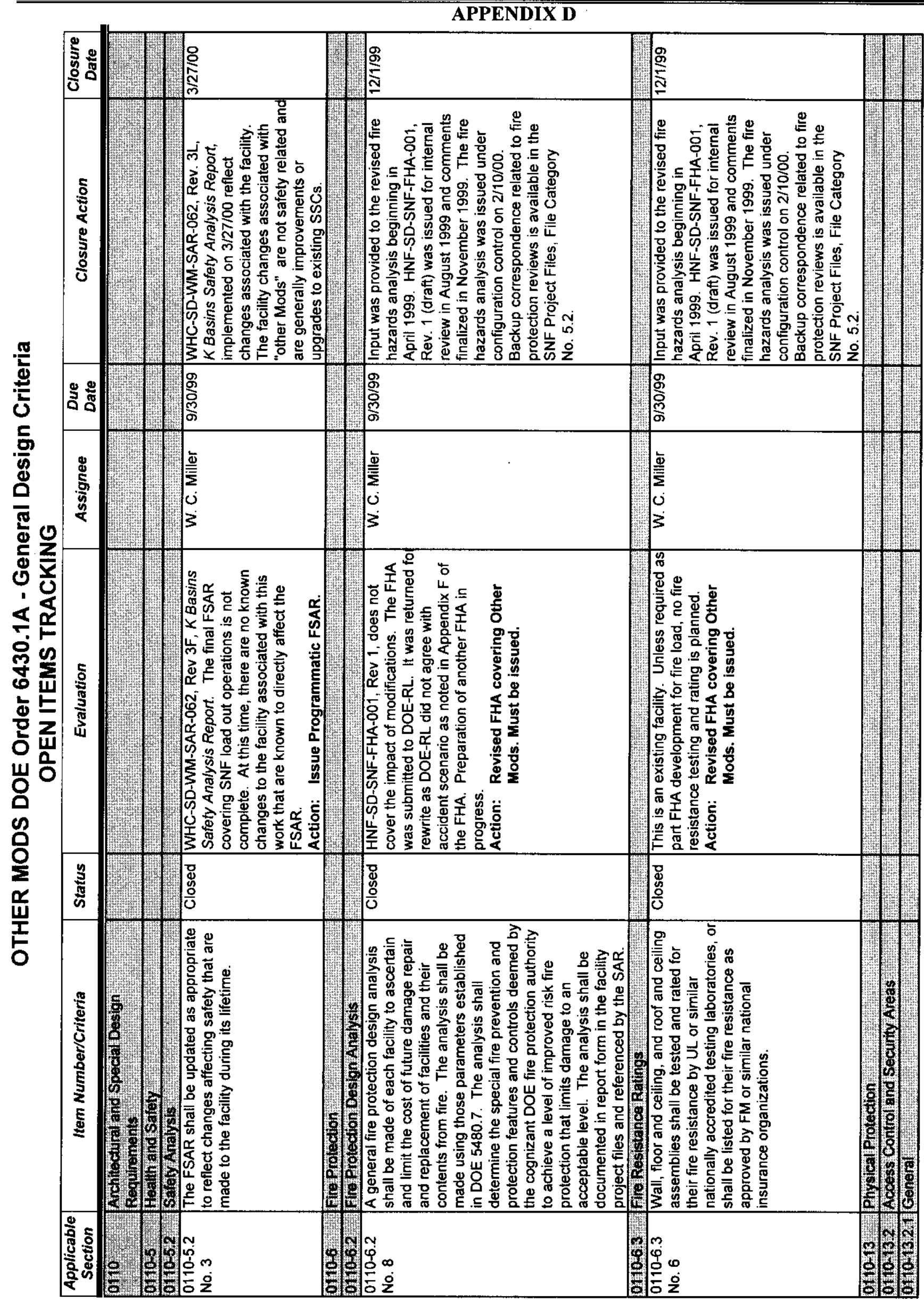

Page D-6 
SNF-4775, Rev. 2

Project A.5/A.6

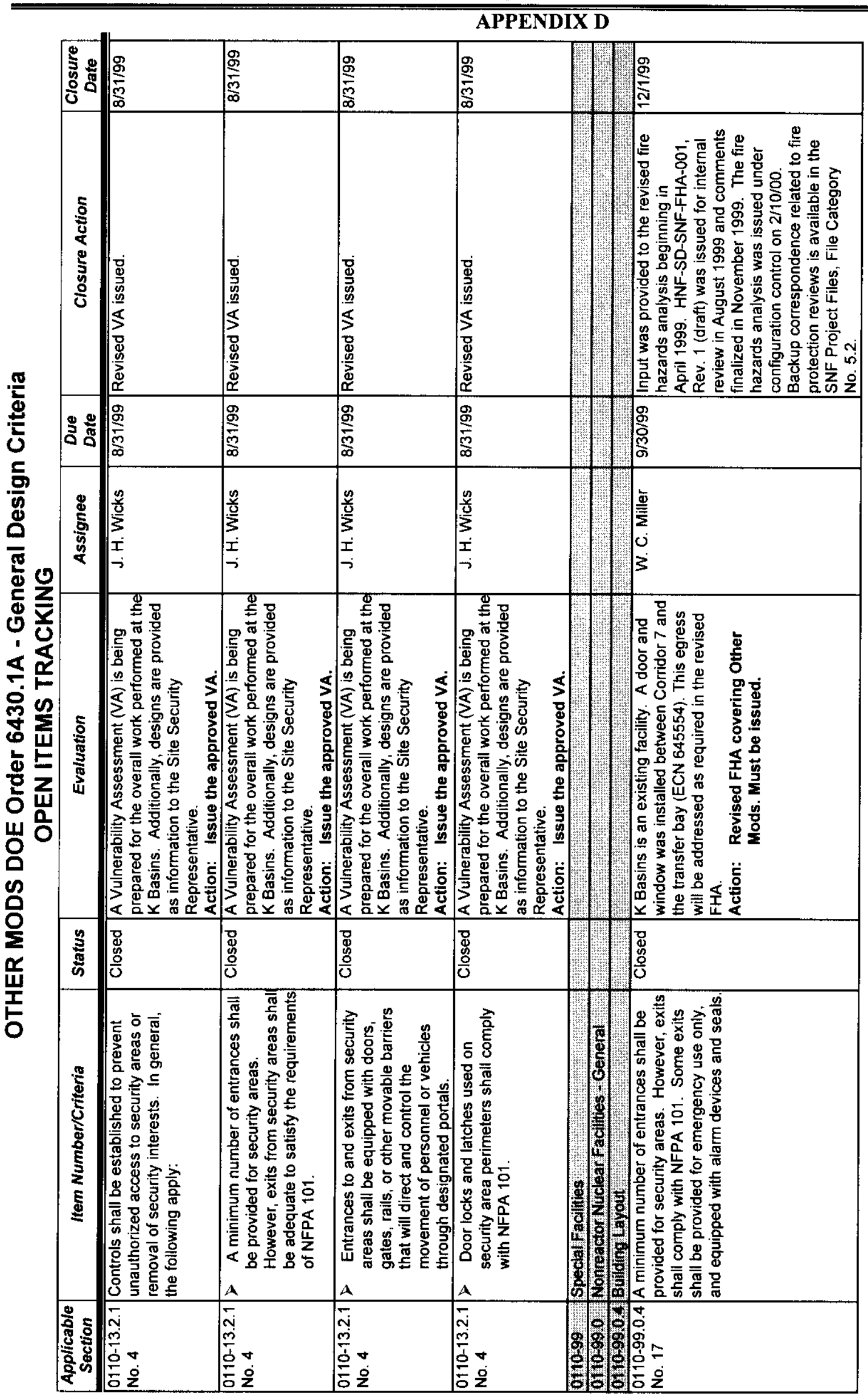

Page D-7 
SNF-4775, Rev. 2

Project A.5/A.6

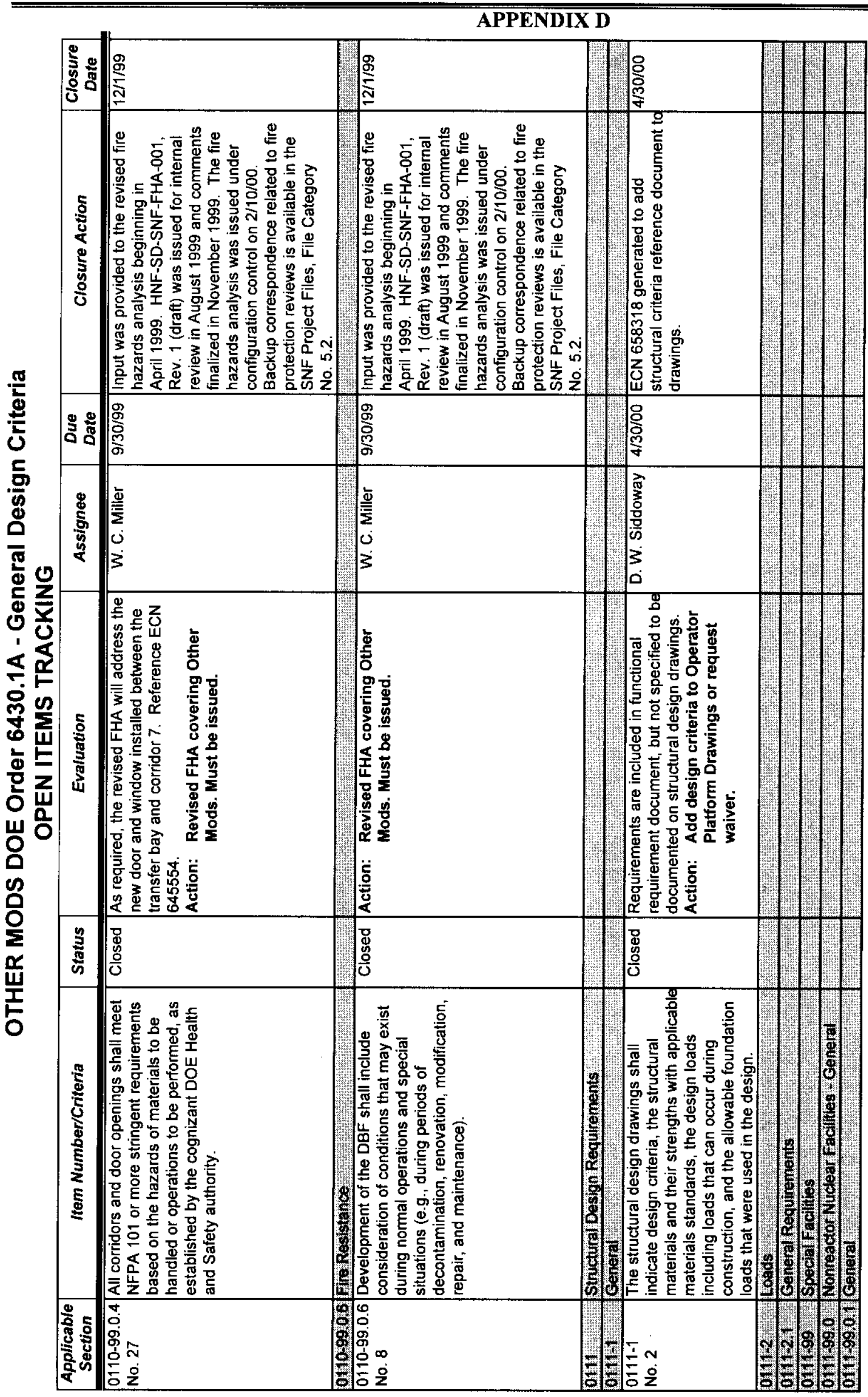


SNF-4775, Rev. 2

Project A.5/A.6

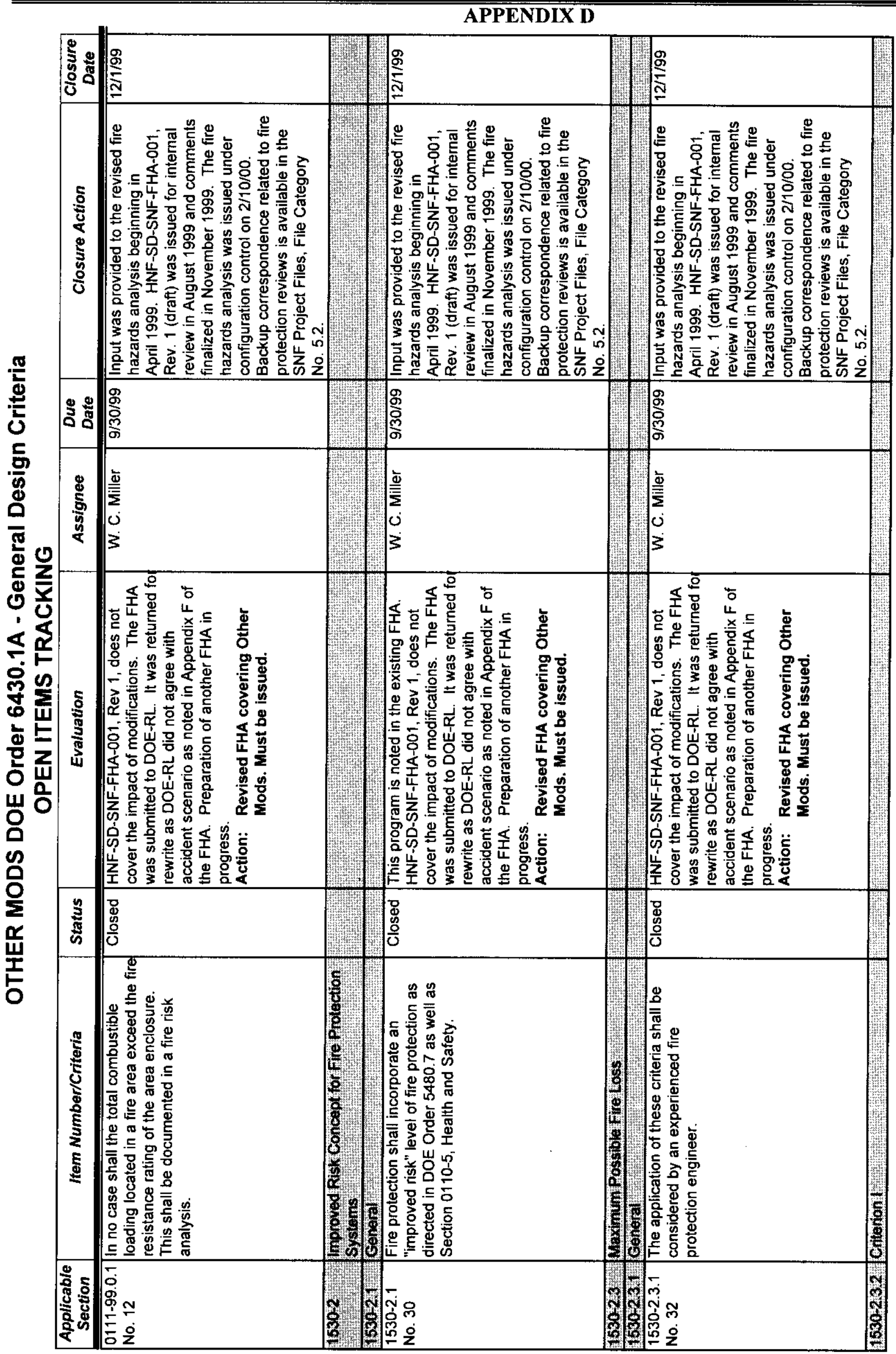

Page D-9 
SNF-4775, Rev. 2

Project A.5/A.6

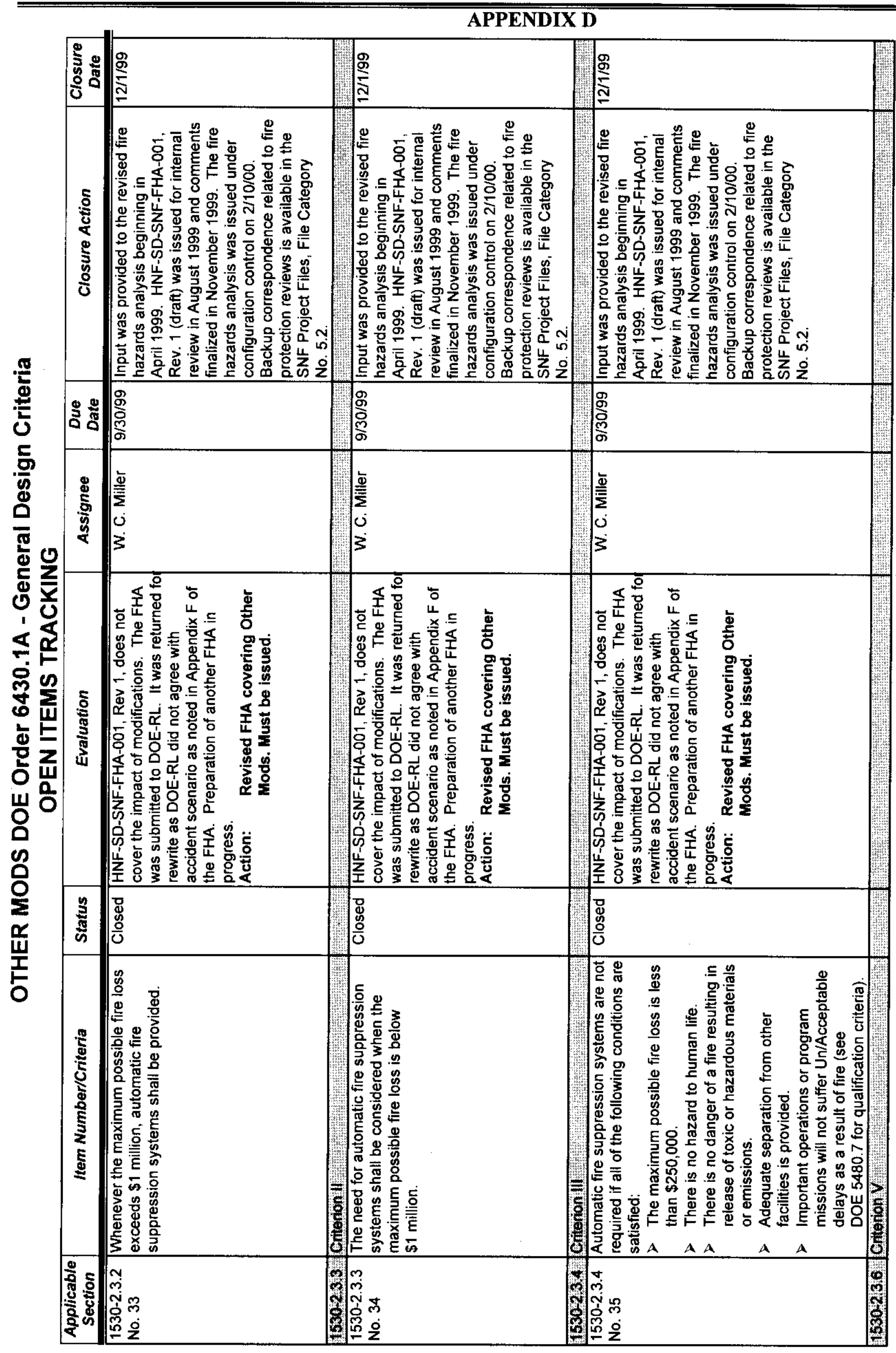

Page D-10 
SNF-4775, Rev. 2

Project A.5/A.6

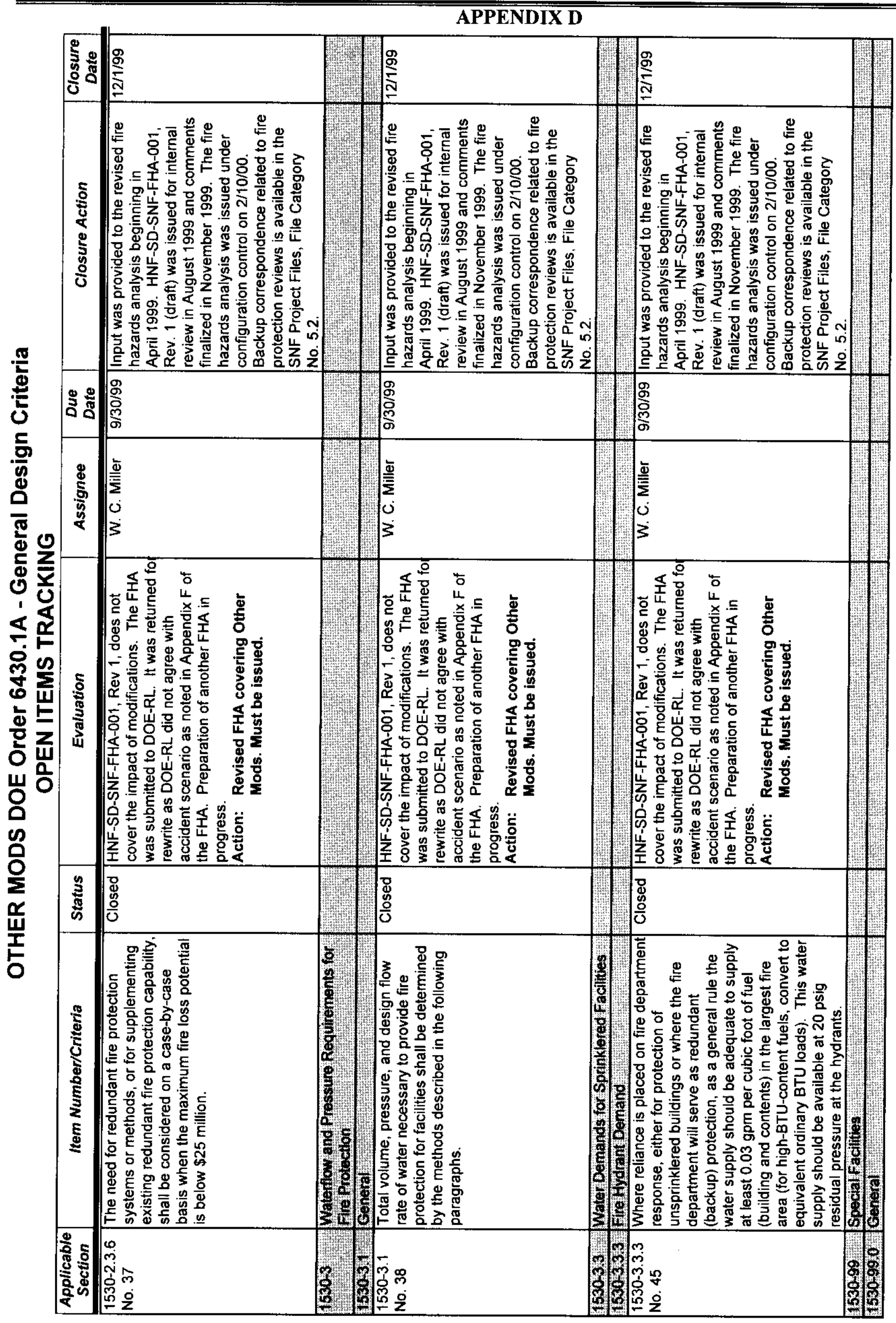

Page D-11 
SNF-4775, Rev, 2

Project A.5/A.6

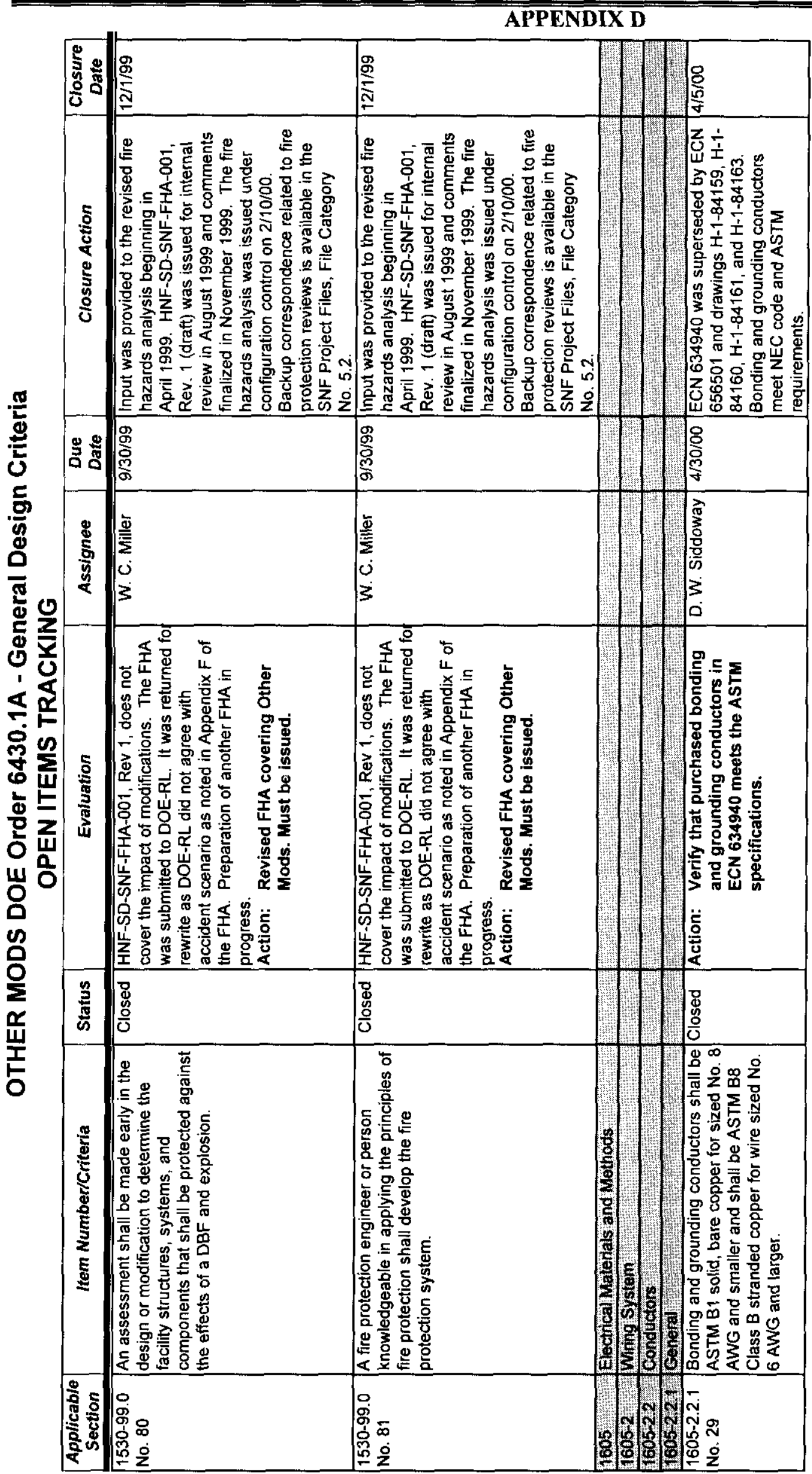

Page D-12 